\title{
New insights in the surgical treatment of chronic ischemic mitral regurgitation
}

Citation for published version (APA):

Gelsomino, S. (2009). New insights in the surgical treatment of chronic ischemic mitral regurgitation. [Doctoral Thesis, Maastricht University]. Datawyse / Universitaire Pers Maastricht. https://doi.org/10.26481/dis.20090424sg

Document status and date:

Published: 01/01/2009

DOI:

10.26481/dis.20090424sg

Document Version:

Publisher's PDF, also known as Version of record

\section{Please check the document version of this publication:}

- A submitted manuscript is the version of the article upon submission and before peer-review. There can be important differences between the submitted version and the official published version of record.

People interested in the research are advised to contact the author for the final version of the publication, or visit the DOI to the publisher's website.

- The final author version and the galley proof are versions of the publication after peer review.

- The final published version features the final layout of the paper including the volume, issue and page numbers.

Link to publication

\footnotetext{
General rights rights.

- You may freely distribute the URL identifying the publication in the public portal. please follow below link for the End User Agreement:

www.umlib.nl/taverne-license

Take down policy

If you believe that this document breaches copyright please contact us at:

repository@maastrichtuniversity.nl

providing details and we will investigate your claim.
}

Copyright and moral rights for the publications made accessible in the public portal are retained by the authors and/or other copyright owners and it is a condition of accessing publications that users recognise and abide by the legal requirements associated with these

- Users may download and print one copy of any publication from the public portal for the purpose of private study or research.

- You may not further distribute the material or use it for any profit-making activity or commercial gain

If the publication is distributed under the terms of Article $25 \mathrm{fa}$ of the Dutch Copyright Act, indicated by the "Taverne" license above, 
New Insights in the Surgical Treatment of Chronic Ischemic Mitral Regurgitation 
ISBN 9789052788258

(C) Sandro Gelsomino, Florence, Italy 2009

Prinred by Datawyse | Universitaire Pers Maastricht

Financial Support by Bio-Si International SRL, Florence, Italy and the University of Florence for the publication of this thesis is gratefully acknowledged.

Additional support was granted by St Jude Medical and Terumo. 


\title{
New Insights in the Surgical Treatment of Chronic Ischemic Mitral Regurgitation
}

\author{
DISSERTATION \\ to obtain the degree of Doctor at \\ the Maastricht University, \\ on the authority of the Rector Magnificus \\ Prof. dr. G.P.M.F. Mols, \\ in accordance with the decision of the Board of Deans, \\ to be defended in public \\ on Friday 24 April 2009, at 12.00 hours
}

by

Sandro Gelsomino

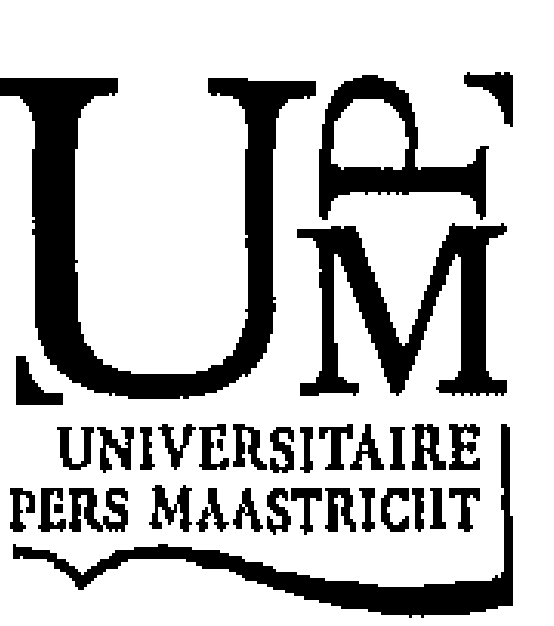


Supervisor

Prof. dr. JG Maessen

\section{Co-supervisors}

Dr. R Lorusso, Civic Hospital, Brescia, Italy

Dr. ir. FH van der Veen

\section{Assessment Committee}

Prof. dr. JH Zwaveling (chairman)

Prof. dr. AM Calafiore, Ospedale Ferrarotto, Catania, Italy Prof. dr. L Hofstra

Prof. dr. M Klautz, UMC Leiden 
"Two things awe me most, the starry sky above me and the moral law within me." Immanuel Kant, The Critique of Practical Reason

"Considerate la vostra semenza: fatti non foste a viver come bruti, ma per seguir virtute e canoscenza"

Dante Alighieri, Divina Commedia, Inferno canto XXVI, 116-120

"Consider ye the seed from which ye sprang; Ye were not made to live like unto brutes, But for pursuit of virtue and of knowledge."

Dante Alighieri, The Divine Comedy, Evil XXVI, 116-120,

Translation by Henry Wadsworth Longfellow

\section{To Cecilia}

"Shall I compare thee to a summer's day?

Thou art more lovely and more temperate: Rough winds do shake the darling buds of May, And summer's lease hath all too short a date: Sometime too hot the eye of heaven shines, And often is his gold complexion dimmed, And every fair from fair sometime declines,

By chance, or nature's changing course untrimmed:

But thy eternal summer shall not fade,

Nor lose possession of that fair thou ow'st, Nor shall death brag thou wander'st in his shade,

When in eternal lines to time thou grow'st, So long as men can breathe, or eyes can see, So long lives this, and this gives life to thee".

William Shakespeare. Sonnet XVIII

To my mother, my father, my grandmother Carmela my sister Gabriella, my aunt Maria,

my nephew Gianmarco, my nieces Federica and Valentina, my brother-in-law Orlando and my devoted friend Francesco.

"When you turn the look to your life, the greatest joys are those of the family."

$$
\text { J. Brothers }
$$




\section{TABLE OF CONTENTS}

Preface

Acknowledgements

Abbreviations and acronyms

Chapter I

Introduction

Chapter 2

Five-year echocardiographic results of combined undersized mitral ring annuloplasty and coronary artery bypass grafting for chronic ischemic mitral regurgitation

\section{Chapter 3}

Left ventricular reverse remodeling after undersized mitral ring annuloplasty in patients with ischemic regurgitation

\section{Chapter 4}

Insights on left ventricular and valvular mechanisms of recurrent ischemic mitral regurgitation after restrictive annuloplasty and coronary artery bypass grafting

Chapter 5

Impact of anterior leaflet tethering on recurrence of ischemic mitral regurgitation after restrictive annuloplasty

\section{Chapter 6}

Left ventricular diastolic function after restrictive mitral ring annuloplasty in chronic ischemic mitral regurgitation and its predictive value on outcome and recurrence of regurgitation

\section{Chapter 7}

Prognostic value of doppler-derived mitral deceleration time on left ventricular reverse remodeling after undersized mitral annuloplasty

\section{Chapter 8}

Conclusions

\section{Chapter 9}

Summary

Riassunto

Samenvatting

Publications

Curriculum vitae 


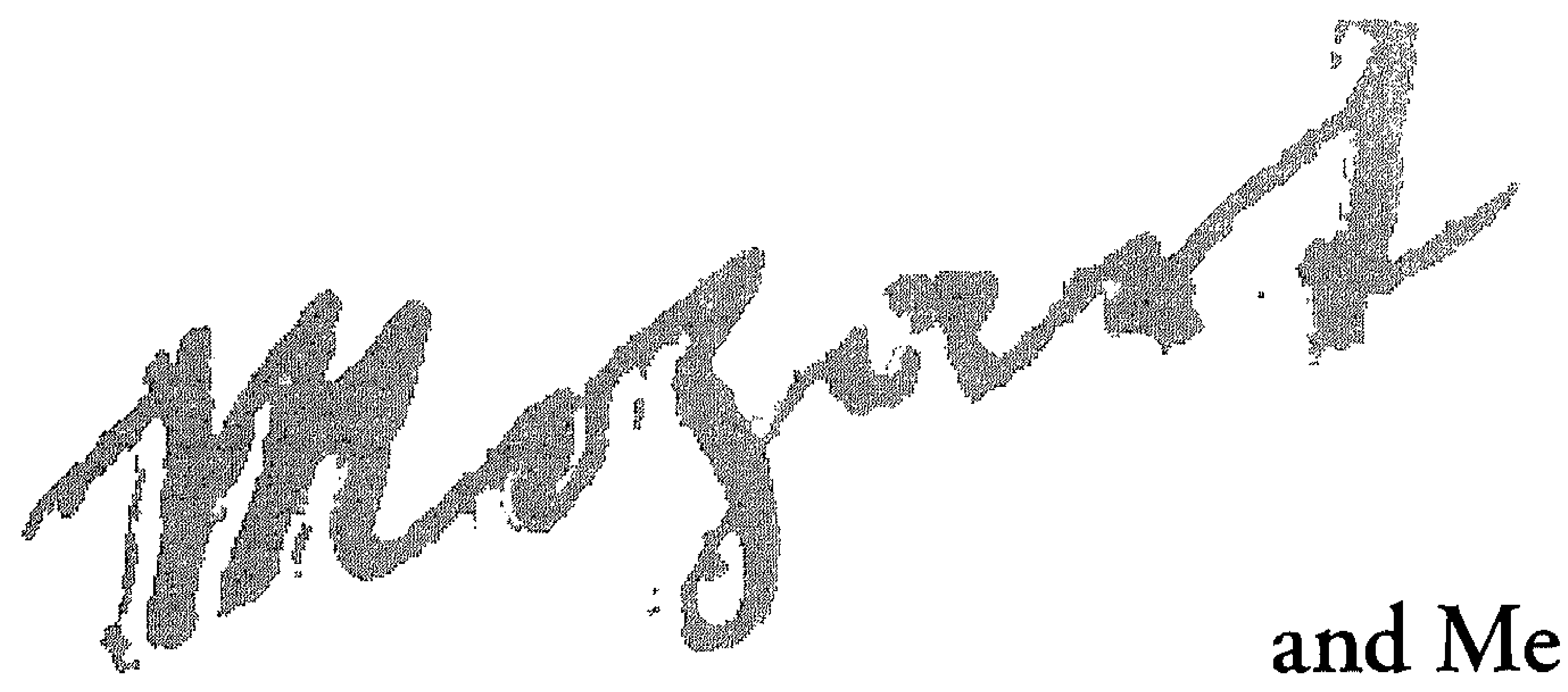

Music was probably born of the natural rhythms of life. Since ancient the time of Greece, music has been able to excite people, or calm people. Orpheus, the son of Calliope, on a voyage with Jason and the Argonauts, played lively melodies on his lyre to energize fatigued oarsmen, and shooting tunes them when they quarreled.

It should not come as a surprise when people who dedicate themselves to life science release their creative energy in music. Music has always been an interesting domain for the application of new scientific discoveries inviting composers to combine artistic creativity with scientific methods.

Indeed both music and medicine are strict disciplines, even though one is considered an art and one a science. Both demand practitioners who learn complex skills, practice them exhaustively, then impose a set of rules on what is naturally messy-a disease, an injury, a series of unrelated sounds. And both require enormous dedication, so much that it would seem impossible for one person to do both. But some scientists manage. They realize medicine and music are not mutually exclusive.

Not only can the two disciplines coexist; given the right conditions, they can coalesce.

I was raised in a family where Mozart was never played.

My family was unusual in the respect that both my parents enjoyed classical music, but neither were Mozart fans. In fact, my dad never played Mozart because he said his music was "too happy".

I first heard "Don Giovanni K 527" as a 10-year-old boy and I was struck immediately by the power of Mozart's music. Then I heard "Madamina il catalogo è questo", "La ci darem la mano", "Dalla sua pace", "Il mio tesoro intanto"again, and then again, and I heard more and more in it, until it became the soundtrack of my life. Since then Mozart's Fantasia in D Minor K.397, his clarinet concerto in A major K. 622, the piano Concerto No 20 in D Minor K466, have inspired and 
encouraged me in every day life. His music reminded me of possibility, hope and belief. I turned to his melodies when I felt down, in need of courage or to express my feelings. The magnificence of his music helped me turn sadness into hope or fear into courage. His eternal melodies helped me to believe in love, to know I was not alone, to laugh, to cry...

There is something magical in his music because, after more than 35 years, in re-listening to The Marriage of Figaro K492", "Die Zauberflöte K620", "Die Entfuhrung aus dem Serail K.384, ", I am affected again and again and the more I listen to Mozart's music, the more I love it. His music still touches me profoundly, because it is so luminous and yet at the same time so deep." His music is by no means just entertainment; it contains the whole tragedy of human existence", the Holy Father Benedict XVI recently said.

In a normal day at the hospital, the Mozart's music fill the operating theatre: it relaxes me, and focuses my attention. I think that any music could be good for routine operation but only Mozart for trickier ones. All my research was deeply inspired by Mozart's music and I couldn't spend many, many hours in the Experimental Surgery laboratory without listening to "un'aura amorosa", "la ci darem la mano", "che soave zeffiretto". His music carries an important component of human thought, namely formal abstraction, which can be very inspiring for medical research.

Mozart' music is universal. He speaks to almost everybody and he never pushes us. If all you want from one of his compositions are some good tunes and seraphic harmonies, he will surely provide them. If you want much, much more, Mozart, in virtually all of his mature pieces, will provide that, too. Bach and Beethoven tell us exactly what they want to tell us, while Mozart lets us find what we want in him, on our own levels of need and understanding.

In 1906, the pianist and composer Ferruccio Busoni was asked to write a series of aphorisms to celebrate the 150th anniversary of Mozart's birth and 100 years later most of us would echo what he had to say. "... Up to now he is the most complete manifestation of musical gifts... He disposes of light and shadow, but his light does not pain and his darkness still shows clear outlines... He is young as a boy and wise as an old man, never old-fashioned and never modern, carried to the grave and always alive.

... His smile, which was so buman, still shines on us transfigured." 


\section{ACKNOWLEDGEMENTS}

I would like to express my endless gratitude to Roberto Lorusso who brought me out of the darkness.

I would like to extend my heartfelt thanks to Professor Gian Franco Gensini for his invaluable support to my research.

Special appreciation should be devoted to Erik van der Veen and Jos Maessen who strongly supported me for the Doctorate. Without them and Roberto I would never have been here.

A special thank has to be addressed to my friends Giuseppe De Cicco who made most of the illustrations of this thesis, and Mario Romano for his friendly help.

Thank you to my brother-in-law Orlando for your help in statistical analysis.

Finally, I would like to reserve a special thank to all colleagues cooperating in my research. 


\begin{tabular}{ll} 
IMR & Ischemic Mitral Regurgitation \\
CIMR & Chronic Ischemic Mitral Regurgitation \\
UMRA & Undersized Mitral Ring Annuloplasty \\
MI & Myocardial Infarction \\
MV & Mitral Valve \\
MR & Mitral Regurgitation \\
IMLC & Incomplete Mitral Leaflet Closure \\
CAD & Coronary Artery Disease \\
CABG & Coronary Artery Bypass Grafting \\
PM(s) & Papillary Muscle(s) \\
PPM & Posterior Papillary Muscle \\
APM & Anterior Papillary Muscle \\
LV & Left Ventricle \\
LVRR & Left Ventricular Reverse Remodeling \\
ERO(A) & Effective Regurgitant Orifice (Area) \\
RF & Regurgitant Fraction \\
RV & Regurgitant Volume \\
PISA & Proximal Isovelocity Surface Area \\
NYHA & New York Heart Association \\
CCS & Canadian Cardiovascular Society \\
TEE & Transesophageal Echocardiography \\
TTE & Transthoracic Echocardiography \\
EDD & (Left ventricular) End Diastolic diameter \\
ESD & (Left ventricular) End Systolic diameter \\
LA & Left Atrium (mm) \\
ESVI & (Left ventricular) End Systolic Volume Index \\
EDVI & (Left ventricular) End Diastolic Volume Index \\
LVEF & Left Ventricular Ejection Fraction \\
MPI & Myocardial Performance Index \\
SPI & Sphericity Index \\
WMSI & Wall Motion Score Index; \\
AML & Anterior Mitral Leflet \\
PML & Posterior Mitral Leflet \\
TA & Tenting Area \\
CH & Coaptation Height \\
CL & Coaptation Length \\
MAs & (Systolic) Mitral Annular Area \\
Mad & (Diastolic) Mitral Annular Area \\
MAp & Trans Mitral Diastolic Gradient \\
$\alpha^{\prime}$ & Anterior Mitral Leaflet Tethering Angle \\
$\beta^{\prime}$ & Posterior Mitral Leaflet Tethering Angle \\
$\beta_{\text {Ex }}$ & Anterior Mitral Leaflet Excursion Angle \\
& Posterior Mitral Leaflet Excursion Angle \\
& Bending Angle \\
\hline Interquartile Range \\
\end{tabular}




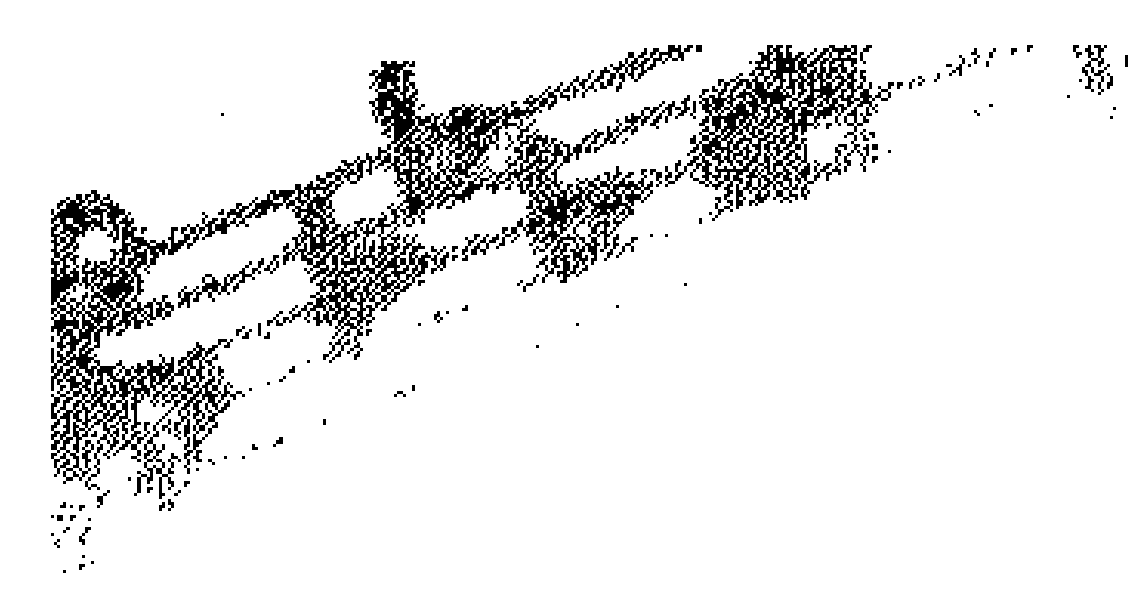

1

Introduction

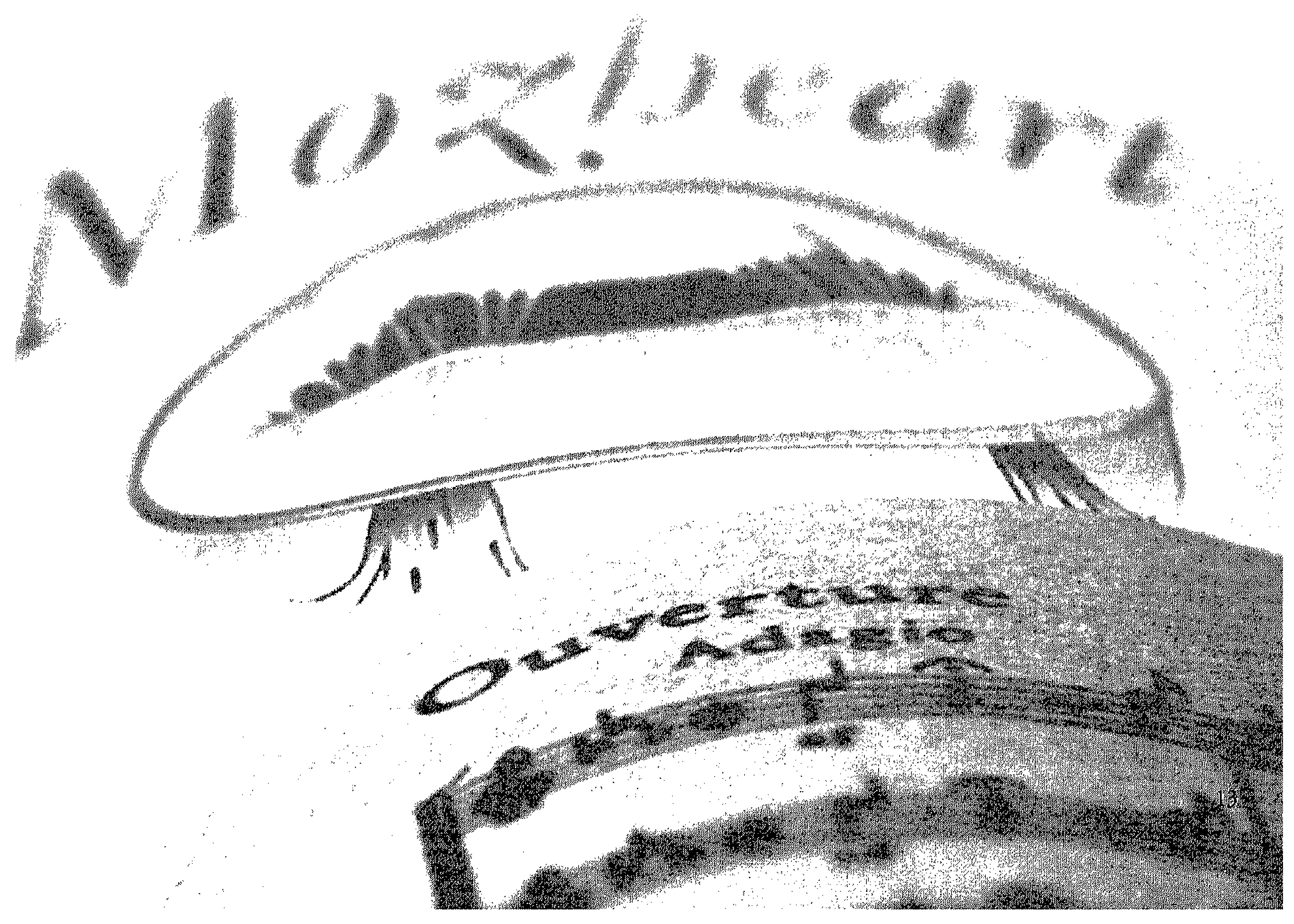




\section{INTRODUCTION}

Ischemic mitral regurgitation is a relatively common manifestation of coronary artery disease and occurs after acute myocardial infarction (MI) or as a chronic condition, and less commonly as regional episodic ischemia. Chronic Ischemic mitral regurgitation (CIMR) is a functional mitral regurgitation (MR), as opposed to organic MR, because of the normal appearance of the mitral valve (MV) leaflets. CIMR is associated with a markedly worse prognosis after myocardial infarction (MI) and coronary revascularization ${ }^{1-5}$, and is a common cause of post-MI congestive heart failure $e^{6,7}$. CIMR is present in $10 \%$ to $20 \%$ of patients with coronary artery disease $(\mathrm{CAD})^{8-10}$.

\section{Definition}

Ischemic mitral regurgitation should be distinguished from mitral regurgitation due to structural leaflet disease (such as rheumatic or mitral valve prolapse) and fortuitously associated with coronary artery disease. Conversely, mitral regurgitation of ischemic etiology can be the consequence of papillary muscle rupture, which represents a severe, albeit rare, complication of acute MI that requires emergency intervention ${ }^{11-13}$.

Chronic IMR should be defined as mitral regurgitation occurring more than 1 week after MI with (1) one or more left ventricular segmental wall motion abnormalities; (2) significant coronary disease in the territory supplying the wall motion abnormality; and (3) structurally normal MV leaflets and chordae tendineae. The third criterion is particularly important as it excludes patients with organic MR and associated CAD.

\section{Historical NOTES}

Burch, De Pasquale, and Phillips ${ }^{13,14}$ initially ascribed ischemic MR to papillary muscle (PM) dysfunction. Normally, as the left ventricle (LV) shortens, PM contraction maintains the distance between the PM tips and mitral annulus to prevent prolapse. PM damage could then produce prolapse, consistent with its frequently late-systolic murmur. Experimental damage to the PMs themselves, however, fails to produce MR acutely without damage to the underlying myocardial wall as well ${ }^{15}$. Burch et a $1^{13,14}$ alternatively postulated restricted leaflet motion. The PMs, normally parallel to the LV long axis and perpendicular to the leaflets, efficiently balance forces generated by ventricular pressure on the leaflet surface. Ischemia or heart failure causes the myocardial segments underlying the PMs to bulge posterior and outward, displacing the PMs, so that they pull the leaflets non perpendicularly, away from their normal coaptation. The distance between the PM tips and the annulus 
also increases, drawing the leaflets into the ventricle and restricting their motion toward closure, as proposed by Paul Dudley White, Levy and Edwards, Silverman and $\mathrm{Hurst}^{16}$, and Perloff and Roberts ${ }^{17}$. Two-dimensional echocardiography first directly demonstrated apical restricted leaflet motion, termed "incomplete mitral leaflet closure" (IMLC) ${ }^{18,19}$. Godley, Weyman, et a ${ }^{19}$ associated IMLC with inferior dyskinesis, postulating increased leaflet tethering. Based on myocardial injection studies, Alain Carpentier has succinctly renamed PM dysfunction "PM wall dysfunction", namely, tethering caused by displacement of the leaflet attachments. Kaul et a $1^{2 n}$ elegantly confirmed that reducing PM perfusion produced neither prolapse nor MR. In contrast, global hypoperfusion with LV dilatation, despite continued PM perfusion and thickening, caused MR with IMLC in direct correlation with LV dysfunction ${ }^{20}$. In those studies, however, akinesia of the PMs and their underlying wall also failed to produce MR acutely. This led to the postulate that MR results from global LV dysfunction, decreasing the leaflet closing force, ${ }^{21}$ as opposed to PM wall tethering. Conceivably, however, the acute PM wall displacement in that study was insufficient to produce MR, and as the authors acknowledged, LV dilatation paralleled global dysfunction, precluding separation of dysfunction from dilatation as primary cause of MR. This distinction has practical implications: LV contractile dysfunction would demand inotropic therapy, revascularization, or transplantation, whereas tethering might respond to modifying LV wall or PM geometry. New models were therefore required to dissect the effects of dysfunction and dilatation. He et al ${ }^{22}$ reproduced MR in excised mitral valves by displacing the PMs apically, posteriorly, and outward, as in patients. In vivo, Otsuji et $\mathrm{al}^{23}$ created global LV dysfunction pharmacologically but limited LV expansion through pericardial restraint and decreased preload. Contractile dysfunction (ejection fraction $<20 \%$ ) without dilatation produced only trace MR; LV dilatation was a prerequisite for IMLC and $M^{23}$. To quantify tethering geometry, these authors used $3 \mathrm{D}$ echocardiographic data to standardize the tethering length from the PM tips to the anterior mitral annulus. In global dysfunction, Otsuji et $\mathrm{a}^{23}$ found that tethering length was the only independent predictor of MR, not LV ejection fraction or $\mathrm{dP} / \mathrm{dt}$. MR also correlated with $\mathrm{LV}$ sphericity, consistent with the key observation of Kono, Sabbah et a ${ }^{24-26}$ increased sphericity that could potentially displace the PMs posterolaterally, as verified by $3 \mathrm{D}$ echocardiography ${ }^{23}$. The centrality of geometric change was confirmed in acute segmental inferior ischemia, studied with and without external constraint ${ }^{27}$, and in the chronic sheep model of Gorman and Edmunds, in which MR, initially absent, evolves in parallel with LV remodeling and PM displacement ${ }^{28-30}$. In patients, Yiu, Enriquez-Sarano et al ${ }^{31}$ strongly related PM tethering, leaflet tenting, and MR, which is frequently due to localized remodeling of the posterior PM-bearing walls, without the global remodeling required for $\mathrm{MR}$ with anterior infarctions ${ }^{10}$. By 3D echocardiography, the medial mitral valve is most tented in ischemic cardiomyopathy, because the inferomedial PM is most displaced ${ }^{32}$; this asymmetry might 
augment MR through asymmetrical coaptation between posterior leaflet scallops ${ }^{33}$. Although the annulus is dilated in global dysfunction ${ }^{34}$, isolated annular dilatation without increased tethering length in atrial fibrillation is not associated with important $\mathrm{MR}^{35}$. Finally, Otsuji and colleagues ${ }^{36}$ recognized restricted diastolic as well as systolic leaflet excursion, an echocardiographic clue that tethering is increased. The simple story, however, is often incomplete. First, although PM damage does not produce MR acutely, MR does occur in the chronic phase of ethanol injection, with PM scarring and retraction ${ }^{15}$. Second, occasional patients with MI actually do have prolapse with PM elongation ${ }^{37}$. These observations, rather than disproving tethering, actually confirm that tethering distance is the final common pathway determining the level of leaflet coaptation. The change in tethering distance is the resultant of two vectors: Displacement of the PM-bearing wall is modulated by changes in PM length. As Frater et $\mathrm{a}^{38}$ have shown, MR increases biphasically with too long or too short a tethering length. We can therefore understand how PM contractile dysfunction can paradoxically diminish MR. Messas et a ${ }^{39}$ created a limited inferobasal MI with wall bulging and MR; making the adjacent PM acutely ischemic caused it to elongate in response to ventricular forces and reduced tethering distance and MR. Preserved PM shortening increases tethering and $\mathrm{MR}^{40}$, which confirms the central role of tethering length and the concept of PM-ventricular wall complex expressed by Komeda, Miller et $\mathrm{al}^{41}$. Tethering is one facet of a unifying principle of mitral valve function: that leaflet motion is determined by the $3 \mathrm{D}$ geometry of the leaflets and their attachments, relative to the surrounding flow field. Another example is the relation between systolic anterior mitral valve motion and anterior PM displacement combined with leaflet elongation, the converse of posterior PM displacement and leaflet restriction in ischemic MR.

The dichotomy between tethering versus closing forces is also a simplification: both are involved. The equilibrium position of the mitral leaflets is determined by the balance of forces acting on them, including annular and PM tethering forces and LV-generated closing forces. Normally, little force is required to close the thin leaflets, so MR is not produced by global dysfunction without tethering ${ }^{23}$. However, once tethering is increased, leaflet closure is further. This balance of competing forces creates a unique dynamic pattern, recognized by Schwammenthal et $\mathrm{a}^{42}$, with flow and orifice area greatest in early and late systole and often paradoxically decreased in mid systole, when peak LV pressure maximizes leaflet closure. Tethering sets the stage for MR; transmitral pressure modulates its variation throughout the cardiac cycle $^{22,43}$. We therefore understand the early systolic leaflet "loitering" described by Glasson, Miller et $\mathrm{a}^{44}$ and late systolic MR and murmurs in patients with inferior MIs, in whom tethering increases as the inferior wall is "left behind" the contracting ventricle ${ }^{45}$. 
The mitral valve has a complex anatomy contributing actively to the process of systolic valvular closing. This mechanism consists of a combination of interactions of the ventricle, annulus, and papillary muscles. Therefore, any alterations in one or more of these structures may contribute to the regurgitation. Normal MV function requires coordinated operation of all components including the annulus, leaflets, chordae tendineae, and papillary muscles (PMs). The annulus is a saddle like structure whose main function is to act as a fulcrum for the mitral leaflets and to decrease the size of the mitral orifice (by $10 \%$ to $20 \%$ ) during late diastole and systole ${ }^{46-48}$. A dilated mitral annulus is often reported as an important etiologic factor in CIMR ${ }^{49}$, but isolated annular dilation does not cause significant $M^{50}$. The second component of the MV apparatus are the leaflets. The leaflets are morphologically normal in CIMR, but there is tethering and retraction of the leaflet bodies. As stated before, it is important to exclude patients with organic MV leaflet pathology and associated CAD from CIMR studies, as these patients have a much better prognosis. Chordae tendineae are the third component of the MV apparatus, connecting the PMs to the leaflets. Primary chordae attach to the free edge of the leaflets and prevent prolapse during systole. Secondary chords attach to the belly of the leaflet and are thicker than primary chords.

Apical displacement of the PMs leads to tethering of the secondary chords and decreased leaflet coaptation. The final component of the MV apparatus is the PMs, named according to their relationship with the commissural area. The anterolateral PM has a dual blood supply from branches of the circumflex and left anterior descending arteries. The posteromedial PM, in contrast, is supplied by a single artery arising from either the right coronary or from the terminal circumflex artery and is therefore more prone to infarction. Clinical studies have confirmed that CIMR is more likely to occur after posteroinferior than anterolateral $\mathrm{MI}^{51,52}$. However, the previously held notion that CIMR is due to isolated PM dysfunction is incorrect. Large animal studies have revealed that isolated PM infarction does not cause significant $\mathrm{MR}^{53}$, and may in fact paradoxically improve ischemic regurgitation ${ }^{54}$.

Left ventricular distortion and remodeling after MI displace PMs away from the mitral annulus ${ }^{24,51,55}$. The displacement puts excessive tension on the chordae, resulting in apical mitral leaflet tethering, restricting their coaptation during systole $23,24,31,52-56$. Leaflet tethering is compounded by LV contractile dysfunction, which decreases the closing force on the leaflets (Figure 1) ${ }^{23}$. Once IMR is initiated, end-diastolic LV volume and wall stress increase in tandem with preload ${ }^{10,32,52}$. Left ventricular mass also increases progressively without a concomitant increase in enddiastolic wall thickness ${ }^{57}$ resulting in generalized loss of myocardial contractile function $^{10,58}$. Increased wall stress causes more LV dysfunction ${ }^{59}$, which in turn results in further PM displacement and leaflet tenting. If LV dilation occurs, it leads to 
annular enlargement and dysfunction thereby increasing valvular incompetence ${ }^{60}$. Chronic IMR therefore begets MR in a self-perpetuating manner (Figure 2).

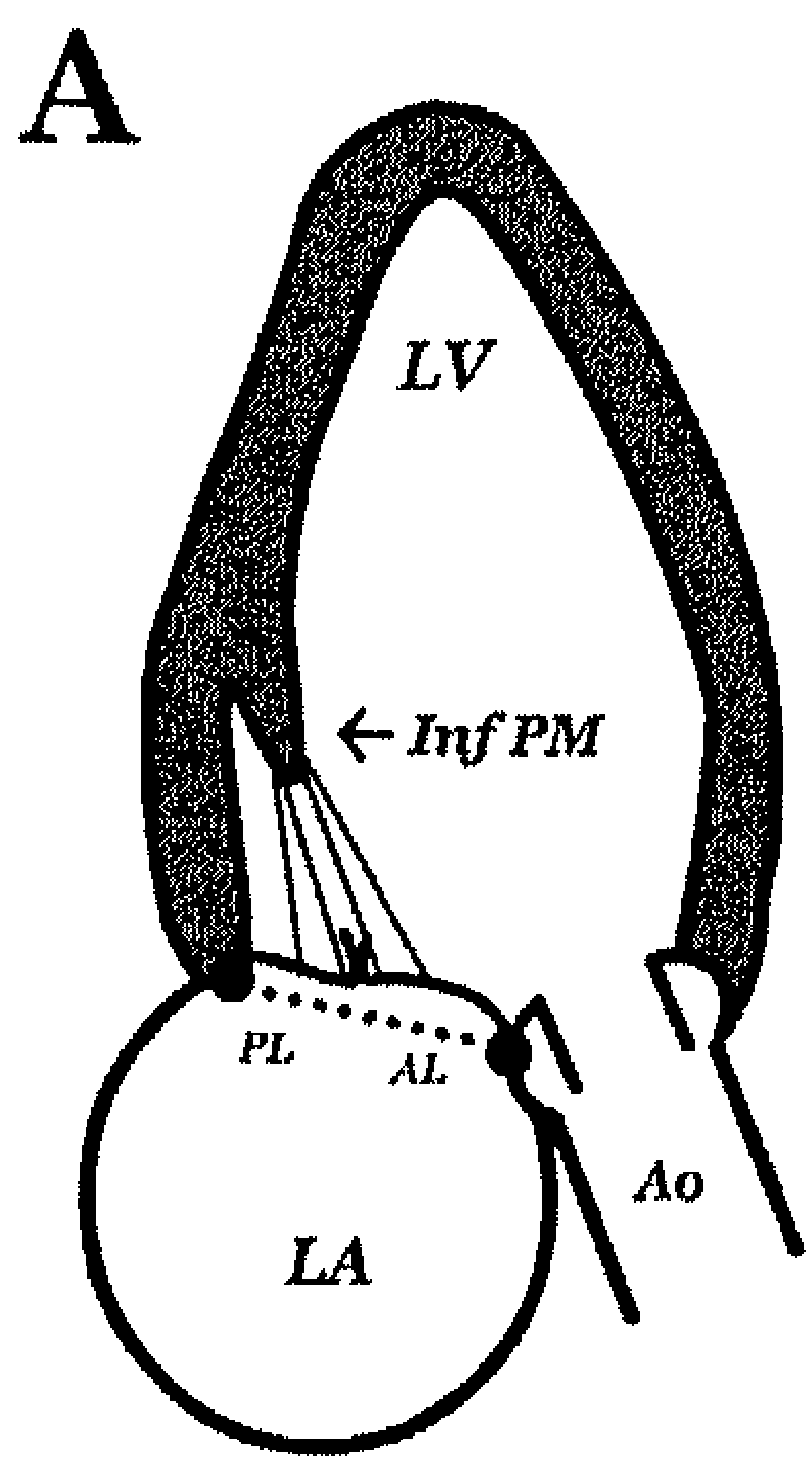

Normal

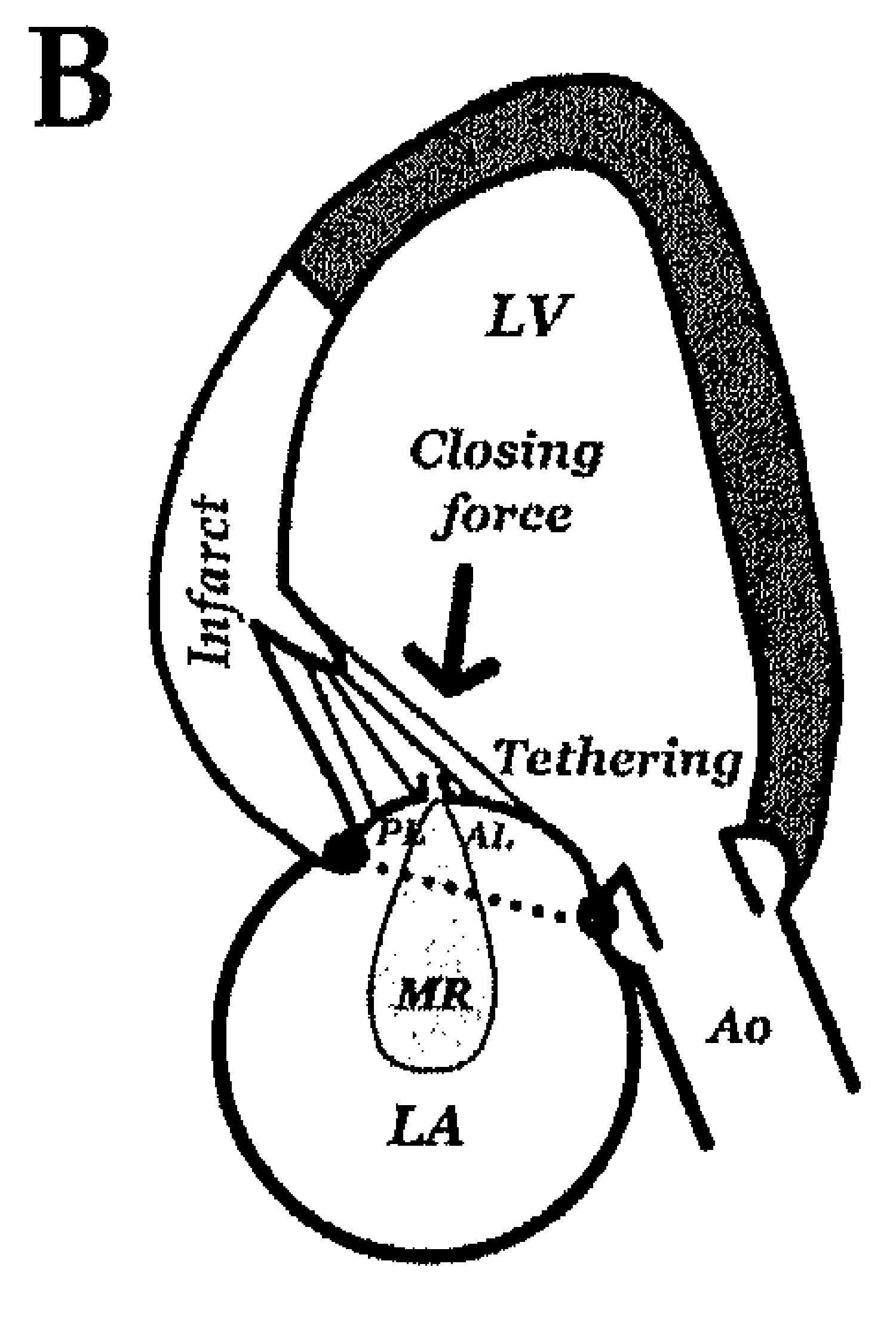

Infarct

Figure 1. In the Infarcted heart the displacement of papillary muscle results in tension on the chordae and tethering which lead to leaflet malcoaptation.

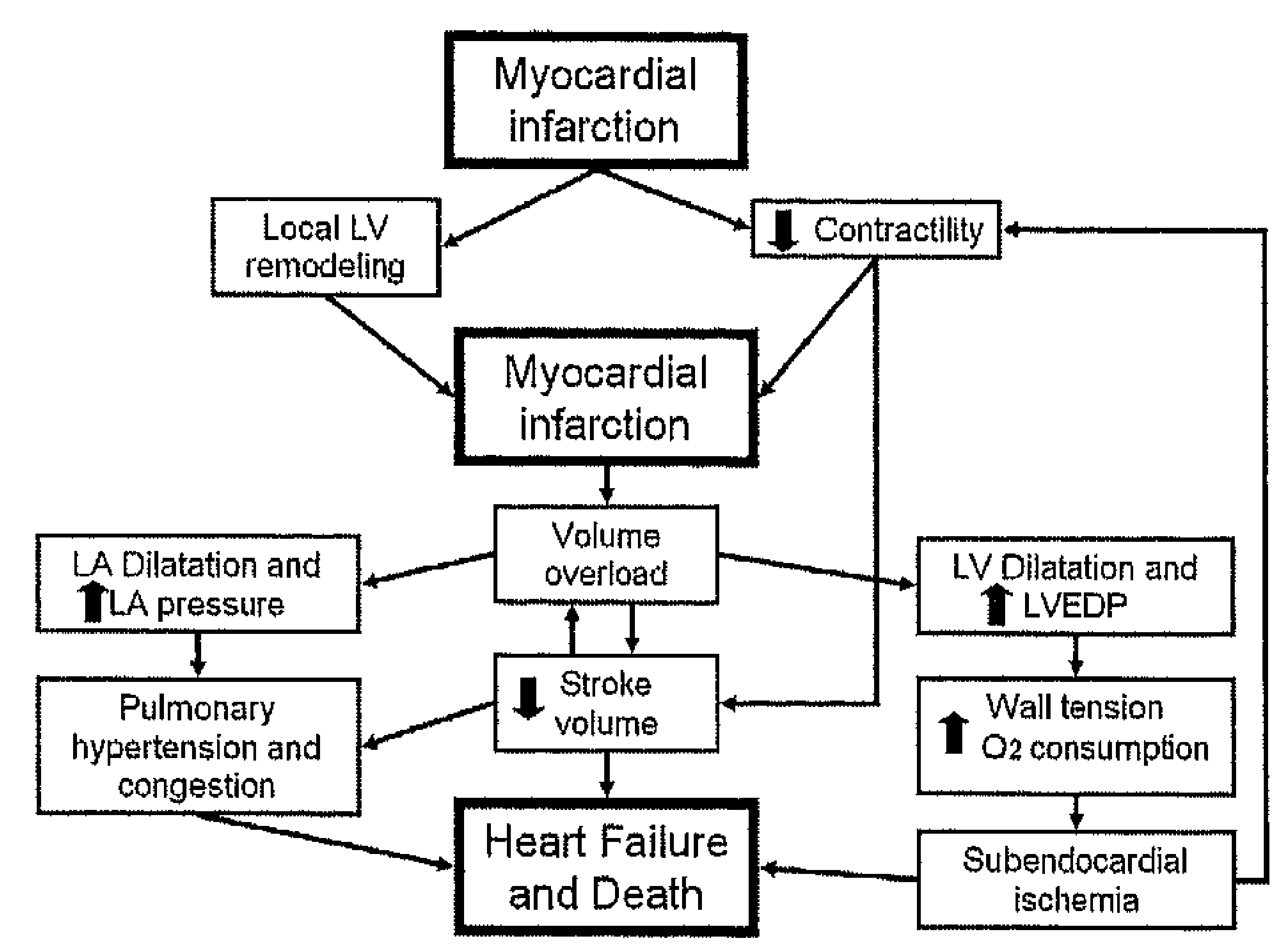

Figure 2. Self-maintaining mechanism leading to heart failure and death. 


\section{Clinical Examination}

The severity of symptoms (dyspnea, fatigue, pulmonary congestion) in patients with CIMR is mainly related to the underlying ventricular dysfunction. Therefore, ischemic MR is often unrecognized clinically. A murmur is heard only in a small percentage of patients, with estimates ranging from $4 \%$ to $50 \%{ }^{1,5,10,61-62}$. In contrast to structural mitral regurgitation, in ischemic mitral regurgitation the intensity of murmur is less and correlates poorly with the degree of regurgitation due to the decreased left ventricular systolic function and atrial compliance ${ }^{63}$. Indeed, in the non-compliant left atrium, the energy of the regurgitation created by a large effective regurgitant orifice cannot be transformed into kinetic energy which, in turn, produces a large regurgitant volume and a loud murmur. It is rather transformed into potential energy resulting in an increase of pressure as demonstrated by the high left atrial pressure and $\mathrm{V}$ wave ${ }^{7,64}$. Therefore, in ischemic mitral regurgitation, the regurgitant volume tends to be smaller and is associated with less intense murmur. However, this apparently "milder" regurgitation portends poor hemodynamics and worse outcomes, ${ }^{7,64}$. As a result, the clinician cannot rely on cardiac auscultation to diagnose it or assess its severity.

\section{Invasive investigations}

Left ventricular angiography is frequently associated with coronary angiography and some consider it as the reference method for the assessment of the volume of mitral regurgitation. This is mainly related to historical reasons, but grading using ventricular angiography is also subject to limitations, in particular due to the influence of loading conditions. Quantitative Doppler echocardiography provides more objective data regarding the prognostic role of the regurgitation than ventricular angiography.

\section{Echocardiography}

The limitations of the clinical exam in the detection of ischemic mitral regurgitation underscore the necessity of a test that is more sensitive than the mere bedside examination.

Historically, mitral regurgitation was assessed by left ventriculography ${ }^{1,10,65}$, relying on retrograde opacification of the left atrium but this technique does not provide adequate information on the anatomy of the valve and the etiology of the regurgitation. Echocardiography is the preferred method to assess valvular heart disease. The two-dimensional examination yields information on the geometry of the ventricles and valve anatomy, such as left ventricular dilatation, regional wall motion abnormalities, annulus dilatation, and especially valvular tenting. This provides information on the potential mechanisms of regurgitation which enables 
distinguishing ischemic mitral regurgitation from mitral regurgitation fortuitously associated with coronary artery disease. Standard color Doppler imaging is a highly sensitive method to detect even mild degrees of ischemic mitral regurgitation, and the measurement of the area of the regurgitant jet has been widely used to evaluate semi-quantitatively the amount of the regurgitation ${ }^{66}$. One unique advantage of echocardiography is that it accurately quantifies the severity of mitral regurgitation by measuring the effective regurgitant orifice area and the regurgitant volume using Doppler methodology (continuity equation or proximal isovelocity surface area [PISA], which is the preferred method). The effective regurgitant orifice area (EROA) is a measure of the severity of the lesion while the regurgitant volume (RV) is a measure of volume overload; therefore, the former is not dependent on hemodynamic conditions.

Current guidelines recommend thresholds to define severe mitral regurgitation as a regurgitant volume $\geq 60 \mathrm{ml}$ and an effective regurgitant orifice $\geq 40 \mathrm{~mm}^{67,68}$. For ischemic mitral regurgitation, however, adverse outcomes are associated with lower values for these parameters ${ }^{7}$ suggesting that $30 \mathrm{ml}$ for regurgitant volume and 20 $\mathrm{mm}^{2}$ for effective regurgitant orifice should be the preferred thresholds of severity.

The dynamic nature of ischemic mitral regurgitation was recently underscored. The exercise-induced increase in mitral regurgitation (effective regurgitant orifice change $>13 \mathrm{~mm}^{2}$ ) caused by changes in local remodeling was associated with increases in pulmonary pressure, pulmonary edema $a^{69}$, and cardiac death ${ }^{70}$. This may explain why small amounts of regurgitation at rest are associated with adverse outcomes. There are currently no data that suggest the optimal timing for imaging after myocardial infarction. This matter will require further studies.

\section{Prevalence of Chronic Ischemic Mitral Regurgitation}

The prevalence of CIMR has been difficult to calculate as a result of the heterogeneity of MR patients presented in previous studies ${ }^{49,51,71}$. Studies have reported a high incidence of IMR (11\% to 19\%) in patients undergoing cardiac catheterization for symptomatic $\mathrm{CAD}^{9,8,10}$. Hickey and colleagues ${ }^{10}$ projected the incidence of moderate-to-severe IMR to be $7 \%$ within this patient cohort. Approximately 14.6 million Americans have angina pectoris (chest pain) or a history of $\mathrm{MI}^{84}$. Combining the above statistics, it can be estimated that the prevalence of IMR in the United States is 1.6 million to 2.8 million patients ${ }^{9,10,72}$, a one-third increase from $1995^{49}$. These statistics show that CIMR continues to be a widespread problem and may increase as survival rates for acute $\mathrm{MI}$ improve. 
Patients with CIMR have a worse natural history than patients with CAD and no MR. A study of 11,748 cardiac catheterization patients revealed that severe ischemic MR was associated with a 1-year mortality of $40 \%$, moderate IMR $17 \%$, and mild IMR $10 \%{ }^{9}$. If no IMR was present at catheterization, the 1-year mortality rate was $6 \%$. Data from trials on thrombolysis for acute MI showed similarly poor prognosis for IMR'. Post-myocardial infarction patients have a 1-year mortality rate of $52 \%$ if they have severe IMR, $22 \%$ if they have mild-moderate IMR, and $11 \%$ if they have no ischemic MR.

The Survival and Ventricular Enlargement (SAVE) study' demonstrated that mild chronic IMR increases the risk of cardiovascular mortality, even in patients without congestive heart failure. Patients with CIMR had a higher incidence of cardiovascular mortality ( $29 \%$ versus $12 \%)$ and heart failure $(24 \%$ versus $16 \%)$ than patients without ischemic regurgitation at a mean of 3.5 years after MI. Adjustment for differences in baseline characteristics revealed that mild-to-moderate (1+ or 2+) CIMR strongly predicted mid-term mortality'.

\section{Medical Therapy and Cardiac Resynchronization}

The literature regarding medical therapy is comparatively sparse. Surprisingly, post-MI and heart failure patients commonly present with moderate MR despite the use of diuretics, $\mathrm{ACE}$ inhibitors, and $\beta$-adrenergic antagonists. Two questions arise: (1) Can medical therapy reduce regurgitation of a tethered valve? And (2) can it attenuate or reverse the underlying ventricular remodeling? First, recall the dynamic balance of tethering and closing forces that determines ischemic MR ${ }^{23,73,74}$. Transmitral pressure, which drives regurgitant flow, also promotes valve closure ${ }^{75}$. Therefore, it would appear that increased systemic blood pressure could reduce MR by increasing closing force. However, noninotropic vasopressors will increase afterload and LV volume, and therefore tethering and MR. Inotropic agents, in contrast, can raise LV pressure without increasing tethering, so MR will decrease, as with dobutamine infusion ${ }^{74,76}$; however, this is not a viable outpatient option. Transmitral pressure can also be increased by decreasing atrial pressure, so diuretics and nitrates can reduce regurgitant orifice area and flow ${ }^{77-80}$. Preload reduction and the initial decrease in MR can decrease ventricular size and further reduce tethering, creating a beneficial cycle. In severe heart failure, Stevenson and colleagues ${ }^{78}$ have shown that vasodilators and diuretics can partially reduce MR (from moderate to mild-to-moderate); Levine et a ${ }^{81}$ have uptitrated ACE inhibitors and nitrates to reduce MR partially. However, if afterload reduction is not accompanied by decreased PM tethering (for example, with fixed inferior wall scarring or a dilated, hypokinetic ventricle), regur- 
gitant orifice area may paradoxically increase as closing force decreases, as illustrated by Kizilbash, Grayburn, et al ${ }^{82}$ with nitroprusside.

Regarding the second question, acute LV decompression by vasodilators and diuretics is not the same as true reversal of the complex molecular, cellular, and interstitial remodeling process. Unfortunately, there are no strong data about ACE inhibitors and ischemic MR. In the SAVE (Survival And Ventricular Enlargement) study, Lamas et al ${ }^{1}$ found comparable cardiovascular mortality in patients with MR receiving captopril versus placebo; post-MI LV remodeling could only be partially attenuated by early ACE inhibition, but not reversed or completely prevented ${ }^{83-85}$. Ventricular dilatation and distortion may continue to progress beyond 1 year of therapy, and even those who do not initially dilate may "escape" later.

In SOLVD (Studies Of Left Ventricular Dysfunction), ACE inhibitors could arrest progressive LV dilatation ${ }^{86}$, and might even reverse $\mathrm{it}^{87}$, but there are no published data from large trials regarding whether attenuation of remodeling by ACE inhibitors or receptor blockers decreases the post-MI incidence of ischemic MR. MR appears to remain common despite use of these drugs and significantly increases morbidity and mortality.

Carabello and colleagues ${ }^{88,89}$ have firmly established that $\beta$-adrenergic receptor blockade can reverse the contractile and structural abnormalities caused by chronic nonischemic MR. Accordingly, in patients with chronic heart failure, $\beta$-blockade with carvedilol, when added to ACE inhibitors, can significantly decrease functional MR by reducing $L V$ dilatation ${ }^{89,91}$.

The CARMEN study (Carvedilol and ACE Inhibitor Remodeling Mild Heart Failure Evaluation Trial) $)^{92}$, with mainly ischemic cardiomyopathy, demonstrated synergistic reverse remodeling by carvedilol and enalapril in mild heart failure, and CAPRICORN (Carvedilol Post-Infarct Survival Controlled Evaluation) ${ }^{93}$ has recently shown incremental benefit of carvedilol over $\mathrm{ACE}$ inhibitors on ventricular remodeling after $\mathrm{MI}$ the effects on $\mathrm{MR}$, however, are unreported.

Tei et $\mathrm{a}^{94}$ have demonstrated a reduction in $\mathrm{LV}$ volume and MR in congestive heart failure with vasodilatation after warm bath and sauna, but chronic benefits require study. Limiting ventricular remodeling will ultimately be most effective early in its evolution. It remains to be seen whether vigorous combinations of vasodilator, $\beta$-blocking, and diuretic therapy, including spironolactone and other inhibitors of fibrosis and extracellular matrix degradation ${ }^{95}$, can limit the development of ischemic MR. Presently, such agents are not routinely administered to patients with inferior MI, whose localized remodeling may generate considerable MR. Practically, we must deal with the many patients with existing $M R$ and the incomplete effectiveness of current medical therapy.

A more favorable balance of forces on the valve can also be restored by electrical therapy in patients with heart failure. St. John Sutton, and colleagues ${ }^{96}$ have reported significantly decreased MR in the Multicenter InSync Randomized Clinical Evalu- 
ation. Biventricular pacing, with more effective LV contraction, can reduce endsystolic volume and therefore tethering. In heart failure, delayed lateral wall activation produces discoordinated PM contraction, malalignment of leaflet scallops, and MR; Kanzaki, Goresan, et a ${ }^{97}$ have shown that eliminating such delays by LV pacing reduces MR. Resynchronization also increases the leaflet closing force throughout systole, as shown by Breithardt, Sinha, Schwammenthal et $\mathrm{al}^{98}$ in patients with left bundle branch block; pacing can increase the rate of rise of LV pressure, thereby prolonging the time during systole when high LV and transmitral pressures oppose tethering to reduce regurgitant orifice area ${ }^{98}$, in direct correlation with $\mathrm{dP} / \mathrm{dt}$. Electrical therapy will therefore be most useful in those patients with cardiomyopathy whose $\mathrm{dP} / \mathrm{dt}$ rises and lateral wall conduction delay falls with pacing; its benefit extends to exercise, but with no evidence of benefit with preserved LV synchronicity or inferior MI.

\section{Surgical Treatment}

\section{Isolated $C A B G$ Versus $M V$ Surgery and $C A B G$}

The indications for surgery in chronic IMR are not well defined. It is generally agreed that patients who have indications for coronary artery bypass grafting (CABG) with moderate-to-severe CIMR (3+ or 4+) should also undergo concomitant MV surgery ${ }^{9 \%, 100}$. Rarely, patients present with episodic IMR that occurs only during episodes of acute ischemia. Such patients can be treated with revascularization alone with good results. It is controversial whether CABG patients with mild-to-moderate MR (1+ or 2+) should undergo concomitant MV surgery. Mallidi and coworker ${ }^{103}$ compared patients undergoing isolated CABG with mildto-moderate MR to patients without MR. They found a higher prevalence of heart failure symptoms and decreased cardiac event- free survival in MR patients during follow-up. In addition, $30 \%$ of patients progressed to $3+$ or $4+$ MR during a mean follow-up of 16 months.

Worsening of ischemic regurgitation after isolated CABG surgery has been demonstrated by other investigators and is associated with decreased long-term survival ${ }^{102,103}$. Aklog and coworkers ${ }^{102}$ found persistent moderate or severe MR in $77 \%$ of patients treated with revascularization alone and were unable to identify predictors of postoperative improvement. Wong and coworkers ${ }^{104}$ described more long-term MR, but no difference in survival, among patients with 3+ IMR who underwent isolated $C A B G$ versus patients who underwent $C A B G$ plus $M V$ repair. Such findings would suggest that MV surgery should be performed at the time of CABG in patients with mild-to-moderate IMR. However, the risk of long-term $M R$ and heart failure progression must be balanced against the increased perioperative risk of the additional MV procedure. Data from the STS database suggests that concomitant MV surgery increases the perioperative risk of CABG by roughly 
twofold ${ }^{101}$. We therefore recommend that patients with mild-to-moderate CIMR and multiple comorbidities, or a life expectancy of less than 5 years, should be treated conservatively and undergo CABG only. Low-risk patients with mild-to-moderate CIMR should undergo concomitant MV surgery provided the procedure can be performed with low mortality rates, namely, $5 \%$ or lower.

\section{Mitral Valve Replacement}

The preferred surgical approach to severe CIMR in early studies was MV replacement with a mechanical or tissue prosthesis. Mitral valve replacement involved complete excision of the subvalvular apparatus, but subsequent studies revealed a detrimental effect on LV function with this technique ${ }^{105}$. The negative effect on LV function may explain the high mortality rates in early CIMR surgical series. Preservation of the entire subvalvular apparatus can usually be performed in these patients because the MV leaflets and chordae are thin and pliable. Subvalvular preservation results in maintenance of annulo-ventricular continuity and improved preservation of LV function ${ }^{106}$. Mitral valve replacement is still a reasonable surgical option in many patients with CIMR, predominantly because of its reliability and reproducibility. Mitral valve replacement should be considered for patients with CIMR and multiple comorbidities, complex regurgitant jets (noncentral jet or more than one jet), or severe tethering of both MV leaflets ${ }^{71,107,108}$. Calafiore and coworkers ${ }^{107}$ recommend MV replacement when the distance between the coaptation point of the leaflets and the plane of the mitral annulus exceeds $10 \mathrm{~mm}$.

\section{Mitral Valve Repair}

The suboptimal early results for MV replacement led surgeons to start performing MV repair for CIMR. There have been no randomized trials to date comparing MV repair to replacement for chronic ischemic MR, but there have been a number of retrospective studies. The two largest and most methodologically sound studies are those performed by Gillinov and associates ${ }^{108}$ and Grossi and colleagues ${ }^{109}$. These investigators demonstrated that both MV repair and replacement are effective at eliminating MR immediately postoperatively, but MV repair was associated with lower perioperative mortality. However, patients at the highest risk with the most severe MR did just as well, and possibly better, with MV replacement ${ }^{71,108}$. Regardless of which surgical technique was employed, 5-year survival rates were uniformly disappointing at approximately $50 \%{ }^{108,109}$.

Currently, there is general consensus in the cardiac surgery community that MV repair results in lower perioperative mortality than MV replacement and should therefore be performed whenever possible ${ }^{107}$. A recent study by Al-Radi and coworkers ${ }^{10}$ confirmed a lower perioperative mortality from MV repair in patients with CIMR. The survival advantage decreased over time, however, and was no longer apparent by 5 years after surgery. 
The most common surgical procedure currently performed for CIMR is undersized mitral ring annuloplasty (UMRA). Bolling and colleagues" ${ }^{11}$ popularized this approach, using very small (size 24 to 26 ) mitral annuloplasty rings. The rationale is that undersizing the mitral annulus will result in increased leaflet coaptation and decreased regurgitation. Although this procedure does not fully address the ventricular causes of CIMR, it is simple to perform and reproducible, and therefore has been widely adopted by the cardiac surgery community. The type of ring that should be used for UMRA is unclear, with several authors arguing the merits of rigid versus flexible and complete versus incomplete rings ${ }^{12}$. A close examination of the literature does not reveal any definite advantages for any specific type of annuloplasty ring, however, with the exception of poor long-term results for pericardial bands ${ }^{113}$. The initial results for undersized mitral annuloplasty seemed encouraging, with low perioperative mortality rates ${ }^{114}$. However, subsequent studies at various centers revealed a significant proportion of patients develop recurrent $M R$ during follow-up. A recent article from the Cleveland Clinic examined 585 patients undergoing undersized annuloplasty surgery over a 17 -year period ${ }^{115}$. In $28 \%$ of patients, moderate or more MR developed 6 months postoperatively, an incidence that is similar to that described by other investigators ${ }^{116,117}$. Mitral regurgitation recurrence tends to occur early postoperatively with relatively low recurrence rates thereafter ${ }^{113}$. The relatively high MR recurrence rate associated with UMRA has led a number of investigators to examine alternative surgical therapies.

Recently, the issue of annulus re-shaping during annuloplasty has been addressed by some authors ${ }^{118}$. In contrast with rigid/semi-flexible plane annuloplasty rings which may flatten the natural saddle shape causing greater tension on chordae and leaflets, innovative annular rings mimic the shape of healthy mitral annulus ${ }^{119,120}$.

\section{Alternative Surgical Procedures}

Messas and coworkers ${ }^{3}$ proposed to reduce leaflet tethering by cutting a limited number of critically positioned second-order chordae tendineae. Secondary chords are the most responsible chordae for leaflet restriction in CIMR, but are not required to prevent leaflet prolapse (Figure 3). Dividing secondary chordae in a sheep model of CIMR resulted in improved leaflet coaptation and reduced MR, without leaflet prolapse or decline in LV ejection fraction ${ }^{3}$. Other investigators have demonstrated, however, that second-order chordae cutting may adversely affect LV function ${ }^{121}$. A recent case report described no MR and improved LV function in a patient undergoing chordal cutting surgery ${ }^{122}$. The Group of Tirone David ${ }^{123}$ has recently adopted the technique of chordal cutting for patients with CIMR. They divide all secondorder chords arising from the affected PM (usually the posteromedial muscle) at their insertion on the anterior and posterior leaflets, and insert a slightly undersized (size 28 to 30) flexible posterior annuloplasty ring. They have performed this operation in more than 30 patients, with a perioperative mortality rate of $6 \%$. Follow- up 


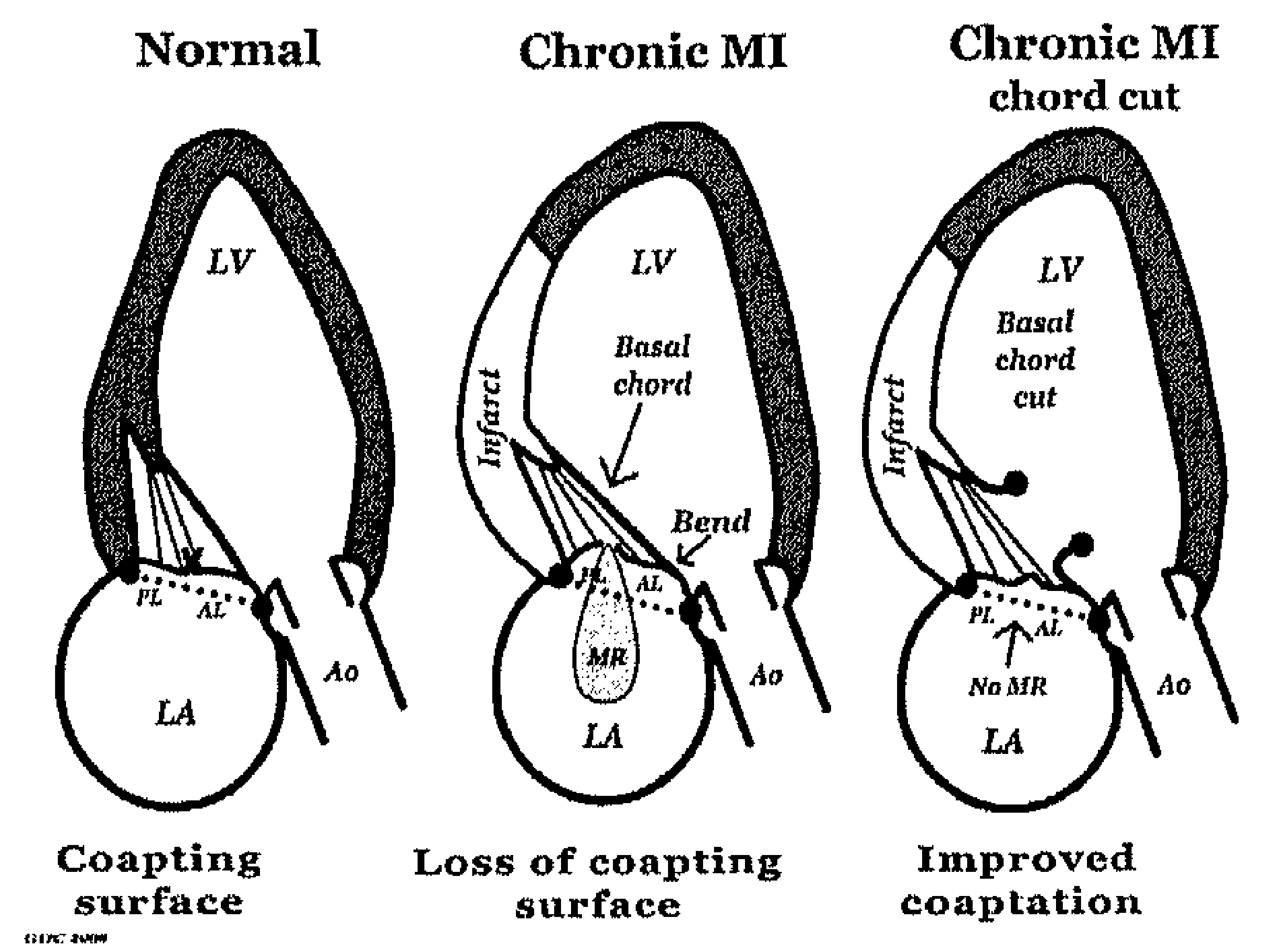

Figure 3. Secondary chordae cut may improve leaflet coaptation.

A

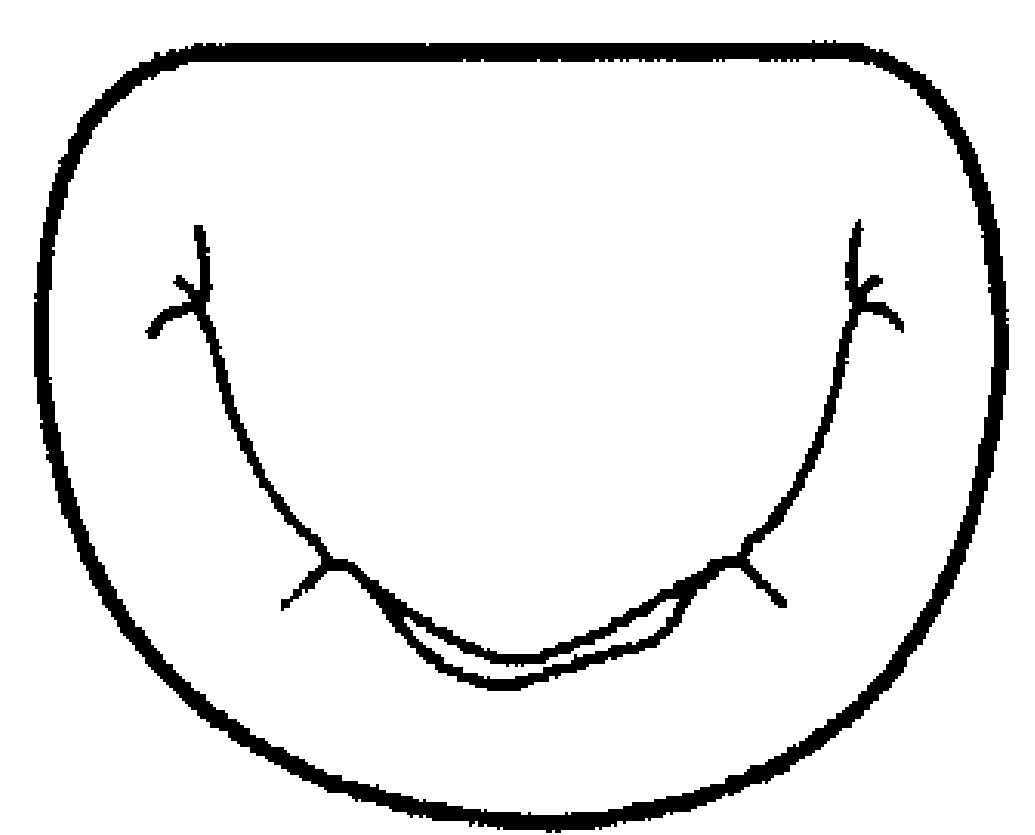

Figure 4. Alfieri's edge-to edge correction: creation of a double orifice.

echocardiography revealed trivial or mild MR in $97 \%$ of patients for as long as 24 months postoperatively. In addition, there has been no decrease in LV function in the early postoperative period. Although we are enthusiastic about this procedure at this time, the long-term results and durability of the repair remain to be seen.

Alfieri and colleagues ${ }^{124,125}$ have described an edge-to-edge MV leaflet repair technique for many different causes of MR (Figure 4). The technique is quick and relatively simple to perform. A suture is used to join the center of the anterior and posterior leaflets, creating a double orifice MV. A mitral annuloplasty ring is inserted because long-term results without an annuloplasty ring are suboptimal ${ }^{125}$. Although many studies have indicated good results for the Alfieri repair technique, most had small numbers of patients with CIMR. An exception is the recent study by Bhudia and colleagues ${ }^{126}$ that included 143 patients with ischemic cardiomyopathy. These investigators found a progressive increase in MR recurrence in CIMR patients, with more than $30 \%$ of patients having moderate-to-severe MR 1 year postoperatively. 
They concluded that other MV repair techniques are required for this patient population.

Liel-Cohen and associates ${ }^{30}$ devised an infarct placation procedure to reverse $\mathrm{LV}$ remodeling in sheep. The infarcted region of the $\mathrm{LV}$ is plicated with mattressed sutures to reduce myocardial bulging and to bring the displaced PM tips back toward the anterior mitral annulus (Figure 5).

The plication process also reduces the proportion of LV circumference occupied by infracted myocardium. Infarct plication for CIMR was recently described in humans. Ramadan and colleagues ${ }^{127}$ performed plication of posterolateral myocardial scar without mitral annuloplasty in 3 patients. Two patients had no MR and 1 had trivial MR 7 months postoperatively. Although this technique appears promising, more data are required before it can be recommended for CIMR patients.

Menicanti and associates ${ }^{128}$ devised a LV restoration procedure for the treatment of CIMR and dilated ischemic cardiomyopathy. The procedure consists of coronary revascularization, LV restoration with a Dor procedure, and imbrication of the PMs without a prosthetic ring (Figure $6 \mathrm{~A}, \mathrm{~B}$ ). The Dor procedure may reduce $\mathrm{MR}$ in dilated ischemic cardiomyopathy by reducing LV size and improving PM orientation ${ }^{129}$. These Authors have used this approach in 46 patients with previous anterior transmural MI and MR. Postoperative MR was mild or less in $84 \%$ of the patients.

Another complex surgical approach to CIMR, the papillary muscle sling, has been recently described by Hvass and colleagues ${ }^{130}$. This technique consists of correcting abnormal PM displacement with an intraventricular Gore-Tex sling. The device is implanted through the left atrium and across the mitral orifice, encircling the trabecular base of both PMs (Figure 7). Tightening the sling with sutures decreases the distance between the PMs, and a moderately undersized mitral annuloplasty ring is inserted. A double ring is thereby produced: one inside the ventricle and one on the mitral annulus. These Authors have applied this technique in 10 patients, with reduced mitral tenting and decreased $M R$ in all patients immediately postoperatively. However, the long-term effects of this technique are unknown.

Kron and associate ${ }^{60}$ have described another technique, the surgical re-location of the posterior papillary muscle, for the treatment of CIMR, particularly in patients with severe restriction of the $\mathrm{P} 3$ segment of the mitral valve. A suture is used to connect the posterior PM to the mitral annulus, adjacent to the right fibrous trigone, and a mitral annuloplasty ring is inserted (Figure 8). The suture between the PM and the mitral annulus is shortened to alleviate tethering of the P3 segment and to increase leaflet coaptation. These authors have performed this procedure in 18 patients, with all patients having no or trace MR eight weeks postoperatively.

Finally, Fundaro and associates ${ }^{131}$ devised a relatively simple technique to ameliorate posterior leaflet tethering and restore normal distance between the annulus and PM (posterior mitral valve restoration). An incision is made at the base of the posterior leaflet and the basal chordae are transected to increase posterior leaflet 


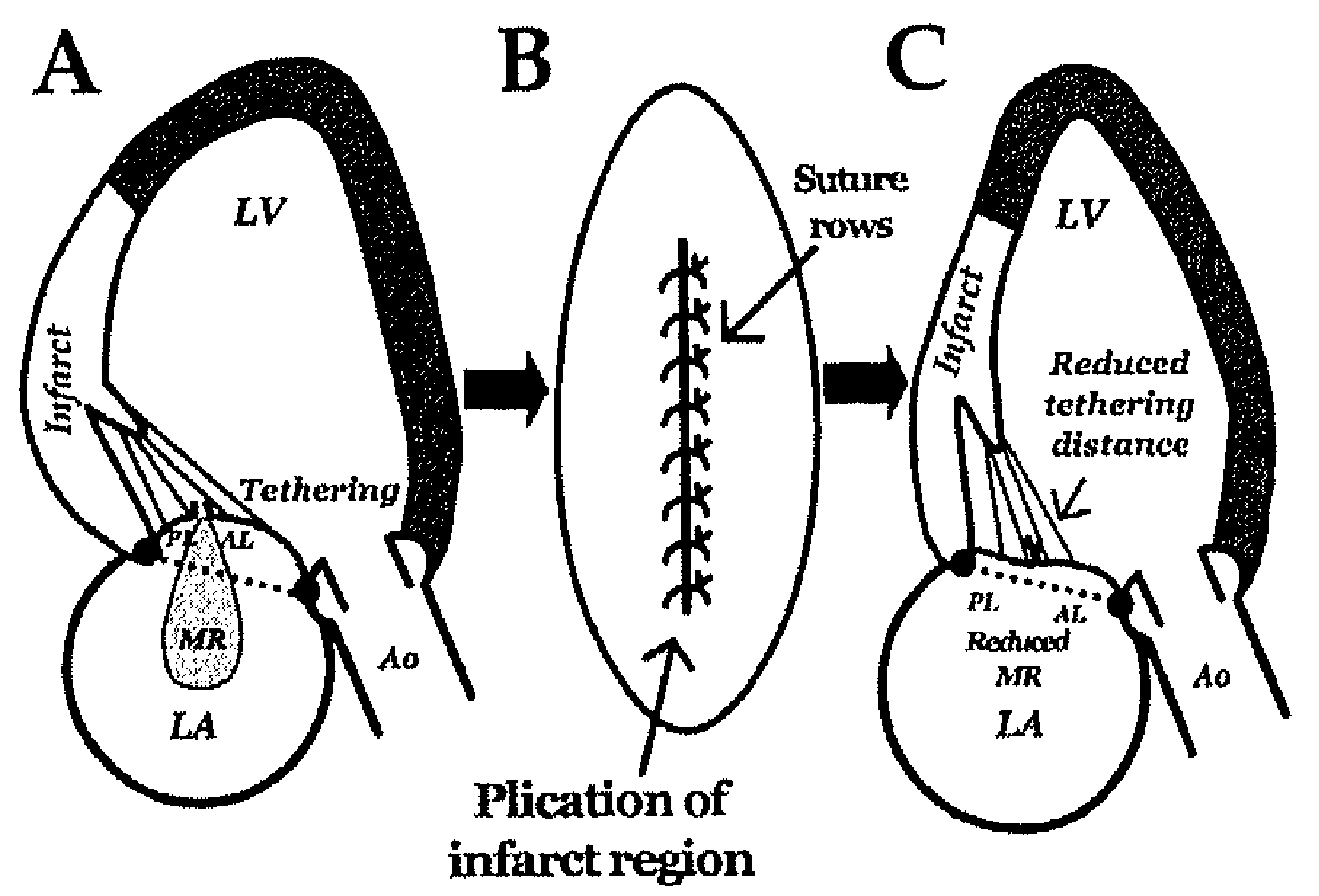

Figure 5. Left Ventricular plication reduces left ventricular bulging and papillary muscle displacement.

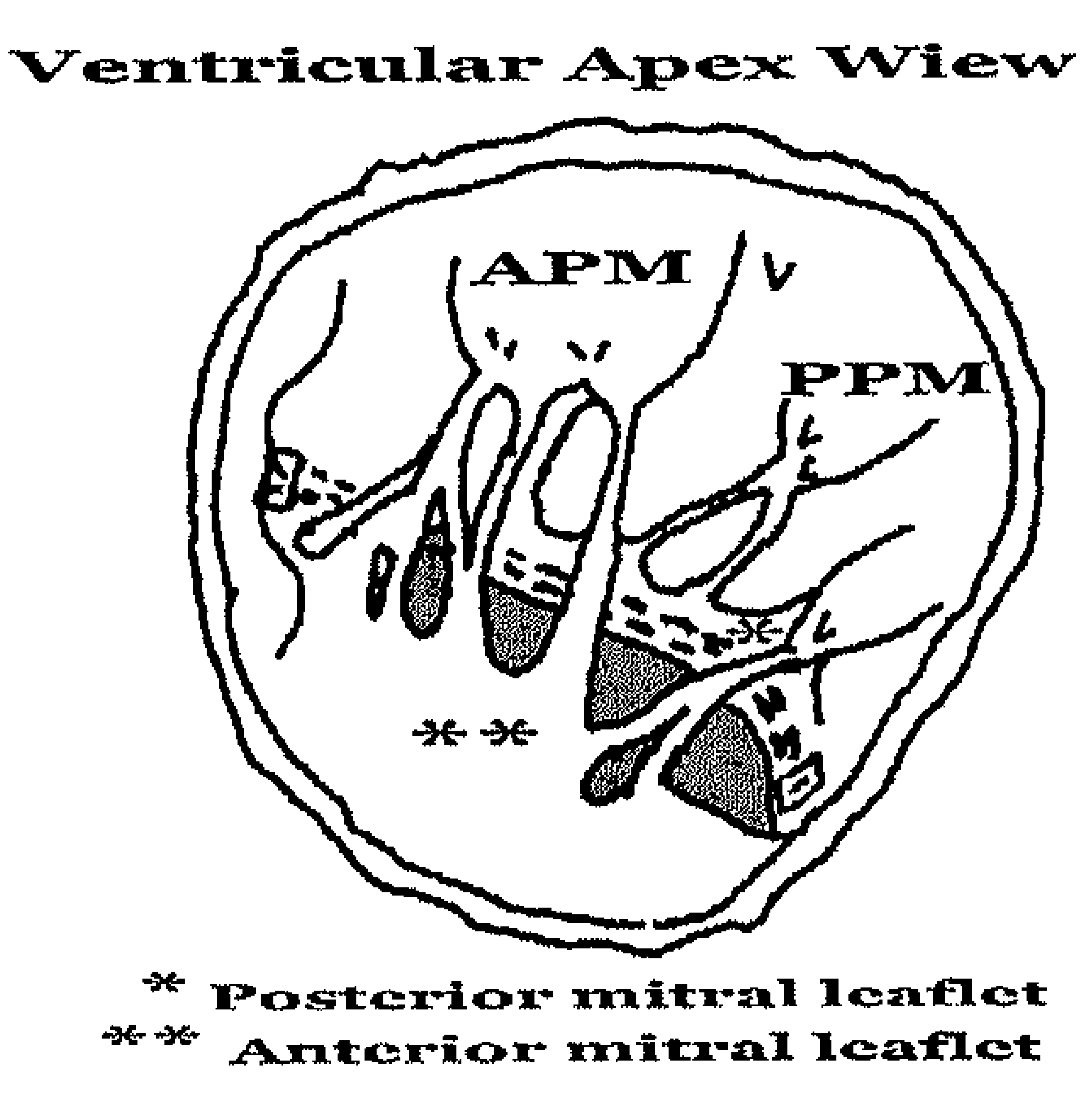

Figure 6. Papillary muscle imbrication: a $2-0$ polyester double-armed suture with a pledged is applied from the ventricular side to atrial side. The two arms of this stitch with a running suture were conducted toward the left trigone. The stitch runs a few millimeters from the mitral annulus biting atrial and ventricular muscle. The posterior annulus is completely bounded by this suture. The left trigone is reached, and the two arms of the suture are conducted from the atrial side to the ventricular side through the trigone just below the aortic valve. A $23-\mathrm{mm}$ sizer is introduced into the mitral valve to create an undersized orifice but leave a surface area bigger than $3 \mathrm{~cm}^{2}$, and the suture is tied over a second pledged. Then the ventricular restoration is performed.

mobility. The detached portion of the mitral annulus is then plicated and the resulting defect in the posterior leaflet is closed with a running suture. The plicated annulus is reinforced with a short Gore-Tex strip or posterior annuloplasty band (Figure 9). The data on this procedure are poor.

\section{Experimental Procedures}

The Alfieri MV repair technique has recently been applied percutaneously ${ }^{132}$. A double-armed clip device, deployed through the femoral vessels, was developed in a large animal model and is now undergoing trials in patients. The results for 


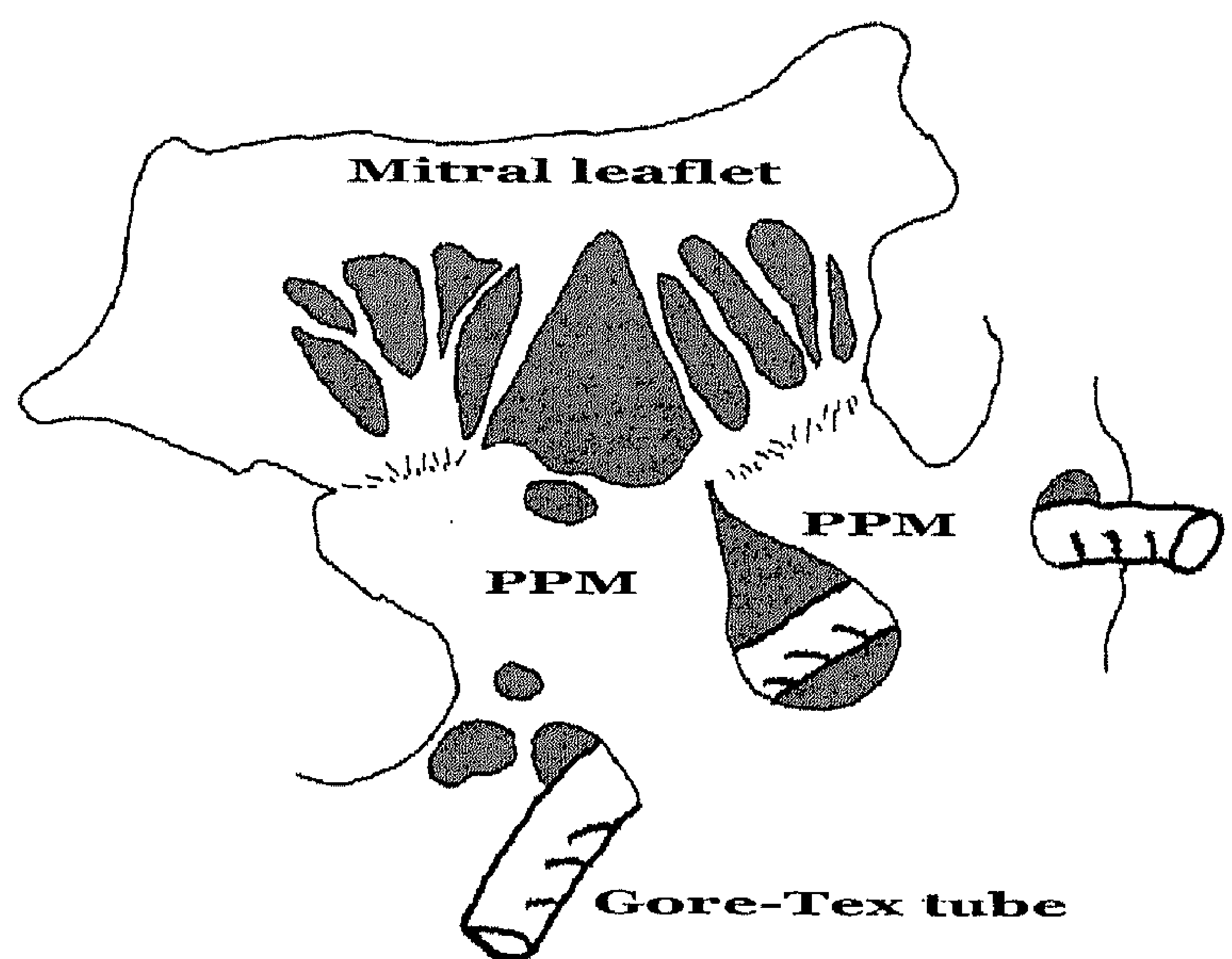

Figure 7. Papillary muscle sling: a Gore-Tex sling is placed around the trabecular base of both papillary muscles.

A

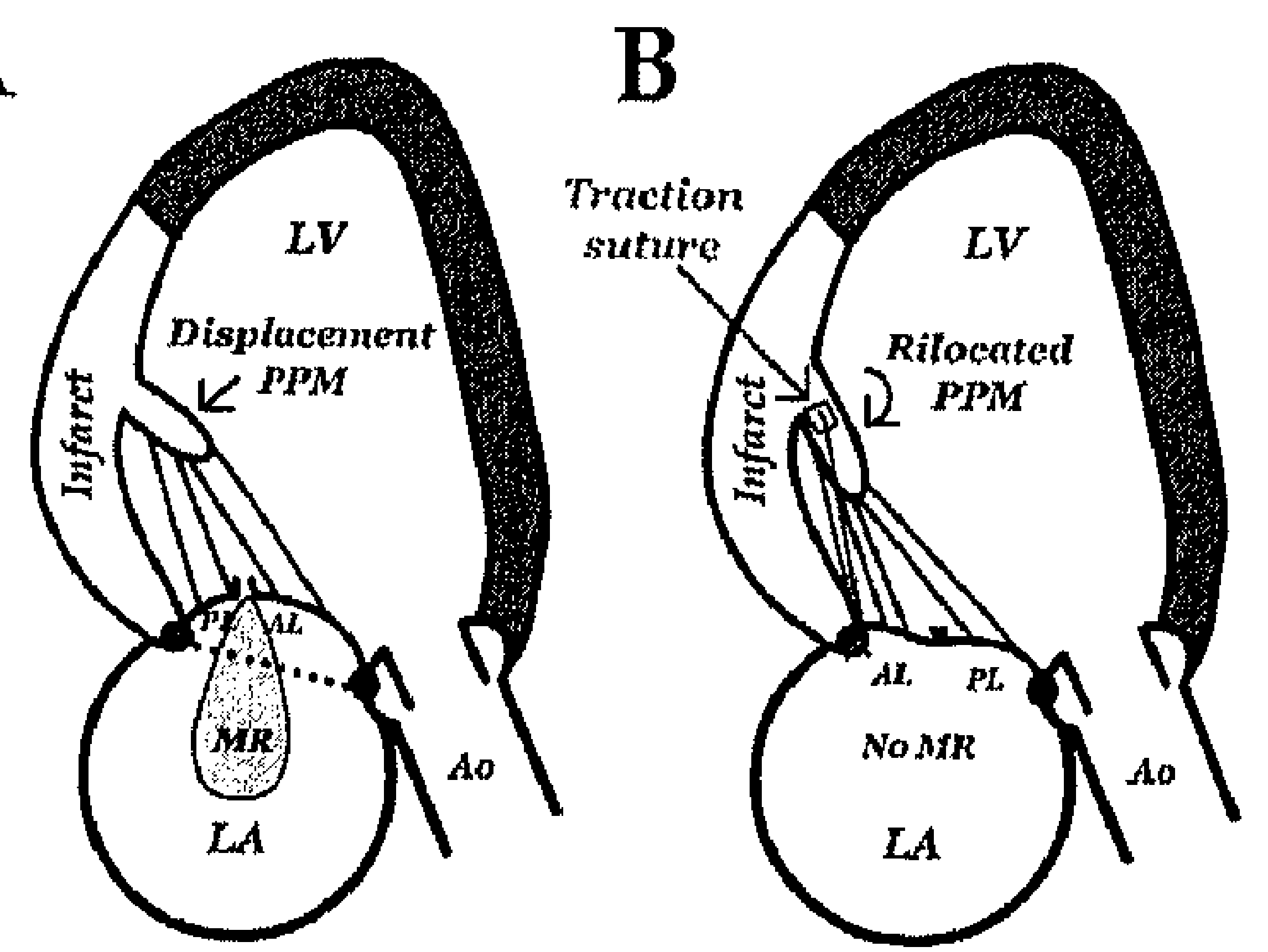

Figure 8. Surgical Relocation of the posterior papillary muscle: the posterior papillary muscle is connected by a pledged suture to the mitral annulus.

this device are pending, but MR recurrence rates may be higher compared with the conventional Alfieri operation because a mitral annuloplasty ring is not inserted ${ }^{124}$.

Percutaneous mitral annuloplasty has been recently described in large animal models of CIMR by two separate groups. Devices were inserted percutaneously into the coronary sinus in sheep $\mathrm{p}^{133}$ and $\operatorname{dog}^{134}$. Both studies revealed acute reductions in MR, but long-term animal studies are pending. Percutaneous annuloplasty offers the advantage of avoiding an operation, but has the potential disadvantage of coro- 


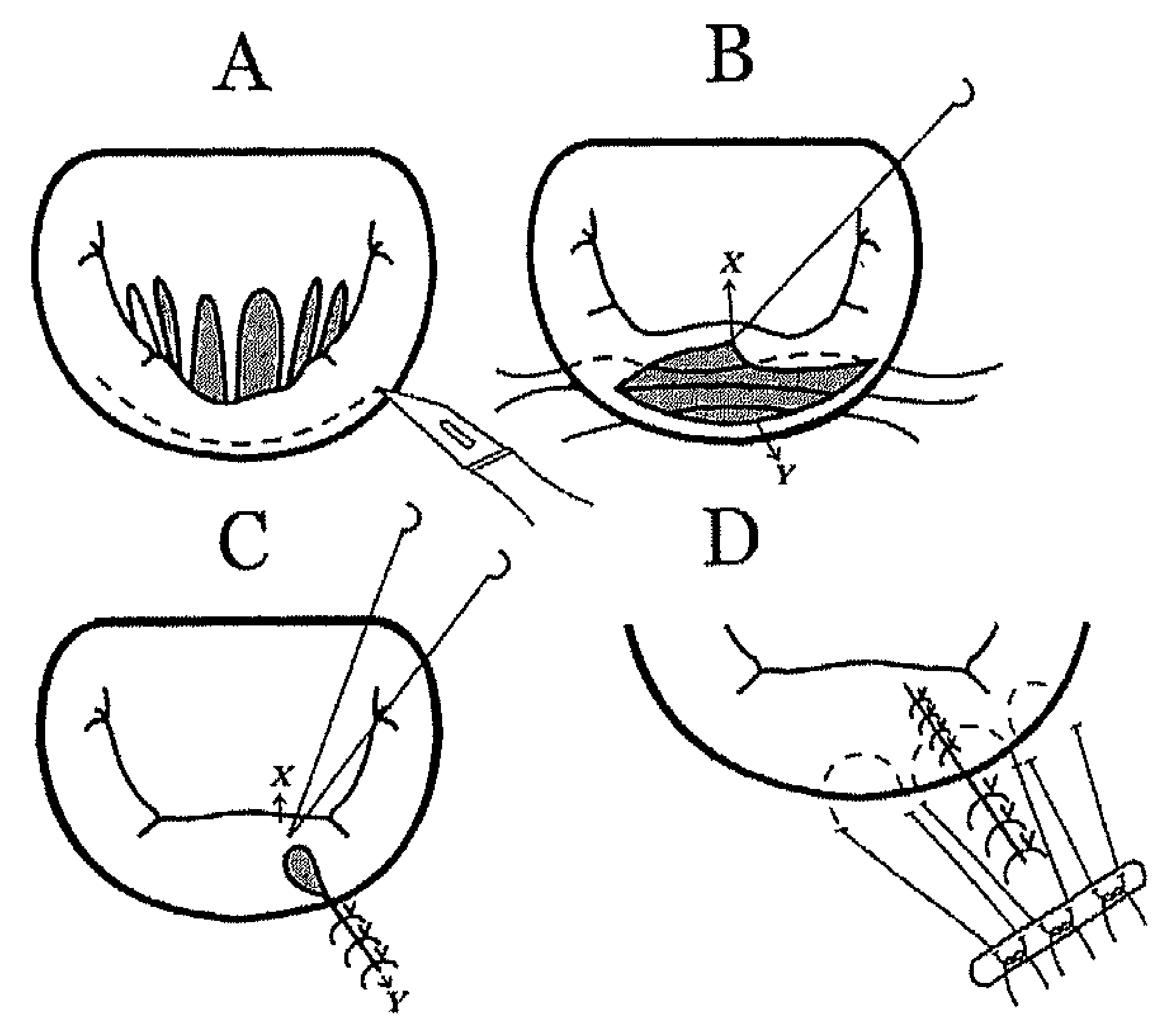

Figure 9. Transection of basal chordae through an incision of the posterior mitral leaflet (A-B). This is followed by a plication of the annulus $(\mathrm{C})$ and posterior annuloplasty with a Gore-Tex strip (D).

nary sinus perforation or thrombosis, or injury to the adjacent circumflex artery ${ }^{135}$. In addition, the durability of this approach may be compromised by the coronary sinus having no fibrous connections to the mitral annulus.

Septolateral annular cinching was recently used by Tibayan and colleagues ${ }^{136}$ in an ovine model of CIMR. Septolateral annular cinching is performed by anchoring a single suture to the midseptal annulus, then exteriorizing the suture through the posterior lateral annulus. The suture is tightened under echocardiographic guidance to reduce lateral displacement of the posterior PM. As opposed to undersized ring annuloplasty, septolateral annular cinching is not associated with impaired mobility of either the anterior or posterior leaflets ${ }^{137}$. Septolateral annular cinching decreased ischemic MR and reduced the septolateral diameter in sheep ${ }^{136}$, but clinical studies are pending.

The Coapsys device (Myocor, Maple Grove, Minnesota) is designed to restore septolateral annular geometry in patients with IMR ${ }^{138}$. It is inserted through a sternotomy but without cardiopulmonary bypass. The device consists of two epicardial pads and an expanded polytetrafluoroethylene- coated subvalvular chord. The posterior pad has two heads that change the shape of the LV at the mitral annulus and PM level. The anterior pad is adjustable and is fixed after echocardiographically confirmed positioning of the posterior pad. The Coapsys device has been successfully used in a dog model ${ }^{140}$, and is currently undergoing clinical assessment. 
The CorCap Cardiac Support Device (CSD) (Acorn Cardiovascular, Inc., St. Paul, Minnesota) ${ }^{139,140}$ is a mesh-like device that is surgically implanted around the heart. The device is designed to provide circumferential myocardial wall support, and reduce wall stress and myocyte stretch.

In a recent study $y^{140}$ it has been implanted in 24 patients with dilated cardiomyopathy, severe LV dysfunction, and advanced heart failure (NYHA class II-IV) at four centers in Italy and Sweden.

Investigators demonstrated improvements in cardiac structure and function as well as in patient functional status after CorCap CSD implantation. Nonetheless its efficacy in CIMR is still uncertain. Finally, Hung and associates ${ }^{2}$ devised a Dacron patch inflatable balloon to reverse LV remodeling and reduce IMR. The balloon device is secured externally onto the infarcted myocardium and is inflated under echocardiographic guidance. The infarcted myocardium and PM is displaced inward toward the anterior mitral annulus, thereby decreasing leaflet tethering. The inflatable balloon has the benefit of not requiring cardiopulmonary bypass, but is yet to be tested in humans. 
1. Lamas GA, Mitchell GF, Flaker GC, et al. for the Survival and Ventricular Enlargement Investigators. Clinical significance of mitral regurgitation after acute myocardial infarction. Circulation 1997;96 (3):827-33.

2. Hung J, Guerrero JL, Handschumacher BS, Supple G, Sullivan S, Levine RA. Reverse ventricular remodeling reduces ischemic mitral regurgitation: echo-guided device application in the beating heart. Circulation 2002;106:2594-600.

3. Messas E, Pouzet B, Touchot B, et al. Efficacy of chordal cutting to relieve chronic persistent ischemic mitral regurgitation. Circulation 2003;108(Suppl 2):111-5.

4. Barzilai B, Gessler C, Perez JE, et al. Significance of Doppler detecred mitral regurgitation in acute myocardial infarcrion. Am J Cardiol 1988;61:220 -3.

5. Lehmann KG, Francis CK, Dodge HT, and the TIMI Study Group. Mitral regurgitation in early myocardial infarction: incidence, clinical detection, and prognostic implications. Ann Intern Med 1992;117:10-7.

6. Grigioni F, Enriquez-Sarano M, Zehr KJ, Bailey KR, Tajik AJ. Ischemic mitral regurgitation: long-term outcome and prognostic implications with quantitative Doppler assessment.Circulation 2001;103 (13):1759-64.

7. Trichon $\mathrm{BH}$, Felker GM, Shaw LK, Cabell CH, O'Connor CM. Relation of frequency and severity of mitral regurgitation to survival among patients with left ventricular systolic dysfunction and heart failure. Am J Cardiol 2003;91:538-43.

8. Hickey MS, Smith LR, Muhlbaier LH, et al. Current prognosis of ischemic mitral regurgitation: implications for future management. Circulation 1988;78(Suppl 1):I-51-9.

9. Tcheng JE, Jackman JD Jr, Nelson CL, et al. Outcome of patients sustaining acute ischemic mitral regurgitation during myocardial infarction. Ann Intern Med 1992;117:18 -24.

10. Frantz E, Weininger $F$, Oswald $H$, Fleck E. Predictors for mitral regurgitation in coronary artery disease. In: Vetter HO, Hetzer R, Schmutzler H, eds. Ischemic mitral incompetence. New York: Springer-Verlag, 1991:57.

11. Kishon Y, Oh JK, Schaff HV, Mullany CJ, Tajik AJ, Gersh BJ. Mitral valve operation in postinfarction rupture of a papillary muscle: immediate results and long-term follow-up of 22 patients. Mayo Clinic Proceedings 1992; 67(11): 1023-30.

12. Birnbaum Y, Chamoun AJ, Conti VR, Uretsky BF. Mitral regurgitation following acute myocardial infarction. Coron Artery Dis 2002;13(6): 337-44.

13. Burch GE, DePasquale NP, Phillips JH. Clinical manifestations of papillary muscle dysfunction. Arch Intern Med. 1963;112:112-117.

14. Burch GE, DePasquale NP, Phillips JH. The syndrome of papillary muscle dysfunction. Am Heart J. 1968;75:399-415.

15. Mittal AK, Langston M Jr, Cohn KE, Selzer A, Kerth WJ. Combined papillary muscle and left ventricular wall dysfunction as a cause of mitral regurgitation: an experimental study. Circulation. 1971;44:174-180. 
16. Silverman ME, Hurst JW. The mitral complex: interaction of the anatomy, physiology, and parhology of the mitral annulus, mitral valve leaflets, chordae tendineae, and papillary muscles. Am Heart J. 1968;76:399-418.

17. Perloff JK, Roberts WC. The mitral apparatus: functional anatomy of mitral regurgitation. Circulation. 1972;46:227-239.

18. Ogawa S, Hubbard FE, Mardelli TJ, Dreifus LS. Cross-sectional echocardiographic spectrum of papillary muscle dysfunction. Am Heart J.1979;97:312-321.

19. Godley RW, Wann LS, Rogers EW, Feigenbaum H, Weyman AE.Incomplete mitral leaflet closure in patients with papillary muscle dysfunction. Circulation. 1981;63:565-571.

20. Kaul S, Spotnitz WD, Glasheen WP, Touchstone DA. Mechanism of ischemic mitral regurgitation: an experimental evaluation. Circulation.1991;84:2167-2180.

21. Dent JM, Spotnitz WD, Nolan SP, Jayaweera AR, Glasheen WP, KaulS. Mechanism of mitral leaflet excursion. Am J Physiol. 1995;269:H2100-H2108.

22. He S, Fontaine AA, Schwammenthal E, Yoganathan AP, Levine RA. Integrated mechanism for functional mitral regurgitation: leaflet restriction versus coapting force: in vitro studies. Circulation. 1997;96:1826-1834.

23. Otsuji Y, Handschumacher MD, Schwammenthal E, Jiang L, Song JK, Guerrero JL, Vlahakes GJ, Levine RA. Insights from three-dimensional echocardiography into the mechanism of functional mitral regurgitation: direct in vivo demonstration of altered leaflet tethering geometry. Circulation. 1997;96:1999 -2008.

24. Kono T, Sabbah HN, Rosman H, Alam M, Jafri S, Goldstein S. Left ventricular shape is the primary determinant of functional mitral regurgitation in heart failure. J Am Coll Cardiol. $1992 ; 20: 1594-1598$.

25. Sabbah HN, Rosman H, Kono T, Alam M, Khaja F, Goldstein S. On the mechanism of functional mitral regurgitation. Am J Cardiol. 1993;72:1074-1076.

26. Sabbah HN, Kono T, Stein PD, Mancini GB, Goldstein S. Left ventricular shape changes during the course of evolving heart failure.Am J Physiol. 1992;263:H266-H270.

27. Otsuji Y, Handschumacher MD, Liel-Cohen N, Tanabe H, Jiang L, Schwammenthal E, Guerrero JL, Nicholls LA, Vlahakes GJ, Levine RA. Mechanism of ischemic mitral regurgitation with segmental left ventricular dysfunction: three-dimensional echocardiographic studies in models of acute and chronic progressive regurgitation. J Am Coll Cardiol. 2001;37:641- 648.

28. Llaneras MR, Nance ML, Streicher JT, Lima JA, Savino JS, Bogen DK, Deac RF, Ratcliffe MB, Edmunds LH Jr. Large animal model of ischemic mitral regurgitation. Ann Thorac Surg. 1994;57:432- 439 .

29. Gorman RC, McCaughan JS, Ratcliffe MB, Gupta KB, Streicher JT, Ferrari VA, St John-Sutton $M G$, Bogen DK, Edmunds LH Jr. Pathogenesis of acute ischemic mitral regurgitation in three dimensions. J Thorac Cardiovasc Surg. 1995;109:684-693.

30. Liel-Cohen N, Guerrero JL, Otsuji Y, Handschumacher MD, Rudski LG, Hunziker PR, Tanabe H, Scherrer-Crosbie M, Sullivan S, Levine RA. Design of a new surgical approach for ventricular remodeling to relieve ischemic mitral regurgitation: insights from 3-dimensional echocardiography.Circulation. 2000;101:2756 -2763. 
31. Yiu SF, Enriquez-Sarano M, Tribouilloy C, Seward JB, Tajik AJ. Determinants of the degree of functional mitral regurgitation in patients with systolic left ventricular dysfunction: a quantitative clinical study. Circulation. 2000;102:1400-1406.

32. Kwan J, Shiota T, Agler DA, Popovic ZB, Qin JX, Gillinov MA, Stewart WJ, Cosgrove DM, McCarthy PM, Thomas JD. Geometric differences of the mitral apparatus between ischemic and dilated cardiomyopathy with significant mitral regurgitation: real-time three-dimensional echocardiography study. Circulation. 2003;107:1135-1140.

33. Lai DT, Tibayan FA, Myrmel T, Timek TA, Dagum P, Daughters GT, Liang D, Ingels NB Jr, Miller DC. Mechanistic insights into posterior mitral leaflet inter-scallop malcoaptation during acute ischemic mitral regurgitation. Circulation. 2002;106(suppl I):I40 -145.

34. Boltwood CM, Tei C, Wong M, Shah PM. Quantitative echocardiography of the mitral complex in dilated cardiomyopathy: the mechanism of functional mitral regurgitation. Circulation. 1983;68:498 -508.

35. Kisanuki A, Minagoe S, Levine RA, Tei C. Isolated annular dilation does not usually cause important functional mitral regurgitation: comparison between patients with lone atrial fibrillation and those with idiopathic or ischemic cardiomyopathy. J Am Coll Cardiol. 2002;39:1651-1656.

36. Otsuji Y, Gilon D, Jiang L, He S, Leavitt M, Roy MJ, Birmingham MJ, Levine RA. Restricted diastolic opening of the mitral leaflets in patients with left ventricular dysfunction: evidence for increased valve tethering. J Am Coll Cardiol. 1998;32:398-404.

37. Jouan J, Tapia M, Cook RC, Lansac E, Acar C. Ischemic mitral valve prolapse: mechanisms and implications for valve repair. Eur J Cardiothorac Surg. 2004;26:1112-1117.

38. Frater RWM, Cornellissen P, Sisto D. Mechanisms of ischemic mitral insufficiency and their surgical correction. In: Vetter HO, Hetzer R, Schmutzler H, eds. Ischemic Mitral Incompetence. New York, NY: Springer-Verlag; 1991:117-130.

39. Messas E, Guerrero JL, Handschumacher MD, Chow CM, Sullivan S, Schwammenthal E, Levine RA. Paradoxic decrease in ischemic mitral regurgitation with papillary muscle dysfunction: insights from three dimensional and contrast echocardiography with strain rate measurement. Circulation. 2001;104:1952-1957.

40. Khankirawatana B, Khankirawatana S, Mahrous H, Porter TR. Assessment of papillary muscle function using myocardial velocity gradient derived from tissue Doppler echocardiography. Am J Cardiol. 2004;94:45- 49.

41. Komeda M, Glasson JR, Bolger AF, Daughters GT II, Ingels NB Jr, Miller DC. Papillary muscleleft ventricular wall "complex." J Thorac Cardiovasc Surg. 1997;113:292-301.

42. Schwammenthal E, Chen C, Benning F, Block M, Breithardt G, Levine RA. Dynamics of mitral regurgitant flow and orifice area; physiologic application of the proximal flow convergence merhod: clinical data and experimental testing. Circulation. 1994;90:307-322.

43. Hung J, Otsuji Y, Handschumacher MD, Schwammenthal E, Levine RA.Mechanism of dynamic regurgitant orifice area variation in functional mitral regurgitation: physiologic insights from the proximal flow convergence technique. J Am Coll Cardiol. 1999;33:538 -545. 
44. Glasson JR, Komeda M, Daughters GT, Bolger AF, Karlsson MO, Foppiano LE, Hayase M, Oesterle SN, Ingels NB Jr, Miller DC. Early systolic mitral leaflet "loitering" during acute ischemic mitral regurgitation. J Thorac Cardiovasc Surg. 1998;116:193-205.

45. Schwammenthal E, Popescu AC, Popescu BA, Freimark D, Hod H, Eldar M, Feinberg MS. Mechanism of mitral regurgitation in inferior wall acute myocardial infarction. Am J Cardiol. 2002;90:306-309.

46. Miller DC. The three-dimensional geometry of the mitral valve: implications for surgical therapy. Proceedings of the 84th Annual Meeting of the American Association for Thoracic Surgery, Toronto, Ontario, 2004.

47. Condado JA, Vélez-Gimón. Catheter-based approach to mitral regurgitation. J Intervent Cardiol 2003;16:523-34.

48. Kodavatiganti $R$. Intraoperative assessment of the mitral valve by transoesophageal echocardiography: an overview.Ann Card Anaest 2002;5:27-34.

49. Gorman RC, Gorman JH III, Edmunds LH Jr. Ischemic mitral regurgitation. In: Cohn LH, Edmunds LH Jr, eds. Cardiac surgery in the adult. New York: McGraw-Hill, 2003:751-69.

50. Otsuji Y, Kumanohoso T, Yoshifuku S, et al. Isolated annular dilation does not usually cause important functional mitral regurgitation: comparison between patients with lone atrial fibrillation and those with idiopathic or ischemic cardiomyopathy. J Am Coll Cardiol 2002;39:1651-6.

51. Iung B. Management of ischaemic mitral regurgitation. Heart 2003;89:459-64.

52. Kumanohoso T, Otsuji Y, Yoshifuku S, et al. Mechanism of higher incidence of ischemic mitral regurgitation in patients with inferior myocardial infarction: quantitative analysis of left ventricular and mitral valve geometry in 103 patients with prior myocardial infarction. J Thorac Cardiovasc Surg 2003;125:135- 43.

53. Miller GE, Kerth WJ, Gerbode F. Experimental papillary muscle infarction. J Thorac Cardiovasc Surg 1968;56:611-6.

54. Messas E, Guerrero JL, Handschumacher MD, et al. Paradoxic decrease in ischemic mitral regurgitation with papillary muscle dysfunction: insights from three-dimensional and contrast echocardiography with strain rate measurement.Circulation 2001;104:1952-7.

55. Carabello B. The pathophysiology of mitral regurgitation. J Heart Valve Dis 2000;9:600-8.

56. Messas E, Guerro JL, Handschumacher MD, ct al. Chordal cutting. A new therapeutic approach for ischemic mitral regurgitation. Circulation 2001:104:1958-63.

57. Corin WJ, Monrad ES, Murakami T, et al. The relationship of afterload to ejection performance in chronic mitral regurgitation. Circulation 1987;76:59-67.

58. Jackson BM, Gorman JH III, Moainie SL, et al. Extension of borderzone myocardium in postinfarction dilated cardiomyopathy. J Am Coll Cardiol 2002;40:1160 -7.

59. Rumberger JA. Ventricular dilatation and remodeling after myocardial infarction. Mayo Clin Proc 1994;64:664 -74.

60. Kron IL, Green GR, Cope JT. Surgical relocation of the posterior papillary muscle in chronic ischemic mitral regurgitation. Ann Thorac Surg 2002;74:600-1. 
61. Lehmann KG, Francis CK, Dodge HT. Mitral regurgitation in early myocardial infarction. Incidence, clinical detection, and prognostic implications. TIMI Study Group. Annals of Internal Medicine 1992;117(1):10-7.

62. Bursi F, Enriquez-Sarano M, Nkomo VT, et al. Heart Failure and Death after Myocardial Infarction in the Community: the Emerging Role of Mitral Regurgitation. Circulation 2005; 11 (3): $254-6$.

63. Desjardins VA, Enriquez-Sarano M, Tajik AJ, Bailey KR, Seward JB. Intensity of murmurs correlates with severity of valvular regurgitation. American Journal of Medicine 1996; 100(2):149-56.

64. Enriquez-Sarano M, Rossi A, Seward JB, Bailey KR, Tajik AJ. Determinants of pulmonary hypertension in left ventricular dysfunction.Journal of the American College of Cardiology 1997; 29(1):153-9.

65. Hickey MS, Smith LR, Muhlbaier LH, et al. Current prognosis of ischemic mitral regurgitation. Implications for future management.Circulation 1988;78(3 Pe 2):151-9.

66. Spain MG, Smith MD, Grayburn PA, Harlamert EA, DeMaria AN. Quantitative assessment of mitral regurgitation by Doppler color flow imaging: angiographic and hemodynamic correlations. Journal of the American College of Cardiology 1989;13(3):585-90.

67. Roger VL, Jacobsen SJ, Weston SA, et al. Trends in the incidence and survival of patients with hospitalized myocardial infarction, Olmsted County, Minnesota, 1979 to 1994. Annals of Internal Medicine 2002;136(5): 341-8.

68. Zoghbi WA, Enriquez-Sarano M, Foster E, et al. Recommendations for evaluation of the severity of native valvular regurgitation with two-dimensional and Doppler echocardiography. Journal of the American Society of Echocardiography 2003; 16(7): 777-802.

69. Pierard LA, Lancellotti $P$. The role of ischemic mitral regurgitation in the pathogenesis of acute pulmonary edema. N Engl J Med 2004; 351(16): 1627-34.

70. Lancellotti P, Troisfontaines P, Toussaint AC, Pierard LA. Prognostic importance of exerciseinduced changes in mitral regurgitation in patients with chronic ischemic left ventricular dysfunction. Circulation 2003; 108(14): 1713-7.

71. Miller DC. Ischemic mitral regurgitation redux-to repair or to replace? J Thorac Cardiovasc Surg 2000;122:159-62.

72. 2004 Heart and stroke statistical update. available at: www.americanheart.org (accessed July 15, 2008).

73. Schwammenthal E, Chen C, Benning F, Block M, Breithardt G, Levine RA. Dynamics of mitral regurgitant flow and orifice area; physiologic application of the proximal flow convergence method: clinical data and experimental testing. Circulation. 1994;90:307-322.

74. Keren G, Katz S, Strom J, Sonnenblick EH, LeJemtel TH. Dynamic mitral regurgitation: an important determinant of the hemodynamic response to load alterations and inotropic therapy in severe heart failure. Circulation. 1989;80:306 -313.

75. Rodbard S, Williams F. The dynamics of mitral insufficiency. Am Hearr J. 1954;48:521-539.

76. Heinle SK, Tice FD, Kisslo J. Effect of dobutamine stress echocardiographyon mitral regurgitation. J Am Coll Cardiol. 1995;25:122-127. 
77. Keren G, Bier A, Strom JA, Laniado S, Sonnenblick EH, LeJemtel 'TH. Dynamics of mitral regurgitation during nitroglycerin therapy: a Doppler echocardiographic study. Am Heart J. 1986;112:517-525.

78. Stevenson LW, Bellil D, Grover-McKay M, Brunken RC, Schwaiger M, Tillisch JH, Schelbert HR. Effects of afterload reduction (diuretics and vasodilators) on left ventricular volume and mitral regurgitation in severe congestive heart failure secondary to ischemic or idiopathic dilated cardinmyopathy. Am J Cardiol. 1987;60:654-658.

79. Hamilton MA, Stevenson LW, Child JS, Moriguchi JD, Walden J, Woo M. Sustained reduction in valvular regurgitation and atrial volumes with tailored vasodilator therapy in advanced congestive heart failure secondary to dilated (ischemic or idiopathic) cardiomyopathy. Am J Cardiol. 1991;67:259-263.

80. Rosario LB, Stevenson LW, Solomon SD, Lee RT, Reimold SC. The mechanism of decrease in dynamic mitral regurgitation during heart failure treatment: importance of reduction in the regurgitant orifice size. J Am Coll Cardiol. 1998;32:819-1824.

81. Levine AB, Muller $\mathrm{C}$, Levine TB. Effects of high-dose lisinoprilisosorbide dinitrate on severe mitral regurgitation and heart failure remodeling. Am J Cardiol. 1998;82:1299-1301.

82. Kizilbash AM, Willetr DL, Brickner ME, Heinle SK, Grayburn PA. Effects of afterload reduction on vena contracta width in mitral regurgitation. J Am Coll Cardiol. 1998;32:427-431.

83. Pfeffer MA, Lamas GA, Vaughan DE, Parisi AF, Braunwald E. Effect of captopril on progressive ventricular dilatation after anterior myocardial nfarction. N Engl J Med. 1988;319:80-86.

84. Levine and Schwammenthal Ischemic Mitral Regurgitation Downloaded from circ.ahajournals. org by on July 24,2008

85. St John Sutton M, Pfeffer MA, Moye L, Plappert T, Rouleau JL, LamasG, Rouleau J, Parker JO, Arnold MO, Sussex B, Braunwald E. Cardiovascular death and left ventricular remodeling two years after myocardial infarction: baseline predictors and impact of long-term use of captopril: information from the Survival and Ventricular Enlargement (SAVE) trial. Circulation. 1997;96:3294-3299.

86. Greenberg B, Quinones MA, Koilpillai C, Limacher M, Shindler D, Benedict C, Shelton B. Effects of long-term enalapril therapy on cardiac structure and function in patients with left ventricular dysfunction: results of the SOLVD echocardiography substudy. Circulation. 1995; 91:2573-2581.

87. Konstam MA, Kronenberg MW, Rousseau MF, Udelson JE, Melin J, Stewart D, Dolan N, Edens TR, Ahn S, Kinan D; SOLVD (Studies of Left Ventricular Dysfunction) Investigators. Effects of the angiotensin converting enzyme inhibitor enalapril on the long-term progression of left ventricular dilatation in patients with asymptomatic systolic dysfunction. Circulation. 1993;88:2277-2283.

88. Carabello BA, Nakano K, Corin W, Biederman R, Spann JF Jr. Left ventricular function in experimental volume overload hypertrophy. Am J Physiol. 1989;256:H974-H981.

89. Tsutsui H, Spinale FG, Nagatsu M, Schmid PG, Ishihara K, DeFreyte G, Cooper G IV, Carabello BA. Effects of chronic beta-adrenergic blockade on the left ventricular and cardiocyte abnormalities of chronic canine mitral regurgitation. J Clin Invest. 1994 93:2639-2648. 
90. Lowes BD, Gill EA, Abraham WT, Larrain JR, Robertson AD, Brisrow MR, Gilbert EM. Effects of carvedilol on left ventricular mass, chamber geometry, and mitral regurgitation in chronic heart failure. Am J Cardiol. 1999;83:1201-1205.

91. Capomolla S, Febo O, Gnemmi M, Riccardi G, Opasich C, Caporotondi A, Mortara A, Pinna GD, Cobelli F. Beta-blockade therapy in chronic heart failure: diastolic function and mitral regurgitation improvement by carvedilol. Am Heart J. 2000;139:596-608.

92. Remme WJ, Riegger G, Hildebrandt P, Komajda M, Jaarsma W, Bobbio M, Soler-Soler J, Scherhag A, Lutiger B, Ryden L. The benefits of early combination treatment of carvedilol and an ACEinhibitor in mild heart failure and left ventricular systolic dysfunction: the carvedilol and ACE inhibitor remodeling mild heart failure evaluation trial (CARMEN). Cardiovasc Drugs Ther. 2004;18:57-66.

93. Doughty RN, Whalley GA, Walsh HA, Gamble GD, Lopez-Sendon J, Sharpe N; CAPRICORN Echo Substudy Investigators. Effects of carvedilol on left ventricular remodeling after acute myocardial infarction: the CAPRICORN Echo Substudy. Circulation. 2004;109:201-206.

94. Tei C, Horikiri Y, Park JC, Jeong JW, Chang KS, Toyama Y, Tanaka N. Acute hemodynamic improvement by thermal vasodilation in congestive heart failure. Circulation. 1995;91:25822590.

95. Mukherjee R, Brinsa TA, Dowdy KB, Scott AA, Baskin JM, Deschamps AM, Lowry AS, Escobar GP, Lucas DG, Yarbrough WM, Zile MR, Spinale FG. Myocardial infarct expansion and matrix metalloproteinase inhibition. Circulation. 2003;107:618-625.

96. St John Sutton MG, Plappert T, Abraham WT, Smith AL, DeLurgio DB, Leon AR, Loh E, Kocovic DZ, Fisher WG, Ellestad M, Messenger J, Kruger K, Hilpisch KE, Hill MR; Multicenter InSync Randomized Clinical Evaluation (MIRACLE) Study Group. Effect of cardiac resynchronization therapy on left ventricular size and function in chronic heart failure. Circulation. 2003;107:19851990.

97. Kanzaki H, Bazaz R, Schwartzman D, Dohi K, Sade LE, Gorcsan J III. A mechanism for immediate reduction in mitral regurgitation after cardiac resynchronization therapy: insights from mechanical activation strain mapping. J Am Coll Cardiol. 2004;44:1619-1625.

98. Breithardt OA, Sinha AM, Schwammenthal E, Bidaoui N, Markus KU, Franke A, Stellbrink C. Acute effects of cardiac resynchronization therapy on functional mitral regurgitation in advanced systolic heart failure. J Am Coll Cardiol. 2003;41:765-770.

99. Condado JA, Vélez-Gimón. Catheter-based approach to mitral regurgitation. J Intervent Cardiol 2003;16:523-34.

100. Canadian Cardiovascular Consensus 2004: surgical management of valvular heart disease. Can J Cardiol 2004;20:33E-49E.

101. Mallidi HR, Pelletier MP, Lamb J, et al. Late outcomes in patients with uncorrected mild to moderate mitral regurgitation at the time of isolated coronary artery bypass grafting. J Thorac Cardiovasc Surg 2004;127:636-44.

102. Aklog L, Filsoufi F, Flores KQ, et al. Does coronary artery bypass grafting alone correct moderate ischemic mitral regurgitation?Circulation 2001;12(Suppl 1):68 -75. 
103. Lam BK, Gillinov AM, Blackstone EH, et al. Importance of moderate ischemic mitral regurgitation. Ann Thorac Surg 2005;79:462-70.

104. Wong DR, Agnihotri AK, Hung JW, et al. Long-term survival after surgical revascularization for moderate ischemic mitral regurgitation. Ann Thorac Surg 2005;80:570-8.

105. David TE, Uden DE, Strauss $H$. The importance of the mitral apparatus in left ventricular function after correction of mitral regurgitation. Circulation 1983;68(Suppl 2):76-82.

106. David TE, Armstrong S, Sun Z. Left ventricular function after mitral valve surgery. J Heart Valve Dis 1995; 4 (Suppl): $175-80$.

107. Calafiore AM, Di Mauro M, Gallina S, et al. Mitral valve surgery for chronic ischemic mitral regurgitation. Ann Thorac Surg 2004;77:1989-97.

108. Gillinov AM, Wierup PN, Blackstone EH, et al. Is repair preferable for ischemic mitral regurgitation? J Thorac Cardiovasc Surg 2001;122:1125- 41.

109. Grossi EA, Goldberg JD, LaPietra A, et al. Ischemic mitral valve reconstruction and replacement: comparison of longterm survival and complications. J Thorac Cardiovasc Surg 2001;122:110724.

110. Al-Radi OO, Austin PC, Tu JV, David TE, Yau TM. Mitral repair versus replacement for ischemic mitral regurgitation. Ann Thorac Surg 2005;79:1260-7.

111. Bolling SF, Pagani FD, Deeb GM, Bach DS. Intermediate term outcome of mitral reconstruction in cardiomyopathy. J Thorac Cardiovasc Surg 1998;115:381-6.

112. McCarthy PM. Does the intertrigonal distance dilate? Never say never. J Thorac Cardiovasc Surg 2002;124:1078-9.

113. McGee EC, Gillinov AM, Blackstone EH, et al. Recurrent mitral regurgitation after annuloplasty for functional ischemic mitral regurgitation. J Thorac Cardiovasc Surg 2004;128:916-24.

114. Bolling SF, Deeb GM, Bach DS. Mitral valve reconstruction in elderly, ischemic patients. Chest 1996;109:35- 40.

115. Tahta SA, Oury JH, Maxwell JM, Hiro SP, Duran CM. Outcome after mitral valve repair for functional ischemic mitral regurgitation. J Heart Valve Dis 2002;11:11-8.

116. von Oppell UO, Stemmet F, Brink J, Commerford PJ, Heijke SA. Ischemic mitral valve repair surgery. J Heart Valve Dis 2000;9:64-74.

117. Hung J, Papakostas L, Tahta SA, et al. Mechanism of recurrent ischemic mitral regurgitation after annuloplasty: continued LV remodeling as a moving target. Circulation 2004; 110(Suppl 2):85-90.

118. Votra E, Maisano F, Bolling SF, Alfieri O, Montevecchi FM, Redaelli A. The Geoform diseasespecific annuloplasty system: a finite element study. Ann Thorac Surg. 2007;84(1):92-101.

119. Salgo IS, Gorman JH, Gorman RC, et al. Effect of Annular Shape on Leaflet Curvature in Reducing Mitral Leaflet Stress. Circulation. 2002; 106:711-717.

120. Jimenez JH, Yoganathan AP, et al. Effects of a saddle shaped annulus on mitral valve function and chordal force distribution: An in vitro Study. Annals of Biomedical Engineering. Vol. 31, pp. $1171-1181,2003$. 
121. Rodriguez F, Langer F, Harrington KB, et al. Importance of mitral valve second-order chordae for left ventricular geometry, wall thickening mechanics, and global systolic function. Circulation 2004;110(Suppl 2):115-22.

122. Yamamoto H, Iguro Y, Sakata R, Arata K, Yotsumoto G. Effectively treating ischemic mitral regurgitation with chordal cutting in combination with ring annuloplasty and left ventricular reshaping approach. J Thorac Cardiovasc Surg 2005;130:589 -90.

123. Borger MA, Alam A, Murphy PM, Doenst T, David TE. Chronic Ischemic Mitral Regurgitation: Repair, Replace or Rethink?. Ann Thorac Surg 2006; 81: 1153-61.

124. Alfieri O, Maisano F, De Bonis $M$, et al. The double orifice technique in mitral valve repair: a simple solution for complex problems. J Thorac Cardiovasc Surg 2001:122:674-81.

125. Maisano F, Caldarola A, Blasio A, De Bonis M, La Canna G, Alfieri O. Midterm results of edgeto-edge mitral valve repair without annuloplasty. J Thorac Cardiovasc Surg 2003; 126:1987-97.

126. Bhudia SK, McCarthy MM, Smedira NG, et al. Edge-ro-edge (Alifieri) mitral repair. Ann Thorac Surg 2004;77:1598-606.

127. Ramadan R, Al-Attar N, Mohammadi S, et al. Left ventricular infarct plication restores mitral function in chronic ischemic mitral regurgitation. J Thorac Cardiovasc Surg 2005;129:440-2.

128. Menicanti L, Di Donato M, Frigoiola A, et al. Ischemic mitral regurgitation: intraventricular papillary muscle imbrication without mitral ring during left ventricular restoration. J Thorac Cardiovasc Surg 2002;123:1041-50.

129. Sabatier M, Di Donato M, Montiglio F, Toso A, Maioli M. Efficacy of endoventricular patch plasty in large postinfarction akinetic scar and severe left ventricular dysfunction: comparison with a series of large dyskinetic scars. J Thorac Cardiovasc Surg 1998;116:50-9.

130. Hvass U, Tapia M, Baron F, Pouzet B, Shafy A. Papillary muscle sling: a new functional approach to mitral repair in patients with ischemic left ventricular dysfunction and functional mitral regurgitation. Ann Thorac Surg 2003;75: 809-11.

131. Fundaro P, Pocar M, Moneta A, et al. Posterior mitral valve restoration for ischemic regurgitation. Ann Thorac Surg 2004;77:729-30.

132. Fann JI, St Goar FG, Komtebedde J, et al. Bearing heart catheter-based edge-to-edge mitral valve procedure in a porcine model: efficacy and healing response. Circulation 2004;110:988-9.

133. Daimon $M$, Shiota $T$, Gillinov AM, et al. Percutaneous mitral valve repair for chronic ischemic mitral regurgitation: a real-time three-dimensional echocardiographic study in an ovine model. Circulation 2005;111:2183-9.

134. Maniu CV, Patel DG, Reuter DG, et al. Acute and chronic reduction of functional mitral regurgitation in experimental heart failure by percutaneous mirral annuloplasty. J Am Coll Cardiol 2004;44:1652-61.

135. Singh SK, Borger MA. Percutaneous valve replacement: fact or fiction? Can J Cardiol 2005;21:82932.

136. Tibayan F, Rodriguez F, Langer F, et al. Does septal-lateral annular cinching work for chronic ischemic mitral regurgitation? J Thorac Cardiovasc Surg 2004;127:654-63.

137. Timek TA, Dagum P, Glasson JR, et al. Restricted posterior leaflet motion after mitral ring annuloplasty. Ann Thorac Surg 1999;68:2100-6. 
138. Fukamachi $K$, Inoue M, Popovic' ZB, et al. Off-pump mitral valve repair using the Coapsys device: a pilot study in a pacing-induced mitral regurgitation model. Ann Thorac Surg 2004;77:688-93.

139. Konertz W, Rombeck B, Horz H. Short-term safety of the Acorn cardiac support device in patients with advanced heart failure. J Am Coll Cardiol 2000;35:182A.

140. Livi U, Alfieri O, Vitali E, et al. One-year clinical experience with the Acorn CorCap cardiac support device: results of a limited market release safety study in Italy and Sweden. Ital Heart J. $2005 \mathrm{Jan} ; 6(1): 59-65$ 


\section{2}

Five-Year Echocardiographic Results of Combined Undersized Mitral Ring Annuloplasty and Coronary Artery Bypass

Grafting for Chronic Ischemic Mitral

Regurgitation

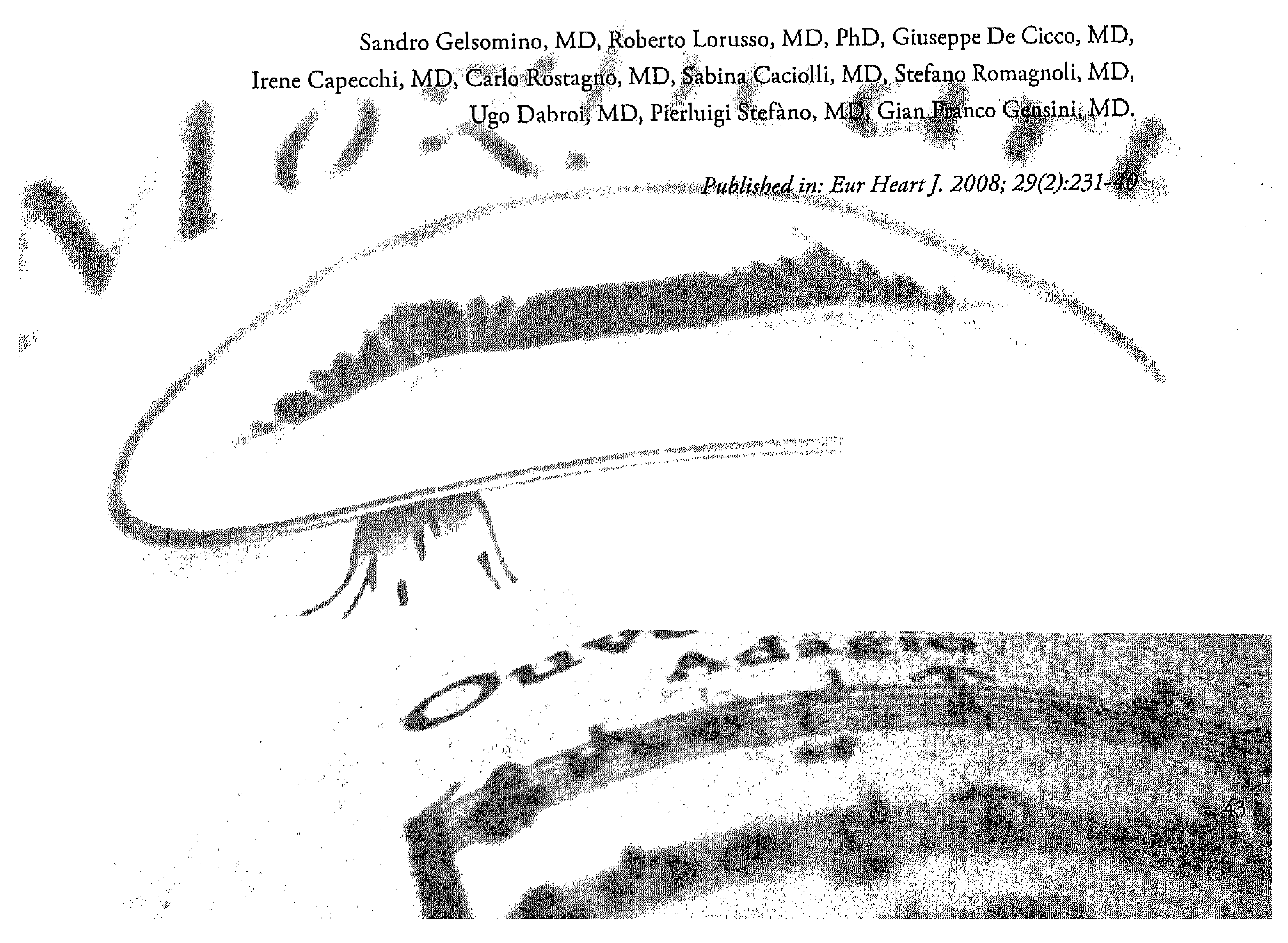




\section{ABSTRACT}

Aims. We present 5-year echocardiographic results of combined undersizing mitral ring annuloplasty (UMRA) and coronary arrery bypass grafting (CABG) in ischemic mitral regurgitation (CIMR).

Methods and Results. Two-hundred-fifty-one patients (age $68.4 \pm 8.1,62.5 \%$ male) undergoing combined CABG and UMRA in our Institution (Cardiac Surgery, Careggi Hospital, Florence, Italy) between September 2001 and March 2007, were prospectively enrolled in the study. Median follow up was 32.9 months (interquartile range [IQR] 17.5-51.6). Fourteen patients with significant residual mitral regurgitation (MR) needing immediate intraoperative revision $(n=3)$ or at discharge $(n=11)$ werc excluded from the study. Serial echocardiograms were performed in 220 survivors at baseline, discharge and annually thereafter. Additionally, 17 patients died ( 2 early and 15 late deaths) and were also excluded from the study. MR remained stable at 1 year and re-increased at 3 years $(\mathrm{p}<0.001)$ and 5 years $(\mathrm{p}<0.001)$. Five-year actuarial survival was $83.2 \pm 4.4$. Five-year freedom from reoperation for failed repair was $78.2 \pm 4.9 \%$. Mean systolic and diastolic diameters decreased significantly at discharge ( $p=0.001$ and $p=0.01$, respectively) and at carly follow up ( $p=0.004$ and $p=0.02)$ but was raised at 3 years $(p<0.001)$ and 5 years $(p<0.001)$. Systolic and diastolic sphericity indexes improved at discharge $(p<0.001)$, remained stable at 1-year but they re-increased at 3 -year control $(p=0.006$ and $\mathrm{p}=0.03$. respectively) with a late raise exceeding the preoperative value $(\mathrm{p}<0.001)$. Left ventricular reverse remodeling was observed in $44.2 \%$ of the study population with $10.3 \%$ of patients showing further left ventricular dilatation. At multivariable model, end-systolic volume $\geq 145 \mathrm{ml}$, systolic sphericity Index $\geq 0.7$, Myocardial performance Index $\geq 0.9$ and wall motion score Index $\geq 1.5$ were predictors of recurrent MR.

Conclusions Our findings emphasize the need for improved repair technique and better patient selection to identify patients with anticipated repair failure who could benefit more from valve replacement or other procedure directly addressing ventricular tethering. 


\section{INTRODUCTION}

Despite of improvements in mitral reparative techniques in the recent years ${ }^{1}$, chronic ischemic mitral regurgitation (CIMR) still remains a challenging surgical problem. Combined coronary artery bypass grafting (CABG) and annular reduction with an annuloplasty ring (undersized mitral ring annuloplasty, UMRA) is the most common surgical technique employed to relieve ischemic regurgitation ${ }^{2}$. Nonetheless, conflicting data are reported in the literature about long-term effectiveness of this approach ${ }^{3-7}$.

In the present study, we present 5 -year echocardiographic results after combined UMRA and CABG in terms of recurrence of mitral regurgitation (MR), global and local LV remodeling and left ventricular reverse remodeling (LVRR). We also explore potential useful predictors of recurrence of MR following combined undersized annuloplasty and coronary artery bypass.

\section{METHODS}

\section{Patients population}

Chronic ischemic mitral regurgitation was defined as the combination of mildto-severe MR with 1) Prior myocardial infarction (MI) $>16$ days; 2) seventy-five percent or greater stenosis of at least one coronary vessel; 3) a corresponding regional wall motion abnormality; 4) type IIIb leaflet dysfunction following Carpentier's classification ${ }^{8}$ with or without annular dilatation.

Exclusion criteria were: 1) degenerative or other non-ischemic etiology; 2) ischemic isolated type I or type II dysfunction ${ }^{8}$; 3 ) additional mitral valve repair procedures; 4) other valvular or congenital heart diseases.; 5) previous cardiac surgery/percutaneous transluminal coronary angioplasty; 6) atrial fibrillation or sinus rhythm with heart rate at rest $>100 \mathrm{bts} / \mathrm{min}$.

Two-hundred-fifty-one patients undergoing combined CABG and UMRA in our Institution (Cardiac Surgery, Careggi Hospital, Florence, Italy) between September 2001 and March 2007, were prospectively enrolled in the study (Table 1). Clinical follow up information was obtained from all survivors through outpatient visits and phone calls and was 100\% complete. Median follow up was 32.9 months (interquartile range $[\mathrm{YQR}]$ 17.5-51.6).

Following the World Medical Association guidelines concerning ethical principles for medical research involving human subjects ${ }^{9}$, the study was approved by Institutional Ethics Board. Furthermore, all patients gave their informed consent. 
Table 1. Perioperative data $(n=251)$.

\begin{tabular}{|c|c|}
\hline Age (years) & $68.4 \pm 8.1$ \\
\hline Gender $\mathrm{M} / \mathrm{F}$ & $155 / 93(62.5 / 37.5)$ \\
\hline NYHA class & $3.2 \pm 0.6$ \\
\hline CCS angina class & $3.09 \pm 0.4$ \\
\hline \multicolumn{2}{|l|}{ Euroscore } \\
\hline Additive & $8.0 \pm 4.3$ \\
\hline Logistic & $13.6 \pm 6.1$ \\
\hline Hypertension & $92(36.6)$ \\
\hline Diabetes & $71(28.6)$ \\
\hline COPD & $32(12.7)$ \\
\hline Chronic renal disease & $26(10.3)$ \\
\hline Cerebral vascular disease & $15(6,0)$ \\
\hline Peripheral vascular disease & $11(4.3)$ \\
\hline Familiar history & $101(40.2)$ \\
\hline \multicolumn{2}{|l|}{ Myocardial infarction } \\
\hline$>3$ months & $197(78.4)$ \\
\hline$\leq 3$ months & $54(21.6)$ \\
\hline Inferior/Posterior & $122(48.6)$ \\
\hline Anterior/Septal & $22(8.8)$ \\
\hline Lateral & $49(19.5)$ \\
\hline Combined & $58(23.1)$ \\
\hline Diseased coronary vessels & $2.4 \pm 0.5$ \\
\hline 1 & $15(6.0)$ \\
\hline 2 & $107(42,6)$ \\
\hline$\geq 3$ & $129(51.4)$ \\
\hline Left Main & $38(15.1)$ \\
\hline \multicolumn{2}{|l|}{ Medications } \\
\hline Angiotensin-converting enzyme inhibitors & $197(78.4)$ \\
\hline B-adrenergic blockers & $132(52.6)$ \\
\hline Long-acting nitrates & $109(43.4)$ \\
\hline Diuretics & $185(73.7)$ \\
\hline Digitalis & $50(19.9)$ \\
\hline Calcium antagonists & $33(13.1)$ \\
\hline Preoperative IABP & $11(4.3)$ \\
\hline \multicolumn{2}{|l|}{ Surgery } \\
\hline \multicolumn{2}{|l|}{ Cardioplegia } \\
\hline Antegrade & $218(86.8)$ \\
\hline Antegrade and Rerrograde & $33(13.2)$ \\
\hline $\mathrm{CPB}$ time $(\min )$ & $123 \pm 31$ \\
\hline CCL time (min) & $92 \pm 18$ \\
\hline
\end{tabular}




\begin{tabular}{|c|c|}
\hline \multicolumn{2}{|l|}{ Surgical Approach } \\
\hline Left atriotomy & $207(82.4)$ \\
\hline Transseptal & $25(10.0)$ \\
\hline Transseptal extended to LA roof & $19(7.6)$ \\
\hline \multicolumn{2}{|l|}{ Ring } \\
\hline${ }^{*}$ Carpentier Classic & $133(52.9)$ \\
\hline *Physio & $118(47.1)$ \\
\hline Ring size $(\mathrm{mm})$ & $27.4 \pm 1.5$ \\
\hline $24 \mathrm{~mm}$ & $10(4.1)$ \\
\hline $26 \mathrm{~mm}$ & $92(36.6)$ \\
\hline $28 \mathrm{~mm}$ & $112(44.6)$ \\
\hline $30 \mathrm{~mm}$ & $37(14.7)$ \\
\hline \multicolumn{2}{|l|}{$\mathrm{CABG}$} \\
\hline Anastomoses/patient & $2.2 \pm 1.1$ \\
\hline Arterial graft/patient & $1.07 \pm 0.1$ \\
\hline \multicolumn{2}{|c|}{$\begin{array}{l}\text { Continuous variables are presented as mean } \pm \text { standard deviation; Discrete variables are presented as } \\
\text { percentage. Abbreviations: } M / F=M a l e / F e m a l e ; ~ N Y H A=N e w \text { York Heart Association; } C C S=\text { Canadian } \\
\text { Cardiovascular Society; } C O P D=C h r o n i c \text { obstructive pulmonary disease; } I A B P=I \text { Intaaortic balloon } \\
\text { pump; } C P B=\text { Cardiopulmonary bypass; } C C L=\text { (Aortic) Cross-clamp; } M R=\text { Mitral regurgitation; } \\
C A B G=\text { Coronary artery bypass grafting. }{ }^{*} \text { Edwards LifeSciences, Irvine, } C A \text {. }\end{array}$} \\
\hline
\end{tabular}

\section{SURGERY}

All patients underwent associated coronary artery bypass grafting. For the purpose of this study, complete revascularization was accomplished when, at least one graft was placed distal to an approximately $50 \%$ diameter narrowing in each of the three major vascular system in which arterial narrowing of this severity was noted in a vessel $\geq 1.5 \mathrm{~mm}$ of diameter. It was not considered necessary to bypass all obstructed diagonal branches of the anterior descending or marginal branches of the circumflex coronary arteries for a classification of complete revascularization. Following this definition $100 \%$ patients underwent complete revascularization.

The ring size was determined by standard measurements of the inter-trigonal distance and anterior leaflet height. A downsizing by two ring sizes was performed in all patients. After cardiopulmonary bypass (CPB), a transesophageal echocardiography (TEE) was performed to assess residual $M R$ : leaflet coaptation $\geq 0.5 \mathrm{~cm}, M R$ $\leq 1$ and systolic MV area $>2 \mathrm{~cm}^{2}$ was assessed as successful repair. Three patients needed immediate intraoperative revision for failed repair: all underwent mitral valve replacement and were excluded from the study. 


\section{ECHOCARDIOGRAPHIC MEASUREMENTS AND CALCULATIONS}

The clinical echocardiographic evaluation was as follows. A transthoracic echocardiogram (TTE) and a TEE were performed within 5 days before surgery and serial TTE were performed at discharge and annually thereafter. Eleven patients (4.4\%) with residual MR defined as the insufficiency $\geq 2+$ following valve repair documented at discharge, were excluded from the study. Five-year echocardiograms were available from 61 patients (27.7\%). This patient dropout was independent of the disease status and totally due to the different number of patients reaching different study intervals (1-year, 187 patients [85\% ]; 3-year 122 [55.4\% ]. Echocardiographic studies were carried out on Acuson Sequoia imaging device equipped with a $3.5-\mathrm{MHz}$ ultrasound transducer (Acuson Corporation, Mountain View, CA). Standard parasternal and apical views (long axis, short-axis, 2-4-5-chamber views) were employed. The reliability of echocardiographic measurements was assessed by calculating between-observer (IC and CR) interval of agreements of main direct measures used in this study in a different group of 20 subjects $(10 \mathrm{MR})^{11}$.

\section{Mitral Regurgitation}

The following quantitative measurements were simultaneously employed to grade the severity of $M R$ and final results were averages of measured values ${ }^{12-14}$ : (1) Quantitative Doppler. (2) Proximal Isovelocity Surface Area (PISA). For each measurement a minimum of three cardiac cycles was averaged. The respective thresholds for mild, moderate and severe MR were $<30,30-59$ and $\geq 60 \mathrm{ml} /$ beat for regurgitant volume (RV), $<30,30-49$ and $\geq 50 \%$ for regurgitant fraction (RF), $<20,20-39$ and $\geq 40 \mathrm{~mm}^{2}$ for effective regurgitant orifice (ERO), respectively. In patients with no or trivial MR by color Doppler, RV and RF were used as calculated, and ERO was assumed as null.

The tenting area (TA) was measured by the area enclosed between the annular plane and mitral leaflets from the parasternal long-axis view at mid-systole. The distance between leaflet coaptation and the mitral annulus plane at early and end systole measured displacement of mitral coaptation toward the LV apex ${ }^{15,16}$. Mitral annular areas were obtained from mitral annular dimensions in apical long-axis, 4-chamber, and 2-chamber views, using an ellipsoid assumption ${ }^{17}$.

Recurrent MR was the insufficiency $\geq 2+$ at follow up appointments in patients with no/trivial MR at discharge.

\section{Global LV Remodeling}

LV volumes and left ventricular ejection fraction (LVEF) were assessed by the bi-apical Simpson disk method ${ }^{18}$. According to Stellbrink et coworkers ${ }^{19}$ a decrease 
in LV end systolic volume $>15 \%$ from baseline value was considered LVRR. The myocardial performance index (MPI) was measured using the method described by Tei et al. ${ }^{20}$. Sphericity indexes were obtained at end diastole and end systole $\left(\mathrm{SI}_{\mathrm{D}}\right.$ and $\mathrm{SI}_{S}$, respectively) as the volume of the left ventricle divided by the volume of a sphere with a diameter equal to the longest axis of the left ventricle measured in the apical view ${ }^{21}$. The wall motion score index (WMSI) was calculated according to a 17-segment model $^{22}$

\section{LOCAL LV REMODELING}

The displacement of papillary muscles (PMs) was quantified as distances from well-defined anatomic landmarks at early and end systole ${ }^{15}$. Lateral and posterior displacements of anterior papillary muscles (APM) and posterior papillary muscles (PPM) were measured as distances from these fixed references. Separation between papillary muscles was directly measured (from body to body of PMs). In the longaxis view the apical displacement of the PPM was measured as the distance between the papillary muscle head and the fixed inter-valvular fibrosa (annular-papillary distance). The WMSI of the basal and mid-posterior and inferior segments for the PPM and of basal and mid-lateral and anterior segments for the APM were calculated $^{23}$.

\section{Statistical Analysis}

The sample size was determined by GraphPad StatMate release 2.00 (GraphPad Prism Software, Inc, San Diego, CA), on the basis of preliminary data obtained by echocardiography and was determined on the basis of the following assumptions: Type I error of 0.05 (two-sided), power of $80 \%$, difference in end systolic volume between patients with or without recurrent mitral regurgitation of 0.78 , standard deviation of 2.4. The calculated study population was 300 . However 256 patients were recruited to allow for possible analytic problems while processing the or other eventualities potentially leading to patient attrition. Continuous variables are presented as mean and standard deviation (SD), categorical variables as $\mathrm{n} / \mathrm{N}(\%)$, odds ratios as OR, and pertinent $95 \%$ confidence intervals as $\mathrm{Cl}$. The bivariate association between the independent variables and recurrent mitral regurgitation was assessed by the Student's t-test, Mann Whitney U test, $\chi^{2}$ test and Fisher exact test where appropriate Echocardiographic variables over time were analyzed by means of ANOVA repeated measures followed by Tukey post-hoc test and Friedman test, where appropriate to detect differences at different study points (discharge vs. baseline; 1-year vs. discharge; 3-year vs.1-year and 5-year vs. 3-year).

Multivariable logistic regression analysis by means of a backward stepwise algorithm (cut-off for entry 0.05 , for removal 0.10 ) was performed to select independent 
predictors of recurrent regurgitation. Model assumptions (linearity and additivity assumptions) were checked by piecewise cubic polynomials (spiline functions) and pooled interaction test ${ }^{24}$, respectively, and found to be satisfied. Goodness of fit of the final logistic regression models was assessed with the Hosmer-Lemeshow statistic $^{25}$ and predictive accuracy was assessed by the concordance (c) index ${ }^{24}$. Internal validation of predictors generated by multivariable logistic regression was performed by means of bootstrapping techniques, with 1000 cycles and generation of OR and bias corrected $95 \% \mathrm{CI}^{24}$

Optimal cutoff values were determined as the rounding cut-off that gives the maximum sum of sensitivity and specificity. This value should be the shoulder at the top left of the ROC (receiver operating characteristic curve). Bootstrapping techniques were employed to validate the results. Cumulative probability for death and reoperation were estimated by use of the Kaplan-Meier method.

SPSS 12.0 (SPSS, Chicago, IL, USA) and Stats Direct 2.5.7 (StatsDirect, Sale, UK) were used for these calculations. Significance for hypothesis testing was set at the 0.05 two-tailed level.

\section{RESULTS}

\section{Overall Survival}

Early (30-day) mortality was $0.8 \%(n=2$, respiratory failure and low-output syndrome). During the follow-up 15 patients died: six of cardiac causes (heart failure [n=3;36, 45, and 54 months], myocardial infarction [n=2; 31 and 42 months] and arrhythmia [n=1;59 months]), eight of non-cardiac causes (multiorgan failure $[\mathrm{n}=1$; 6 months], respiratory failure [ $\mathrm{n}=1 ; 14$ months], infection [ $\mathrm{n}=2 ; 3,8$ months], stroke $[\mathrm{n}=2 ; 12,43$ months] and malignancy [ $\mathrm{n}=2 ; 55,59$ months]) and 1 of sudden, unexplained death (22 months). Five-year actuarial survival was $83.2 \pm 4.4$ (Figure 1).

\section{Residual and Recurrent Mr}

Among 220 patients surviving CABG and reductive annuloplasty (Figure 2) mean MR remained stable at 1 year and re-increased at 3-year $(\mathrm{p}<0.001)$ and 5 -year studies(p<0.001, Table 2). At 5-year echocardiography 17 patients $(27.8 \%)$ had no/ trivial recurrent MR, 17 (27.8\%) had MR 2+, 19 (31.1\%) 3+ and 8 (13.1\%) 4+ (Figure 3). Seventeen patients underwent reoperation for failed repair due to recurrent MR: four $(3.7 \%)$ had a re-MV repair and $13(12.1 \%)$ underwent mitral valve replacement. Freedom from reoperation for failed repair was $78.2 \pm 4.9 \%$ (Figure 4). 
Survival

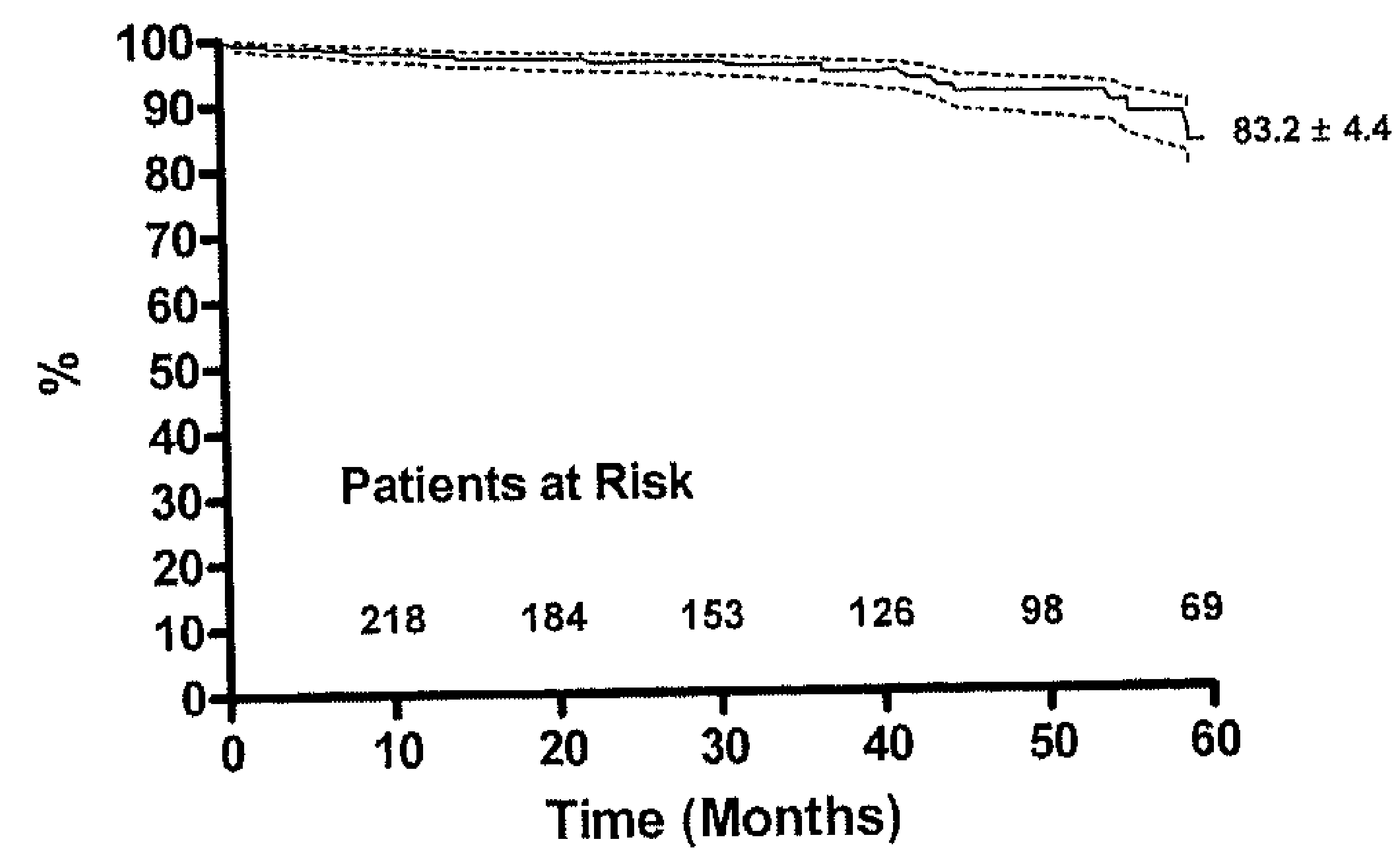

Figure 1. Five-year survival and $95 \%$ Confidence interval (dotted lines) following undersized mitral ring annuloplasty and coronary artery bypass grafting in patients with ischemic mitral regurgitation.

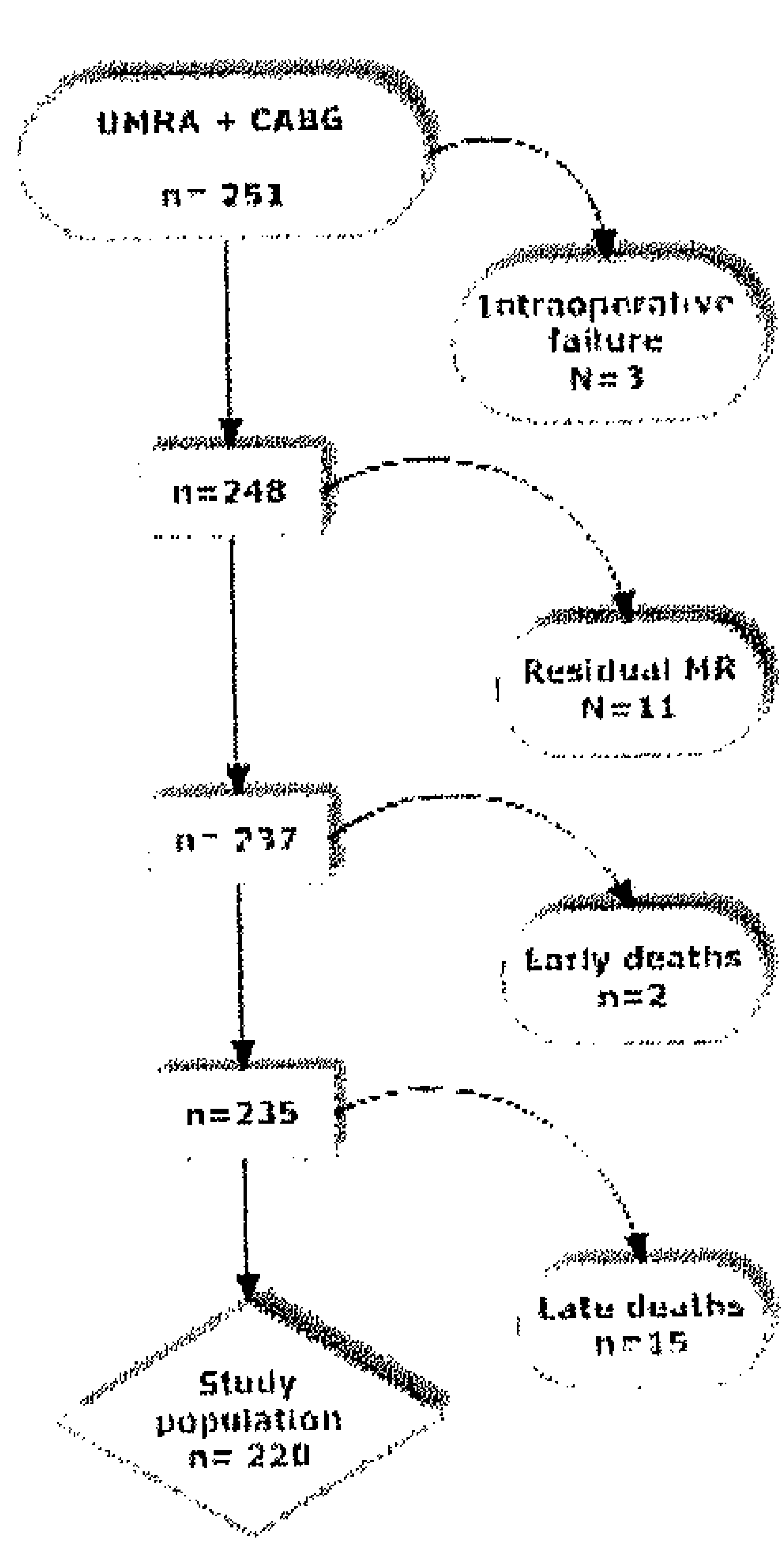

Figure 2. Patient selection. Abbreviations: $U M R A=$ Undersized mitral ring annuloplasty; $\mathrm{CABG}=$ Coronary artery bypass grafting; MR=Mitral regurgitation. See text for definitions.

\section{Global LV Remodeling and LV}

\author{
REVERSE REMODELING
}

Ventricular diameters and left atrial dimensions reduced significantly at discharge $(p<0.001$, $\mathrm{p}=0.005, \mathrm{p}=0.002$, respectively), showing no further significant change at subsequent controls (Table 3). Mean ESV and EDV decreased significantly at discharge $(\mathrm{p}=0.001$ and $\mathrm{p}=0.01$, respectively) and at early follow up ( $p=0.004$ and $p=0.02)$ but re-increased at 3-year $(p<0.001)$ and 5 -year $(p<0.001)$ studies. Furthermore, ejection fraction and MPI did not show significant changes over time. Systolic and diastolic sphericity indexes improved at discharge $(p<0.001)$, remained stable at 1-year $(\mathrm{p}=\mathrm{ns})$ but they were raised at 3 -year control $\left(p=0.006\right.$ and $p=0.03$ for $\mathrm{SI}_{S}$ and $\mathrm{SI}_{\mathrm{D}_{\mathrm{s}}}$ respectively) with a late increase exceeding the preoperative value $(p<0.001)$. Finally, WMSI reduced significantly at discharge, remained constant at 1 year, but re-increased at 3 -year $(p=0.02)$ and 5 -year $(p<0.001)$ echocardiograms. Sixty percent of patients $(n=132)$ showed reverse remodeling at discharge and percent of patients showing LVRR increased significantly at 
Table 2. Mitral regurgitation

\begin{tabular}{lllllll}
\hline & $\begin{array}{l}\text { Baseline } \\
\mathrm{n}=220\end{array}$ & $\begin{array}{l}\text { Discharge } \\
\mathrm{n}=220\end{array}$ & $\begin{array}{l}1 \text { Year } \\
\mathrm{n}=187\end{array}$ & $\begin{array}{l}3 \text { Years } \\
\mathrm{n}=122\end{array}$ & $\begin{array}{l}5 \text { Years } \\
\mathrm{n}=61\end{array}$ & ${ }^{*} \mathrm{p}$ \\
\hline $\mathrm{MR}$ & $3.1 \pm 0.6$ & $0.3 \pm 0.5^{*}$ & $0.3 \pm 0.6$ & $1.2 \pm 0.7^{\ddagger}$ & $2.1 \pm 1.2^{5}$ & 0.001
\end{tabular}

Direction of regurgitant jet

\begin{tabular}{|c|c|c|c|c|c|c|}
\hline Central & $70(31.8)$ & - & - & - & - & \\
\hline Anterior & $28(12.7)$ & - & - & - & - & \\
\hline Posterior & $54(24.6)$ & - & - & - & 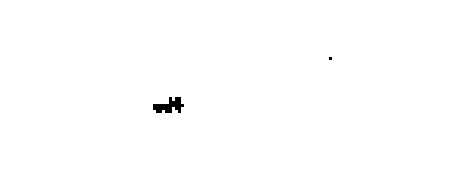 & \\
\hline Complex & $68(30.2)$ & - & - & - & - & \\
\hline \multicolumn{7}{|c|}{ Carpentier's classification } \\
\hline Type IIIb & $134(60.9)$ & - & - & - & - & \\
\hline $\begin{array}{l}\text { Type IIIb + annular } \\
\text { dilatation }\end{array}$ & $86(39.1)$ & - & - & - & - & \\
\hline \multicolumn{7}{|l|}{ Quantitative data } \\
\hline $\mathrm{TA}$ & $3.4 \pm 1.1$ & $1.9 \pm 0.5^{\circ}$ & $1.9 \pm 0.5$ & $2.1 \pm 0.6^{\ddagger}$ & $2.4 \pm 0.7^{5}$ & $<0.001$ \\
\hline ERO & $36 \pm 11$ & - & - & - & $19 \pm 11^{5}$ & - \\
\hline $\mathrm{RF}$ & $45 \pm 10$ & - & - & - & $26 \pm 14^{9}$ & - \\
\hline RV & $56 \pm 13$ & - & - & - & $29 \pm 16^{5}$ & - \\
\hline $\mathrm{CH}$ & $1.2 \pm 0.3$ & $0.8 \pm 0.2^{5}$ & $0.8 \pm 0.2$ & $0.9 \pm 0.3$ & $0.9 \pm 0.3$ & 0.02 \\
\hline $\mathrm{MA}_{s}$ & $4.1 \pm 1.2$ & $2.6 \pm 0.7^{*}$ & $2.6 \pm 0.4$ & $2.6 \pm 0.7$ & $2.6 \pm 0.7$ & 0.001 \\
\hline $\mathrm{MA}_{\mathrm{d}}$ & $5.0 \pm 1.1$ & $3.3 \pm 0.5^{*}$ & $3.4 \pm 0.6$ & $3.4 \pm 0.9$ & $3.4 \pm 0.9$ & $<0,001$ \\
\hline $\mathrm{M} \Delta \mathrm{p}$ & - & $2.3 \pm 0.5$ & $2.4 \pm 0.3$ & $2.3 \pm 0.3$ & $2.3 \pm 0.3$ & 0.2 \\
\hline
\end{tabular}

Continuous variables are presented as mean \pm standard deviation. Discrete data are presented as $\mathrm{n}(\%)$. $\mathrm{p}=\mathrm{ANOVA}$ over time significance. ${ }^{* \neq \ddagger \S}$ Significance vs. preoperative, discharge, 1 year, 3 years, respectively (see text). Abbreviations: $M R=$ Mitral Regurgitation; $T A=$ Tenting area $\left(\mathrm{cm}^{2}\right) ; E R O=$ effective regurgitant orifice $\left(\mathrm{mm}^{2}\right)$; RF=Regurgitant fraction (\%); RV=Regurgitant volume (ml); $\mathrm{CH}=$ Coaptation height $(\mathrm{cm})$; MAs=(Systolic) Mitral annular area $\left(\mathrm{cm}^{2}\right) ; \mathrm{MA}_{\mathrm{d}}=\left(\right.$ Diastolic) Mitral annular area $\left(\mathrm{cm}^{2}\right)$; $\mathrm{M} \Delta \mathrm{p}=$ Trans mitral diastolic gradient $(\mathrm{mmHg})$;

1 -year $(\mathrm{p}<0.001)$, but LVRR was reduced at 3-year $(\mathrm{p}<0.001)$ and at 5 -year $(\mathrm{p}=0.01)$ studies (Figure 3).

\section{LOCAL LV REMODELING}

For local remodeling (Table 4), indexes of posterior and lateral displacement of both papillary muscles, PMseparation, PPM fibrosis and $\mathrm{WMSI}_{s}$ were reduced significantly at discharge and remained constant at 1 year; nevertheless, PPM posterior ( 3 years, $\mathrm{p}=0.01 ; 5$ years, $\mathrm{p}=0.02$ ), PPM lateral ( 3 years, $\mathrm{p}=0.008 ; 5$ years, $\mathrm{p}=0.02$ ), $\mathrm{PM}_{\mathrm{s}}$ separation (3 years, $\mathrm{p}<0.001 ; 5$ years, $\left.\mathrm{p}=0.02\right)$ and PPM fibrosis $(\mathrm{p}<0.001)$ 


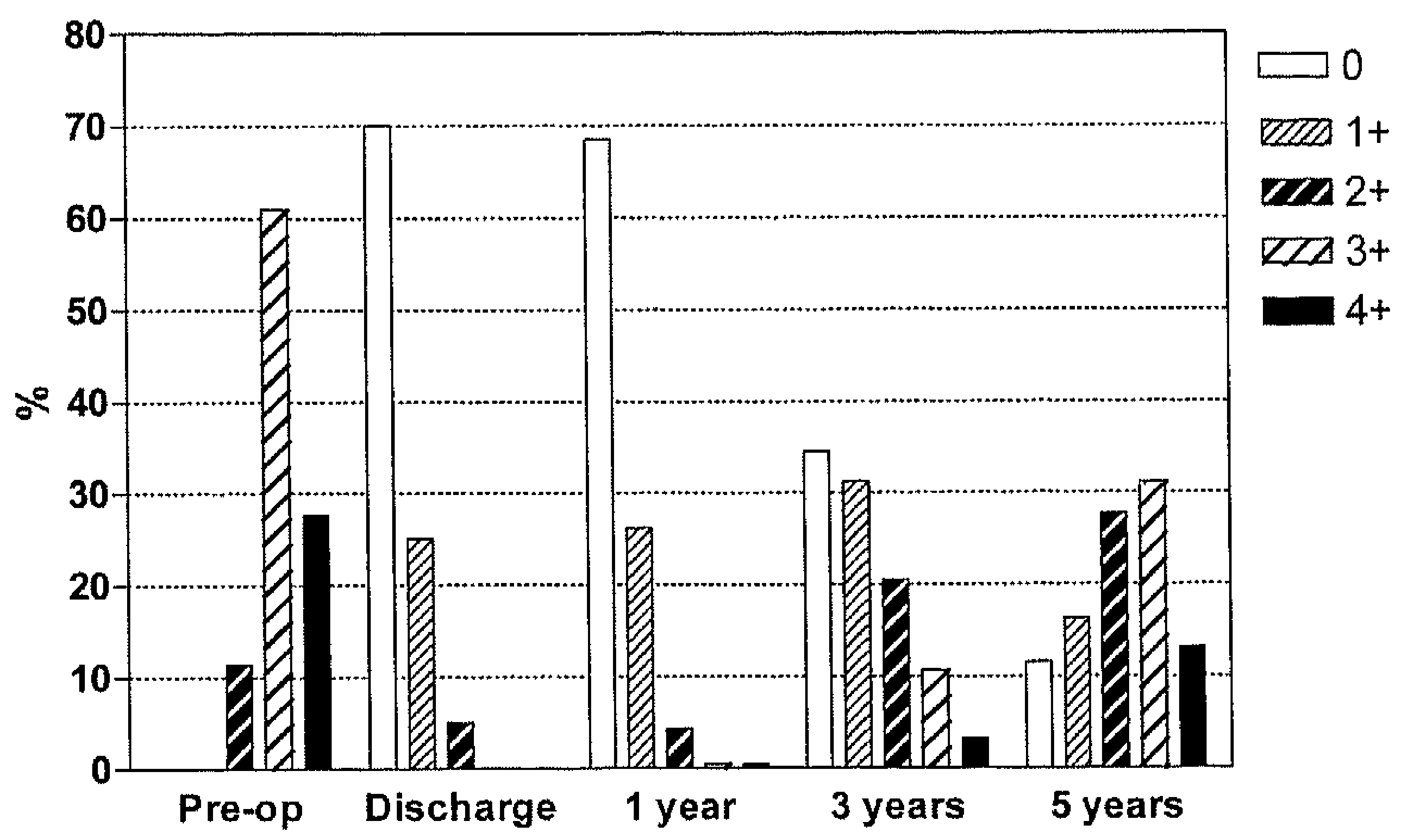

Figure 3. Mitral Regurgitation (\%) at different echocardiographic controls ( $<<.001)$..

\section{Freedom from Reoperation}

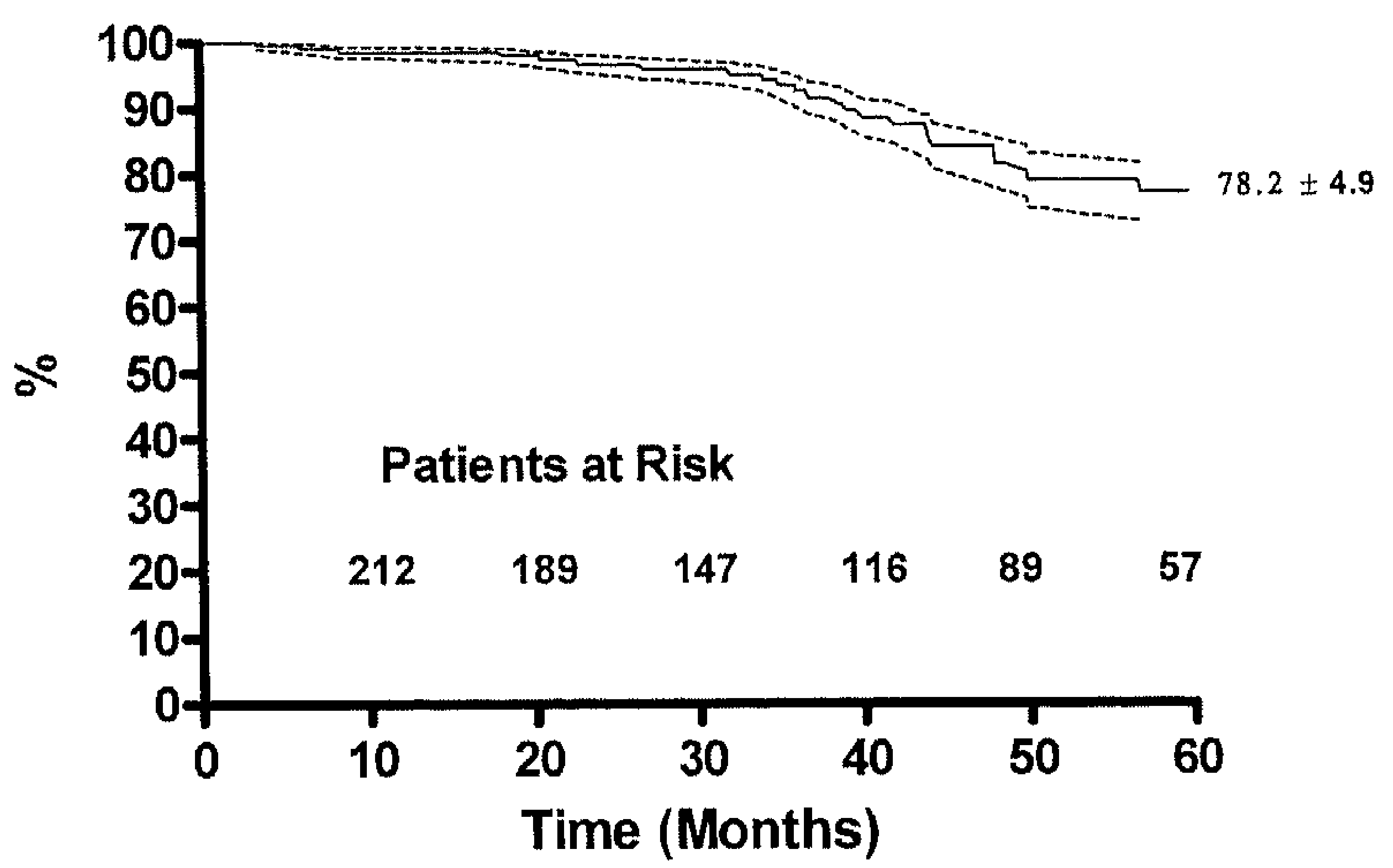

Figure 4. Five-year freedom from reoperation for recurrent ischemic mitral regurgitation (Dotted lines, 95\% Confidence interval).. 
Table 3. Global LV remodeling.

\begin{tabular}{lcccccc}
\hline & Baseline & Discharge & 1 Year & 3 Years & 5 Years & p \\
\hline EDD & $62 \pm 7$ & $54 \pm 7^{*}$ & $55 \pm 7$ & $53 \pm 7$ & $54 \pm 7$ & 0.03 \\
ESD & $52 \pm 8$ & $44 \pm 6^{\circ}$ & $43 \pm 6$ & $42 \pm 6$ & $43 \pm 6$ & 0.04 \\
LA & $50 \pm 7$ & $45 \pm 3^{*}$ & $46 \pm 3$ & $46 \pm 3$ & $46 \pm 4$ & 0.053 \\
ESV & $150 \pm 24$ & $114 \pm 15^{\circ}$ & $108 \pm 16^{\dagger}$ & $121 \pm 11^{\ddagger}$ & $136 \pm 11^{5}$ & $<0.001$ \\
EDV & $195 \pm 35$ & $164 \pm 26^{\circ}$ & $156 \pm 19^{\dagger}$ & $171 \pm 23^{\ddagger}$ & $191 \pm 23^{\varsigma}$ & 0.02 \\
LVEF & $30 \pm 12$ & $31 \pm 10$ & $30 \pm 10$ & $30 \pm 11$ & $29 \pm 12$ & 0.38 \\
MPI & $0.79 \pm 0.2$ & $0.76 \pm 0.1$ & $0.75 \pm 0.1$ & $0.75 \pm 0.1$ & $0.76 \pm 0.1$ & 0.2 \\
SIs & $0.65 \pm 0.2$ & $0.50 \pm 0.1^{*}$ & $0.50 \pm 0.1$ & $0.58 \pm 0.1^{\ddagger}$ & $0.66 \pm 0.2^{\ddagger}$ & 0.03 \\
SId & $0.72 \pm 0.1$ & $0.62 \pm 0.1^{*}$ & $0.60 \pm 0.1$ & $0.66 \pm 0.1^{\ddagger}$ & $0.74 \pm 0.1^{\$}$ & 0.01 \\
WMSI & $1.4 \pm 0.7$ & $1.2 \pm 0.3^{*}$ & $1.1 \pm 0.2$ & $1.3 \pm 0.4^{\ddagger}$ & $1.5 \pm 0.5^{\S}$ & 0.006 \\
\hline
\end{tabular}

Continuous variables are presented as mean \pm standard deviation. $\mathrm{p}=\mathrm{ANOVA}$ over time significance. ${ }^{* t \neq 5}$ Significance vs. preoperative, discharge, 1 year, 3 years, respectively (see text).

Abbreviations: $\mathrm{EDD}=($ Left ventricular) End diastolic diameter $(\mathrm{mm}) ; \mathrm{ESD}=($ Left ventricular) End systolic diameter $(\mathrm{mm}) ; \mathrm{LA}=\mathrm{Left}$ atrium $(\mathrm{mm})$; ESV=(Left ventricular) End systolic volume $(\mathrm{ml})$; $E D V=(L e f t$ ventricular) End diastolic volume $(\mathrm{ml}) ; L V E F=L e f t$ ventricular ejection fraction (\%); MPI=Myocardial performance Index; $S I_{S}=$ Systolic sphericity Index; $S I_{D}=$ Diastolic sphericity Index; WMSI=Wall motion score Index.

Table 4. Local LV remodeling

\begin{tabular}{lcccccc}
\hline & Baseline & Discharge & 1Year & 3 Years & 5 Years & P \\
\hline PPM posterior D & $2.6 \pm 0.6$ & $2.2 \pm 0.5^{*}$ & $2.1 \pm 0.3$ & $2.3 \pm 0.3^{\ddagger}$ & $2.5 \pm 0.6^{\$}$ & 0.02 \\
APM posterior D & $2.9 \pm 0.5$ & $2.5 \pm 0.2^{*}$ & $2.4 \pm 0.3$ & $2.4 \pm 0.3$ & $2.4 \pm 0.3$ & $<0.001$ \\
PPM lateral D & $2.0 \pm 0.4$ & $1.6 \pm 0.3^{*}$ & $1.6 \pm 0.3$ & $1.8 \pm 0.3^{\ddagger}$ & $2.1 \pm 0.3^{\S}$ & 0.01 \\
APM lateral D & $1.3 \pm 0.3$ & $1.0 \pm 0.2^{*}$ & $1.1 \pm 0.3$ & $1.1 \pm 0.3$ & $1.1 \pm 0.3$ & 0.008 \\
PM separation & $3.6 \pm 0.5$ & $3.2 \pm 0.2^{*}$ & $3.0 \pm 0.2$ & $3.3 \pm 0.2^{\ddagger}$ & $3.5 \pm 0.4^{\S}$ & $<0.001$ \\
PPM-fibrosa D & $6.7 \pm 0.6$ & $6.0 \pm 0.4^{*}$ & $6.0 \pm 0.4$ & $6.4 \pm 0.4^{\ddagger}$ & $6.6 \pm 0.5^{\S}$ & 0.02 \\
PPM WMSI & $2.2 \pm 0.2$ & $2.0 \pm 0.2^{*}$ & $2.1 \pm 0.2$ & $2.1 \pm 0.2$ & $2.1 \pm 0.2$ & 0.03 \\
APM WMSI & $1.3 \pm 0.4$ & $1.0 \pm 0.1^{*}$ & $1.0 \pm 0.1$ & $1.1 \pm 0.1$ & $1.1 \pm 0.1$ & 0.003 \\
\hline
\end{tabular}

Continuous variables are presented as mean \pm standard deviation. $p=A N O V A$ over time significance. $* \ddagger \$$ Significance vs. preoperative, discharge, 1 year, 3 years, respectively. (see text).

Abbreviations: $D=$ Distance $(\mathrm{cm})$; PPM=Posterior papillary muscle; $A P M=$ Anterior papillary muscle; PMs Papillary muscles; WMSI=Wall motion Score Index. 
LVRR

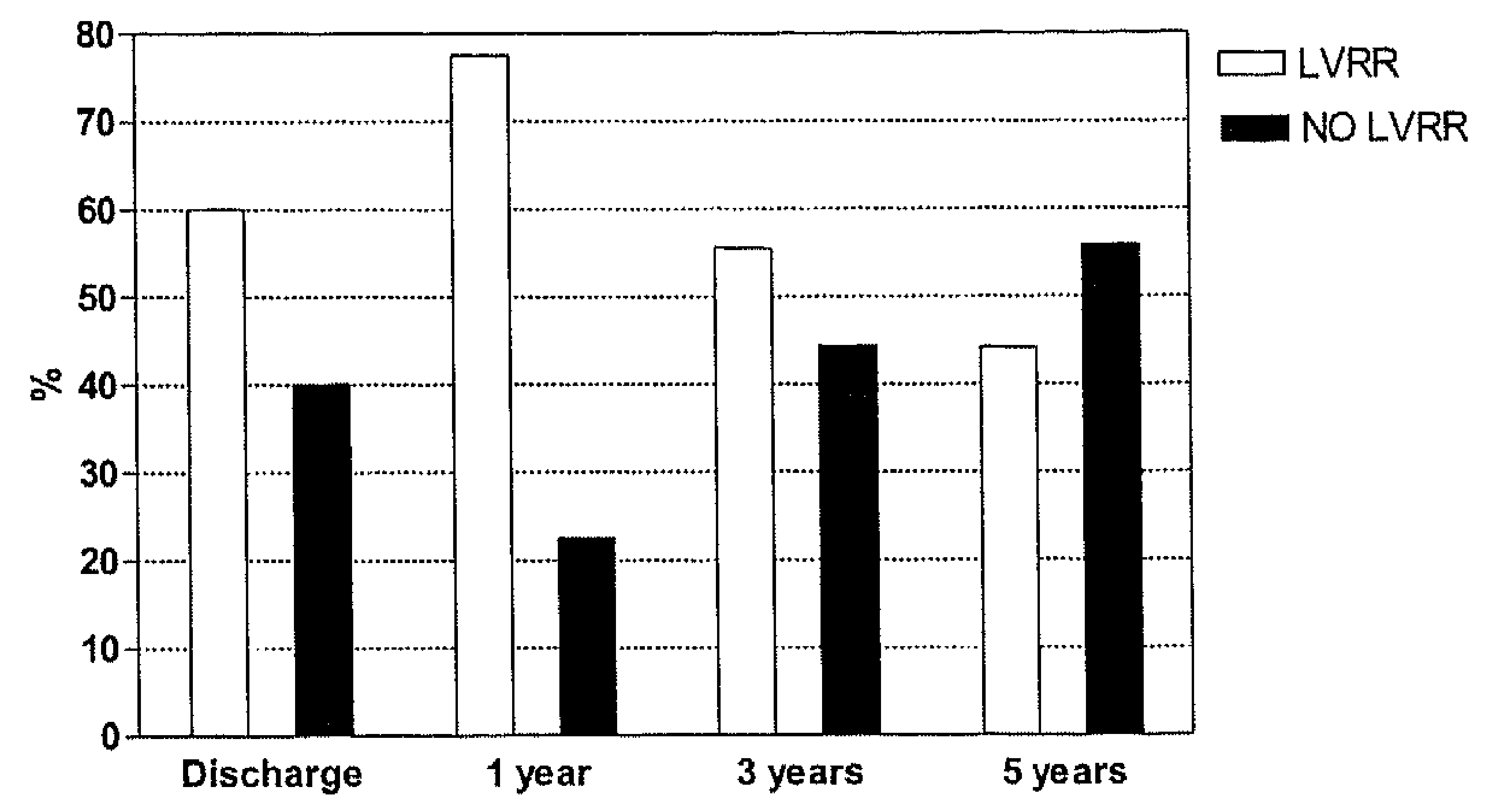

Figure 5. Left ventricular reverse remodeling $(\mathrm{p}=0.02)$.

re-increased significantly at subsequent controls. Contrastingly, lateral and posterior displacements of the anterior papillary muscle as well as wall motion score indexes did not show further significant changes.

\section{Predictors of Recurren't MR}

The unadjusted relationship between recurrent mitral regurgitation and independent variables are shown in Table 5. Multivariable logistic regression analysis identified systolic sphericity Index (OR=4.97, 95\% CI $=2.11-6.40, \mathrm{p}<0.001)$, myocardial performance Index $(\mathrm{OR}=4.66,95 \% \mathrm{Cl}=2.01-5.43, \mathrm{p}<0.001)$, end systolic volume $(\mathrm{OR}=2.59,95 \% \mathrm{CI}=1.19-3.77, \mathrm{p}=0.003)$ and wall motion score Index $(\mathrm{OR}=1.99$, $95 \% \mathrm{CI}=0.88-2.37, \mathrm{p}=0.04)$ as independent predictors of the occurrence of recurrent $\mathrm{MR}$

Internal validation of such multivariable analysis by means of bootstrapping, including the same variables of standard logistic regression model, further confirmed the significant predictive of recurrent MR (bootstrap $\mathrm{OR}=2.3,95 \%$ bias-corrected 95\% CI=1.1-5.2). Finally, logistic regression model was reliable (Hosmer-Lemeshow test, $\mathrm{p}=0.7$ ) and accurate (c-index=0.81).

ROC analysis showed that $\mathrm{SI}_{S}$ had $100 \%$ of sensitivity and specificity with an optimal cutoff $\geq 0.7$ ( $95 \%$ CI by bootstrapping $0.58-0.81$ ) [area under curve $1 ; 95 \%$ CI $0.70-1 ; \mathrm{p}<0.001)$. MPI with a cutoff $\geq 0.9$ ( $95 \% \mathrm{CI}$ by bootstrapping $0.75-0.96)$ had $85 \%$ of sensitivity and $84 \%$ of specificity (area under curve $0.94 ; 95 \%$ CI $0.67-1$; $\mathrm{p}<0.001)$. In addition, ESV $\geq 145 \mathrm{ml}(95 \% \mathrm{Cl}$ by bootstrapping $134-155)$ had $90 \%$ of sensitivity and specificity (area under curve 0.87; 95\% CI 0.80-0.94; $\mathrm{p}<0.001$ ). 
Table 5. Bivariate relationship of Independent variables with recurrent MR

\begin{tabular}{|c|c|c|c|}
\hline & $\mathrm{MR}+$ & MR- & $p$ \\
\hline Age (years) & $69.2 \pm 6.4$ & $67.8 \pm 8.3$ & 0.25 \\
\hline Male gender & $98(62.1)$ & $38(65.5)$ & 0.9 \\
\hline NYHA class & $3.2 \pm 0.5$ & $3.2 \pm 0.5$ & 0.74 \\
\hline CCS angina class & $3.0 \pm 0.3$ & $3.1 \pm 0.3$ & 0.32 \\
\hline \multicolumn{4}{|l|}{ Euroscore } \\
\hline Additive & $7.9 \pm 3.5$ & $8.1 \pm 4.6$ & 0.66 \\
\hline Logistic & $12.9 \pm 6.3$ & $12.8 \pm 5.5$ & 0.88 \\
\hline Hypertension & $63(39.9)$ & $25(40.3)$ & $>0.9$ \\
\hline Diabetes & $51(32.2)$ & $17(21.4)$ & 0.49 \\
\hline COPD & $21(13.2)$ & $7(11.2)$ & 0.71 \\
\hline Chronic renal disease & $20(12.6)$ & $4(6.5)$ & 0.18 \\
\hline Cerebral vascular disease & $10(6.4)$ & $4(6.5)$ & 0.9 \\
\hline Peripheral vascular disease & $8(5.1)$ & $2(3.3)$ & 0.6 \\
\hline Familiar history & $67(42.4)$ & $31(50.0)$ & 0.31 \\
\hline $\mathrm{MI}$ posterior/inferior & $79(50.0)$ & $28(45.1$ & 0.6 \\
\hline Grade of MR & $3.0 \pm 0.4$ & $3.1 \pm 0.4$ & 0.1 \\
\hline Central direction of regurgitant jet & $49(31.0)$ & $21(33.8)$ & 0.8 \\
\hline Annular dilatation & $66(41.7)$ & $20(32.2)$ & 0.19 \\
\hline TA & $3.3 \pm 1.1$ & $3.6 \pm 1.2$ & 0.06 \\
\hline ERO & $38.3 \pm 13$ & $36.1 \pm 11$ & 0.09 \\
\hline $\mathrm{RF}$ & $45.6 \pm 11$ & $44.7 \pm 12$ & 0.61 \\
\hline RV & $57.4 \pm 13$ & $55.0 \pm 10$ & 0.25 \\
\hline $\mathrm{CH}$ & $1.3 \pm 0.2$ & $1.1 \pm 0.1$ & 0.004 \\
\hline $\mathrm{MA}_{s}$ & $4.1 \pm 1.2$ & $4.3 \pm 1.2$ & 0.3 \\
\hline $\mathrm{MA}_{\mathrm{d}}^{*}$ & $4.9 \pm 1.2$ & $5.2 \pm 1.2$ & 0.11 \\
\hline Number of diseased coronary vessels & $2.5 \pm 0.4$ & $2.4 \pm 0.4$ & 0.11 \\
\hline Left main coronary artery & $28(17.7)$ & $8(12.9)$ & 0.3 \\
\hline Preoperative IABP & $6(3.7)$ & $2(3.2)$ & 0.88 \\
\hline Ring size & $27.6 \pm 0.5$ & $27.3 \pm 0.9$ & 0.21 \\
\hline Anastomoses/patient & $2.2 \pm 1.2$ & $2.1 \pm 1.0$ & 0.51 \\
\hline Arterial Graft/patient & $1.09 \pm 0.1$ & $1.06 \pm 0.1$ & 0.43 \\
\hline CCL time & $124.4 \pm 30$ & 123.28 & 0.8 \\
\hline $\mathrm{CPB}$ time & $92.7 \pm 16$ & $90.7 \pm 18$ & 0.61 \\
\hline EDD & $65.1 \pm 4$ & $60.6 \pm 5$ & 0.003 \\
\hline ESD $\quad \vdots$ & $53.9 \pm 4$ & $51.3 \pm 3$ & 0.01 \\
\hline LA dimensions & $49.2+7$ & $50.3 \pm 7$ & 0.66 \\
\hline ESV & $164.7 \pm 23.9$ & $131.2 \pm 19.8$ & $<0.001$ \\
\hline $\mathrm{EDV}$ & $215.3 \pm 38.1$ & $194.1 \pm 39.2$ & 0.09 \\
\hline LVEF & $24.4 \pm 7$ & $34.1 \pm 6$ & $<0.001$ \\
\hline MPI & $0.90 \pm 0.15$ & $0.69 \pm 0.17$ & $<0.001$ \\
\hline $\mathrm{SI}_{\mathrm{s}}$ & $074 \pm 0.1$ & $0.54 \pm 0.1$ & $<0.001$ \\
\hline $\mathrm{SI}_{\mathrm{D}}$ & $0.80 \pm 0.1$ & $0.64 \pm 0.09$ & 0.002 \\
\hline
\end{tabular}




\begin{tabular}{llll}
\hline & MR & MR- & p \\
\hline WMSI & $1.6 \pm 0.2$ & $1.1 \pm 0.1$ & $<0.001$ \\
PPM posterior D & $2.4 \pm 0.4$ & $2.6 \pm 0.5$ & 0.2 \\
APM posterior D & $2.7 \pm 04$ & $2.9 \pm 0.5$ & 0.08 \\
PPM lateral D & $1.9 \pm 0.4$ & $2.0 \pm 0.4$ & 0.59 \\
APM lateral D & $1.3 \pm 0.3$ & $1.1 \pm 0.2$ & 0.008 \\
PM $_{S}$ separation & $3.6 \pm 0.5$ & $3.6 \pm 0.4$ & 0.74 \\
PPM fibrosa D & $6.7 \pm 0.6$ & $6.6 \pm 0.5$ & 0.25 \\
PPM WMSI & $2.0 \pm 0.1$ & $2.2 \pm 0.2$ & 0.069 \\
APM WMSI & $1.3 \pm 0.4$ & $1.1 \pm 0.3$ & 0.04 \\
\hline
\end{tabular}

Abbreviations: $M R+(-)=$ Presence (absence) of recurrent mitral regurgitation; NYHA=New York Heart Association; $C C S=$ Canadian Cardiovascular Society; $C O P D=C h r o n i c$ obstructive pulmonary disease; $M I=$ Myocardial infarction; $M R=$ Mitral regurgitation; $M R=$ Mitral regurgitation; $\mathrm{CABG}=$ Coronary artery bypass grafting; TA=Tenting area $\left(\mathrm{cm}^{2}\right) ; \mathrm{ERO}=$ effective regurgitant orifice $\left(\mathrm{mm}^{2}\right) ; \mathrm{RF}=$ Regurgitant fraction (\%);RV=Regurgitant volume (ml/beat));CH=Coaptation heigh(mm); MAs $=$ Systolic mitral annular area $\left(\mathrm{cm}^{2}\right) ; \mathrm{MAd}=$ Diastolic mitral annular area $\left(\mathrm{cm}^{2}\right) ;$ IABP=Intaaortic balloon pump; $\mathrm{CPB}=$ Cardiopulmonary bypass; $\mathrm{CCL}=$ (Aortic).

Cross-clamp; $\mathrm{EDD}=\mathrm{End}$ diastolic diameter $(\mathrm{mm}) ; \mathrm{ESD}=\mathrm{End}$ systolic diameter $(\mathrm{mm}) ; \mathrm{LA}=\mathrm{Left}$ atrium $(\mathrm{mm}) ; \mathrm{ESV}=$ End systolic volume $(\mathrm{ml}) ; \mathrm{EDV}=$ End diastolic volume $(\mathrm{ml}) ; \mathrm{LVEF}=$ Left ventricular ejection fraction (\%); MPI=Myocardial performance Index; SIS=Systolic sphericity Index; SID=Diastolic sphericity Index; WMSI=Wall motion score Index; $\mathrm{D}=\mathrm{Distance}(\mathrm{mm})$; $\mathrm{PPM}=$ Posterior papillary muscle; $\mathrm{APM}=$ Anterior papillary muscle; $\mathrm{PMs}=$ Papillary muscles.

Finally, WMSI $\geq 1.5$ (95\% CI by bootstrapping $1.35-1.62$ ) had $80 \%$ of sensitivity and $82 \%$ of specificity (area under curve $0.81 ; 95 \%$ CI $0.77-0.91 ; \mathrm{p}=0.002$ ).

\section{DISCUSSION}

An established therapeutic approach to relieve CIMR, in association with coronary artery bypass grafting (CABG), is and undersized mitral ring annuloplasty (UMRA) ${ }^{2}$ which, by reducing the septal-lateral dimensions and the valve area, brings both mitral leaflets into apposition. Nonetheless, after encouraging initial results ${ }^{26}$, different studies have revealed a significant proportion of patients developing recurrent MR during follow-up ${ }^{4,7,27}$ which has been mainly related to continued LV remodeling and persistent leaflet tethering ${ }^{5,28}$. We reported our experience with this technique. Patients were followed-up by serial TTE over a 5-year period and recurrence of MR, local and global LV remodeling and LV reverse remodeling were explored. We also identified potential useful predictors of MR-recurrence following combined UMRA and CABG.

Because of unavailable complete echocardiographic data, 15 patients who died late after surgery were excluded from the study. 
In our report, recurrent MR significantly increased at 3 year and 5-year controls with and $27.8 \%$ of patients showing mild, $31.1 \%$ moderate and $13.1 \%$ severe MR at latest follow-up. Five-year freedom from reoperation for failed repair was $78.2 \pm$ $4.9 \%$. Hung and coworkers 5 showed $72 \%$ of patients with moderate/severe MR at late control ( $47 \pm 27$ months) after ring annuloplasty and CABG. Accordingly, Tahta et $\mathrm{al}^{27}$ in a large single-center surgical series, reported a $29 \%$ incidence of $M R \geq 2$ over a 3-year follow-up period. However, in these studies annuloplasty had not been routinely performed in a restrictive fashion. In contrast with these results, Braun and coworkers ${ }^{4}$ reported, in 87 patients undergoing restrictive ring annuloplasty (2 ring sizes, median ring size 26), no patient showing $M R \geq 3$ and 2 with $M R$ graded $2+$ at 18 -month follow-up. In addition, Geidel ${ }^{29}$ and co-workers reported 15 of 29 patients having no MR (52\%), nine patients with MR1+ (31\%) and five patients MR2+ (17\%) $13 \pm 7$ months after mitral annuloplasty and CABG. Of interest, they performed mitral downsizing in a "dynamic fashion" dependent on LV function (2, 3 or 4 ring sizes for $L V E F>30,20-30 \%,<20 \%$, respectively).

\section{REMODELING}

In our experience, we observed a significant global remodeling late after reductive annuloplasty: LV volumes increased significantly and WMSI exceeded its preoperative value. Furthermore, LV became more spherical with sphericity indexes exceeding their baseline values at 5 -year echocardiography. Regarding local remodeling, we observed a late "asymmetric" lateral and posterior displacement of PPM with APM displacement which remained constant over time. Of interest, WMSI of the segments underlying both papillary muscles, after a transient reduction, were relatively stable and these data suggest that the late PPM displacement seems to be due to the continued global remodeling, rather than local remodeling in this area.

\section{LVRR}

According to Stellbrink and coworkers ${ }^{19}$, we considered a decrease in LV end systolic volume $>15 \%$ from baseline value as LVRR. In this fashion LVRR was observed, at late control, in $44.2 \%$ of patients with $10.3 \%$ showing further increment in ESV. Braun and coworkers ${ }^{6}$ reported $60.5 \%$ of patients with significant reverse LV remodeling 18 months after restrictive mitral annuloplasty. We had comparable results at 12 -month follow-up (77.5\%) but LVRR dramatically worsened at late controls. However, our data are not comparable because this Group considered a 10\% reduction in EDD as significant LVRR; However, measurements of LV 
volumes have been demonstrated to be more reliable than LV diameters in assessing LV remodeling, especially in enlarged ventricles ${ }^{30}$.

\section{Predictors of ReCURRent MR}

At multivariable model, ESV $\geq 145 \mathrm{ml}, \mathrm{SI}_{s} \geq 0.7, \mathrm{MPI} \geq 0.9$ and WMSI $\geq 1.5$ were predictors of recurrent MR. These results emphasize the importance of the extent of preoperative LV global remodeling as having a central role in predicting inadequate results after UMRA. A new finding in this study is that MPI predicts the likelihood of MR, whereas LVEF was not significant. The MPI is a combined systolic and diastolic Doppler-derived index for the assessment of global LV performance which, differently from LVEF, is independent of LV loading conditions and heart rate.

\section{STUdY LIMITATION}

Our study findings should be viewed in light of some inherent limitations.

- Evaluation of LVRR was based on volumes obtained by echocardiography; Volumetry by $2 \mathrm{D}$ echocardiography depends on geometric assumptions and is subject to image-plane positioning errors. Hence it is not accurate in left ventricles that are distorted in shape such as after myocardial infarction. However, this limitation belongs to most of published paper regarding this pathology.

- Viability testing was not performed in these patients. Therefore lack of LVRR in non responders might be also due to irreversible ischemic myocardial damage (not-viable myocardium). This issue deserves further investigation.

- Postoperative evaluation of the coronary status was not assessed. It would have been helpful to differentiate between surgical failure (valve repair and $C A B G$ ) and the progress of the coronary disease.

- Fifteen patients with late deaths were not included in the study. They could have benefited from serial echocardiograms possibly with different results.

- Estimated cutoffs are known to be very susceptible to changes in the study population. We employed bootstrapping techniques to validate the results, nonetheless, it has also been documented that the sensitivity/specificity associated with these cutoffs are overly optimistic.

\section{STRENGTH OF THE STUDY}

As far as we know, this is the largest cohort reported with UMRA in CIMR patients with detailed echocardiographic follow-up. Furthermore our patient cohort was more homogeneous than in other study ${ }^{31}$ : all patients underwent associated $C A B G$, they had no concomitant $M V$ procedures, and the entire cohort was uniform regarding the MV ischemic leaflet dysfunction. Moreover, to assess results 
for MV repair, we studied true "recurrent" MR excluding those patients with "residual" MR in whom the insufficiency was presumably never eliminated at surgery. Additionally, we undertook valve sizing in a standardized fashion and the degree of undersizing was homogeneous over the 5-year period of the study. Finally, we used only two rings (Carpentier's rigid or semi-flexible rings).

\section{Clinical Implications}

In our experience UMRA does not ensure successful and durable elimination of MR in all patients. Those who can benefit from combined UMRA and CABG should be preoperatively identified utilizing echo predictors of recurrent MR. However, restrictive annuloplasty resulted to be ineffective in a large percentage of patients and results of this study suggest the need for different approaches directly addressing ventricular tethering in most of CIMR patients ${ }^{32-35}$. Such procedures may include LV plasty with volume reduction, chordal elongation or cutting, PM displacement and leaflet elongation techniques as well as cardiac the employment of support devices introduced in recent years in clinical practice ${ }^{36,37}$. Mitral valve replacement, largely employed in the past in ischemic regurgitation, although eliminates the short-term risk of recurrent $M R$, it is associated with poor long-term survival ${ }^{38}$. Thus, in our actual policy, we do not consider MV replacement as a reasonable alternative to repair.

\section{CONCLUSIONS}

Combined UMRA and CABG provided poor mid-term results with significant recurrent $M R$. Our findings emphasize the need for improved repair technique and better patient selection to identify patients with anticipated repair failure who could benefit more from valve replacement or other procedure directly addressing ventricular tethering. Long term prospective studies on this controversial issue are encouraged.

\section{REFERENCES}

1. Miller DC. Ischemic mitral regurgitation redux-to repair or to replace? J Thorac Cardiovasc Surg 2001;122 (6):1059-62.

2. Bolling SF, Pagani FD, Deeb GM, Bach DS. Intermediate term outcome of mitral reconstruction in cardiomyopathy. J Thorac Cardiovasc Surg 1998; 115(2):381- 6. 
3. Bax JJ, Braun J, Somer ST, Klautz R, Holman ER, Versteegh MI, Boersma E, Schalij MJ, van der Wall EE, Dion RA. Restrictive annuloplasty and coronary revascularization in ischemic mitral regurgitation results in reverse left ventricular remodeling.Circulation.2004; 110[suppl II]:II103-8.

4. McGee EC, Gillinov AM, Blackstone EH, Rajeswaran J, Cohen G, Najam F, Shiota T, Sabik JF, Lytle BW, McCarthy PM, Cosgrove DM. Recurrent mitral regurgitation after annuloplasty for functional ischemic mitral regurgitation. J Thorac Cardiovasc Surg 2004, 128(6):916-24.

5. Hung J, Papakostas L, Tahta SA, Hardy BG, Bollen BA, Duran CM, Levine RA. Mechanism of recurrent ischemic mitral regurgitation after annuloplasty: continued LV remodeling as a moving target. Circulation 2004; 110[suppl.II]:I185- 90.

6. Braun J, Bax JJ, Versteegh MI, Voigt PG, Holman ER, Klautz RJ, Boersma E, Dion RA. Preoperative left ventricular dimensions predict reverse remodeling following restrictive mitral annuloplasty in ischemic mitral regurgitation. Eur J Cardiothorac Surg 2005; 27(5):847-53.

7. Serri K, Bouchard D, Demers P, Coutu M, Pellerin M, Carrier M, Perrault LP, Cartier R, Page $\mathrm{P}$, Cossette $\mathrm{M}$, Basmadjian AJ. Is a good perioperative echocardiographic result predictive of durability in ischemic mitral valve repair? J Thorac Cardiovasc Surg. 2006; 131(3):523-9.

8. Carpentier A. Cardiac valve surgery-The "French correction". J Thorac Cardiovasc. Surg. 1983; 86(3): 326-37.

9. World Medical Association Declaration of Helsinki: Ethical principles for medical research involving human subjects. Edinburgh, Scotland: World Medical Association, October 2000. Available ahttp//www.wma.net/e/policy/17-c_3html. Accessed June 22, 2001.

10. Jones $E$, Weintraub WS. The importance of completeness of revascularization during long-term follow-up after coronary artery operations. J Thorac Cardiovasc Surg 1996; 112:222-37.

11. Bland MJ, Altman DJ. Statistical method for assessing agreement between two methods of clinical measurements.Lancet 1986; 1:307-310.

12. Gardin JM, Adams DB, Douglas PS, Feigenbaum H, Forst DH, Fraser AG, Grayburn PA, Katz AS, Keller AM, Kerber RE, Khandheria BK, Klein AL, Lang RM, Pierard LA, Quinones MA, Schnittger I; American Society of Echocardiography. Recommendations for a standardized report for adult transthoracic echocardiography: a report from the American Society of Echocardiography's Nomenclature and Standards Committee and Task Force for a Standardized Echocardiography Report. J Am Soc Echocardiogr 2002; 15 (3):275-90.

13. Zoghbi WA, Enriquez-Sarano M, Foster E, Grayburn PA, Kraft CD, Levine RA, Nihoyannopoulos P, Otto CM, Quinones MA, Rakowski H, Stewart WJ, Waggoner A, Weissman NJ; American Society of Echocardiography. Recommendations for evaluation of the severity of native valvular regurgitation with two-dimensional and Doppler echocardiography. J Am Soc Echocardiogr 2003; 16 (7):777-802.

14. Enriquez-Sarano $M$, Tribouilloy $C$. Quantitation of mitral regurgitation: rationale approach and interpretation in clinical practice. Heart 2002, 88 (suppl IV):1-4.

15. Yiu SF, Enriquez-Sarano M, Tribouilloy C, Seward JB, Tajik AJ. Determinants of the degree of functional mitral regurgitation in patients with systolic left ventricular dysfunction: a quantitative clinical study. Circulation 2000, 102(12):1400-6. 
16. Boltwood CM, Tei C, Wong M, Shah PM. Quantitative echocardiography of the mitral complex in dilated cardiomyopathy: the mechanism of functional mitral regurgitation. Circulation 1983; 68(3):498-508.

17. Vijayaraghavan G, Boltwood CM, Tei C, Wong M, Shah PM. Simplified echocardiographic measurement of the mitral an nulus. Am Heart J 1986; 112(5): 985-91.

18. Schiller NB, Shah PM, Crawford $M$ et al. Recommendations for quantitation of the left ventricle by two-dimensional echocardiography. American Society of Echocardiography Committee on Standards, Subcommittee on Quantitation of Two-Dimensional Echocardiograms. J Am Soc Echocardiogr. 1989;2(5):358

19. Stellbrink C, Breithardt OA, Franke A, Sack S, Bakker P, Auricchio A, Pochet T, Salo R, Kramer A, Spinelli J; PATH-CHF (Pacing Therapies in Congestive Heart Failure) Investigators; CPI Guidant Congestive Heart Failure Research Group. Impact of cardiac resynchronization therapy using hemodynamically optimized pacing on left ventricular remodeling in patients with congestive heart failure and ventricular conduction disturbances. J Am Coll Cardiol. 2001; 38(7):195765.

20. Tei C, Ling LH, Hodge DO, Bailey KR, Oh JK, Rodeheffer RJ, Tajik AJ, Seward JB. New index of combined systolic and diastolic myocardial performance: a simple and reproducible measure of cardiac function a study in normals and dilated cardiomyopathy. J Cardiol 1995; 26(6):357-66.

21. Kono T, Sabbah HN, Rosman H, Alam M, Jafri S, Goldstein S. Left ventricular shape is the primary determinant of functional mitral regurgitation in heart failure. J Am Coll Cardiol. 1992; 20(7):1594-8.

22. Cerqueira MD, Weissman NJ, Dilsizian V, Jacobs AK, Kaul S, Laskey WK, Pennell DJ, Rumberger JA, Ryan T, Verani MS; American Heart Association Writing Group on Myocardial Segmentation and Registration for Cardiac Imaging. Standardized myocardial segmentation and nomenclature for tomographic imaging of the heart: a statement for healthcare professionals from the Cardiac Imaging Committee of the Council on Clinical Cardiology of the American Heart Association. Circulation. 2002; 105(4):539-42.

23. Grigioni F, Enriquez-Sarano M, Zehr KJ, Bailey KL, Tajik AJ. Ischemic Mitral Regurgitation. Long-term outcome and prognostic implications with quantitative Doppler assessment. Circulation 2001; 103(13):1759-64.

24. Harrel FE Jr, Lee KL, Mark DB. Multivariable Prognostic Models: Issues in developing models, evaluating assumptions and adequacy, and measuring and reducing errors. Stats Med 1996:36187.

25. Hosmer DW, Lemeshow S. Applied Logistic regression.2 $2^{\text {nd }}$ ed.New York: Wiley-Interscience; 2000:143-202.

26. Bolling SF, Deeb GM, Bach DS. Mitral valve reconstruction in elderly, ischemic patients. Chest 1996;109(1):35- 40.

27. Tahta SA, Oury JH, Maxwell JM, Hiro SP, Duran CM. Outcome after mitral valve repair for functional ischemic mitral regurgitation. J Heart Valve Dis. 2002;11(1):11-8; 
Five-Year Echocardiographic Results

28. Kuwahara E, Otsuji Y, Iguro Y, Ueno T, Zhu F, Mizukami N, Kubora K, Nakashiki K, Yuasa T, Yu B, Uemura T, Takasaki K, Miyata M, Hamasaki S, Kisanuki A, Levine RA, Sakata R, Tei C. Mechanism of recurrent/persistent ischemic/functional mitral regurgitation in the chronic phase after surgical annuloplasty: importance of augmented posterior leaflet tethering. Circulation. 2006 Jul 4; 114 (Suppl):I529-34.

29. Geidel S, Lass M, Schneider C, Groth G, Boczor S, Kuck KH, Ostermeyer J.. Downsizing of mitral valve and coronary revascularization in severe ischemic mitral regurgitation results in reverse left ventricular and left atrial remodeling. Eur J Cardiothorac Surg 2005;27:1011-16

30. Dujardin KS, Enriquez-Sarano M, Rossi A, Bailey KR, Seward JB. Echocardiographic assessment of left ventricular remodeling: are left ventricular diameters suitable tools? J Am Coll Cardiol. 1997 Nov 15; 30(6):1534-41.

31. Adams DH, Anyanwu A. Pitfalls and limitations in measuring and interpreting the outcomes of mitral valve repair. J Thorac Cardiovasc Surg. 2006; 131(3):523-9.

32. Barista RJ, Verde J, Nery P, Bocchino L, Takeshira N, Bhayana JN, Bergsland J, Graham S, Houck JP, Salerno TA.Partial left ventriculectomy to treat end-stage heart disease. Ann Thorac Surg. 1997; 64(3):634-8.

33. Dor V, Sabatier M, Di Donato M, Montiglio F, Toso A, Maioli M. Efficacy of endoventricular patch plasty in large postinfarction akinetic scar and severe left ventricular dysfunction: comparison with a series of large dyskinetic scars. J Thorac Cardiovasc Surg. 1998;116(1):50-9

34. Messas E, Guerrero JL, Handschumacher MD, Conrad C, Chow CM, Sullivan S, Yoganathan AP, Levine RA. Chordal cutting: a new therapeutic approach for ischemic mitral regurgitation. Circulation. 2001; 104(16):1958-63.

35. Tibayan FA, Rodriguez F, Langer F, Zasio MK, Bailey L, Liang D, Daughters GT, Ingels NB Jr, Miller DC.Does septal-lateral annular cinching work for chronic ischemic mitral regurgitation?] Thorac Cardiovasc Surg. 2004;127(3):654-63

36. Konertz W, Rombeck B, Hotz H. Short-term safety of the Acorn cardiac support device in patients with advanced heart failure. J Am Coll Cardiol 2000; 35:182A.

37. Fukamachi $\mathrm{K}, \mathrm{McC}$ arthy PM. Initial safety and feasibility clinical trial of the myosplint device. J Card Surg. 2005 Nov-Dec;20(6):S43-7

38. Gillinov AM, Wierup PN, Blackstone EH, Bishay ES, Cosgrove DM, White J, Lytle BW, McCarthy PM. Is repair preferable to replacement for ischemic mitral regurgitation? J Thorac Cardiovasc Surg. 2001;122(6):1125-41 


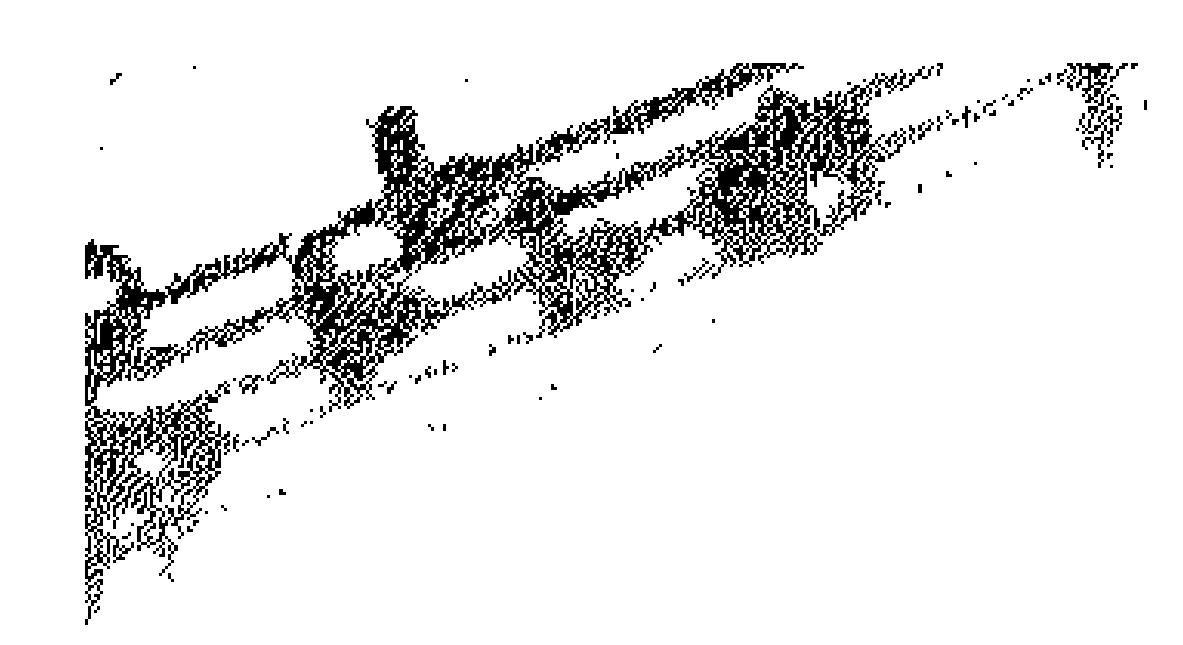

\section{Left Ventricular Reverse Remodeling After Undersized Mitral Ring Annuloplasty In Patients With Ischemic Regurgitation}

Sandro Gelsomino, MD, Roberto Lorusso, Ph.D, Irene Capecchi, MD, Carlo Rostagno, MD, Stefano Romagnoli, MD, Giuseppe Bille, MD, Giuseppe De Cicco, MD, Cecilia Tetta, MD,

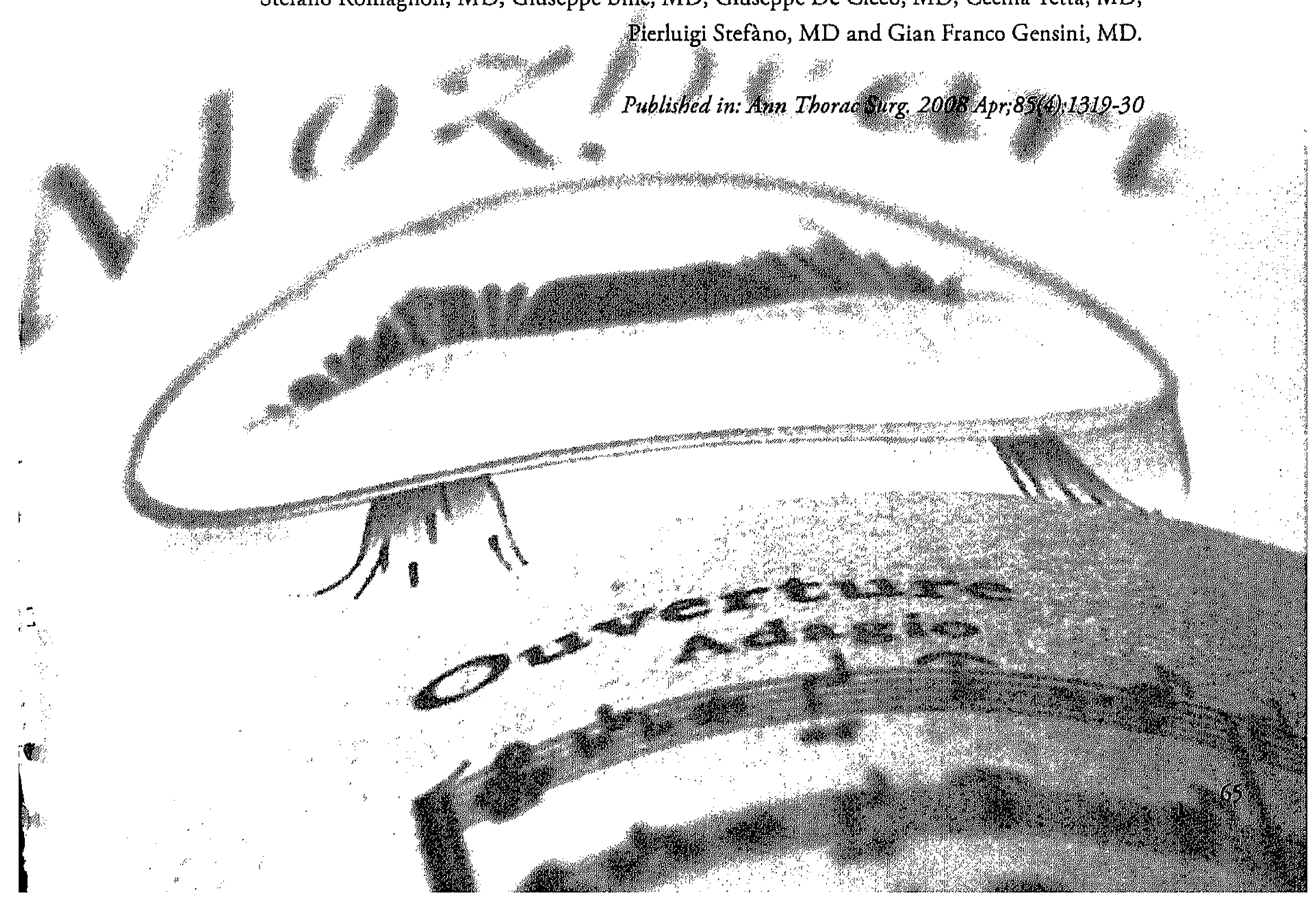




\section{ABSTRACT}

Background. Long-term durability of combined coronary arrery bypass grafting and of undersized mitral ring annuloplasty (UMRA) is uncertain with a considerable number of patients showing recurrent regurgitation. This study examines the difference in the benefit of UMRA on clinical end points and recurrence of mitral regurgitation between responders and non-responders of left ventricular reverse remodeling. Methods. Study eligibility criteria were fulfilled by 204 patients with chronic ischemic mirral regurgitation (CIMR) who survived combined coronary artery bypass and reductive annuloplasty between September 2001 and September 2006. Patients underwent echocardiography preoperatively, at discharge and at follow-up appointments (100\% complete; early, median 6 months [interquartile range 3-8 months]; late, 35 months [21-50 months]). Reverse remodeling was defined as a reduction in left ventricular end systolic volume Index $>15 \%$.

Results There were 84 (41.2\%) responders of reverse remodeling (age $68 \pm 7.4 ; 51$ males) and 120 (58.8\%) non-responders (age $67 \pm 7.6 ; 78$ males). Non-responders had a higher recurrence of mitral regurgitation $(p<0.001)$, higher reoperation rate for failed repair $(p<0.001)$ significantly larger left ventricular volumes and dimension at any study point $(\mathrm{p}<0.001)$ with significant late increase of sphericity indexes exceeding preoperative values $(p<0.001)$. At multivariable analysis baseline myocardial performance Index $<0.90(p<0.001)$, systolic sphericity Index $<0.72(p<0.001)$ and wall motion score Index $<1.59(\mathrm{p}=0.003)$ resulted as independent predictors of reverse remodeling.

Conclusions. Our experience suggests that more information on possible echo predictors of an inadequate result may improve preoperative decision-making of CIMR patients for UMRA. 


\section{INTRODUCTION}

Despite better understanding of mechanisms leading to chronic ischemic mitral valve regurgitation (CIMR) and notwithstanding improvement of mitral repair results ${ }^{1,2}$, ongoing dissatisfaction with outcomes has yielded different techniques ${ }^{3-6}$.

An established therapeutic approach to relieve CIMR, in association with coronary artery bypass grafting $(\mathrm{CABG})$, is undersized mitral ring annuloplasty (UMRA) ${ }^{7,8}$ which, by reducing the anterior-posterior dimensions (or septal-lateral in anatomic terms) and the valve area, brings both mitral leaflets into apposition. However, the long-term efficacy of UMRA is still unclear and recurrence of mitral regurgitation (MR) has been predominantly related to progressive left ventricular remodeling ${ }^{9-11}$. Cardiac remodeling is a well known important aspect of myocardial disease progression making left ventricular reverse remodeling (LVRR) a critical postoperative factor ${ }^{12}$.

This study examines the difference in the benefit of UMRA on clinical end points and recurrence of MR between responders and non-responders of LVRR. It also explores potential useful predictors of successful reverse remodeling including clinical and surgical data, volumetric and geometric parameters and indexes of left ventricular function assessed by echocardiography.

\section{MATERIAL AND METHODS}

\section{ETHICAL Issue}

Following the World Medical Association guidelines concerning ethical principles for medical research involving human subjects ${ }^{13}$, the study was approved by Institutional Ethics Board. Furthermore, all patients gave their informed consent.

\section{Patients population}

Chronic ischemic mitral regurgitation was defined as the combination of mildto-severe MR with 1) Prior myocardial infarction (MI) $>16$ days ${ }^{14}$; 2) seventy-five percent or greater stenosis of at least one coronary vessel; 3 ) a corresponding regional wall motion abnormality; 4) type IIIb leaflet dysfunction following Carpentier's classification ${ }^{15}$ with or without annular dilatation. Exclusion criteria were: 1) degenerative or other non-ischemic etiology; 2) ischemic isolated type I or type II dysfunction $^{15}$; 3) additional mitral valve repair procedures; 4) other valvular or congenital heart diseases; 5) previous cardiac surgery/percutaneous transluminal coronary angioplasty; 6) atrial fibrillation or sinus rhythm with heart rate at rest $>100$ bts/ $\min$. 
Among 239 Subjects with chronic ischemic mitral regurgitation undergoing combined CABG and UMRA in our Institution (Cardiac Surgery, Careggi Hospital, Florence, Italy) between September 2001 and September 2006 and prospectively enrolled in the study, 35 were excluded: 4 had intraoperative annuloplasty failure, 12 showed residual MR, defined as the insufficiency $\geq 2+$ following valve repair documented at discharge, 4 died within 30 days and 15 had incomplete data available.

\section{Patients Classification}

Following Stellbrink and coworkers ${ }^{16}$ patients were classified as responders in terms of LVRR if the end systolic volume Index (ESVI) was reduced $>15 \%$ at late follow-up compared with baseline volumes, and as non-responders if ESVI was reduced $\leq 15 \%$.

Among the 204 patients there were 84 (41.2\%) responders to LVRR and 120 $(58.8 \%)$ non-responders.

\section{Follow UP}

Clinical follow up information was obtained from all survivors through outpatient visits and phone calls and was $100 \%$ complete. Median follow up were: early, median 6 months, interquartile range [IQR] 3-8 months; late, 35 months, IQR 21-50 months).

\section{SURGERY}

All patients underwent associated CABG. For the purpose of this study, complete revascularization was accomplished when, at least one graft was placed distal to an approximately $50 \%$ diameter narrowing in each of the three major vascular system in which arterial narrowing of this severity was noted in a vessel $\geq 1.5 \mathrm{~mm}$ of diameter. It was not considered necessary to bypass all obstructed diagonal branches of the anterior descending or marginal branches of the circumflex coronary arteries for a classification of complete revascularization. Following this definition 100\% patients underwent complete revascularization.

The ring size was determined by standard measurements of the inter-trigonal distance and anterior leaflet height. A downsizing by two ting sizes was performed in all patients. Two types of ring were employed in all patients: Carpentier's classic ring (Edwards Life Sciences, Irvine, CA) in 112 patients (54.9\%) and Physio ring (Edwards Life Sciences, Irvine, CA) in 92 (45.1\%). Median ring size was $28 \mathrm{~mm}$ (Interquartile range [IQR] $26-28 \mathrm{~mm}$. No difference was detected in ring size between two types of ring employed (both, $28 \mathrm{~mm}$ (IQR 26-28 mm, p>0.9) 
After cardiopulmonary bypass (CPB), a transesophageal echocardiography (TEE) was performed to assess residual MR: leaflet coaptation $\geq 0.5 \mathrm{~cm}, M R \leq 1$ and systolic MV area $>2 \mathrm{~cm}^{2}$ was assessed as successful repair.

\section{Echocardiographic Measurements and Calculations}

The clinical echocardiographic evaluation was as follows: transthoracic (TTE) and transesophageal (TEE) echocardiograms were performed within five days before surgery. Then a transthoracic echocardiography was performed at discharge and at follow-up appointments. Echocardiographic studies were carried out on an Acuson Sequoia imaging device equipped with a $3.5-\mathrm{MHz}$ ultrasound transducer (Acuson Corporation, Mountain View, CA) preoperatively, at discharge and at follow-up appointments. The reliability of echocardiographic measurements was assessed by calculating between-observer (IC and CR) interval of agreements of main direct measures used in this study in a different group of 20 subjects $(10 \mathrm{MR})^{17}$.

\section{GLobal LV Remodeling}

LV volumes and left ventricular ejection fraction (LVEF) were assessed by the bi-apical Simpson disk method ${ }^{18}$. The myocardial performance index (MPI) was measured using the method described by Tei et al. ${ }^{19}$. The sphericity index (SI) was obtained at end diastole and end systole $\left(\mathrm{SI}_{\mathrm{D}}\right.$ and $\mathrm{SI}_{S}$, respectively) as the volume of the left ventricle divided by the volume of a sphere with a diameter equal to the longest axis of the left ventricle measured in the apical view ${ }^{20}$. The wall motion score index (WMSI) was calculated according to a 17-segment model ${ }^{21}$.

\section{Quantification of MR and Definitions}

The following quantitative measurements were simultaneously employed to grade the severity of MR and final results were averages of measured values ${ }^{1,22,23}$ : (1) Quantitative Doppler. (2) Proximal Isovelocity Surface Area (PISA). For each measurement a minimum of three cardiac cycles was averaged. The tenting area (TA) was measured by the area enclosed between the annular plane and mitral leaflets from the parasternal long-axis view at mid-systole. Apical displacement of the coaptation was measured as coaptation height $(\mathrm{CH})$ and coaptation length $(\mathrm{CL})$ was directly measured ${ }^{2,24}$. Mitral annular areas were obtained from mitral annular dimensions in apical long-axis, 4-chamber, and 2-chamber views, using an ellipsoid assumption ${ }^{25}$.

Recurrent MR was an insufficiency $\geq 2+$ at follow up appointments in patients with no/trivial $M R$ at discharge. 


\section{LOCAL LV REMODELING}

Lateral and posterior displacements of anterior papillary muscles (APM) and posterior papillary muscles (PPM) were measured as distances from well-defined anatomic landmarks at early and end systole ${ }^{2}$. Separation between papillary muscles was directly measured from body to body of $\mathrm{PM}_{s}$. In the long-axis view the apical displacement of the PPM was measured as the distance between the papillary muscle head and the fixed inter-valvular fibrosis (annular papillary distance). The WMSI of the basal and mid-posterior and inferior segments for the PPM and of basal and mid-lateral and anterior segments for the APM were calculated'.

\section{Statistical Analysis}

The sample size was determined by GraphPad StatMate release 2.00 (GraphPad Prism Software, Inc, San Diego, CA), on the basis of preliminary data obtained by echocardiography and was determined on the basis of the following assumptions: Type I error of 0.05 (two-sided), power of $80 \%$, difference in end systolic volume of $0.78 \pm 2.4$ between patients with or without recurrent mitral regurgitation.

The calculated study population was 200 . However 239 patients were recruited to allow for possible analytic problems while processing the eventualities potentially leading to patient attrition. Variables were tested for normal distribution by the Kolmogorov-Smirnov test. Continuous variables are presented as mean and standard deviation (SD), categorical variables as percentage of not-normally distributed variables as median and Irate bivariate association between the independent variables and LV reverse remodeling was assessed by the Student's t-test, Mann Whitney $U$ test, $\chi 2$ test and Fisher exact test were appropriate.

Echocardiographic variables over time were analyzed by means of ANOVA repeated measures followed by Tukey post-hoc test and Friedman test, where appropriate. Forty-nine demographic, clinical, operative and echocardiographic parameters were investigated for their predictive value of LVRR by univariable and multivariable models. To enhance the accuracy of the model, clustering method ${ }^{26}$ was employed to reduce the number of variables ${ }^{27}$.

Multivariable logistic regression analysis by means of a backward stepwise algorithm (cutoff for entry 0.05, for removal 0.10) was performed to select independent predictors of reverse remodeling. Categorical variables with more than two levels in the regression model were converted to dummy variables.

Model assumptions (linearity and additivity assumptions) were checked by piecewise cubic polynomials (spiline functions) and pooled interaction tes ${ }^{28}$, respectively, and found to be satisfied. "Goodness of fit" of the final logistic regression models was assessed with the Hosmer-Lemeshow statistic ${ }^{27,28}$ and predictive accuracy was assessed by the concordance (c) index ${ }^{27,28}$. Internal validation of predictors generated 
by multivariable logistic regression was performed by means of bootstrapping techniques, with 1000 cycles and generation of OR and bias corrected $95 \% \mathrm{CI}^{28,29}$.

Optimal cutoff values were determined as the rounding cut-off that gives the maximum sum of sensitivity and specificity. This value should be the shoulder at the top left of the ROC (receiver operating characteristic curve). Bootstrapping techniques were employed to validate the results. A logistic regression analysis was carried out to evaluate the influence of the severity of preoperative regurgitation on recurrence of moderate/severe MR. Cumulative probability for death and reoperation were estimated by the Kaplan-Meier method. SPSS 12.0 (SPSS, Chicago, IL, USA) and Stats Direct 2.5.7 (StatsDirect, Sale, UK) were used for these calculations. Significance for hypothesis testing was set at the 0.05 two tailed level.

\section{RESULTS}

\section{Left Ventricular Reverse Remodeling}

Table 1 displays perioperative data. No difference was detected between Groups.

In responders (Figure $1 \mathrm{~A}$ ), the mean end-systolic volume Index decreased significantly at discharge $(p<0.001)$, early $(p<0.001)$ and late controls $(p<0.001)$. In contrast, in non-responders (Figure $1 \mathrm{~B}$ ) ESVI reduced significantly at discharge ( $p=0.01$ ), it was stable at early follow-up ( $p=n s)$ but increased again at late control $(p<0.001)$. When the degree of LV reverse remodeling was compared further in the two groups, ESVI was reduced $\geq 10 \%$ in ten $(8.3 \%),<10 \%$ in $83(69.2 \%)$, was relatively stable $( \pm 1 \%)$ in $15(12.5 \%)$, and enlarged in $12(10.0 \%)$ non-responders. Among responders, 79 (94.0\%) had a reduction in ESVI of $15 \% \leq 20 \%$ and five $(6.0 \%)$ a reduction $>20 \%$. LVRR was not influenced by the type of ring employed (Figure 2).

\section{Clinical Endpoints and MR}

Early clinical outcome did not differ between Groups (Table 2). At late follow up, NYHA functional class was greater in non-responders $(\mathrm{p}<0.001)$.

During the follow-up 15 patients died; cause of death in responders $(n=2)$ were: malignancy ( 55 months) sudden, unexplained death (22 months). Cause of death in responders ( $n=13)$ were: heart failure ( $n=3 ; 36,45$, and 54 months), myocardial infarction $(\mathrm{n}=2 ; 31$ and 42 months), arrhythmia $(\mathrm{n}=1 ; 59$ months)], multi-organ failure ( $n=1 ; 6$ months), respiratory failure ( $n=1 ; 14$ months), infection $(n=2 ; 3$ and 8 months), stroke ( $n=2 ; 12$ and 43 months) and malignancy ( $n=1 ; 59$ months). 
Table 1. Perioperative data $(n=204)$

\begin{tabular}{|c|c|c|c|}
\hline & $\begin{array}{l}\text { Responders } \\
\mathrm{n}=84\end{array}$ & $\begin{array}{l}\text { Non-Responders } \\
\mathrm{n}=120\end{array}$ & $\mathbf{p}$ \\
\hline Age (years) & $68 \pm 7.4$ & $67 \pm 7.6$ & 0.26 \\
\hline Gender M/F & $51 / 33(60.7 / 39.3)$ & $78 / 42(65.0 / 35.0)$ & 0.55 \\
\hline NYHA class & $3[3-4]$ & $3[3-4]$ & 0.72 \\
\hline Angina & $40(47.6)$ & $54(45.0)$ & 0.69 \\
\hline $\mathrm{CCS}$ angina class & $2[1-3]$ & $2[1-3]$ & $>0.9$ \\
\hline \multicolumn{4}{|l|}{ Euroscore } \\
\hline Additive & $8.0[5-9]$ & $8.2[5-8]$ & 0.37 \\
\hline Logistic & $14.8[11-18]$ & $15.1[10-18]$ & 0.8 \\
\hline Hypertension & $32(38.1)$ & $48(40.0)$ & 0.86 \\
\hline Diabetes & $24(28.6)$ & $34(28.3)$ & 0.9 \\
\hline COPD & $12(14.3)$ & $17(14.2)$ & 0.9 \\
\hline Chronic renal disease & $13(15.5)$ & $19(15.8)$ & 0.9 \\
\hline Cerebral vascular disease & $11(13.1)$ & $18(15.0)$ & 0.51 \\
\hline Peripheral vascular disease & $6(7.1)$ & $6(5.0)$ & 0.3 \\
\hline Familiar history & $40(47.6)$ & $60(50.0)$ & 0.8 \\
\hline \multicolumn{4}{|l|}{ Myocardial infarction } \\
\hline$>3$ months & $62(73.8)$ & $99(82.5)$ & 0.15 \\
\hline$\leq 3$ months & $22(26.2)$ & $21(17.5)$ & 0.12 \\
\hline Inferior/Posterior & $40(47.7)$ & $60(50.0)$ & 0.77 \\
\hline Anterior/Septal & $7(8.3)$ & $12(10.0)$ & 0.53 \\
\hline Lateral & $18(21.4)$ & $24(20.0)$ & 0.8 \\
\hline Combined & $19(22.6)$ & $24(20.0)$ & 0.8 \\
\hline Coronary vessel disease & $2[1-3]$ & $2[1-3]$ & 0.8 \\
\hline Left Main & $14(16.7)$ & $21(17.5)$ & 0.66 \\
\hline \multicolumn{4}{|l|}{ Medications } \\
\hline $\begin{array}{l}\text { Angiotensin-converting enzyme } \\
\text { inhibitors }\end{array}$ & $66(78.6)$ & $100(83.3)$ & 0.46 \\
\hline$\beta$-adrenergic blockers & $46(54.8)$ & $62(51.7)$ & 0.56 \\
\hline Long-acting nitrates & $42(50.0)$ & $54(45.0)$ & 0.14 \\
\hline Diuretics & $71(84.5)$ & $105(87.5)$ & 0.32 \\
\hline Digitalis & $18(21.4)$ & $28(23.3)$ & 0.8 \\
\hline Calcium antagonișts & $12(14.3)$ & $20(16.7)$ & 0.7 \\
\hline Preoperative IABP & $3(3.6)$ & $5(4.2)$ & 0.8 \\
\hline \multicolumn{4}{|l|}{ Surgery } \\
\hline \multicolumn{4}{|l|}{ Cardioplegia } \\
\hline Antegrade & $76(90.5)$ & $106(88.3)$ & 0.06 \\
\hline Antegrade and Retrograde & $8(9.5)$ & $14(11.7)$ & 0.08 \\
\hline $\mathrm{CPB}$ time (min) & $118 \pm 28$ & $128 \pm 34$ & 0.06 \\
\hline CCL time (min) & $88 \pm 18$ & $96 \pm 19$ & 0.1 \\
\hline
\end{tabular}




\begin{tabular}{llll}
\hline & $\begin{array}{l}\text { Responders } \\
\mathbf{n = 8 4}\end{array}$ & $\begin{array}{l}\text { Non-Responders } \\
\mathbf{n = 1 2 0}\end{array}$ & $\mathrm{p}$ \\
\hline Surgical Approach & & & \\
$\quad$ Left atriotomy & $70(83.3)$ & $101(84.2)$ & 0.6 \\
Transseptal & $8(9.5)$ & $10(8.3)$ & 0.4 \\
$\quad \begin{array}{l}\text { Transseptal extended to LA roof } \\
\text { CABG }\end{array}$ & $6(7.2)$ & $9(7.5)$ & $>0.9$ \\
$\quad$ Anastomoses/patient & $2[1-3]$ & $2[1-2]$ & 0.7 \\
Arterial graft/patient & $1[1-2]$ & $1[1-2]$ & $>0.9$ \\
\hline
\end{tabular}

Continuous variables are presented as mean \pm standard deviation; Discrete variables are presented as percentage. Non-parametric variables were presented as median [Interquartile range].

Abbreviations: $\mathrm{M} / \mathrm{F}=\mathrm{Male} / \mathrm{Female}$;YHA=New York Heart Association; CCS=Canadian Cardiovascular Society; $\mathrm{COPD}=\mathrm{Chronic}$ obstructive pulmonary disease; IABP=Intaaortic balloon pump; $\mathrm{CPB}=\mathrm{Cardiopulmonary}$ bypass; $\mathrm{CCL}=$ (Aortic) Cross-clamp; $\mathrm{MR}=$ Mitral regurgitation;. $\mathrm{CABG}=$ Coronary artery bypass grafting.
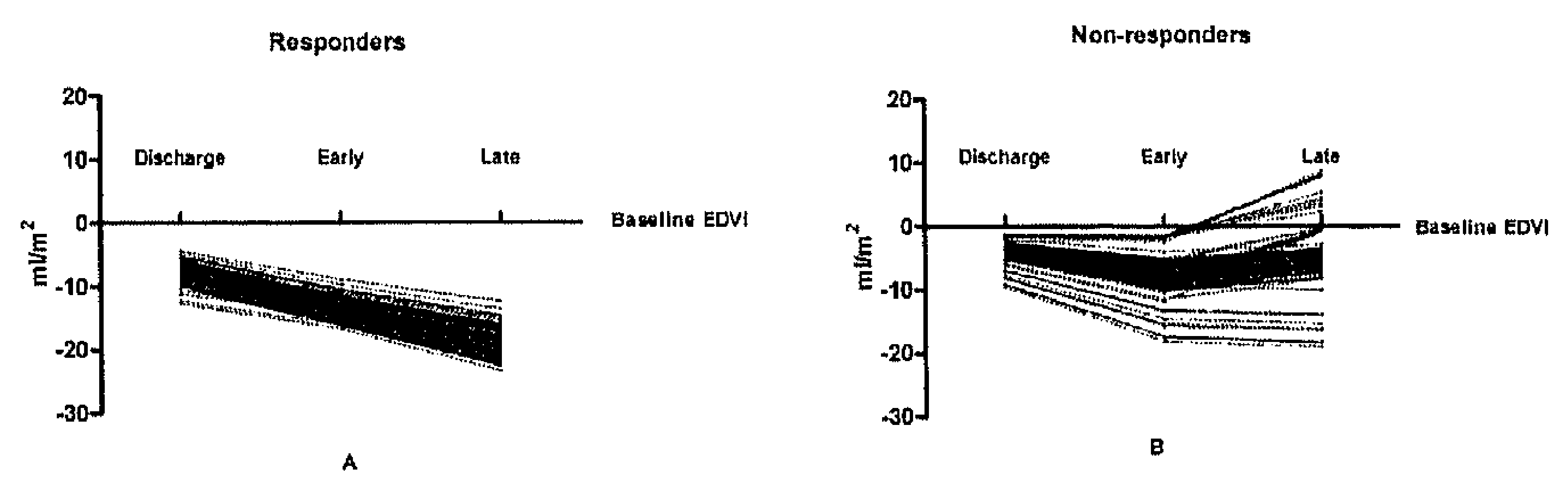

Figure 1. Reduction in end systolic volume Index in responders (A) and non-responders (B) of left ventricular reverse remodeling. See text.

Overall, five-year actuarial survival was $83.2 \pm 4.4$. It was significantly lower in non responders (Figure 3).

Data of mitral regurgitation are shown in Table 3. In responders, jet direction was posterior in the major part of patients whereas it was central or anterior in most of non-responders. In addition, $\mathrm{TA}(\mathrm{p}<0.001)$, and $\mathrm{CH}(\mathrm{p}=0.02)$ were preoperatively higher and CL $(\mathrm{p}=0.04)$ was shorter in non responders. No further significant difference between the two Groups was found regarding qualitative and quantitative MR data. At early control, the incidence of recurrent MR was $2.4 \%$ and $43.9 \%$ in responders and non-responders, respectively $(\mathrm{p}<0.001)$. At late control, recurrent $M R$ was unchanged in responders, whereas showed a further increment in non-responders $(67.3 \%, \mathrm{p}<0.001$ vs. responders). Whereas none of non responders showed a late recurrent $M R \geq 4,18(15 \%)$ non-responders experienced moderate/severe recurrent MR. Interestingly, in the latter Group, among 28 patients with severe (4+) preoperative MR, seven (25\%) developed severe and eleven (39.2\%) moderate recurrent 


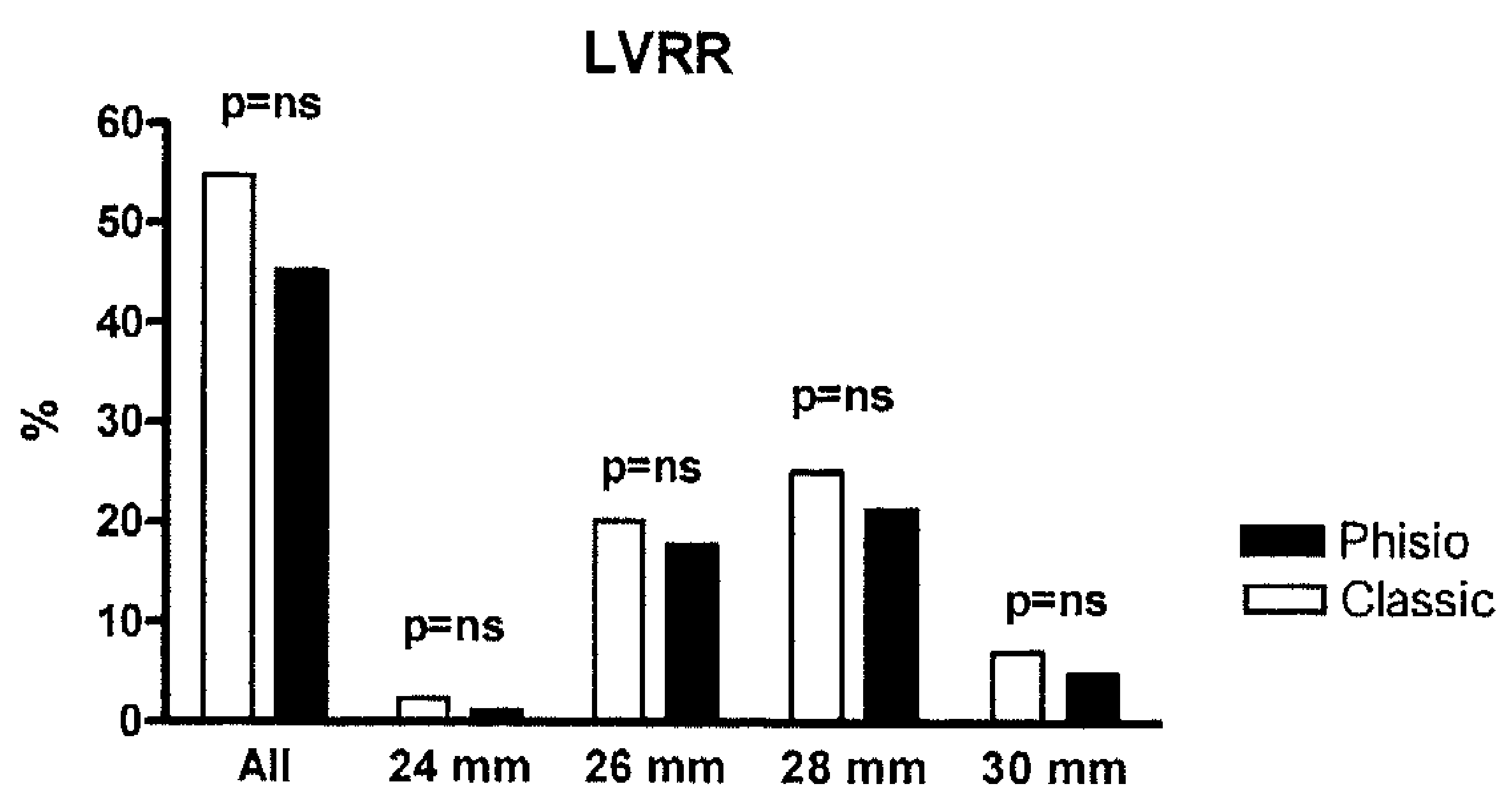

Figure 2. Left ventricular reverse remodeling (LVRR) by ring type/size. The extent of remodeling was not significantly different between rings at different sizes.

MR. At logistic regression analysis, MR grade $>3$ was a predictive factor of recurrent moderate/severe MR (OR=5.2 [95\% bias-corrected CI 24-6.3]; $\mathrm{p}<0.001)$. Among responders none underwent reoperation for failed repair. Contrastingly, four (3.7\%) non responders had a re-MV repair and $13(12.1 \%)$ underwent mitral valve replacement. Five-year freedom from reoperation for failed repair were $100 \%$ and $44 \pm 7 \%$ in responders and non responders, respectively $(\mathrm{p}<0.001)$.

Finally, three $(3.7 \%)$ non responders experienced cardiac-related events during the follow-up period $(\mathrm{p}<0.001$ vs. responders $[\mathrm{n}=0])$.

\section{Global and Local LV Remodeling}

Changes in end-diastolic volume Index and WMSI mirrored those of ESVI (Table 4). Left ventricular dimensions decreased, in responders, at any study point $(p<0.001)$. In contrast, for non-responders, there was an initial reduction in LV dimensions at discharge $(p=0.01)$ and early control $(p=0.02$ and $p=0.04$ for ESD and EDD, respectively), followed by a significant increase at late postoperative stage $(p<0.001)$. Ejection fraction $(p=0.001)$ and MPI $(p=0.01)$ significantly improved at late follow-up only in responders, whereas remained unchanged in non-responders at any study point. Systolic and diastolic sphericity indexes improved in responders at discharge $(p<0.001)$, early $\left(\mathrm{SI}_{S}, p=0.02\right.$ and $\mathrm{SI}_{\mathrm{D}}, p=0.03$, respectively) and late controls $(p<0.001)$. In non-responders, there was an initial but not significant reduction in both systolic and diastolic sphericity indexes, with a late increase exceeding the preoperative value $(p<0.001)$.

Non-responders had greater preoperative APM lateral and APM posterior, wider $\mathrm{PM}_{\mathrm{S}}$ separation and larger APM WMSI. Contrastingly, PPM WMSI was higher in 
Table 2. Clinical outcome $(n=204)$

\begin{tabular}{|c|c|c|c|}
\hline & $\begin{array}{l}\text { Responders } \\
\mathrm{n}=84\end{array}$ & $\begin{array}{l}\text { Non-Responders } \\
\mathrm{n}=120\end{array}$ & $\mathrm{p}$ \\
\hline ICU LoS (h) & $22[13-34]$ & $26[16-40]$ & 0.5 \\
\hline Time of MV (h) & $9[6-11]$ & $11[5-16]$ & 0.08 \\
\hline Inotropes & $7(8.3)$ & $11(9.2)$ & 0.8 \\
\hline Blood transfusion (Units) & $5[1-3]$ & $3[0-2]$ & 0.09 \\
\hline \multicolumn{4}{|l|}{ Early Complications } \\
\hline Rate & 28.6 & 36.7 & 0.09 \\
\hline Bleeding & $4(4.8)$ & $8(6.7)$ & \\
\hline Low-output syndrome & $1(1.2)$ & $2(1.7)$ & \\
\hline IABP & $1(1.2)$ & $2(1.7)$ & \\
\hline MI & $1(1.2)$ & $3(2.5)$ & \\
\hline $\mathrm{AF}$ & $8(9.5)$ & $13(10.8)$ & \\
\hline Permanent pacemaker & $1(1.2)$ & $1(0.8)$ & \\
\hline Renal failure & $1(1.2)$ & $I(0.8)$ & \\
\hline Respiratory failure & $3(3.6)$ & $7(5.8)$ & \\
\hline CVA & - & $1(0.8)$ & \\
\hline Infection & $3(3.6)$ & $6(5.0)$ & \\
\hline In-hospital LoS (days) & $7[5-8]$ & $7[4-9]$ & $>0.9$ \\
\hline NYHA class & $2[1-2]$ & $3[2-3]$ & $<0.001$ \\
\hline 1 & $38(45.2)$ & $5(4.1)$ & \\
\hline 2 & $39(46.4)$ & $32(26.7)$ & \\
\hline 3 & $7(8.3)$ & $63(52.5)$ & \\
\hline 4 & - & $20(16.7)$ & \\
\hline \multicolumn{4}{|l|}{ Late Complications } \\
\hline Rate & 4.8 & 2.5 & 0.38 \\
\hline Endocarditis & $1(1.2)$ & - & \\
\hline CVA & - & $1(0.8)$ & \\
\hline Others & $3(3.6)$ & $2(1.7)$ & \\
\hline
\end{tabular}

Discrete variables are presented as percentage (parentheses). Non-parametric data are shown as median [Interquartile range].

Abbreviations: ICU=Intensive Care Unit; LoS=Length of Stay; $M V=$ Mechanical Ventilation (time of). IABP=Intaaortic balloon pump; MI=Myocardial infarction; $A F=$ Atrial Fibrillation; $\mathrm{CVA}=$ Cerebrovascular accident documented by Computed Tomography; NYHA=New York Heart Association. 


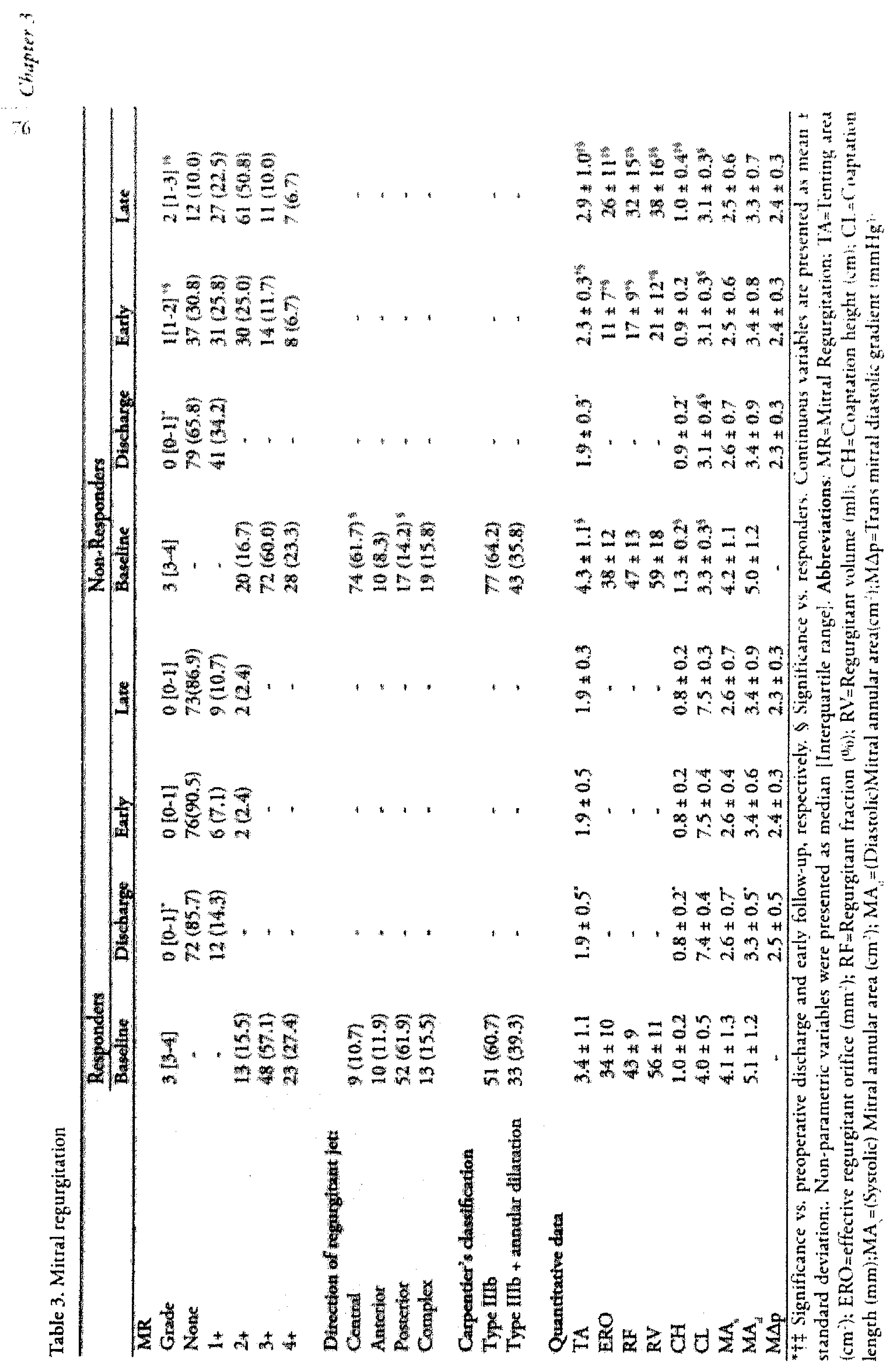




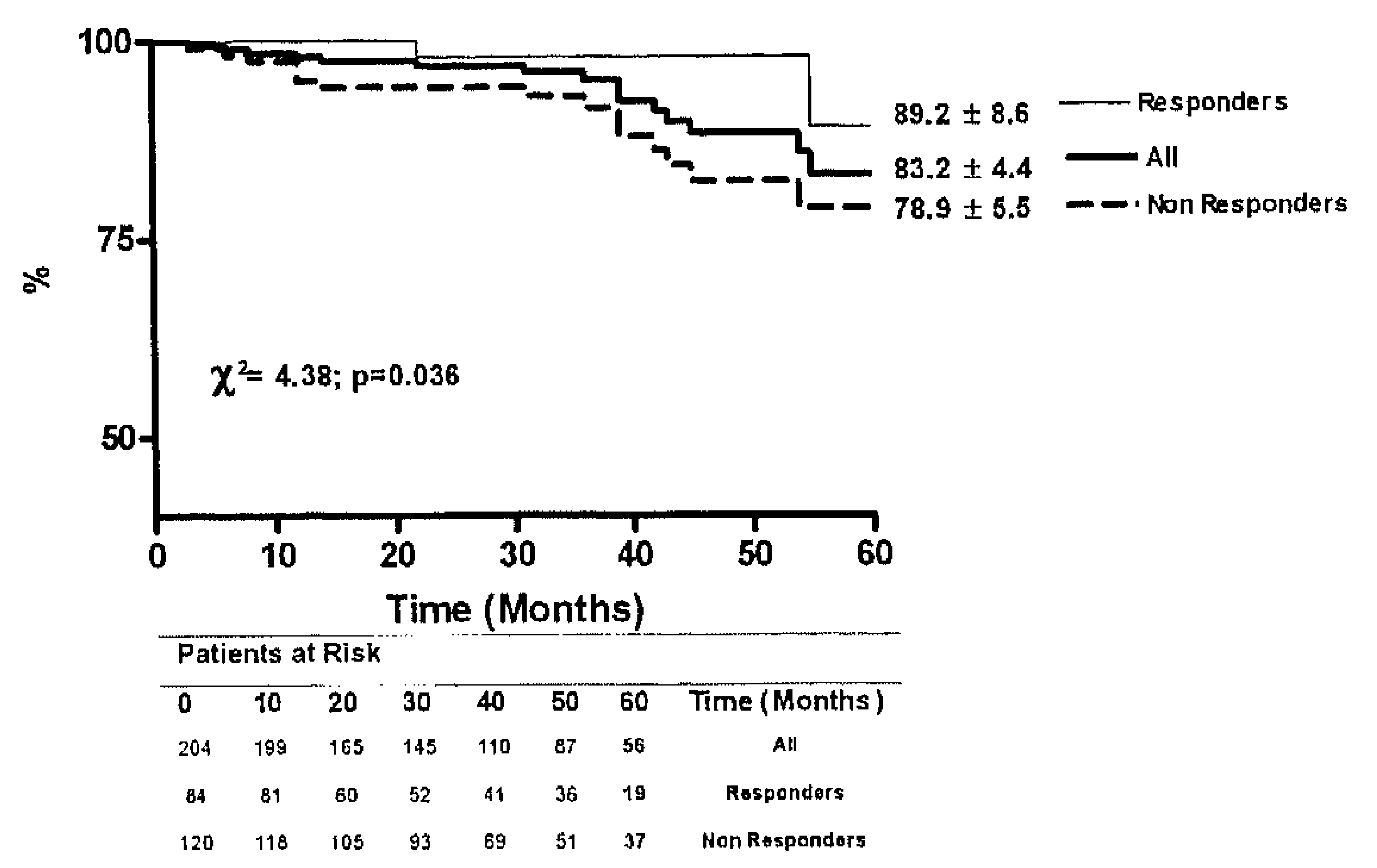

Figure 3. Five-year actuarial Survival by Groups. See text.

responders, whereas PPM posterior, PPM lateral and PPM fibrosis were comparable in the two Groups.

At discharge, in responders, indexes of posterior and lateral displacement of papillary muscles, $\mathrm{PM}_{s}$ separation, PPM fibrosis and WMSIs were reduced significantly. All these index did not show any significant change afterwards. In nonresponders, all the above indexes (except for APM WMSI which remained constant over time) showed a reduction, even not significant, at discharge, remained constant at early control showing a significant late increase.

\section{PREDICTORS OF LVRR}

At univariable analysis (Table 4), left ventricular diameters, sphericity indexes, LVEF, WMSI, MPI, APM posterior and lateral, PM separation and APM WMSI were predictors of LVRR. By multiple regression analysis (Table 6) baseline MPI, SI and WMSI resulted as independent predictors of LVRR.

Internal validation of such multivariable analysis by means of bootstrapping, including the same variables of standard logistic regression model, further confirmed the significant predictive of recurrent MR (bootstrap OR=2.4, 95\% bias-corrected 95\% CI=1.1-5.3). Finally, logistic regression model was reliable (Hosmer-Lemeshow test, $\mathrm{p}=0.6$ ) and accurate ( $\mathrm{c}$ index $=0.8$ ). ROC curve analysis to determine optimal cutoff values to predict reverse $\mathrm{LV}$ (Figures 4-6) showed that $\mathrm{SI}_{S}$ had $90 \%$ of sensitivity and specificity with an optimal cutoff $<0.7$ (95\% CI by bootstrapping 


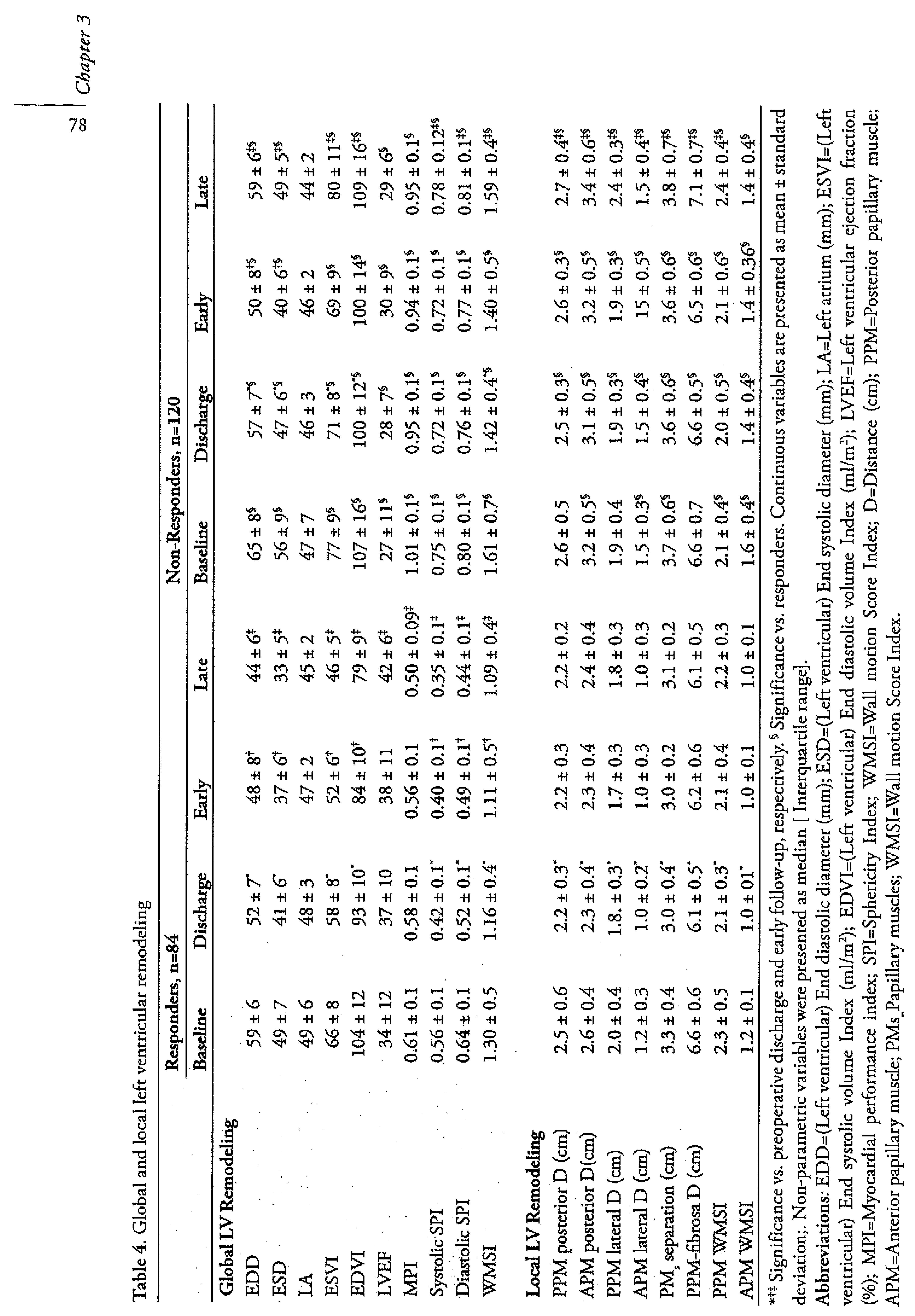


Table 5. Univariable predictors of LVRR

\begin{tabular}{|c|c|c|c|}
\hline Variable & Odds Ratio & $95 \% \mathrm{CI}$ & $p$ \\
\hline MPI & 5.82 & $1.84-7.26$ & $<0.001$ \\
\hline $\mathrm{SI}_{\mathrm{S}}$ & 9.51 & $4.60-12.4$ & $<0.001$ \\
\hline WMSI & 3.04 & $0.52-3.59$ & 0.001 \\
\hline APM posterior $D$ & 3.19 & $1.55-6.55$ & 0.006 \\
\hline ESD & 2.92 & $1.12-6.01$ & 0.01 \\
\hline APM WMSI & 3.82 & $1.84-7.26$ & 0.01 \\
\hline $\mathrm{PM}_{s}$ separation & 2.32 & $1.30-3.85$ & 0.02 \\
\hline EDD & 2.48 & $1.38-4.41$ & 0.03 \\
\hline LVEF & 2.56 & $1.22-5.33$ & 0.03 \\
\hline$S I_{D}$ & 2.49 & $1.34-4.63$ & 0.03 \\
\hline APM lateral D & 2.63 & $1.11-3.40$ & 0.04 \\
\hline MI localizzation & 2.05 & $1.01-4.18$ & 0.06 \\
\hline ESWS & 1.66 & $0.79-3.48$ & 0.15 \\
\hline Direction of jet & 1.05 & $1.0-3.18$ & 0.16 \\
\hline $\mathrm{CCS}$ angina class & 0.55 & $0.27-1.13$ & 0.21 \\
\hline Chronic renal disease & 0.19 & $0.14-1.88$ & 0.36 \\
\hline Cerebral vascular disease & 0.64 & $0.24-1.56$ & 0.36 \\
\hline COPD & 1.41 & $0.60-3.01$ & 0.37 \\
\hline Gender & 1.72 & $0.49-5.76$ & 0.38 \\
\hline MI $<3$ months & 1.50 & $0.51-3.49$ & 0.45 \\
\hline Number of diseased coronary arteries & 1.44 & $0.50-5.12$ & 0.47 \\
\hline NYHA class & 1.28 & $0.62-1.62$ & 0.48 \\
\hline Diseased left main coronary artery & 1.55 & $0.85-2.81$ & 0.52 \\
\hline Number of graft & 1.29 & $0.57-2.64$ & 0.53 \\
\hline Arterial graft & 1.25 & $0.56-2.78$ & 0.53 \\
\hline Age (years) & 1.24 & $0.58-2.64$ & 0.56 \\
\hline Smoking & 0.58 & $0.10-2.51$ & 0.56 \\
\hline Euroscore & 1.32 & $0.44-3.50$ & 0.57 \\
\hline Hypertension & 0.61 & $0.10-2.62$ & 0.6 \\
\hline Diabetes & 1.38 & $0.42-4.65$ & 0.61 \\
\hline Peripheral vascular disease & 0.74 & $0.13-2.96$ & 0.61 \\
\hline Familiar history & 0.76 & $0.25-2.03$ & 0.62 \\
\hline CCL time & 1.22 & $0.48-2.91$ & 0.61 \\
\hline CPB time & 1.31 & $0.44-3.15$ & 0.62 \\
\hline ERO & 1.33 & $0.30-5.13$ & 0.62 \\
\hline PPM - fibrosa D & 0.82 & $0.33-2.06$ & 0.63 \\
\hline PPM WMSI & 1.28 & $0.50-3.60$ & 0.64 \\
\hline $\mathrm{MR}$ & 1.51 & $0.27-4.61$ & 0.65 \\
\hline $\mathrm{TA}$ & 0.78 & $0.26-2.01$ & 0.73 \\
\hline Leaflet tethering & 0.9 & $0.46-2.31$ & 0.8 \\
\hline Preoperative medications & 1.76 & $0.30-3.42$ & 0.8 \\
\hline RF & 1.27 & $0.26-3.57$ & 0.8 \\
\hline
\end{tabular}




\begin{tabular}{llll}
\hline Variable & Odds Ratio & $95 \% \mathrm{CI}$ & $\mathrm{P}$ \\
\hline RV & 1.09 & $0.52-2.27$ & 0.82 \\
Ring type & 0.93 & $0.42-2.02$ & 0.85 \\
Ring size & 0.94 & $0.59-1.50$ & 0.82 \\
LA & 0.94 & $0.31-2.40$ & 0.91 \\
PPM posterior D & 1.03 & $0.49-2.33$ & 0.93 \\
PPM lateral D & 0.94 & $0.31-2.40$ & 0.91 \\
\hline
\end{tabular}

Abbreviations: LVRR=Left ventricular reverse remodeling; NYHA=New York Heart Associa-

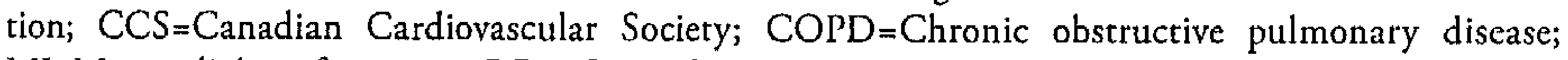
$\mathrm{MI}=$ Myocardial infarction; $\mathrm{CCL}=$ Cross-clamp; $\mathrm{CPB}=\mathrm{Cardiopulmonary}$ bypass; $\mathrm{MR}=(\mathrm{Grade}$ of preoperative) Mitral regurgitation; $T A=$ Tenting area; $E R O=$ effective regurgitant orifice; $\mathrm{RF}=$ Regurgitant fraction; $\mathrm{RV}=$ Regurgitant volume; $\mathrm{EDD}=\mathrm{End}$ diastolic diameter; $\mathrm{ESD}=$ End systolic diameter ; LA=Left atrium; LVEF=Left ventricular ejection fraction (\%); MPI=Myocardial performance index; SIS=Systolic sphericity Index; SID=Diastolic sphericity Index; WMSI=Wall motion Score Index; ESWS=End systolic wall stress; $E=P$ eak trans-mitral flow velocity; $A=$ =eak late trans-mitral flow velocity; DT=Deceleration time at early diastolic filling $(\mathrm{ms})$; IVRT=Isovolumetric relaxation time; PPM=Posterior papillary muscle; $\mathrm{D}=\mathrm{D}$ istance; $\mathrm{APM}=$ Anterior papillary muscle; PMs=Papillary muscles; WMI=Wall motion index.

Table 6. Logistic regression for LVRR

\begin{tabular}{|c|c|c|c|c|}
\hline & & & \multicolumn{2}{|c|}{ Bias corrected $95 \% \mathrm{CI}$} \\
\hline Variable & Odds Ratio & $\mathrm{p}$ & Lower Limit & Upper Limit \\
\hline $\mathrm{SI}_{\mathrm{s}}$ & 4.918 & $<0.001$ & 2.107 & 5.993 \\
\hline MPI & 4.753 & $<0.001$ & 2.012 & 5.463 \\
\hline WMSI & 2.612 & 0.003 & 0.903 & 3.841 \\
\hline APM posterior & 1.625 & 0.06 & 0.871 & 2.433 \\
\hline$S I_{D}$ & 1.670 & 0.09 & 0.934 & 2.981 \\
\hline LVEF & 1.612 & 0.1 & 0.954 & 2.666 \\
\hline APM WMSI & 1.323 & 0.13 & 0.925 & 3.649 \\
\hline ESD & 1.472 & 0.115 & 0.091 & 2.231 \\
\hline APM lateral & 0.980 & 0.2 & 0.091 & 2.033 \\
\hline $\mathrm{EDD}$ & 0.932 & 0.247 & 0.081 & 1.789 \\
\hline $\mathrm{PM}_{\text {, separation }}$ & 1.01 & 0.3 & 0.096 & 2.012 \\
\hline
\end{tabular}

Abbreviations: LVRR=Left ventricular reverse remodeling; EDD=End diastolic diameter; $E S D=E n d$ systolic diameter; LVEF=Left ventricular ejection fraction; MPI=Myocardial performance index; $\mathrm{SI}_{\mathrm{SS}}$ Systolic sphericity Index; $\mathrm{SI}_{\mathrm{D}}=$ Diastolic sphericity Index; WMSI=Wall motion Score Index; $\mathrm{DT}=$ Deceleration time at early diastolic filling. 
$0.58-0.81)$. MPI with a cutoff $<0.9$ (95\% CI by bootstrapping $0.75-0.96)$ had $100 \%$ of sensitivity and specificity. Finally, WMSI $<1.5$ (95\% CI by bootstrapping $1.35-$ 1.62) had $80 \%$ of sensitivity and $82 \%$ of specificity.

\section{COMMENT}

The most common surgical technique for CIMR is undersized mitral ring annuloplasty (UMRA) ${ }^{7}$. Although this procedure does not fully address the ventricular causes of CIMR, it is simple to perform and reproducible, and therefore has been widely adopted worldwide by cardiac surgeons. Nonetheless, after encouraging initial results ${ }^{8}$, different studies have revealed a significant proportion of patients developing recurrent $M R$ during follow-up ${ }^{6,10}$ which has been mainly related to continued LV remodeling and persistent leaflet tethering, ${ }^{9,30}$. Thus left ventricular reverse geometric remodeling (LVRR) is an important aim in the surgical treatment of CIMR.

Based on the same criterion used by Stellbrink et $\mathrm{al}^{16}$, in our study nonresponders were observed in $58.8 \%$ of the study population which included $10 \%$ with further increase in ESVI. Braun and coworkers ${ }^{31}$ demonstrated $39.5 \%$ of non responders among 87 consecutive patients with CIMR undergoing restrictive mitral annuloplasty, considering a $10 \%$ reduction in LV end diastolic dimension as significant LVRR. However, measurements of LV volumes have been demonstrated to be more reliable than LV diameters in assessing LV remodeling, especially in enlarged ventricles ${ }^{32}$.

In our experience, non-responders had a higher postoperative NYHA class $(p<0.001)$, higher recurrence of mitral regurgitation $(p<0.001)$ and higher reoperation rate for failed repair $(p<0.001)$. In addition, 5 -year survival was significantly lower in responders.

By comparing the two groups in our study, it appears evident that non-responders were preoperatively much more globally remodeled and that at late control they still showed more spherical, enlarged and dysfunctional left ventricle. Furthermore, the late increase in sphericity indexes in non-responders, exceeding the preoperative value, was consistent with further remodeling after UMRA, and reflects increased tethering on the mitral valve and greater displacement of $\mathrm{PM}_{s}$. Furthermore, in our experience, non-responders had preoperatively a greater lateral and posterior displacement of the APM and a wider $\mathrm{PM}_{S}$ separation. Previous studies ${ }^{11,33}$ demonstrated that the local remodeling of the LV segments supporting PPM is a necessary condition for the development of MR and, accordingly, we found that PPM displacement occurred preoperatively in both Groups even, in non-responders, lateral and posterior displacement of APM were predominant.

In addition this Group had larger WMSI of the segments underlying the APM whereas PPM WMSI was higher in responders. These results support previous clin- 

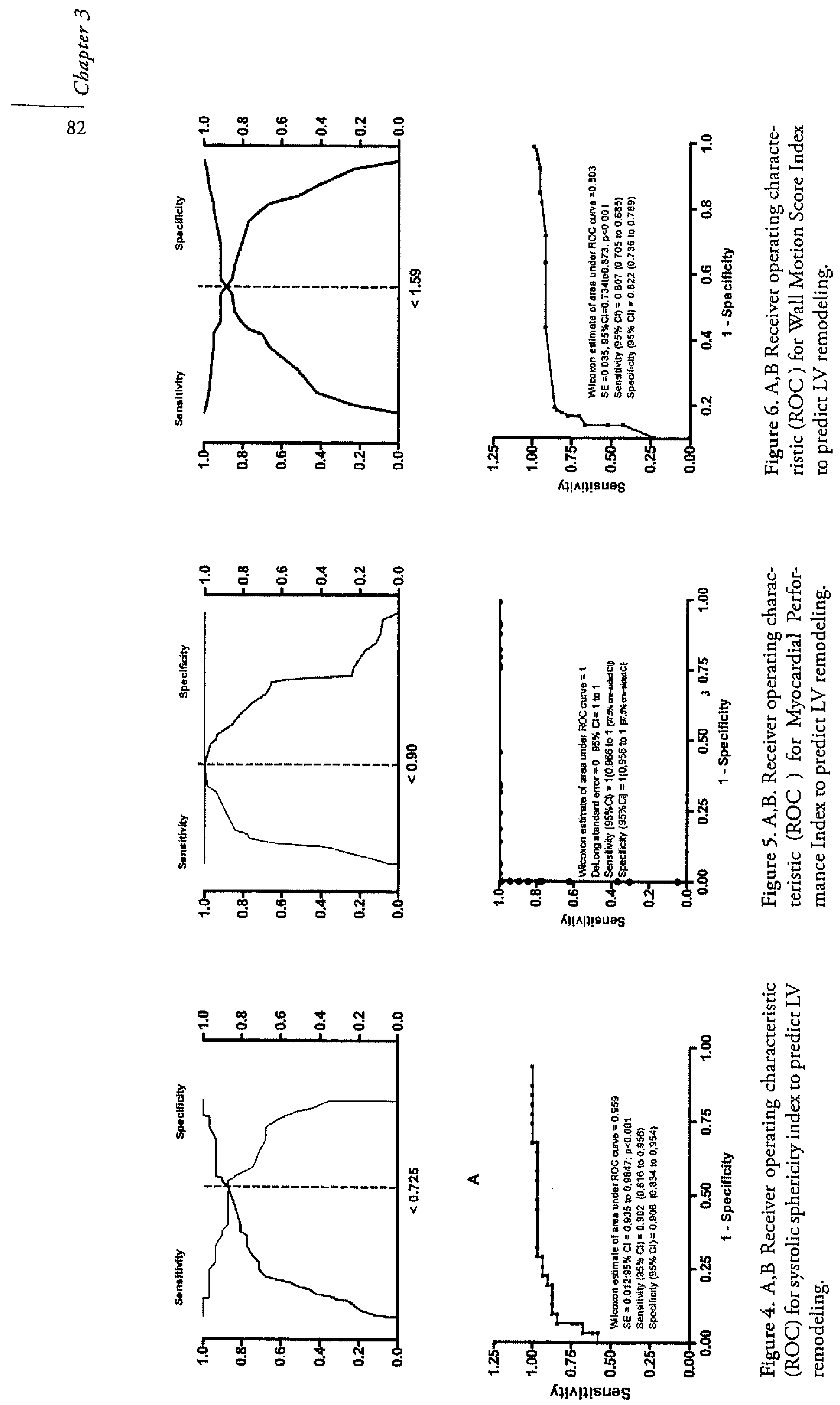
ical findings reported by Agricola et a ${ }^{34}$ who described two Groups of patients with CIMR according to tethering pattern. Likewise non-responder-patients in our study, their symmetric Group had prevalent posterior and lateral displacement of APM (both $\mathrm{p}<0.04$ ), PMs separation and APM WMSI (both<0.0001).

Interestingly, this different distribution of local wall abnormalities did not reflect, in our experience, a different infarct localization and we cannot exclude that this different pattern of regional functional abnormalities is caused by progressive deterioration in contractile function with progressive recruitment of border zone myocardium into scar in patients with more dysfunctional LVs. Thus, the predominant displacement of APM and the larger APM WMSI might represent a sign of disease progression. Nonetheless, in our study, this pattern clearly identified subjects which are expected not to reverse-remodel after annuloplasty.

It would have been helpful to assess the regional viability of the regions associated with the anterior and posterior papillary muscles to confirm these findings.

After surgery, we showed that continued LV local remodeling occurs and it predominantly involves the region of the LV supporting the posterior papillary muscle whereas APM WMSI remained constant over time in both Groups.

Continued LV remodeling increases leaflets tethering leading to progressive lack of coaptation. In this setting the coaptation length gained at the end of procedure could play a significant role in preventing MR recurrence. The coaptation length should be sufficient to balance tethering forces secondary to postoperative remodeling thus another reason for these results might be the acceptance of a leaflet coaptation of only $5 \mathrm{~mm}$ at the end of the procedure which could not be adequate for all patients. Indeed, non responders showed a $\mathrm{CL}<5 \mathrm{~mm}$ at any postoperative control and this value was significantly lower than responders $(p<0.001)$ even with comparable ring size between Groups ( $\mathrm{p}=\mathrm{ns}$ ).

Thus, actually we believe that, in subjects who are expected not to reverse remodel, a minimum CL of $8 \mathrm{~mm}$ should be achieved ${ }^{31}$. Contrastingly, in patient with strongly tethered AML or with less tethered anterior leaflet but which is not sufficiently long to ensure a proper postoperative CL, regurgitation cannot be eliminated by ring annuloplasty. In these patients a chordal sparing mitral valve replacement technique should be considered.

A key point for achieving an appropriate leaflet coaptation and to ensure durable results is a true undersizing and, in this setting, a pivotal role can be played by the type of ring chosen. In the present experience we used only two rings (Carpentier's rigid or Physio semi-flexible rings). These two rings are not at all identical, thus, even we have undersized by two sizes both rings, we might have been less restrictive with the Physio ring than with the Classic ring. However, in our experience, the extent of LVRR was comparable between rings at any size. Furthermore neither ring type nor ring size were predictive of LVRR at logistic regression analysis. 
Additionally, fifteen percent of non-responders experienced moderate/severe recurrent MR. Of interest, $64.2 \%$ of non responders with preoperative severe (4+) $M R$, developed a late recurrent $M R \geq 3+$ and preoperative $M R$ grade $>3$ was a predictive factor of recurrent moderate/severe $M R(p<0.001)$. This finding deserve further investigation as these subjects might be not ideal candidates for UMRA and could more benefit from a chordal sparing mitral valve replacement.

Finally, at multivariable model, SIs $<0.72, \mathrm{MPI}<0.90$ and WMSI $<1.59$ resulted independent predictors of LVRR. These results emphasize the importance of the extent of preoperative LV global remodeling as having a central role in predicting inadequate results after UMRA. Furthermore, these findings confirm that the increase of sphericity index at end of systole predicts recurrence of MR after restrictive annuloplasty'. A new finding in this study is that MPI predicts the likelihood of reverse remodeling, whereas LVEF was not significant. The myocardial performance Index is a simply measurable Doppler-derived index of combined systolic and diastolic performance which, differently from LVEF, is independent of LV loading conditions and heart rate ${ }^{19}$. It is the summation of isovolumetric contraction and relaxation time divided by the ejection time. MPI has a narrow range of values in subjects with normal left ventricular function $(0.37 \pm 5)$. Ventricular dysfunction prolongs isovolumetric relaxation and shortens ejection times, resulting in an increase of the index compared with normal subjects.

\section{Study Limitations}

Our study findings should be viewed in light of some inherent limitations.

- Evaluation of LVRR was based on volumes obtained by echocardiography; Volumetry by $2 \mathrm{D}$ echocardiography depends on geometric assumptions and is subject to image-plane positioning errors. Hence it is not accurate in shape distorted postinfarction left ventricles. However, this limitation belongs to most of published paper regarding this pathology.

- Viability testing was not performed in these patients. Therefore lack of LVRR in non responders might be also due to irreversible ischemic myocardial damage (notviable myocardium). Furthermore, in relation to the papillary muscle displacement data presented, the revascularization of viable regions related to papillary muscle function might be the most important predictor of success with this operation. This issue deserves further investigation.

- Postoperative evaluation of the coronary status was not assessed. It would have been helpful to distinguish surgical failure from coronary disease progression.

- The issue of annulus re-shaping during annuloplasty has not been addressed. We employed only rigid/semi-flexible plane annuloplasty rings which may flatten the natural saddle shape causing greater tension on chordae and leaflets. Innovative 
annular rings mimicking the shape of healthy mitral annulus have been recently introduced in the market ${ }^{35-37}$.

- Estimated cutoffs are known to be very susceptible to changes in the study population.

We employed bootstrapping techniques to validate the results, nonetheless, it has also been documented that the sensitivity/specificity associated with these cutoffs are overly optimistic.

\section{STRENGTH OF THE STUDY}

This is a large cohort reported with UMRA in CIMR patients with detailed (100\% complete) echocardiographic follow-up. Furthermore our patient cohort was more homogeneous than in other study ${ }^{38}$ : all patients underwent associated CABG, they had no concomitant $\mathrm{MV}$ procedures, and the entire cohort was uniform regarding the MV ischemic leaflet dysfunction. Moreover, to assess results for MV repair, we studied true "recurrent" MR excluding those patients with "residual "MR in whom the insufficiency was presumably never eliminated at surgery. Additionally, we undertook valve sizing in a standardized fashion and the degree of undersizing was homogeneous over the 5-year-period of the study.

\section{Clinical Implications}

In our experience combined CABG and UMRA do not ensure successful and durable elimination of MR and significant LVRR in all patients. Those who can benefit from this approach could be preoperatively identified utilizing echo predictors. However, restrictive annuloplasty resulted to be ineffective in a large percentage of patients and results of this study suggest the need, in most of CIMR patients, for different approaches directly addressing ventricular tethering like second-order chordal cutting ${ }^{3}$, infarct placation ${ }^{4}$, papillary muscle sling 5 , papillary muscle imbrication $^{39}$, PPM surgical relocation ${ }^{40}$. Nonetheless, although these techniques appear promising, more data are required before they can be recommended for CIMR patients.

Additionally, the employment of support devices ${ }^{41,42}$ was introduced in the recent years in clinical practice, thus experiences with these techniques are actually in their preliminary phase.

Finally, mitral valve replacement, largely employed in the past in ischemic regurgitation, although eliminates the short-term risk of recurrent $M R$, is associated with poor long-term survival ${ }^{43}$. Thus, in our actual policy, we do not consider MV replacement as a reasonable alternative to repair in all patients. 
Our experience suggests that more information on possible echo predictors of an inadequate result may improve preoperative decision-making of CIMR patients for UMRA. 
1. Grigioni F, Enriquez-Sarano M, Zehr KJ, Bailey KL, Tajik AJ. Ischemic Mitral Regurgitation. Long-term outcome and prognostic implications with quantitative Doppler assessment. Circulation. 2001;103(13):1759-64.

2. Yiu SF, Enriquez-Sarano M, Tribouilloy C, Seward JB, Tajik AJ. Determinants of the degree of functional mitral regurgitarion in patients with systolic left vencricular dysfunction: a quantitative clinical study. Circulation. 2000;102(12):1400-6

3. Messas E, Guerrero JL, Handschumacher MD et al. Chordal cutting: a new therapeutic approach for ischemic mitral regurgitation. Circulation. 2001;104(16):1958-63.

4. Liel-Cohen N, Guerroro JL, Otsuji Y, et al. Design of a new surgical approach for ventricular remodeling to relieve ischemic mitral regurgitation: insights from three-dimensional echocardiography. Circulation 2000: 101:2756-63.

5. Hvass U, Tapia M, Baron F, Pouzet B Shafy A. Papillary muscle sling: a new functional approach to mitral repair in patients with ischemic left ventricular dysfunction and functional mitral regurgitation.Ann Thorac Surg 2003;75(3):809-11.

6. Tahta SA, Oury JH, Maxwell JM, Hiro SP, Duran CM. Outcome after mitral valve repair for functional ischemic mitral regurgitation. J Heart Valve Dis. 2002;11(1):11-8;

7. Bolling SF, Pagani FD, Deeb GM, Bach DS. Intermediate term outcome of mitral reconstruction in cardiomyopathy.J Thorac Cardiovasc Surg 1998;115(2):381- 6.

8. Bolling SF, Deeb GM, Bach DS.Mitral valve reconstruction in elderly, ischemic patients. Chest 1996;109(1):35- 40.

9. Bax JJ, Braun J, Somer ST et al.Restrictive annuloplasty and coronary revascularization in ischemic mitral regurgitation results in reverse left ventricular remodeling. Circulation. 2004;110(11 Suppl 1):II103-8.

10. McGee EC, Gillinov AM, Blackstone EH et al. Recurrent mitral regurgitation after annuloplasty for functional ischemic mitral regurgitation. J Thorac Cardiovasc Surg. 2004;128(6):916-24.

11. Hung J, Papakostas L, Tahta SA et al.Mechanism of recurrent ischemic mitral regurgitation after annuloplasty.Continued LV remodelling as a moving target. Circulation. 2004 Sep 14;110(11 Suppl 1):II85-90.

12. Geidel S, Lass $M, S$ chneider $C$ et al. Downsizing of the mitral valve and coronary revascularization in severe ischemic mitral regurgitation results in reverse left ventricular and left atrial remodeling.Eur J Cardiothorac Surg. 2005;27(6):1011-6.

13. World Medical Association Declaration of Helsinki: Ethical principles for medical research involving human subjects. Edinburgh, Scotland: World Medical Association, October 2000. Available at: http//www.wma.net/e/policy/17-c_3html. Accessed June 22, 2001.

14. Pfeffer MA, Braunwald E, Moye LA et al.Effect of captopril on mortality and morbidity in patients with left ventricular dysfunction after myocardial infarction. Results of the survival and ventricular enlargement trial. The SAVE investigators. N Eng1 J Med1992;327(10):669-77.

15. Carpentier A.Cardiac valve surgery-The "French correction". J Thorac Cardiovasc. Surg. 1983; 86: 326-37. 
16. Stellbrink C, Breithardt OA, Franke A et al.PATH-CHF (Pacing Therapies in Congestive Heart Failure) Investigators; CPI Guidant Congestive Heart Failure Research Group. Impact of cardiac resynchronization therapy using hemodynamically optimized pacing on left ventricular remodeling in patients with congestive heart failure and ventricular conduction disturbances.J Am Coll Cardiol. 2001;38(7):1957-65.

17. Bland MJ, Altman DJ.Staristical method for assessing agreement between two merhods of clinical measurements.Lancet 1986; 1:307-310.

18. Schiller NB, Shah PM, Crawford $M$ et al. Recommendations for quantitation of the left ventricle by two-dimensional echocardiography. American Society of Echocardiography Commitree on Standards, Subcommittee on Quantitation of Two-Dimensional Echocardiograms. J Am Soc Echocardiogr, 1989;2(5):358.

19. Tei C, Ling LH, Hodge DO et al. New index of combined systolic and diastolic myocardial performance: a simple and reproducible measure of cardiac function a study in normals and dilated cardiomyopathy. J Cardiol 1995; 26(6):357-66.

20. Kono T, Sabbah HN, Rosman H, Alam M, Jafri S, Goldstein S.Left ventricular shape is the primary determinant of functional mitral regurgitation in heart failure.J Am Coll Cardiol 1992;20(7):1594-8.

21. Cerqueira MD, Weissman NJ, Dilsizian Vet al. American Heart Association Writing Group on Myocardial Segmentation and Registration for Cardiac Imaging. Standardized myocardial segmentation and nomenclature for tomographic imaging of the heart: a statement for healthcare professionals from the Cardiac Imaging Committee of the Council on Clinical Cardiology of the American Hearr Association.Circulation. 2002;105(4):539-42.

22. Gardin JM, Adams DB, Douglas PS et al. American Society of Echocardiography. Recommendations for a standardized report for adult transthoracic echocardiography: a report from the American Society of Echocardiography's Nomenclature and Standards Committee and Task Force for a

23. Standardized Echocardiography Report. J Am Soc Echocardiogr. 2002;15(3):275-90

24. Zoghbi WA, Enriquez-Sarano M, Foster E et al. American Society of Echocardiography.Recommendations for evaluation of the severity of native valvular regurgitation with two-dimensional and Doppler echocardiography. J Am Soc Echocardiogr2003; 16(7):777-802

25. Boltwood CM, Tei C, Wong M, Shah PM. Quantitative echocardiography of the mitral complex in dilated cardiomyopathy: the mechanism of functional mitral regurgitation. Circulation 1983; 68(3):498-508

26. Vijayaraghavan G, Boltwood CM, Tei C, Wong M, Shah PM. Simplified echocardiographic measurement of the mitral annulus. Am Heart J 1986; 112(5):985-91.

27. Romesburg HC.Cluster analysis for researchers. North Carolina: Lulu Press; 2004.1-330.

28. Harrel FE, Lee KL, Califf RM, Pryor DB, Rosati RA. Regression modeling strategies for improved prognostic prediction. Statistics in Medicine 1984; 3:143-52.

29. Harrel FE Jr, Lee KL, Mark DB. Multivariable Prognostic Models:Issues in developing models, evaluating assumptions and adequacy, and measuring and reducing errors. Stats Med 1996:36187. 
30. Hosmer DW, Lemeshow S. Applied Logistic regression.2nd ed.New York, NY: Wiley-Interscience; 2000:143-202.

31. Kuwahara E, Orsuji $Y$, Iguro $Y$ et al. Mechanism of recurrent/persistent ischemic/functional mitral regurgitation in the chronic phase after surgical annuloplasty: importance of augmented posterior leaflet tethering. Circulation. 2006 Jul 4;114(1 Suppl):I529-34.

32. Braun J, Bax JJ, Versteegh MI et al.Preoperative left ventricular dimensions predict reverse remodeling following restrictive mitral annuloplasty in ischemic mitral regurgitation.Eur $\mathrm{J}$ Cardiothorac Surg. 2005;27(5):847-53.

33. Dujardin KS, Enriquez-Sarano M, Rossi A, Bailey KR, Seward JB. Echocardiographic assessment of left ventricular remodeling: are left ventricular diameters suitable tools? J Am Coll Cardiol. 1997 Nov 15;30(6):1534-41.

34. Gorman JH 3rd, Gorman RC, Plappert $T$ et al. Infarct size and location determine development of mitral regurgitation in the sheep model. J Thorac Cardiovasc Surg. 1998;115(3):615-22.

35. Agricola E, Oppizzi M, Maisano $F$ et al. Echocardiographic classification of chronic mitral regurgitation caused by restricted motion according to tethering pattern. Eur J Echocardiography 2004; 5: 326-34.

36. Votta E, Maisano F, Bolling SF, Alfieri O, Montevecchi FM, Redaelli A. The Geoform diseasespecific annuloplasty system: a finite element study. Ann Thorac Surg. 2007;84(1):92-101.

37. Salgo IS, Gorman JH, Gorman RC, et al. Effect of Annular Shape on Leaflet Curvature in Reducing Mitral Leaflet Stress. Circulation. 2002; 106:711-717.

38. Jimenez $\mathrm{JH}$, Yoganathan AP, et al. Effects of a saddle shaped annulus on mitral valve function and chordal force distribution: An in vitro Study. Annals of Biomedical Engineering. Vol. 31, pp. 1171-1181, 2003.

39. Adams DH, Anyanwu A. Pitfalls and limitations in measuring and interpreting the outcomes of mitral valve repair. J Thorac Cardiovasc Surg. 2006; 131(3):523-9.

40. Menicanti L, Di Donato $M$, Frigiola $A$, et al. Ischemic mitral regurgitation: intraventricular papillary muscle imbrication without mitral ring during left ventricular restoration. J Thorac Cardiovasc Surg 2005; 129: 440-2.

41. Kron IL, Green GR, Cope JT. Surgical relocation of the posterior papillary muscle in chronic ischemic mitral regurgitation. Ann Thorac Surg 2002:74:600-1.

42. Konertz W, Rombeck B, Hotz H.Short-term safety of the Acorn cardiac support device in patients with advanced heart failure. J Am Coll Cardiol 2000;35:182A.

43. Fukamachi K, McCarthy PM.Initial safety and feasibility clinical trial of the myosplint device. J Card Surg. 2005 Nov-Dec;20(6):S43-7

44. Gillinov AM, Wierup PN, Blackstone EH et al. Is repair preferable to replacement for ischemic mitral regurgitation?J Thorac Cardiovasc Surg. 2001;122(6):1125-41. 


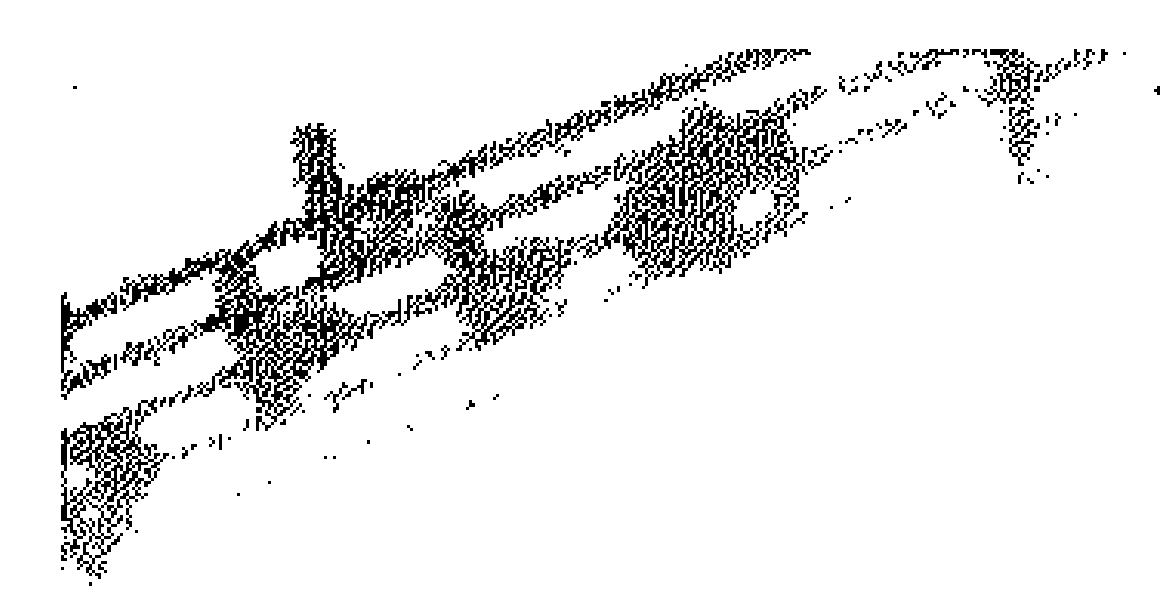

\author{
Insights on Left Ventricular and Valvular \\ Mechanisms of Recurrent Ischemic Mitral \\ Regurgitation After Restrictive Annuloplasty \\ and Coronary artery Bypass Grafting
}

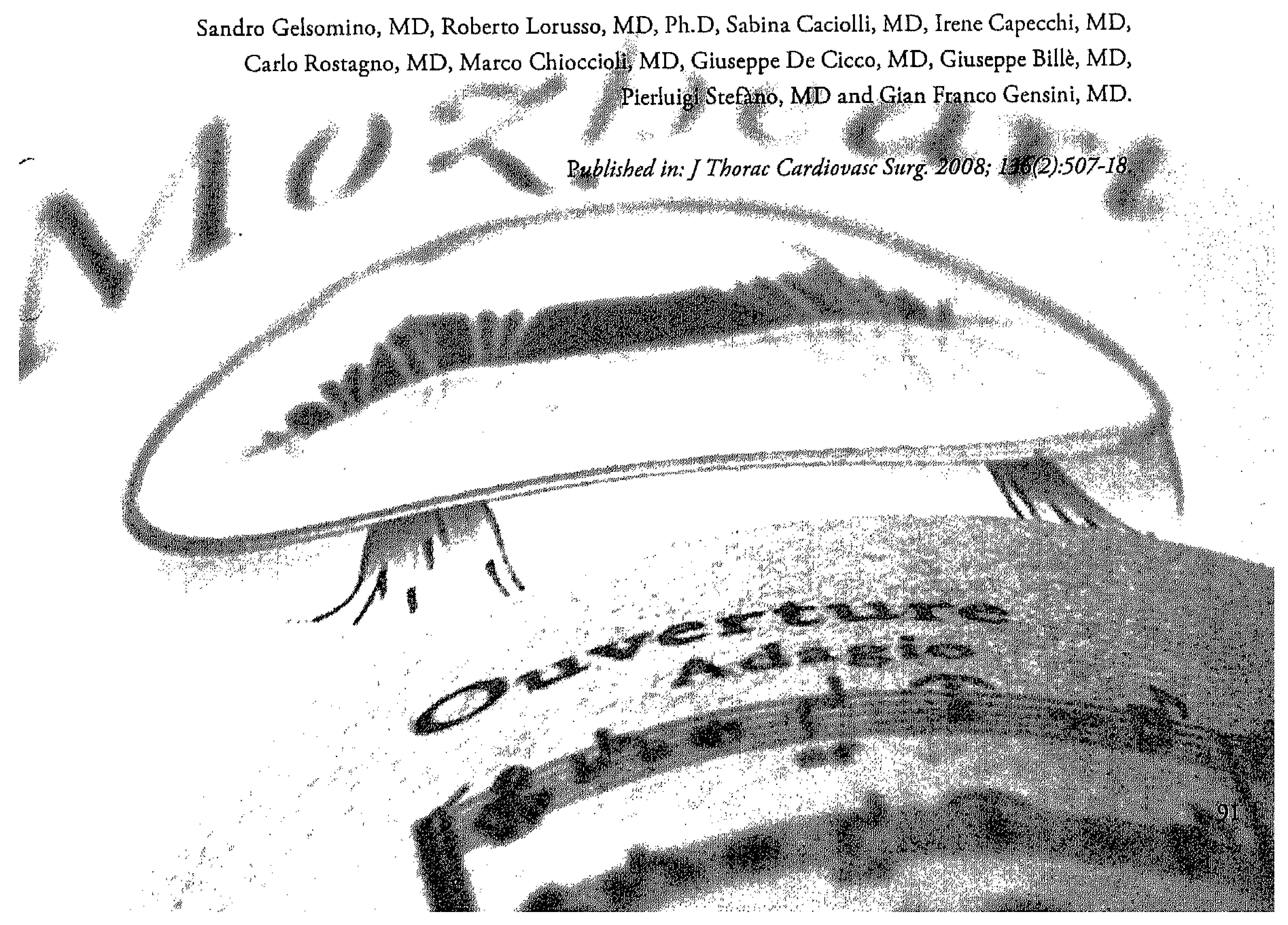


Background. We investigated leaflet and subvalvular configurations to identify mechanisms leading to recurrent mitral regurgitation after combined undersized mitral annuloplasty and coronary artery bypass and to preoperatively recognize patients who are unlikely to benefit from this approach.

Methods. Among 261 Subjects with chronic ischemic mitral regurgitation undergoing undersized annuloplasty and coronary bypass surgery in our Institution (Cardiac Surgery, Careggi Hospital, Florence, Italy) between September 2001 and September 2007, thirty-one were excluded: 4 had intraoperative annuloplasty failure, 12 showed residual regurgitation and 15 had incomplete echoes available. The study population consisted of 230 patients who were divided in two Groups: patients without (Group 1, n=176) or with (Group 2, n=54) late recurrent mitral regurgitation. Fifty normal healthy subjects were controls. Serial echoes were performed preoperatively, at discharge and at follow up appointments (early, median 6 months [Interquartile range 5-6]; late, median 33 months [17-51]).

Results. Subjects with late regurgitation had preoperatively a more symmetric tethering $(\mathrm{p}<0.001)$, a more accentuated anterior mitral leaflet tethering $(p<0.001)$ and a more restricted anterior leaflet excursion ( $p=0.003$ ) than Group 1. Postoperatively, tethering of posterior leaflet increased $(p<0.001)$ and it was predominant in both groups whereas the tethering of the anterior leaflet reduced at discharge $(\mathrm{p}=0.01$ and $\mathrm{p}=0.03$, respectively) remaining constant afterwards. Multivariable analysis showed anterior tethering angle $\geq 39.5^{\circ}(p<0.001)$, an anteriot/posterior tethering angle ratio $20.76(p<0.001)$, an anterior leaflet excursion angle $\leq 35^{\circ}(p=0.001)$ and a coaptation height $\geq 11 \mathrm{~mm}(\mathrm{p}=0.04)$ to be predictors of recurrent mitral regurgitarion.

Conclusions. Preoperative symmetric tethering with anterior mitral leaflet predominance was strongly associated with recurrence of MR. Measures of leaflet tethering resulted fundamental findings to identify ischemic patients who can really benefit from restrictive annuloplasty. Further larger studies are necessary to confirm our results. 
Chronic Ischemic Mitral Regurgitation (CIMR) results from a variable combination of infarction-induced subvalvular remodeling with subsequent leaflet tethering and annulus dilatation/flattening ${ }^{1,2}$. An established therapeutic approach to relieve CIMR, in association with coronary artery bypass grafting (CABG), is an undersized mitral ring annuloplasty (UMRA) which, by reducing the septal-lateral dimensions and the valve area, brings both mitral leaflets into apposition ${ }^{3}$. Nonetheless, after encouraging initial results ${ }^{4}$, different studies have revealed a significant proportion of patients developing recurrent mitral regurgitation (MR) during follow-up ${ }^{5-7}$ which has been mainly related to continued left ventricular remodeling and persistent leaflet tethering, ${ }^{7,8}$. In our previous experience ${ }^{9}, 72 \%$ of patients undergoing combined CABG and UMRA showed, at five-year control, recurrent MR. In addition, left ventricular reverse remodeling was observed only in $44.2 \%$ of the study population with $10.3 \%$ of patients showing further left ventricular dilatation. In the present study we investigated leaflet and subvalvular configurations before and after surgical annuloplasty to identify mechanisms leading to recurrent MR. Furthermore we attempted to determine whether preoperative tethering patterns and subvalvular configurations can accurately predict unsuccessful ischemic valve repair.

\section{METHODS}

\section{Patients population}

Among 261 Subjects with CIMR undergoing combined CABG and UMRA in our Institution (Cardiac Surgery, Careggi Hospital, Florence, Italy) between September 2001 and September 2007, thirty-one were excluded: 4 had intraoperative annuloplasty failure, 12 showed residual MR ( $\geq 2+$ at discharge) and 15 had incomplete echoes available. Definition and inclusion criteria are as previously reported'. Therefore, the study population consisted of 230 patients who were divided in two Groups: patients without (Group 1, $n=176$ ) or with (Group 2, $n=54$ ) late recurrent $M R$ (insufficiency $\geq 2+$ at late appointment in patients with no/trivial $M R$ at discharge). Fifty normal healthy subjects with no history of cardiovascular disease and with normal Doppler echocardiographic examination and who had a gender distribution, age and average body surface area similar to study patients were controls. Patient profiles are summarized in Table 1. Following the World Medical Association guidelines concerning ethical principles for medical research involving human subjects ${ }^{10}$, the study was approved by Institutional Ethics Board. Furthermore, all patients gave their informed consent. 
Table 1. Patients Profile

\begin{tabular}{|c|c|c|c|c|}
\hline & $\begin{array}{l}\text { All } \\
n=230\end{array}$ & $\begin{array}{l}\text { Group1 (MR-) } \\
\mathrm{n}=176\end{array}$ & $\begin{array}{l}\text { Group1 (MR+) } \\
\mathrm{n}=54\end{array}$ & $\mathbf{p}$ \\
\hline Age (years) & $67 \pm 7$ & $68 \pm 8$ & $66 \pm 7$ & 0.87 \\
\hline Gender $M / F$ & $\begin{array}{l}142 / 88 \\
(63.1 / 36.9)\end{array}$ & $\begin{array}{l}106 / 70 \\
(60.2 / 39.8)\end{array}$ & $\begin{array}{l}36 / 18 \\
(66.6 / 33.4)\end{array}$ & 0.07 \\
\hline NYHA class & $3[3-4]$ & $3[3-4]$ & $3[3-5]$ & 0.66 \\
\hline $\mathrm{CCS}$ angina class & $2[1-3]$ & $2[1-3]$ & $2[1-3]$ & $>0.9$ \\
\hline \multicolumn{5}{|l|}{ Euroscore } \\
\hline Additive & $8.1[5-9]$ & $8.0[5-9]$ & $8.2[5-8]$ & 0.8 \\
\hline Logistic & $15[10-18]$ & $14.8[11-18]$ & $15.1[10-18]$ & \\
\hline Hypertension & $89(38.7)$ & $67(38.1)$ & $22(40.7)$ & 0.6 \\
\hline Diabetes & $66(28.6)$ & $50(28.4)$ & $16(29.6)$ & 0.81 \\
\hline COPD & $31(12.7)$ & $23(13.0)$ & $8(12.5)$ & 0.9 \\
\hline Chronic renal disease & $35(15.2)$ & $26(14.8)$ & $9(16.6)$ & 0.77 \\
\hline Cerebral vascular disease & $25(10.8)$ & $18(10.2)$ & $7(12.0)$ & 0.63 \\
\hline Peripheral vascular disease & $16(6.9)$ & $12(6.8)$ & $4(7.4)$ & 0.83 \\
\hline Familiar history & $114(49.5)$ & $85(48.3)$ & $29(53.7)$ & 0.57 \\
\hline \multicolumn{5}{|l|}{ Myocardial infarction } \\
\hline$>3$ months & $175(76.1)$ & $132(75.0)$ & $43(79.6)$ & \\
\hline$s 3$ months & $55(23.9)$ & $44(25.0)$ & $11(20.4)$ & \\
\hline Inferior/Posterior & $121(52.6)$ & $98(55.7)$ & $23(42.5)$ & 0.8 \\
\hline Anterior/Septal & $20(8.7)$ & $14(7.9)$ & $6(11.1)$ & \\
\hline Lateral & $22(9.6)$ & $15(8.6)$ & $7(12.9)$ & \\
\hline Combined & $67(29.1)$ & $49(27.8)$ & $18(33.5)$ & \\
\hline Coronary vessel disease & $2[2-3]$ & $2[2-3]$ & $2[2-3]$ & \\
\hline 1 & $5(2.2)$ & $3(1.8)$ & $2(3.8)$ & \\
\hline 2 & $103(44.7)$ & $77(43.7)$ & $26(48.1)$ & 0.66 \\
\hline$\geq 3$ & $122(53.1)$ & $96(54.5)$ & $26(48.1)$ & \\
\hline Left Main & $43(18.6)$ & $32(18.1)$ & $11(20.3)$ & \\
\hline \multicolumn{5}{|l|}{ Medications } \\
\hline $\begin{array}{l}\text { Angiotensin-converting enzyme } \\
\text { inhibitors }\end{array}$ & $186(80,8)$ & $141(80.1)$ & $45(83.3)$ & \\
\hline B-adrenergic blockers & $124(53.9)$ & $96(54.5)$ & $28(51,8)$ & \\
\hline Long-acting nitrates & $105(45.6)$ & $82(46.6)$ & $23(42.5)$ & 0.71 \\
\hline Diuretics & $195(84.7)$ & $150(85.2)$ & $45(83.3)$ & \\
\hline Digitalis & $49(21.3)$ & $38(21.5)$ & $11(20.3)$ & \\
\hline Calcium antagonists & $35(15.2)$ & $26(14.7)$ & $9(16.6)$ & \\
\hline Preoperative IABP & $14(6.1)$ & $11(6.2)$ & $3(5.5)$ & 0.6 \\
\hline
\end{tabular}




\begin{tabular}{|c|c|c|c|c|}
\hline & $\begin{array}{l}\text { All } \\
\mathrm{n}=230\end{array}$ & $\begin{array}{l}\text { Group1 (MR-) } \\
\mathrm{n}=176\end{array}$ & $\begin{array}{l}\text { Groupl (MR+) } \\
\mathrm{n}=54\end{array}$ & $\mathbf{p}$ \\
\hline \multicolumn{5}{|l|}{ Surgery } \\
\hline Antegrade Cardioplegia & $208(90.4)$ & $158(89.7)$ & $50(92.5)$ & 0.6 \\
\hline $\begin{array}{l}\text { Antegrade and } \\
\text { RetrogradeCardioplegia }\end{array}$ & $22(9.6)$ & $18(10.3)$ & $4(7.5)$ & 0.16 \\
\hline $\mathrm{CPB}$ time (min) & $108[95-120]$ & $105[94-118]$ & $110[100-126]$ & 0.09 \\
\hline CCL time (min) & $90[70-98]$ & $81[66-90]$ & $95[70-101]$ & 0.06 \\
\hline \multicolumn{5}{|l|}{ Surgical Approach } \\
\hline Left atriotomy & $192(83.4)$ & $146(82.9)$ & $46(85.2)$ & 0.8 \\
\hline Transseptal & $21(9.2)$ & $17(9.7)$ & $4(7 . .4)$ & 0.23 \\
\hline Transseptal extended to LA roof & $17(7.4)$ & $13(7.4)$ & $4(7.4)$ & $>0.9$ \\
\hline \multicolumn{5}{|l|}{ Mitral Ring } \\
\hline${ }^{*}$ Carpentier Classic & $123(53.4)$ & $93(52.8)$ & $30(55.5)$ & 0.7 \\
\hline *Physio & $107(46.6)$ & $83(47.2)$ & $24(44.5)$ & 0.51 \\
\hline \multicolumn{5}{|l|}{ Ring size $(\mathrm{mm})$} \\
\hline $24 \mathrm{~mm}$ & $28[26-30]$ & $28[26-30]$ & $28[26-30]$ & $>0.9$ \\
\hline $26 \mathrm{~mm}$ & $12(5.2)$ & $9(5.1)$ & $3(5.5)$ & \\
\hline $28 \mathrm{~mm}$ & $87(37.8)$ & $68(38.6)$ & $19(35.3)$ & \\
\hline $30 \mathrm{~mm}$ & $88(38.3)$ & $67(38.1)$ & $21(38.8)$ & \\
\hline $\mathrm{CABG}$ & $43(18.7)$ & $32(18.2)$ & $11(20.4)$ & \\
\hline Anastomoses/patient & $2[2-3]$ & $2[2-3]$ & $2[2-3]$ & $>0.9$ \\
\hline Arterial graft/patient & $1[1-2]$ & $1[1-2]$ & $1[1-2]$ & $>0.9$ \\
\hline
\end{tabular}

\section{SURGERY}

All patients underwent associated CABG. For the purpose of this study, complete revascularization was accomplished when, at least one graft was placed distal to an approximately $50 \%$ diameter narrowing in each of the three major vascular system in which arterial narrowing of this severity was noted in a vessel $\geq 1.5 \mathrm{~mm}$ of diameter. It was not considered necessary to bypass all obstructed diagonal branches 
of the anterior descending or marginal branches of the circumflex coronary arteries for a classification of complete revascularization. Following this definition $100 \%$ patients underwent complete revascularization. The ring size was determined by standard measurements of the inter-trigonal distance and anterior leaflet height. A downsizing by two ring sizes was performed in all patients. After cardiopulmonary bypass (CPB), a transesophageal echocardiography (TEE) was performed to assess residual MR.

\section{ECHOCARDIOGRAPHY}

Serial trans-thoracic echoes (TTE) were performed preoperatively, at discharge and at follow up appointments (follow-up100\% complete). Median early follow up was 6 months (Interquartile range [IQR] 5-6), median late follow up was 33 months (IQR 17-51).

Echo examinations were carried out by two experienced echocardiographers (S.C. and M.C.) and stored on a magneto-optical disc for off-line analysis. Measurements were made off line by to cardiologists (I.C. and C.R.) blinded to severity of $M R$ and to the time when the images were made. Measurements and calculations were carried out as previously reported'. The reliability of echocardiographic measurements was assessed by calculating inter-observer and intra-observer interval of agreements of main direct measures used in this study in a different group of 20 subjects (10 MR, Appendix 1) ${ }^{11}$.

\section{Quantification of MR}

The following quantitative measurements were simultaneously employed to grade the severity of MR and final results were averages of measured values ${ }^{12-13}$ : (1) Quantitative Doppler: mitral and aortic stroke volumes were calculated and regurgitant volume (RV) was the difference between these 2 stroke volumes. The effective regurgitant orifice (ERO) area was the ratio of $R V$ to regurgitant time velocity integral (RTVI). (2) Proximal Isovelocity Surface Area (PISA) analyzed the proximal flow convergence and ERO was the ratio of regurgitant flow to regurgitant velocity. $\mathrm{RV}$ was the product of ERO by RTVI. For each measurement, minimum of three cardiac cycles were averaged. The severity of MR was graded on a scale of 1 to 4 according to American Society of Echocardiography Guidelines ${ }^{12}$. In patients with no/trivial MR by color Doppler, RV, and RF were used as calculated, and ERO was assumed as null. Mitral annular areas $\left(\mathrm{MA}_{s}\right)$ were obtained from mitral annular dimensions in apical long-axis, 4-chamber, and 2-chamber views, using an ellipsoid assumption ${ }^{14}$ and indexed by body surface area. 

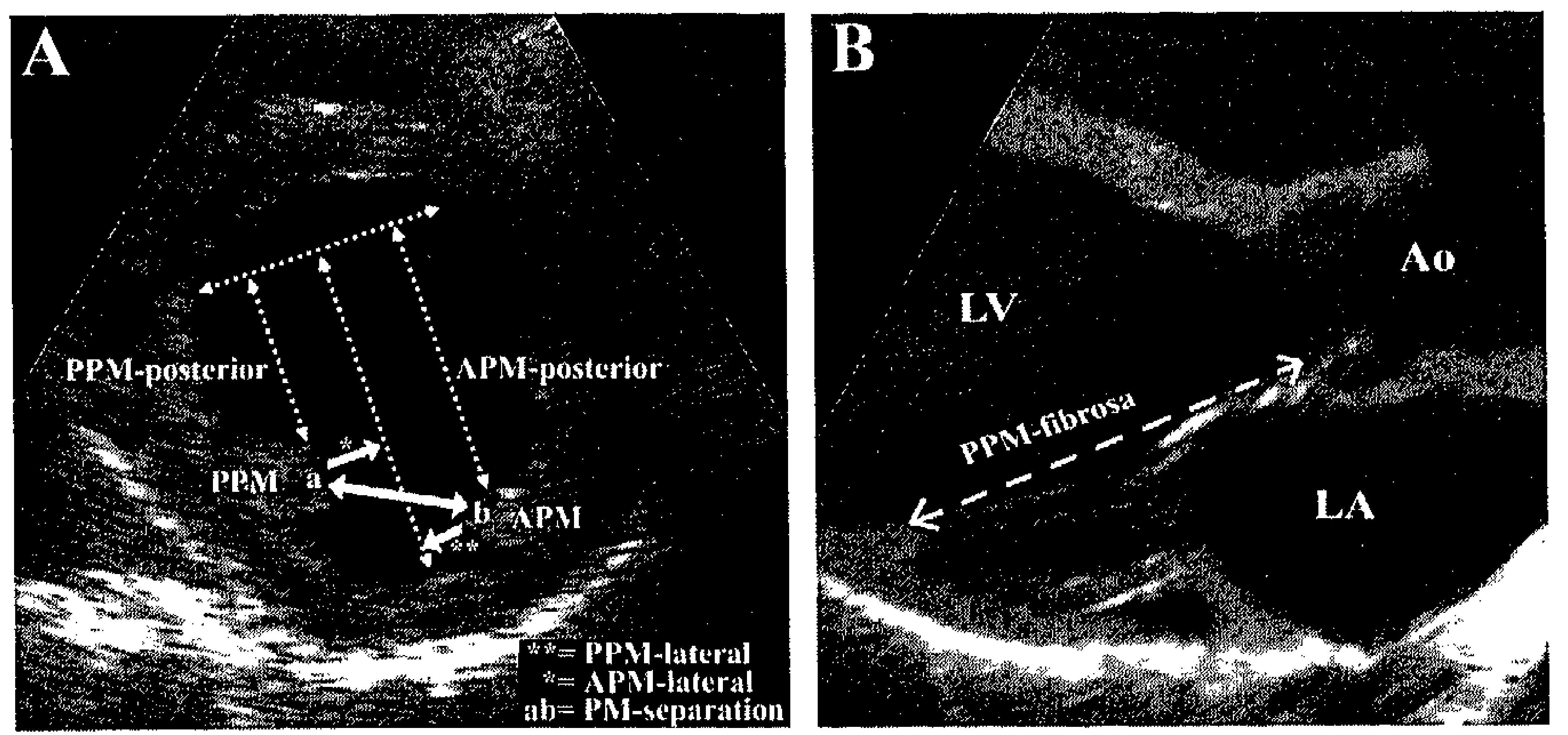

Figure 1. A) Papillary muscles displacement measured in the parasternal short axis view. B)PPM -fibrosa measured in long-axis view (see text).
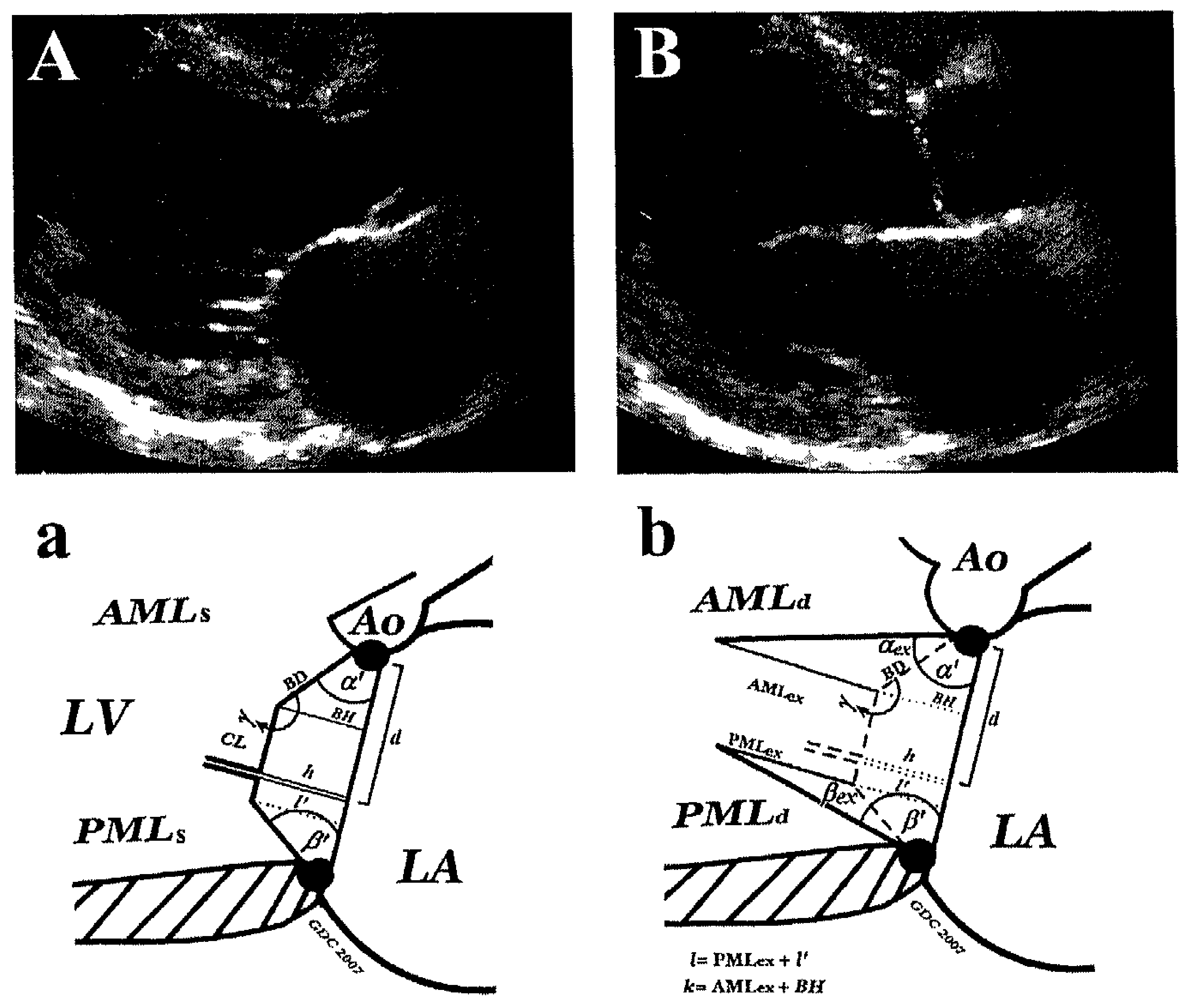

Figure 2. A, a. Method to quantify mitral leafler tethering in the parasternal long-axis view in midsystole. $\mathrm{CL}=$ Coaptation length; $\mathrm{d}=$ coaptation distance; $\mathrm{h}=$ coaptation height; $\alpha^{\prime}=$ Anterior mitral leaflet tethering angle; $\gamma=$ Bending angle; $\beta^{\prime}=$ posterior mitral leaflet tethering angle; B, b.Diastolic parasternal long-axis view. $\alpha \mathrm{EX}=$ Anterior mitral leaflet excursion angle; $\beta \mathrm{EX}=$ Posterior mitral leaflet excursion angle; AMLEX=Anterior mitral leaflet excursion; PMLEX=Posterior mitral leaflet excursion. 
The displacement of papillary muscles (PMs) was quantified as distances from well-defined anatomic landmarks at early and end systole ${ }^{15}$. The geometric chord defined by septal insertions and the mid-septal perpendicular line were used as references in the parasternal short-axis view (Figure 1A). Lateral and posterior displacements of anterior (APM) and posterior (PPM) papillary muscles were measured as distances from these fixed references. Separation between papillary muscles was directly measured. In the long axis view the apical displacement of the PPM was measured as the distance between the papillary muscle head and the fixed intervalvular fibrosis (annular-papillar distance, Figure 1B). The wall motion score indexes (WMSIs) of the basal and mid-posterior and inferior segments for the PPM and of basal and mid-lateral and anterior segments for the APM were also calculated ${ }^{15}$.

\section{Mitral leaflet tethering}

Mitral leaflets tethering in mid-systole was assessed in the parasternal long axis view (Figure 2). The mitral valve tenting area was calculated as the area enclosed between the annular line and the mitral valve leaflets. Posterior and apical displacement of the coaptation were measured as coaptation distance $d$ and coaptation height $h$, respectively whereas coaptation length (CL) was directly measured. The AML tethering angle, $\alpha$ ' the AML excursion angle $\alpha_{\mathrm{EX}}$ and distance $A M L_{\mathrm{Ex}}$, the PML tethering angle $\beta^{\prime}$, the PML excursion angle $\beta_{\mathrm{EX}}$ and distance PML $\mathrm{EX}_{\mathrm{EX}}$ and the bending angle $\gamma$ were obtained as explained in Appendix 2. Tethering patterns were defined according to Agricola and coworkers ${ }^{15}$. The anterior/posterior tethering angle ratio $\alpha^{\prime} / \beta^{\prime}$ was a quantitative measurements of tethering: the more this ratio was approaching 1 the more symmetric was the tethering.

\section{Statistical Analysis}

Variables were tested for normal distribution by the Kolmogorov-Smirnov test. Continuous data were expressed as mean \pm standard deviation, non-parametric data were presented as median and interquartile range and frequencies as proportions.

Data were compared by the Student's t-test, Mann Whitney U test, $\chi^{2}$ test and Fisher exact test where appropriate. Echocardiographic variables over time were analyzed by means of ANOVA repeated measures followed by Tukey post-hoc test. Kruskall Wallis and Friedman tests were used when variables were not normally distributed and the Dunn post-hoc test was employed for multiple comparisons.

Multivariable logistic regression analysis by means of a backward stepwise algorithm (cut-off for entry 0.05, for removal 0.10 ) was performed to select independent 
predictors of recurrent regurgitation and forty demographic, clinical, and echocardiographic parameters were investigated for their predictive value. To enhance the accuracy of the model, the number of variables was reduced using variable clustering ${ }^{16}$ until the number of variables to use as candidate in the regression analysis was $\leq \mathrm{m} / 10$, where for binary outcomes $\mathrm{m}$ is the number of patients in the less frequent outcome category ${ }^{17}$.

Model assumptions (linearity and additivity assumptions) were checked by piecewise cubic polynomials (spiline functions) and pooled interaction test ${ }^{18}$, respectively, and found to be satisfactory. Goodness of fit of the final logistic regression

Table 2. Preoperative Mitral Regurgitation

\begin{tabular}{lcllll}
\hline & $\begin{array}{l}\text { Controls } \\
\mathbf{n}=50\end{array}$ & $\begin{array}{l}\text { All } \\
\mathbf{n}=230\end{array}$ & $\begin{array}{l}\text { Group1 (MR-) } \\
\mathbf{n}=176\end{array}$ & $\begin{array}{l}\text { Group2 (MR+) } \\
\mathbf{n}=54\end{array}$ & $\mathbf{p}$ \\
\hline MR & & & & & \\
Grade & - & $3[3-4]$ & $3[3-4]$ & $3[3-4]$ & $>0.9$ \\
\hline
\end{tabular}

models was assessed with the Hosmer-Lemeshow statistic ${ }^{19}$ and predictive accuracy was assessed by the concordance index $(c)^{18}$. Internal validation of predictors generated by multivariable logistic regression was performed by means of bootstrapping techniques, with 1000 cycles and generation of OR and bias corrected $95 \%$ $\mathrm{CI}^{18}$. Optimal cutoff values were determined as the rounding cut-off that gives the maximum sum of sensitivity and specificity. This value should be the shoulder at the top left of the ROC (receiver operating characteristic curve). Bootstrapping techniques were employed to validate the results.

SPSS 12.0 (SPSS, Chicago, IL, USA) and Stats Direct 2.5.7 (Stats Direct, Sale, UK) were used for these calculations. Significance for hypothesis testing was set at the 0.05 two-tailed level.

\section{RESULTS}

Preoperative MR, PM Sisplacement leaflets tethering.

There was no significant difference between Groups regarding qualitative and quantitative MR data (Table 2). However, in the Group $1 \mathrm{MR}$ jet direction was posterior in the major part of patients, whereas it was central or anterior in most of patients in the Group 2. Furthermore, patients with recurrent mitral regurgitation had greater preoperative posterior and lateral displacement of the anterior papillary muscle (both, $p<0.001)$, a wider papillary muscle separation $(p=0.007)$ and a larger WMSI of the segments underlying the anterior papillary muscle $(\mathrm{p}=0.01)$. 

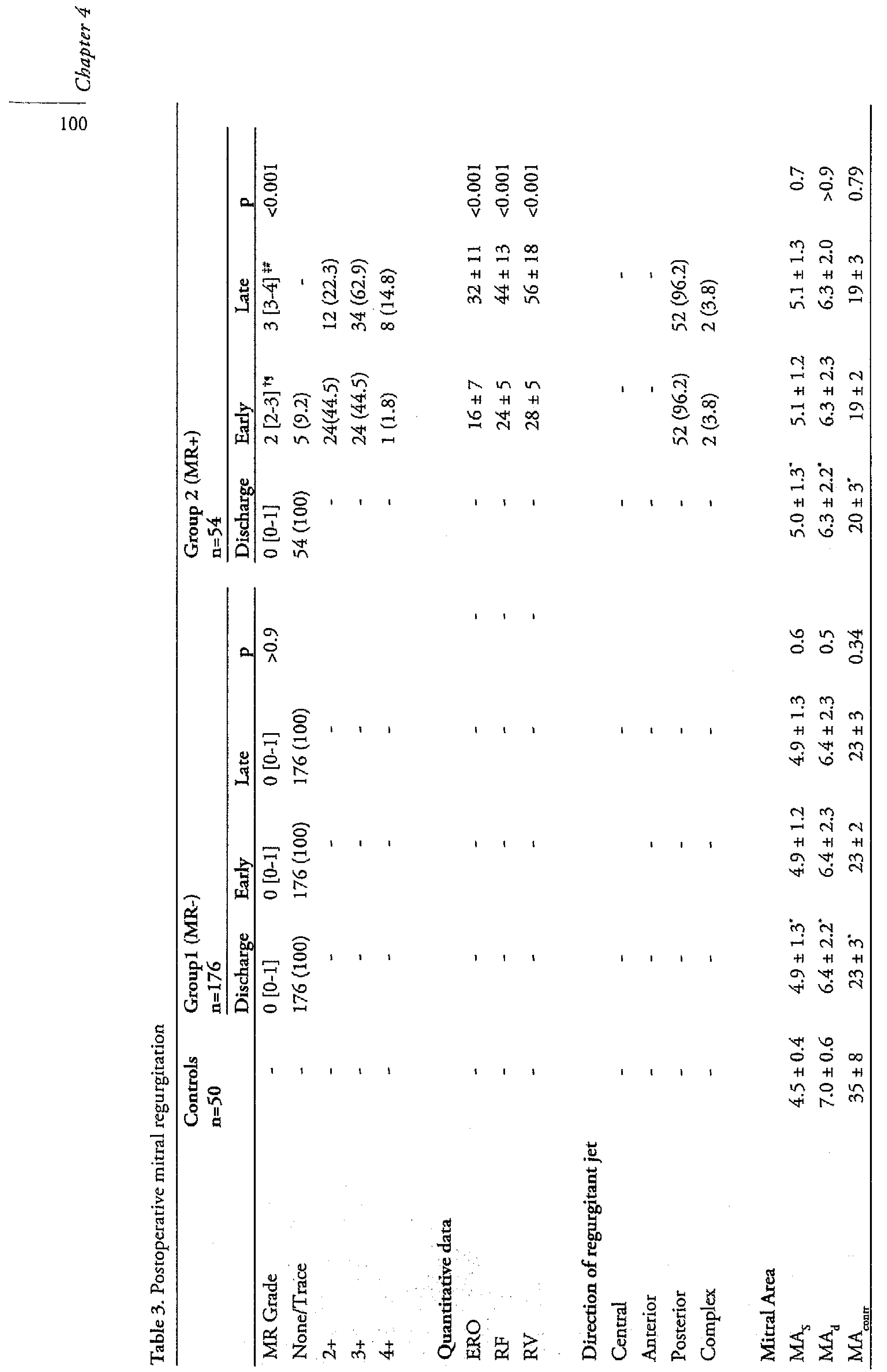


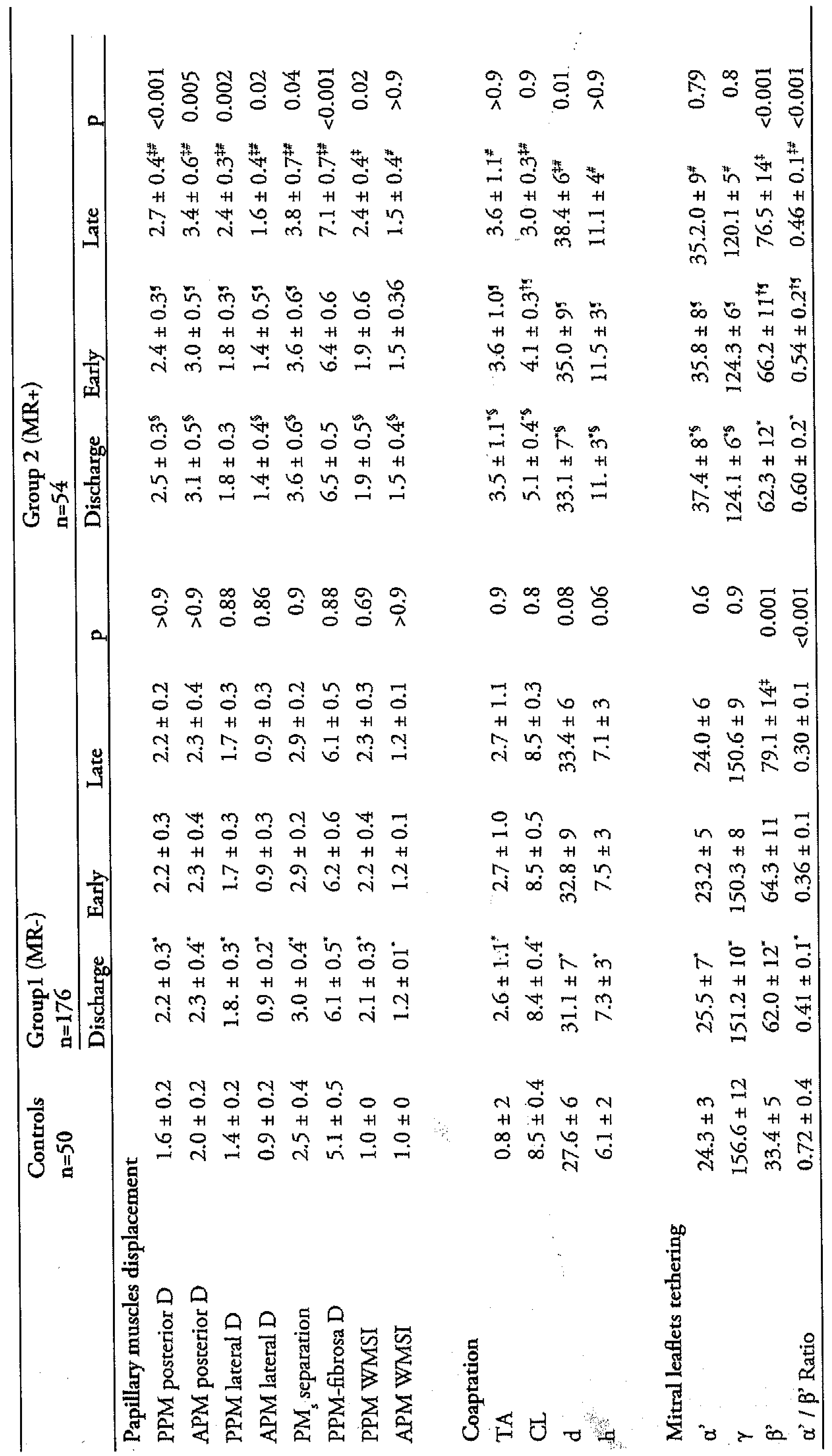



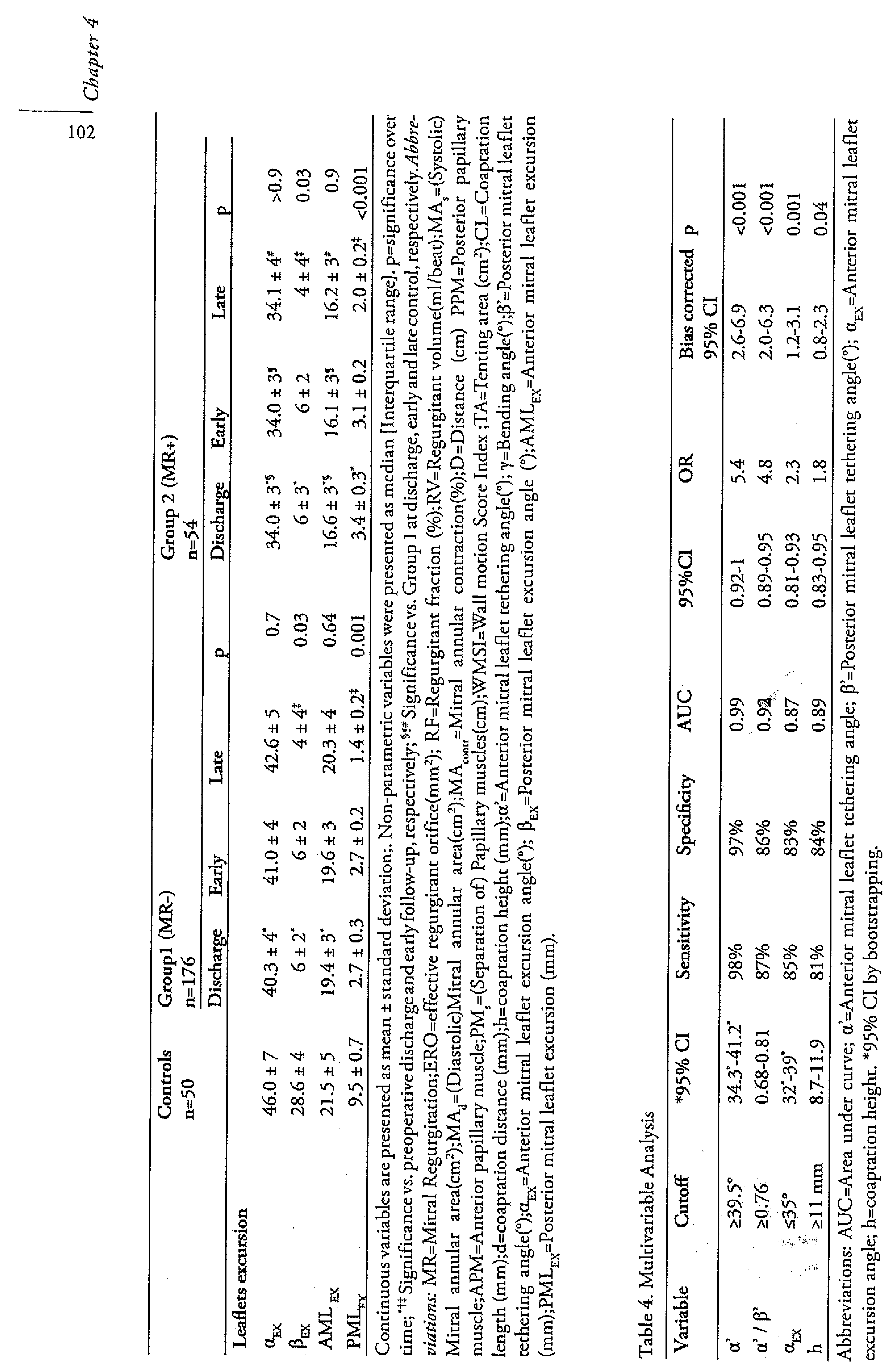
Contrastingly, WMSI of the segments underlying the posterior papillary muscle was higher in patients who did not show MR at postoperative control $(p=0.03)$, whereas posterior and lateral displacement of the posterior papillary muscle were comparable in two Groups ( $p>0.9$ and $p=0.7$, respectively). Moreover, tenting area $(\mathrm{p}<0.001)$ and coaptation height $(\mathrm{p}=0.004)$ were higher whereas coaptation length was lower $(p=0.006)$ in patients with recurrent MR. Contrastingly, the coaptation distance was comparable between Groups $(p=0.7)$. Additionally, both anterior tethering angle $\alpha^{\prime}(\mathrm{p}=0.001)$ and bending angle $\gamma(\mathrm{p}=0.01)$ were larger in Group 2, while the posterior tethering angle $\beta^{\prime}$ did not differ between groups $(p=0.66)$. When the degree of tethering was compared further in the two Groups, patients without postoperative MR had a more pronounced posterior leaflet tethering $(<0.001)$ with prevalent restriction of the posterior leaflet excursion $(\mathrm{p}<0.001)$. In contrast, patients who developed postoperative MR had a more accentuated anterior leaflet tethering $(\mathrm{p}<0.001)$, a more restricted excursion of the anterior leaflet $(\mathrm{p}<0.001)$ and a more symmetric tethering $(\mathrm{p}<0.001)$ when compared to Group 1.

\section{Postoperative MR, PMs displacement leaflets tethering.}

At postoperative control (Table 3) $96.2 \%$ of patients in the Group 2 showed a posterior MR whereas $3.8 \%$ had central or anterior regurgitation $(\mathrm{p}<0.001)$. Annular areas reduced from the preoperative value in both Groups $(\mathrm{p}<0.001)$ and remained constant over time. In patients with no recurrent $M R$, at discharge, indexes of posterior displacement (posterior papillary muscle, $\mathrm{p}=0.03$; anterior papillary muscle, $p=0.001$ ) and lateral displacement (posterior papillary muscle, $\mathrm{p}=0.02$; anterior papillary muscle, $\mathrm{p}=0.03)$ of both papillary muscles, $\mathrm{PMs}$ separation $(<0.001)$, annular-papillar distance $(\mathrm{p}=0.01)$ and wall motion score indices (posterior papillary muscle, $\mathrm{p}=0.02$, anterior papillary muscle, $\mathrm{p}=0.04$ ) were reduced significantly. All these parameters remained constant in this Group afterwards. In Group 2, all indices of displacement of both papillary muscles as well as WMSI at level of the posterior papillary muscle showed a not significant reduction at discharge, remained stable at early control, increasing significantly at late examination. In contrast, WMSI of the LV segments underlying the anterior papillary muscle did not show any significant change over time.

Furthermore, the coaptation distance $d$ reduced significantly in both Groups at discharge; however, whereas it kept constant in patients without recurrent $M R$, it significantly re-increased at late study in those with recurrent MR $(p=0.008)$. Differently, coaptation height significantly decreased at discharge $(\mathrm{p}<0.001$ and $\mathrm{p}=0.03$ in Group 1 and 2, respectively) and kept constant afterwards in both Groups. Coaptation length raised in the Group 1 at discharge $(p<0.001)$ remaining stable over time. In Group $2 \mathrm{CL}$ increased at discharge $(\mathrm{p}=0.02)$ but reduced at early $(\mathrm{p}=0.01)$ and late $(\mathrm{p}<0.001)$ controls. However coaptation length was at any time higher in 
Group 1 ( $\mathrm{p}<0.001$ ) which showed $100 \%$ patients with $\mathrm{CL} \geq 8 \mathrm{~mm}$ at postoperative controls. At discharge, the anterior leaflet tethering angle $\alpha$ ' significantly decreased $(p=0.01$ and $p=0.03$ in Groups 1 and 2, respectively), whereas the excursion angle $\alpha_{E X}(p=0.02$ and $p<0.001$ in Group 1 and 2, respectively) and the excursion distance $\mathrm{AML}_{\mathrm{EX}}$ of the anterior leaflet increased (both, $\mathrm{p}=0.02$ ). All these indices remained constant afterwards. In contrast, the posterior leaflet tethering angle $\beta^{\prime}$ raised at discharge (both, $\mathrm{p}<0.001$ ) whereas the excursion angle $\beta_{E X}$ (both, $\mathrm{p}<0.001$ ) and excursion distance $\mathrm{PML}_{\mathrm{EX}}$ (both, $\mathrm{p}<0.001$ ) reduced significantly in both Groups. These indices remained stable at early control, but, at late echocardiography, the tethering angle $\beta^{\prime}$ re-increased (both, $\mathrm{p}<0.001$ ) whereas the excursion angle $\beta_{\mathrm{EX}}$ (both 0.03 ) and the excursion distance $\mathrm{PML}_{\mathrm{EX}}$ (both $\mathrm{p}<0.001$ ) further reduced without difference between Groups.

The bending angle $\gamma$ significantly raised at discharge in patients without postoperative MR ( $\mathrm{p}=0.03)$ and kept constant at subsequent controls. Differently, the $\gamma$ showed a significant decrease in patients with postoperative $M R(p=0.008)$ remaining constant afterwards. When the degree of tethering was examined further in the two Groups, it became progressively more asymmetric in both Groups (Group 1, $\mathrm{p}<0.001$ vs. preoperative, $\mathrm{p}=0.03$ vs. discharge, $\mathrm{p}=0.03$ vs. early; Group $2, \mathrm{p}<0.001$ vs. preoperative, $\mathrm{p}=0.03$ vs. discharge, $\mathrm{p}=0.02$ vs. early).

\section{Valvular and Subvalyular determinants of Recurrent MR}

Multivariable regression analysis (Table 4) identified an anterior tethering angle $\alpha^{\prime} \geq 39.5^{\circ}(\mathrm{p}<0.001)$, an anterior/posterior tethering angle ratio $\alpha^{\prime} / \beta^{\prime} \geq 0.76$ $(\mathrm{p}<0.001)$, an anterior leaflet excursion angle $\alpha_{\mathrm{EX}} \leq 35^{\circ}(\mathrm{p}=0.001)$ and a coaptation height $h \geq 11 \mathrm{~mm}(\mathrm{p}=0.04)$ as independent predictors of recurrent MR. Internal validation of such multivariable analysis by means of bootstrapping, including the same variables of standard logistic regression model, further confirmed the significant predictive of early death (bootstrap $\mathrm{OR}=1.7,95 \%$ bias-corrected $\mathrm{CI}=0.6-3.8$ ). Finally, logistic regression model was reliable (Hosmer-Lemeshow test, $\mathrm{p}=0.6$ ) and accurate (c-index $=0.8)$.

\section{DISCUSSION.}

The main purpose of the present study was to investigate leaflets configurations in patients with CIMR before and after combined reductive annuloplasty and CABG and to identify characteristics of valvular as well as sub-valvular configurations responsible for recurrent ischemic regurgitation. Clinical data as well further treatment options offered for patients with discovery of residual/recurrent MR have been previously reported ${ }^{9}$ and have not been included in the present study. 
Previous studies ${ }^{7,20}$ demonstrated that the local remodeling of the LV segments supporting the posterior papillary muscle is a necessary condition for the development of MR. Accordingly, we found that remodeling of the regions supporting the posterior papillary muscle occurred in both Group as demonstrated by WMSI ${ }_{S}$ of these regions which were higher than normal in all patients. Nonetheless, in our experience, patients with recurrent MR had preoperatively a greater local LV remodeling of the LV segments underlying the anterior papillary muscle, a greater lateral and posterior displacement of the anterior papillary muscle and a wider $\mathrm{PM}_{\mathrm{S}}$ separation.

Interestingly, this different distribution of local wall abnormalities did not reflect, in our experience a different infarct localization confirming that mechanisms underlying CIMR are complex and not completely understood yet. However, our results support previous clinical findings reported by Agricola et a ${ }^{15}$ who described two Groups of patients with CIMR: likewise Group 2 in our study, their symmetric Group had prevalent posterior and lateral displacement of anterior papillary muscle (both $\mathrm{p}<0.04), \mathrm{PM}_{\mathrm{s}}$ separation and WMSI of the regions supporting the anterior papillary muscle (both $<0.001$ ).

After surgery, we showed that continued LV local remodeling occurs in both Groups and it predominantly involves the region of the LV supporting the posterior papillary muscle whereas the wall motion score index of segments underlying the anterior papillary muscle remained constant over time postoperatively in the entire patients cohort.

\section{Valvular Mechanism of Recurrent MR}

This study has demonstrated different baseline leaflet configuration in patient with or without recurrent mitral regurgitation. Patients without postoperative MR had a more asymmetric tethering pattern with a more pronounced posterior mitral leaflet tethering and with a prevalent restriction of the posterior leaflet excursion. Contrastingly, patients who developed postoperative MR had a more symmetric tethering pattern, a more accentuated anterior leaflet tethering, a more restricted anterior leaflet excursion and a smaller bending angle $\gamma$. The different tethering pattern translated into different preoperative MR jet directions: predominantly posterior in Group 1, mainly central in Group 2. Postoperatively, the tethering of the posterior leaflet increased and it was predominant in both groups at any stage postoperatively. In contrast, the tethering of the anterior leaflet reduced and AML excursion angle increased significantly early after surgery, temaining constant afterwards in both Groups. This is consistent with that reported by Zhu et a ${ }^{20}$ who observed a prevalent posterior leaflet tethering either in patients with $(n=25)$ 
or without $(n=6)$ postoperative $M R(p<0.05)$. Tethering of the posterior leaflet may be exacerbated by annuloplasty which, reducing annular size, can potentially shift the posterior annulus farther anteriorly, increasing its displacement relative to the papillary muscles without displacing the anterior annuls fixed at the aortic root ${ }^{21}$. However patients in Group 2 showed a mechanism of recurrent MR different from that of MR at baseline, with a tethering mechanism becoming progressively more asymmetric. In contrast with Kuwahara et $\mathrm{al}^{8}$ and Magne and coworkers ${ }^{22}$, in our experience, tethering of the posterior leaflet was not significant as a predictor of MR recurrence. However, these authors studied, recurrent/persistent and persistent MR, respectively, whereas, in our study residual MR was excluded. Persistent/residual MR is presumably due to immediate failure of the technique employed rather than LV remodeling ${ }^{23}$ and it is not surprising that regurgitation persists at late follow up in these patients. Contrastingly, we found an anterior tethering angle $\alpha^{\prime} \geq 39.5^{\circ}$ $(\mathrm{p}<0.001)$, an anterior/posterior tethering angle ratio $\alpha^{3} / \beta^{\prime} \geq 0.76(\mathrm{p}<0.001)$, an anterior leaflet excursion angle $\alpha_{\mathrm{EX}} \leq 35^{\circ}(\mathrm{p}=0.001)$ and a coaptation height $h \geq 11 \mathrm{~mm}$ $(\mathrm{p}=0.04)$ to be independent predictors of recurrent MR. Therefore, in accordance with Calafiore et al24, a coaptation distance (in our study coaptation height) $\geq 11$ $\mathrm{mm}$ was associated with a high risk of MR recurrence after UMRA. Nevertheless, as far as we know, this is the first demonstration that patients with predominant tethering of the anterior mitral leaflet had a higher likelihood to develop postoperative recurrent $M R$ after reductive annuloplasty. We can postulate that patients with more accentuated AML tethering cannot guarantee a sufficiently long anterior leaflet to cover the whole antero-posterior diameter and this is worsened by the augmented postoperative tethering of the posterior leaflet which further displaces the coaptation point posteriorly. Green et al. ${ }^{21}$ have reported, in an ovine model, that annuloplasty produces a markedly impaired motion of a "frozen" posterior mitral leaflet, transforming the mitral valve in unicuspid valve where the mechanism closure is performed only by the anterior leaflet and Kuwahara et $a l^{8}$ concluded that an anterior leaflet longer than antero-posterior annular diameter is required in the presence of restricted posterior leaflet to ensure successful repair. However, in our opinion, a long anterior leaflet could not always be sufficient to balance tethering forces secondary to postoperative remodeling and the coaptation length gained at the end of procedure could play a significant role in preventing MR recurrence. Indeed, patients in Group 1 had larger coaptation length (CL, $\mathrm{p}<0.001$ ) and 100\% showed a $\mathrm{CL} \geq 8 \mathrm{~mm}$ at postoperative controls. Interestingly, a subgroup of patients in the Group 2 showed postoperative MR even with a less tethered anterior mitral leaflet: in one of them the CL was small $(2.6 \mathrm{~mm})$ whereas in two the apposition surface was absent and the AML closed against the annulus. Thus, on the basis of the actual experience, we believe that, in all subjects especially those who are expected not to reverse remodel, a minimum CL of $8 \mathrm{~mm}$ should be achieved, as suggested by other authors ${ }^{25}$. Nonetheless, in patient with strongly tethered AML 
or with less tethered anterior leaflet but not sufficiently long to ensure a postoperative $\mathrm{CL} \geq 8 \mathrm{~mm}$, mitral regurgitation cannot be presumably eliminated by ring annuloplasty. In these patients a technique directly addressing $\mathrm{LV}$ remodeling or a chordal sparing mitral valve replacement should be considered. Additionally, a key point for achieving an appropriate leaflet coaptation and to ensure durable results is a true undersizing and, in this setting, a pivotal role can be played by the type of ring chosen. In the present experience we used only two rings (Carpentier's rigid or Physio semi-flexible rings). These two rings are not at all identical, thus, even while we have undersized by two sizes both rings, we might have been less restrictive with the Physio ring than with the Classic ring. However, in our previous experience", neither ring type nor ring size was predictive of recurrent MR.

Finally, patients with a more accentuated anterior mitral leaflet tethering and restrictive excursion could benefit from anterior basal chord cutting as a method of improving leaflet mobility and decreasing leaflet tethering. Nonetheless we have no experience with this technique and this issue deserves further investigation.

\section{LIMITATIONS OF THE STUDY}

Our study should be viewed in light of some inherent limitations.(1) Evaluation of mechanisms of recurrent ischemic mitral regurgitation were based on geometric assumptions and 2D echocardiographic measurements which are subject to imageplane positioning errors.

Hence it might be not accurate in shape distorted post-infarction left ventricles. The era of 3-D echocardiography has started potentially leading to new insights into LV remodeling, mitral apparatus shape and function ${ }^{26}$. Nonetheless the 3-D method is still on its way and is far for being validated, standardized and widely applied in the clinical practice ${ }^{27}$.(2) Viability testing was not performed in these patients. Therefore recurrence of MR might be also due to irreversible ischemic myocardial damage (not-viable myocardium). Furthermore, in relation to the papillary muscle displacement data presented, the revascularization of viable regions related to papillary muscle function might be the most important predictor of success with this operation. This limitation is shared with most of published papers. However, this issue deserves further investigation. (3)Postoperative evaluation of the coronary status was not assessed. It would have been helpful to differentiate between surgical failure (valve repair and CABG) and the progress of the coronary disease. (4)The issue of annulus re-shaping during annuloplasty has not been addressed. We employed only rigid/semi-flexible plane annuloplasty rings which may flatten the natural saddle shape causing greater tension on chordae and leaflets. Innovative annular rings have been recently introduced in the market which mimic the shape of healthy mitral annulus ${ }^{1,28-30}$. (5) Estimated cutoffs are known to be very susceptible to changes in the study population. We employed bootstrapping techniques to validate the results, 
nonetheless, it has also been documented that the sensitivity/specificity associated with these cutoffs are overly optimistic.

\section{STRENGTH OF THE STUDY}

The study does not display most of common clinical and methodological pitfalls that limit results from many studies of mitral valve repair ${ }^{23}$. The patient cohort was large and the echocardiographic follow-up was detailed and 100\% complete. In addition, our study population was more homogeneous than in other reports: all patients underwent associated CABG, they had no concomitant MV procedures and the entire cohort was uniform regarding the MV ischemic leaflet dysfunction. Moreover, only true "recurrent" MR was studied, excluding those patients with "residual" MR in whom the insufficiency was presumably never eliminated at surgery. Additionally, we undertook valve sizing in a standardized fashion and the degree of undersizing was homogeneous over the 5-year-period of the study. Finally, we employed only two rings (rigid and semi-flexible) which were equally distributed in the two groups.

\section{CONCLUSIONS}

Preoperative symmetric tethering with AML predominance was strongly associated with recurrence of MR. Measures of leaflet tethering, which are not systematically employed in the clinical practice, resulted, in our experience, fundamental findings to identify ischemic patients who can really benefit from restrictive annuloplasty. Further larger studies are necessary to confirm our findings.

\section{REFERENCES}

1. Gorman JH 3rd, Jackson BM, Enomoto Y, Gorman RC. The effect of regional ischemia on mitral valve annular saddle shape.Ann Thorac Surg 2004;77:544-8.

2. Otsuji $\mathrm{Y}$, Handschumacher MD, Schwammenthal E, et al. Insights from three-dimensional echocardiography into the mechanism of functional mitral regurgitation: direct in vivo demonstration of altered leaflet tethering geometry. Circulation 1997;96:1999 -2008.

3. Bolling SF, Pagani FD, Deeb GM, Bach DS. Intermediate term outcome of mitral reconstruction in cardiomyopathy. J Thorac Cardiovasc Surg 1998;115(2):381-6.

4. Bolling SF, Deeb GM, Bach DS. Mitral valve reconstruction in elderly, ischemic patients. Chest 1996;109(1):35- 40

5. McGee EC, Gillinov AM, Blackstone EH et al. Recurrent mitral regurgitation after annuloplasty for functional ischemic mitral regurgitation. J Thorac Cardiovasc Surg 2004, 128(6):916-24. 
6. Serri K, Bouchard D, Demers $P$ et al. Is a good perioperative echocardiographic result predictive of durability in ischemic mitral valve repair? J Thorac Cardiovasc Surg 2006;131:523-529.

7. Hung J, Papakostas L, Tahta SA et al. Mechanism of recurrent ischemic mitral regurgitation after annuloplasty: continued LV remodeling as a moving target. Circulation 2004;110 (Suppl. II):II85-II90.

8. Kuwahara E, Otsuji Y, Iguro $Y$ et al. Mechanism of recurrent/persistent ischemic/functional mitral regurgitation in the chronic phase after surgical annuloplasty: importance of augmented posterior leaflet tethering. Circulation 2006;114(Suppl.):1529-1534.

9. Gelsomino S, Lorusso R, De Cicco G et al. Five-year echocardiographic results of combined undersized mitral ring annuloplasty and coronary artery bypass grafting for chronic ischaemic mitral regurgitation. Eur Heart J. 2008 Jan;29(2):231-40.

10. World Medical Association Declaration of Helsinki: Ethical principles for medical research involving human subjects.http://www.wma.net/e/policy/b3.htm (Accessed January 9, 2008).

11. Bland MJ, Altman DJ. Statistical method for assessing agreement between two methods of clinical measurements.Lancet 1986;1:307-310.

12. Zoghbi WA, Enriquez-Sarano M, Foster $E$ et al. Recommendations for evaluation of the severity of native valvular regurgitation with two-dimensional and Doppler echocardiography. J Am Soc Echocardiogr 2003;16: 777-802.

13. Grigioni F, Enriquez-Sarano M, Zehr KJ, Bailey KL, Tajik AJ. Ischemic Mitral Regurgitation. Long-term outcome and prognostic implications with quantitative Doppler assessment. Circulation. 2001;103(13):1759-64.

14. Vijayaraghavan G, Boltwood CM, Tei C, Wong M, Shah PM. Simplified echocardiographic measurement of the mitral annulus. Am Heart J 1986;112(5): 985-91.

15. Agricola E, Oppizzi M, Maisano $F$ et al. Echocardiographic classification of chronic mitral regurgitation caused by restricted motion according to tethering pattern. Eur J Echocardiography 2004; 5: 326-34.

16. Romesburg HC. Cluster analysis for researchers. North Carolina: Lulu Press; 2004.1-330.

17. Harrel FE., Lee KL, Califf RM, Pryor DB, Rosati RA. Regression modelling strategies for improved prognostic prediction. Statistics in Medicine 1984; 3: 143-52.

18. Harrel FE Jr, Lee KL, Mark DB. Multivariable Prognostic Models: Issues in developing models, evaluating assumprions and adequacy, and measuring and reducing errors. Stats Med 1996:36187.

19. Hosmer DW, Lemeshow S. Applied Logistic regression. $2^{\text {nd }}$ ed.New York: Wiley-Interscience; 2000:143-202.

20. Zhu F, Otsuji $Y$, Yotsumoto $G$ et al. Mechanism of persistent ischemic mitral regurgitation after annuloplasty: importance of augmented posterior mitral leaflet tethering. Circulation. 2005;112(Suppl):I396-401.

21. Green GR, Dagum P, Glasson JR et al. Restricted posterior leaflet motion after mitral ring annuloplasty. Ann Thorac Surg. 1999;68(6):2100-6. 
22. Magne J, Pibarot P, Dagenais F, Hachicha Z, Dumesnil JG, Senechal M. Preoperative posterior leaflet angle accurately predicts outcome after restrictive mitral valve annuloplasty for ischemic mitral regurgitation.Circulation. 2007;115(6):782-91.

23. Adams DH, Anyanwu AC. Pitfalls and limitations in measuring and interpreting the outcomes of mitral valve repair. J. Thorac Cardivasc Surg 2006; 131:523-9.

24. Calafiore AM, Di Mauro M, Gallina S et al. Mitral valve surgery for chronic ischemic mitral regurgitation.Ann Thorac Surg. 2004;77(6):1989-97.

25. Braun J, Bax JJ, Versteegh MI et al. Preoperative left ventricular dimensions predict reverse remodeling following restrictive mitral annuloplasty in ischemic mitral regurgitation. Eur J Cardiothorac Surg. 2005;27(5):847-53.

26. Tsukiji M, Watanabe N, Yamaura Y et al. Three-Dimensional Quantitation of Mitral Valve Coaptation by a Novel Software System with Transthoracic Real-Time Three-Dimensional Echocardiography.J Am Soc Echocardiogr. 2007; [Epub ahead of print].

27. Ryan I.P, Jackson BM, Parish LM et al. Mitral valve tenting index for assessment of subvalvular remodeling.Ann Thorac Surg. 2007;84(4):1243-9.

28. Votta E, Maisano F, Bolling SF, Alfieri O, Montevecchi FM, Redaelli A. The Geoform diseasespecific annuloplasty system: a finite element study. Ann Thorac Surg. 2007;84(1):92-101.

29. Salgo IS, Gorman JH, Gorman RC, et al. Effect of Annular Shape on Leaflet Curvature in Reducing Mitral Leaflet Stress. Circulation. 2002; 106:711-717.

30. Jimenez JH, Liou SW, Padala $M$ et al. A saddle-shaped annulus reduces systolic strain on the central region of the mitral valve anterior leaflet. J Thorac Cardiovasc Surg. 2007;134(6):1562-8 
Bland -Altman limits of agreement for intra-observer and inter-observer variability

\begin{tabular}{lllll}
\hline Variable & & $\begin{array}{l}\text { Mean } \\
\text { difference }\end{array}$ & $\begin{array}{l}\text { Standard } \\
\text { deviation }\end{array}$ & $\begin{array}{l}\text { 95\% limits } \\
\text { of agreement }\end{array}$ \\
\hline ESV(ml) & Intra-observer (IC) & 1.3 & 1.6 & $-1.8-4.4$ \\
& Intra-observer (CR) & 0.5 & 2.7 & $-4.8-5.8$ \\
& Inter observer & 1.6 & 2.9 & $-4.0-7.3$ \\
LVEF (\%) & Intra-observer (IC) & 2.4 & 1.8 & $-1.1-6.0$ \\
& Intra-observer (CR) & 2.5 & 2 & $-1.5-6.5$ \\
& Inter observer & 2.3 & 2.8 & $-3.0-7.8$ \\
ERO $\left(\mathrm{mm}^{2}\right)$ & Intra-observer (IC) & 0.4 & 2.1 & $-3.8-4.7$ \\
& Intra-observer (CR) & 0.5 & 1.8 & $-3.1-4.1$ \\
& Inter observer & 1.4 & 2.4 & $-3.4-6.2$ \\
h (mm) & Intra-observer (IC) & 0.1 & 0.3 & $-0.4-0.7$ \\
& Intra-observer (CR) & 0.1 & 0.2 & $-0.4-0.6$ \\
& Inter observer & 0.2 & 0.4 & $-0.5-1.0$ \\
$\alpha^{\prime}\left({ }^{\circ}\right)$ & Intra-observer (IC) & 1.4 & 1.9 & $-2.2-5.1$ \\
& Intra-observer (CR) & 1.7 & 1.8 & $-1.8-5.2$ \\
& Inter observer & 1.8 & 2.1 & $-2.3-6.0$ \\
$\beta^{\prime}\left({ }^{\circ}\right)$ & Intra-observer (IC) & 2.1 & 2.2 & $-2.2-6.4$ \\
& Intra-observer (CR) & 1.5 & 2.2 & $-2.8-5.9$ \\
& Inter observer & 1.4 & 3.5 & $-5.5-8.3$ \\
\hline
\end{tabular}

Intra-observer and inter-observer relative differences were $<5 \%$ for all parameters. The Bland-Altman method showed excellent agreement between intra-observer and inter-observer measurements in both low and high values of echocardiographic parameters. Observers: observer 1: Irene Capecchi, (IC); observer 2: Carlo Rostagno (CR).Inter-observer (observer 1-observer 2).

Abbreviations: $E S V=e n d$ systolic volume; $L V E F=l e f t$ ventricular ejection fraction; $E R O=$ effective regurgitant orifice (area); $h=$ coaptation height; $\alpha^{\prime}=$ Anterior mitral leaflet tethering angle, $\beta^{\prime}=$ Posterior mitral leaflet tethering angle. 


\section{Tethering Measurements.}

The tethering angle of the anterior mitral leaflet (AML) $\alpha^{\prime}$ was measured as follows:

$$
\alpha^{\prime}=\arcsin \left(\frac{B H}{B D}\right)
$$

where $\mathrm{BH}$ was the bending height between the annular line and the bending angle $\gamma$ created by tethering of intermediate or strut chordae in the body of the anterior leaflet, and $\mathrm{BD}$ was the bending distance between the bending point and the anterior annulus.

The AML excursion angle $\alpha_{\mathrm{EX}}$ were obtained as:

$$
\alpha_{\mathrm{ex}}=\left[\left(\arcsin \frac{K}{A M L d}\right)-\left(\arcsin \frac{B H}{B D}\right)\right]
$$

Where $A M L_{d}$ and $\mathrm{K}$ were the length of the anterior leaflet and the distance measured from the tip of the AML to the annular line, respectively, measured at the end of diastole.

Thus the anterior leaflet excursion $\mathrm{AML}_{\mathrm{EX}}$ was calculated as:

$$
\mathrm{AML}_{\mathrm{EX}}=\mathrm{AML}^{*} \sin \left[\left(\arcsin \frac{K}{A M L d}\right)-\left(\arcsin \frac{B H}{B D}\right)\right]
$$

Similarly, the posterior mitral leaflet (PML) tethering angle $\beta$ ' was calculated as follows:

$$
\beta^{\prime}=\arcsin \left(\frac{P M L s}{l^{\prime}}\right)
$$


Where PMLs was the mid-systolic PML and $l$ 'was the distance between the PML coaptation point and the annular line.

Angle of PML excursion $\left(\beta_{\mathrm{EX}}\right)$ and excursion distance $\left(\mathrm{PML}_{\mathrm{EX}}\right)$ were calculated as:

$$
\begin{aligned}
\beta_{\mathrm{EX}}= & {\left[\left(\arcsin \frac{l}{P M L d}\right)-\left(\arcsin \frac{P M L s}{l^{\prime}}\right)\right] ; \mathrm{PML}_{\mathrm{EX}}=\mathrm{PML}^{*} \sin } \\
& {\left[\left(\arcsin \frac{l}{P M L}\right)-\left(\arcsin \frac{P M L s}{l^{\prime}}\right)\right] }
\end{aligned}
$$

Where PMLd and 1 were length of the posterior leaflet and the distance measured from the tip of the PML to the annular line, respectively, measured at the end of diastole.

The $\alpha^{\prime} / \beta^{\prime}$ ratio was calculated to verify the tethering symmetry.

The bending angle $(\gamma)$ was calculated as:

$$
\gamma=90^{\circ}+\arccos \left(\frac{B H}{B D}\right)
$$




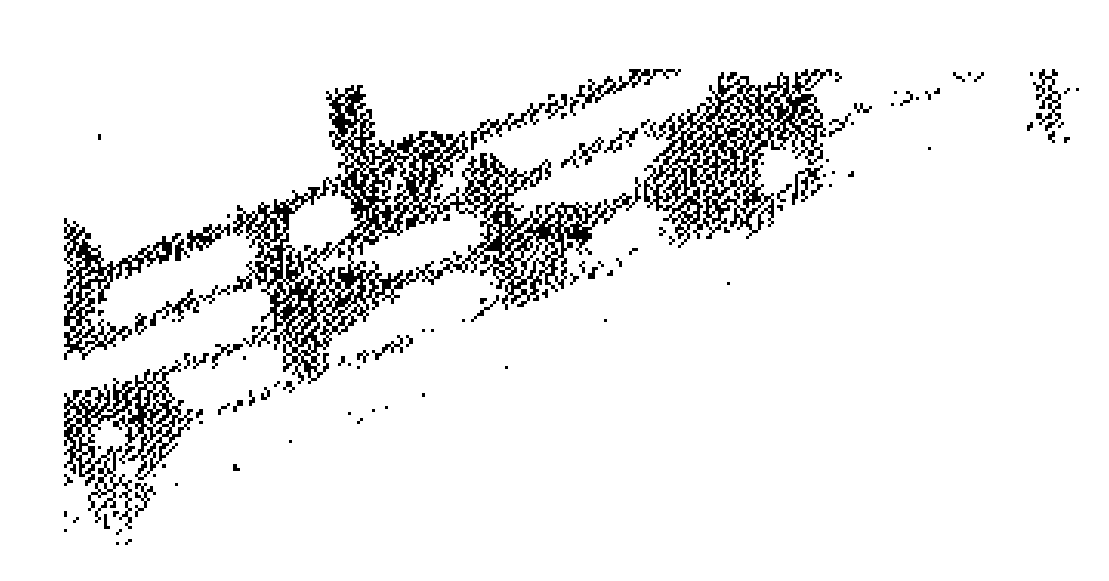

\section{Impact of Anterior Leaflet Tethering on Recurrence of Ischemic Mitral Regurgitation after Restrictive Annuloplasty}

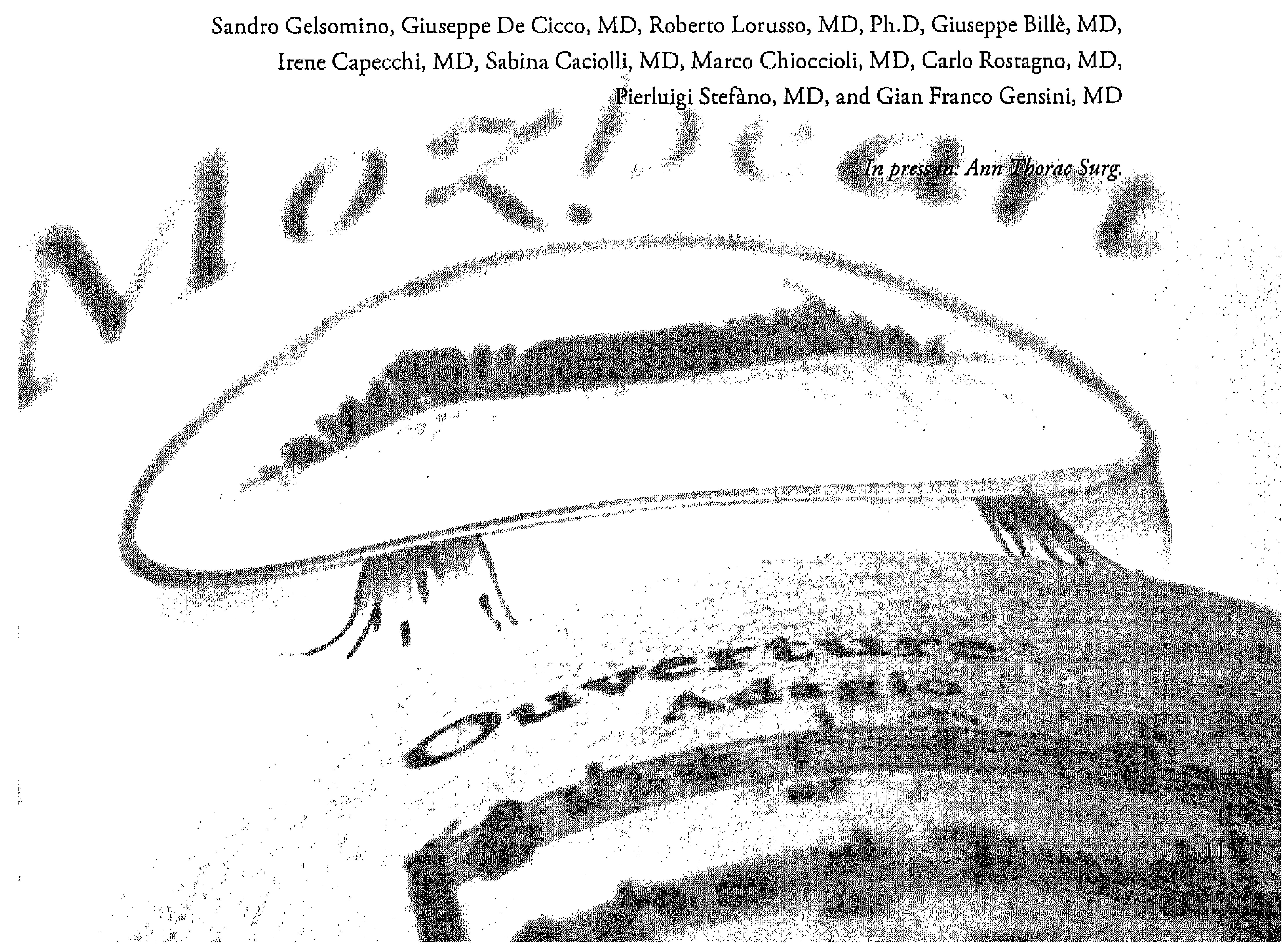




\section{ABSTRACT}

Background: We hypothesized that a specific leaflet configuration with anterior mitral leaflet (AML) tethering predominance is related to higher postoperative MR recurrence. Relationships between AML tethering and recurrent mitral regurgitation were investigated.

Methods: In 278 patients with surgical annuloplasty for chronic ischemic mitral regurgitation and 50 matched normal subjects, posterior and lateral displacement of papillary muscles (PMs), the $\mathrm{AML}$ and posterior mitral leaflet (PML) tethering angles and their ratio, coaptation length and the MR grade were quantified before and ar follow up (median, 38 months [Interquartile range 15-60 months]). Patients were divided in 5 Groups on the basis of the preoperative AML tethering angle $\alpha$ :Group 1, normal/slight AML tethering ( $\mathrm{n}=33$ ), Group 2, mild AML tethering ( $\mathrm{n}=55$ ), Group 3, moderate AML tethering $(n=63)$, Group 4, moderate-severe AML tethering $(n=64)$ and Group 5 , severe AML terhering $(n=63)$.

Results: Subjects wirh AML tethering $\geq$ moderate-severe had preoperatively a more symmetric tethering ( $\mathrm{p}<0.001)$, a more accentuated anterior papillary muscle displacement $(\mathrm{p}<0.001)$ and a larger anterior local remodeling $(\mathrm{p}=0.02)$. Recurrence of $M R$ was significantly higher in patients with moderate-severe/severe AML tethering $(p<0.001)$. Postoperatively, tethering of posterior leaflet increased $(p<0.001$ ) and it was predominant without difference berween groups, resulting in a more asymmetric tethering in most of patients with recurrent $M R(p<0.001)$.

At multivariable logistic regression the AML tethering angle $\alpha$ ' was the primary determinant of MR recurrence after adjustment of known risk factors $(p<0.001)$.

Conclusions: The grade of preoperative AML tethering was strongly associated with recurrence of MR. Assessment of leaflet tethering and PMs displacement by 2-D echocardiography should be incorporated into clinical risk assessment and prediction models. 
A standard therapeutic approach to relieve ischemic mitral regurgitation (IMR) is a ring annuloplasty which reduces mitral annular area by bringing the dilated posterior annulus anteriorly to shorten the anterior posterior dimension (or septallateral in anatomic terms) and bring the leaflet into apposition ${ }^{1.5}$. Although often effective initially ${ }^{6}$, long term failures are increasingly recognized ${ }^{7-10}$. Green et al. ${ }^{11}$ have reported in an ovine model that annuloplasty produces a markedly impaired motion of a "frozen" posterior mitral leaflet, transforming the mitral valve in unicuspid valve where the mechanism closure is performed only by the anterior mitral leaflet (AML) and Kuwahara et al ${ }^{12}$ demonstrated that an AML longer than antero-posterior annular diameter is required in the presence of restricted posterior leaflet to ensure successful repair. Thus, the extent of preoperative anterior mitral leaflet (AML) tethering could play a crucial role in determining a successful repair. Nonetheless, little is known regarding the real impact of the AML tethering on postoperative recurrence of MR.

In our recent experience we have shown that annuloplasty failure is related to wider preoperative AML tethering ${ }^{13}$ and this hypothesis has been tested for recurrent MR late after surgery. However, the altered leaflet geometry and the recurrent MR could represent both the result of abnormal left ventricular (LV) function and geometry rather than the change in valve anatomy being primary.

Therefore, in the present study we investigated the "true" impact of AML tethering on unsuccessful ischemic valve repair in relation to other factors influencing MR recurrence and we tested the hypothesis that a specific leaflet configuration with $A M L$ tethering predominance is related to higher postoperative MR recurrence.

\section{METHODS}

\section{Subjects}

Among 316 patients with chronic ischemic mitral regurgitation undergoing combined coronary artery bypass grafting (CABG) and undersized mitral ring annuloplasty (UMRA) in our Institution (Cardiac Surgery, Careggi Hospital, Florence, Italy) between September 2001 and January 2008, thirty-eight were excluded: 4 had intraoperative annuloplasty failure, 14 showed residual MR ( $\geq 2+$ at discharge) and 20 had incomplete echoes available. Therefore, the study population consisted of 278 patients. Fifty normal healthy subjects with no history of cardiovascular disease and with normal Doppler echocardiographic examination and who had a gender distribution, age and average body surface area similar to study patients were 
controls. Definition, inclusion and exclusion criteria were as previously reported ${ }^{7}$. Median follow-up was 38 months (IQR 15-60).

Following the World Medical Association guidelines concerning ethical principles for medical research involving human subjects, the study was approved by Institutional Ethics Board. Furthermore, all patients gave their informed consent.

\section{SURGERY}

All patients underwent associated CABG. For the purpose of this study, complete revascularization was accomplished when, at least one graft was placed distal to an approximately $50 \%$ diameter narrowing in each of the three major vascular system in which arterial narrowing of this severity was noted in a vessel $\geq 1.5 \mathrm{~mm}$ of diameter. It was not considered necessary to bypass all obstructed diagonal branches of the anterior descending or marginal branches of the circumflex coronary arteries for a classification of complete revascularization. Following this definition $100 \%$ patients underwent complete revascularization. The ring size was determined by standard measurements of the inter-trigonal distance and anterior leaflet height. A downsizing by two ring sizes was performed in all patients.

\section{Echocardiographic Measurements}

Echo examinations were carried out preoperatively and at follow up appointment by two experienced echocardiographers (S.C. and M.C.) and all measurements were made off line as previously reported ${ }^{7}$ by to cardiologists (I.C. and C.R.) blinded to severity of MR and to the time when the images were made. The reliability of echocardiographic measurements was assessed by calculating inter-observer and intra-observer interval of agreements in a different group of 20 subjects (10 MR, Appendix 1$)^{14}$.

\section{Papillary muscles displacement}

Lateral and posterior displacements of anterior (APM) and posterior (PPM) papillary muscles were measured as previously reported ${ }^{13}$. In the long axis view the apical displacement of the PPM was measured as the distance between the papillary muscle head and the fixed inter-valvular fibrosis (annular-papillar distance) ${ }^{15}$. The wall motion score indexes (WMSI) of the basal and mid-posterior and inferior segments for the PPM and of basal and mid-lateral and anterior segments for the APM were also calculated ${ }^{15}$. 
Mitral leaflet configuration was quantified in the parasternal long-axis view in mid- systole and early diastole (Figure 1). The angles $\alpha^{\prime}$ and $\beta$ ', the bending angle $\gamma$, the leaflets excursion distances (PML $\mathrm{EX}_{\mathrm{EX}}$ and $\mathrm{AML}_{\mathrm{EX}}$, respectively) and leaflets excursion angles ( $\alpha_{\mathrm{EX}}$ and $\beta_{\mathrm{EX}}$, respectively) were calculates as previously reported ${ }^{13}$. The anterior/posterior tethering angle ratio $\alpha^{\prime} / \beta^{\prime}$ was a quantitative measurements of tethering: the more this ratio was approaching 1 the more symmetric was the tethering. The distances $\mathrm{d}$ and $\mathrm{h}$ representing posterior and apical displacement of the coaptation and the coaptation length were directly measured.

\section{Patients Classification}

Patients were divided in quintiles with baseline $\alpha^{\prime}$ values of $<27.2^{\circ}$ (Normal/ slight AML tethering, Group 1, $n=33$ ), 27.2 $\leq 32.1^{\circ}$ (Mild AML tethering, Group 2, $n=55$ ), $32.2^{\circ} \leq 35.9^{\circ}$ (Moderate AML tethering, Group 3, $n=63$ ), 36. $0^{\circ} \leq 39.5$ (Moderate-severe AML tethering, Group 4, $n=64$ ) and $>39.5^{\circ}$ (Severe AML tethering, Group 5, $n=63$ ), respectively. Patient characteristics are summarized in Table 1. No difference was found in preoperative and operative variables between Groups.

\section{Statistical Analysis}

Variables were tested for normal distribution by the Kolmogorov-Smirnov test. Continuous data were expressed as mean \pm standard deviation, non-parametric data were presented as median and interquartile range (IQR) and frequencies as proportions.

Variable were compared across $\alpha^{\prime}$ categories with the ANOVA, Kruskall-Wallis and $\chi^{2}$ tests with Tukey and Dunn post-hoc tests, where appropriate.

Multivariable logistic regression analysis was performed to asses the effect of preoperative AML tethering on recurrence of MR. Forty demographic, clinical, and echocardiographic parameters were investigated for their predictive value. To enhance the accuracy of the model, the number of variables was reduced using variable clustering ${ }^{16,17}$. Model assumptions (linearity and additivity assumptions) were checked by piecewise cubic polynomials (spiline functions) and pooled interaction test $^{18}$, respectively, and found to be satisfied. Goodness of fit of the final logistic regression models was assessed with the Hosmer-Lemeshow statistic ${ }^{19}$ and predictive accuracy was assessed by the concordance index (c) ${ }^{18}$. For presentation purpose we first analyzed the main effect of preoperative AML tethering an then we investigated model wit interactions between $\alpha^{\prime}$ and other key risk factors selected on the basis of results of our previous experiences 7,20 , and from data of published papers ${ }^{12,21}$. A significance level of $\mathrm{p}<0.01$ was used as criterion for reporting interactions. Internal 

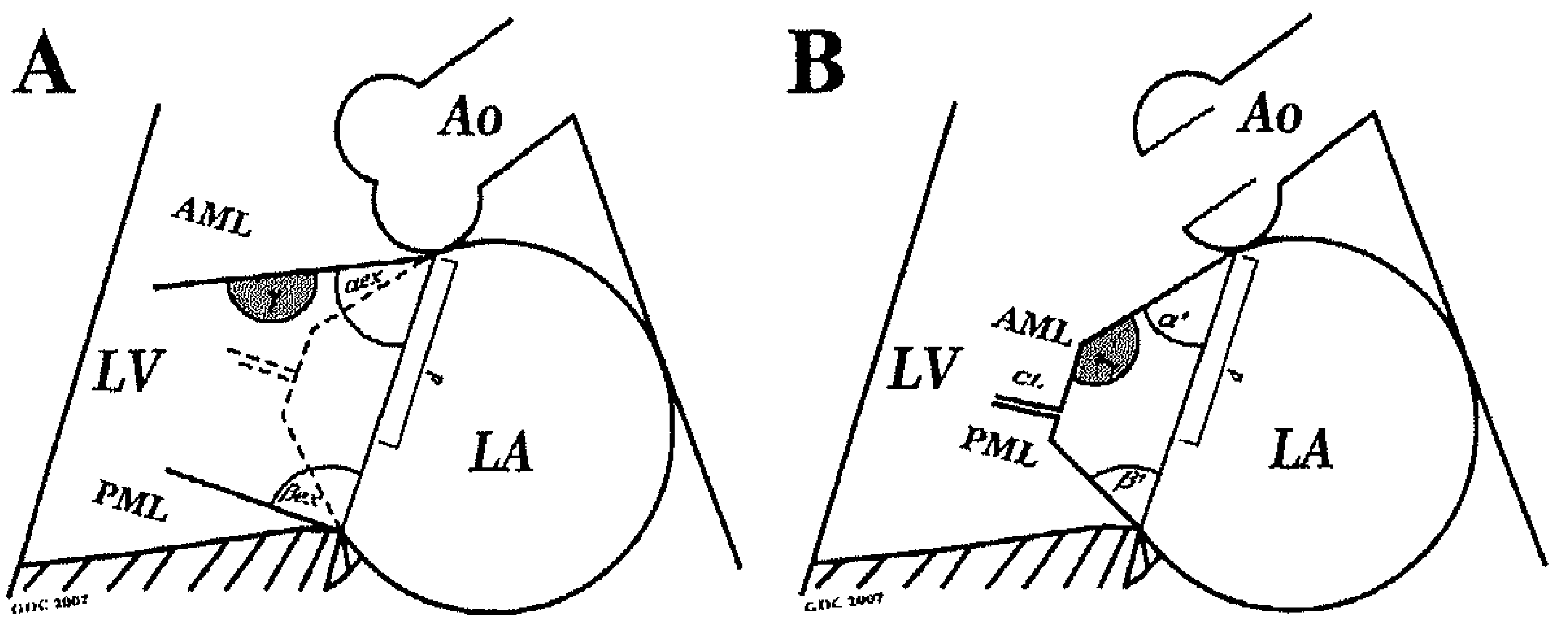

Figure 1. Methods to quantify the mitral leaflet configurations in the parasternal long axis 2-dimensional echocardiogram (see text) in diastole $(A)$ and systole $(B)$. The angles $\alpha$ ' and $\beta$ ' represent the grade of Anterior Mitral Leaflet (AML) and Posterior Mitral Leaflet (PML) tethering: The bending angle $\gamma$ is the angle created by tethering of intermediate or strut chordae in the body of the anterior leaflet. Anterior and Posterior leaflet excursion angles ( $\alpha_{\mathrm{EX}}$ and $\beta_{\mathrm{EX}}$, respectively) were calculated from systolic closure to diastolic maximal opening.

Abbreviations: $d$ : coaptation distance; CL: Coaptation length.

validation of predictors generated by multivariable logistic regression was performed by means of bootstrapping techniques, with 1000 cycles and generation of $O R$ and bias corrected $95 \% \mathrm{Cl}^{18}$.

SPSS 12.0 (SPSS, Chicago, IL, USA) and Stats Direct 2.5.7 (Stats Direct, Sale, UK) were used for these calculations.

\section{RESULTS}

\section{Mitral Regurgitation}

There was no significant difference between Groups regarding quantitative MR data in the preoperative period (Table 2). However, tenting area $(p<0.001)$ and coaptation height $(\mathrm{p}=0.03)$ were higher in patients with severe or moderate-severe AML tethering. In addition, in these Groups, preoperative MR jet direction was central in most of patients, whereas it was posterior in the major part of patients of the other Groups (Figure 2A).

At follow-up 62 patients (22.3\%) showed recurrent MR (insufficiency $\geq 2+$ in patients with no/trivial MR at discharge): it occurred in $47.6 \%$ patients with severe, $28.1 \%$ moderate-severe, $17.4 \%$ moderate and $10.9 \%$ mild preoperative AML tethering $(\mathrm{p}<0.001)$. None in the normal/slight AML tethering Group showed recurrent MR. At postoperative control $90.3 \%$ of patients with MR recurrence had posterior jet without difference between Groups (Figure 2B). 


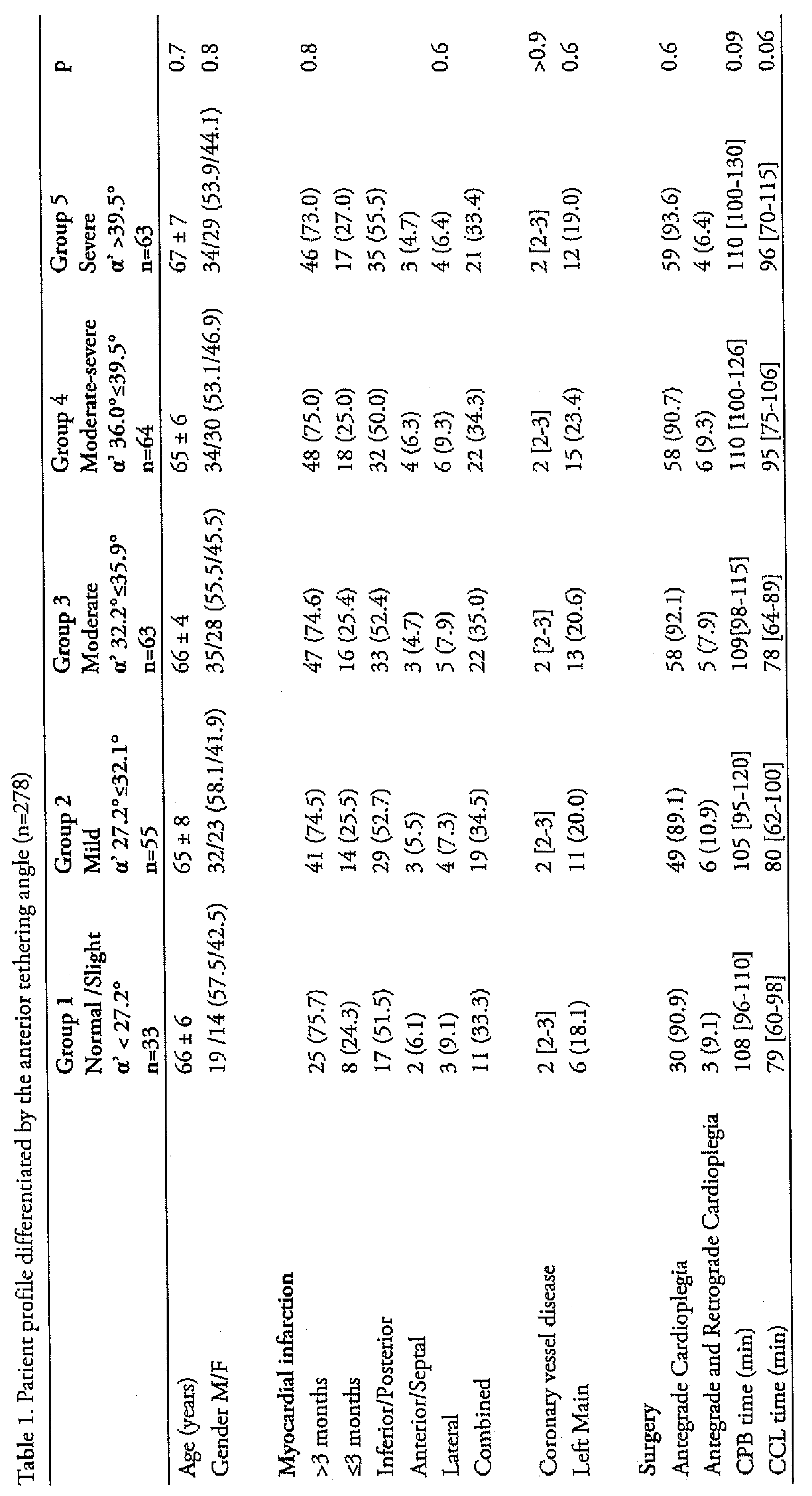




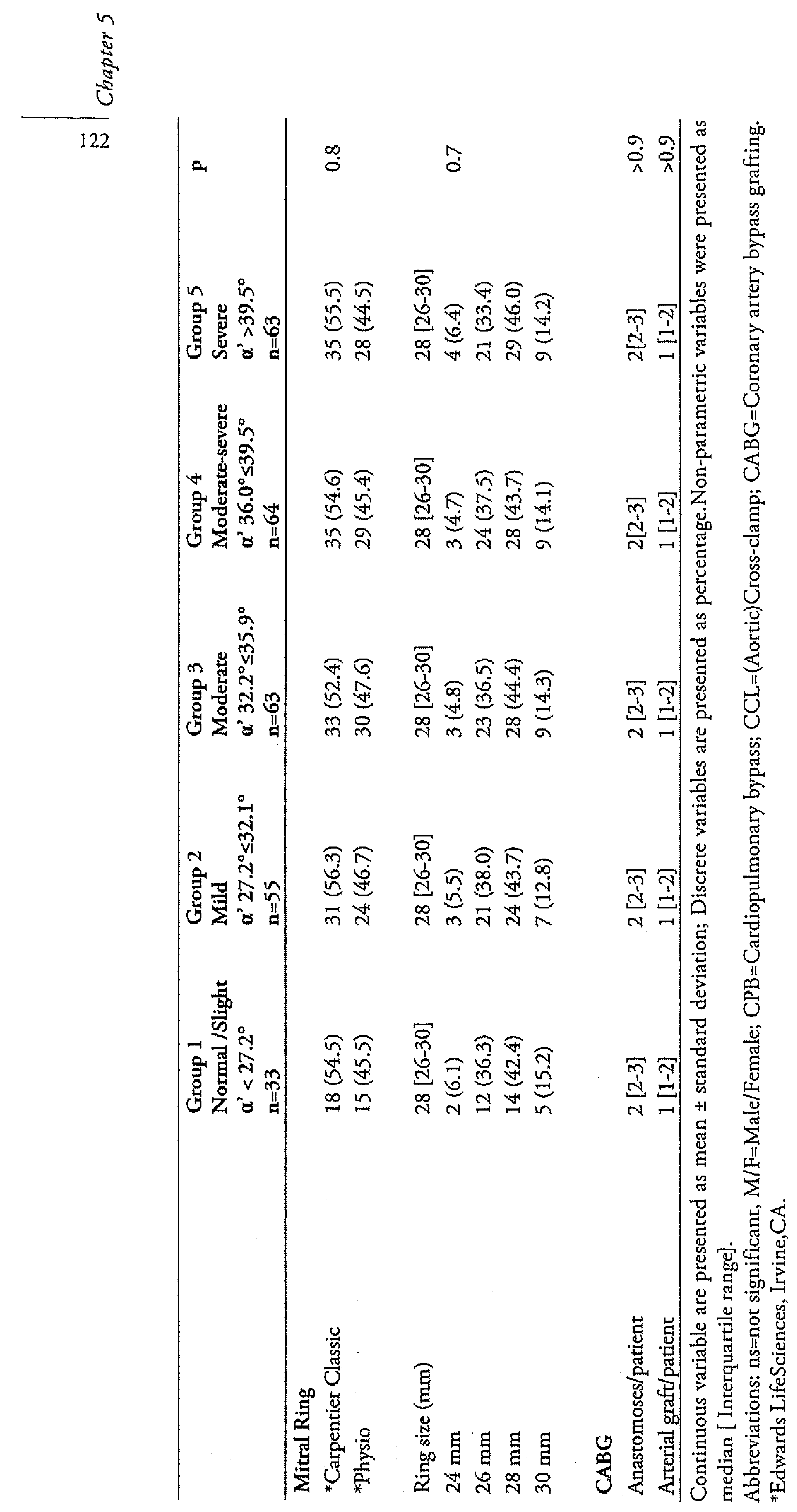


The tenting Area reduced from preoperatively in Groups 1-3 (Table 3, $\mathrm{p}<0.001$ ) whereas it increased significantly in Groups 4 and 5 ( $p<0.001$ ). Furthermore, coaptation length increased in patients with preoperative AML tethering $\leq$ moderate $(p<0.001)$ whereas in the remnants it reduced significantly $(p<0.001)$. However coaptation length was at any time lower in Groups 4-5 ( $\mathrm{p}<0.001$ ) which showed $100 \%$ patients with $\mathrm{CL} \leq 8 \mathrm{~mm}$ at follow-up.

Additionally, the coaptation distance $d$ reduced significantly in Groups 1-3 whereas it significantly re-increased at late study in the other Groups $(p=0.001)$. Finally, coaptation height significantly decreased in all Groups but remained higher in patients with preoperative moderate-severe /severe AML tethering.

\section{Papillary muscle Displacement}

As shown in Figure 3A, patients with moderate-severe/severe AML tethering had greater preoperative posterior and lateral displacement of the anterior papillary muscle (both, $\mathrm{p}<0.001)$, a wider papillary muscle separation $(\mathrm{p}=0.001)$ and a larger WMSI of the segments underlying the anterior papillary muscle $(\mathrm{p}=0.02)$. Contrastingly, WMSI of the segments underlying the posterior papillary muscle was higher in patients in Groups 1-3 ( $\mathrm{p}=0.03)$, whereas posterior and lateral displacement of the posterior papillary muscle as well as PPM fibrosis were comparable between Groups (Figure 3B).

At follow-up (Figure 3C), indices of posterior and lateral displacement of the anterior papillary muscle, PMs separation and WMSI of segments underlying the anterior papillary muscle, reduced significantly from the preoperative value only in Groups 1-3 ( $\mathrm{p}<0.001)$ whereas they remained stable in patients with moderate severe and severe AML tethering with values significantly higher compared to other Groups $(\mathrm{p}<0.001)$.

In contrast, indexes of a posterior and lateral displacement of PPM as well as WMSI at level of the PPM reduced significantly in patients Groups 1-3. Contrastingly, all these indexes increased in patients with $\geq$ moderate-severe AML tethering $(p<0.001$, Figure $3 \mathrm{D})$ with values significantly higher compared to other Groups $(\mathrm{p}<0.001)$.

\section{LEAFLET TETHERING}

In the preoperative period, the excursion angle of the AML and the bending angle $\gamma(p<0.01)$ were lower in Groups $4-5$ (Figure 4A, $p<0.01)$. Contrastingly, the PML tethering angle $(p=0.66)$ and the posterior leaflet excursion angle $(p=0.52)$ were not different between Groups (Figure 4B).

At follow up the excursion angle $\alpha_{\mathrm{EX}}$ of the anterior leaflet increased (Figure 4C, $\mathrm{p}<0.001$ ) in patients of Groups 4-5. In Groups 1-3 this increment was not significant 

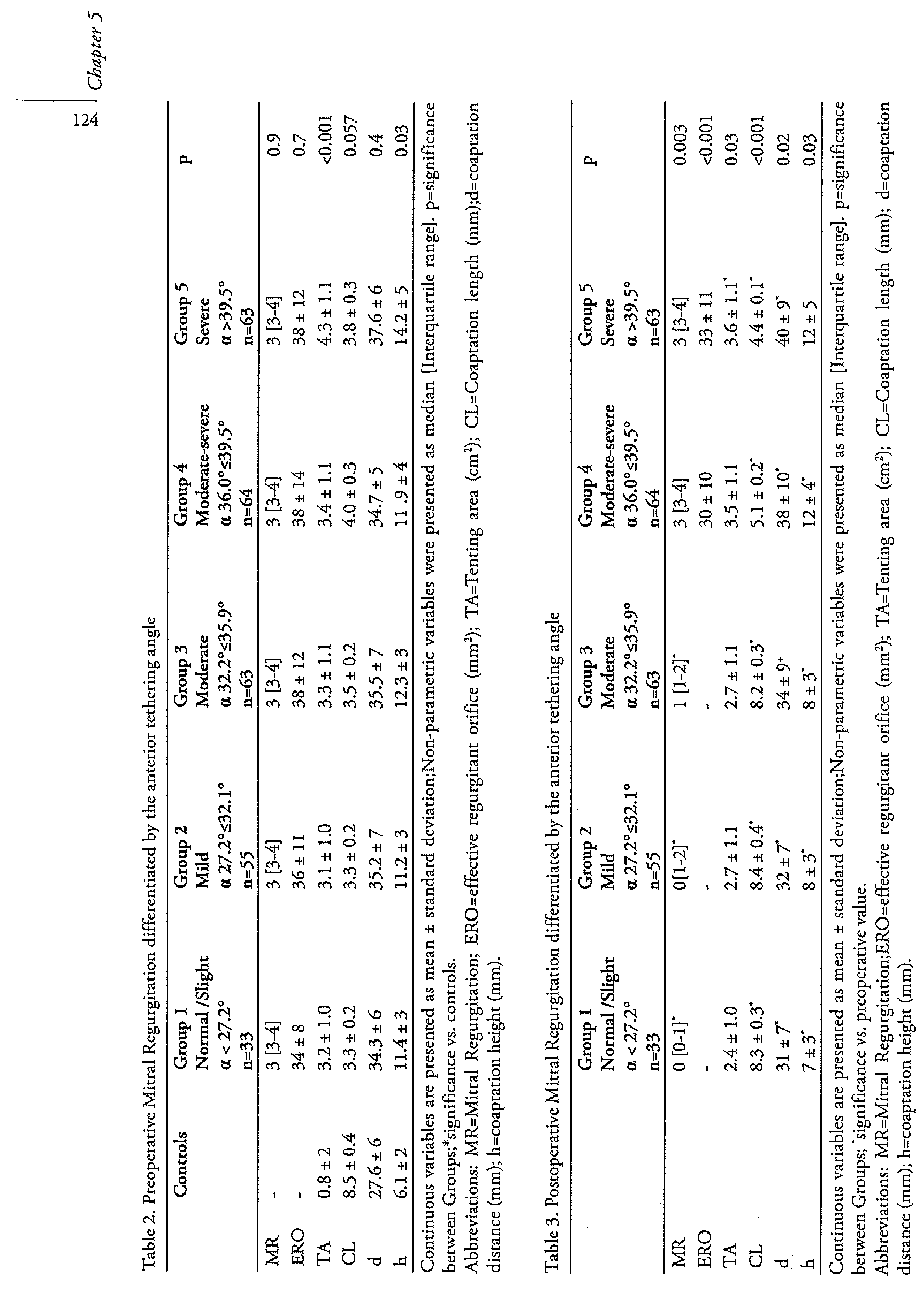


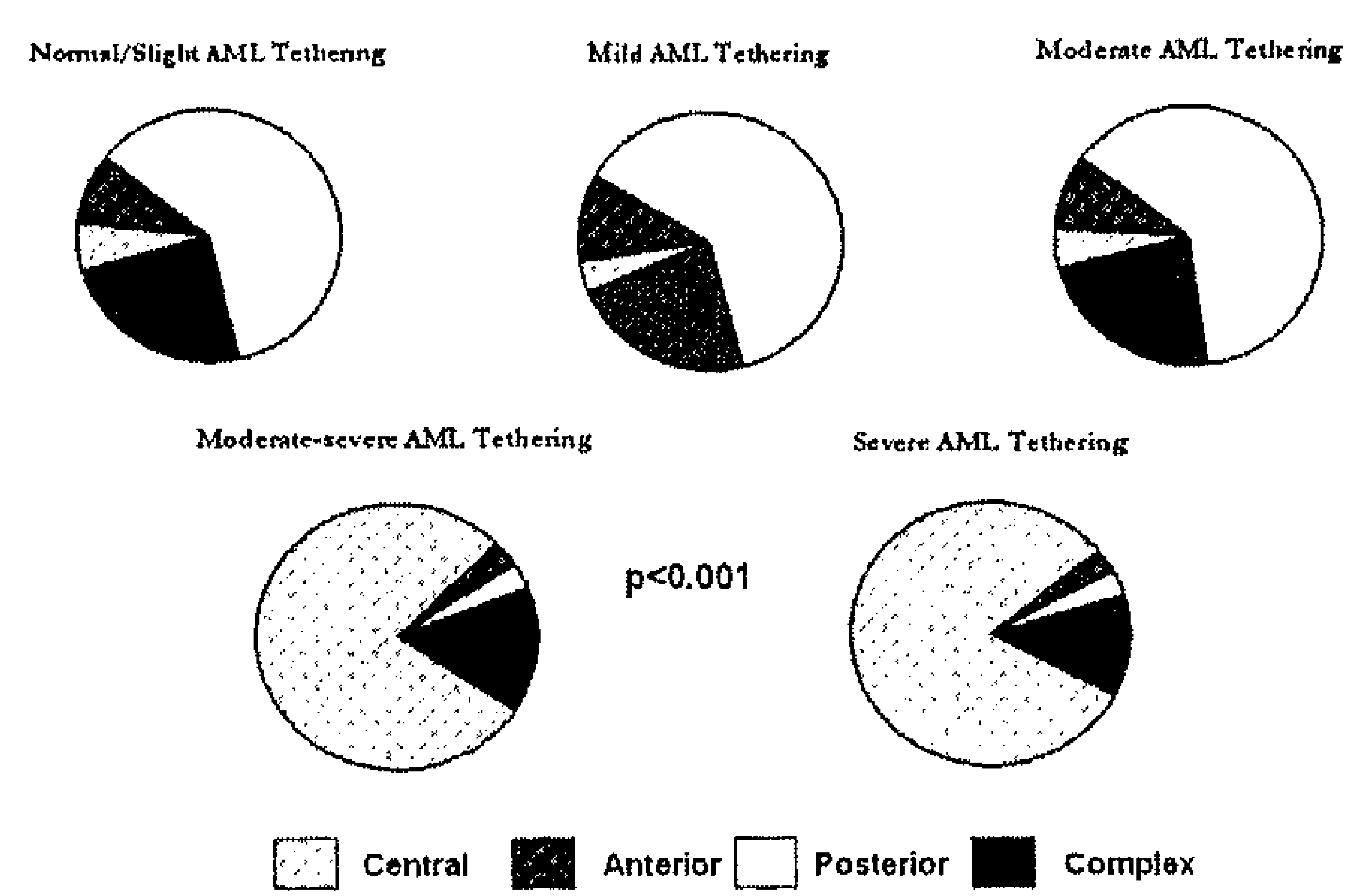

Postoperative Regurgitant Jet

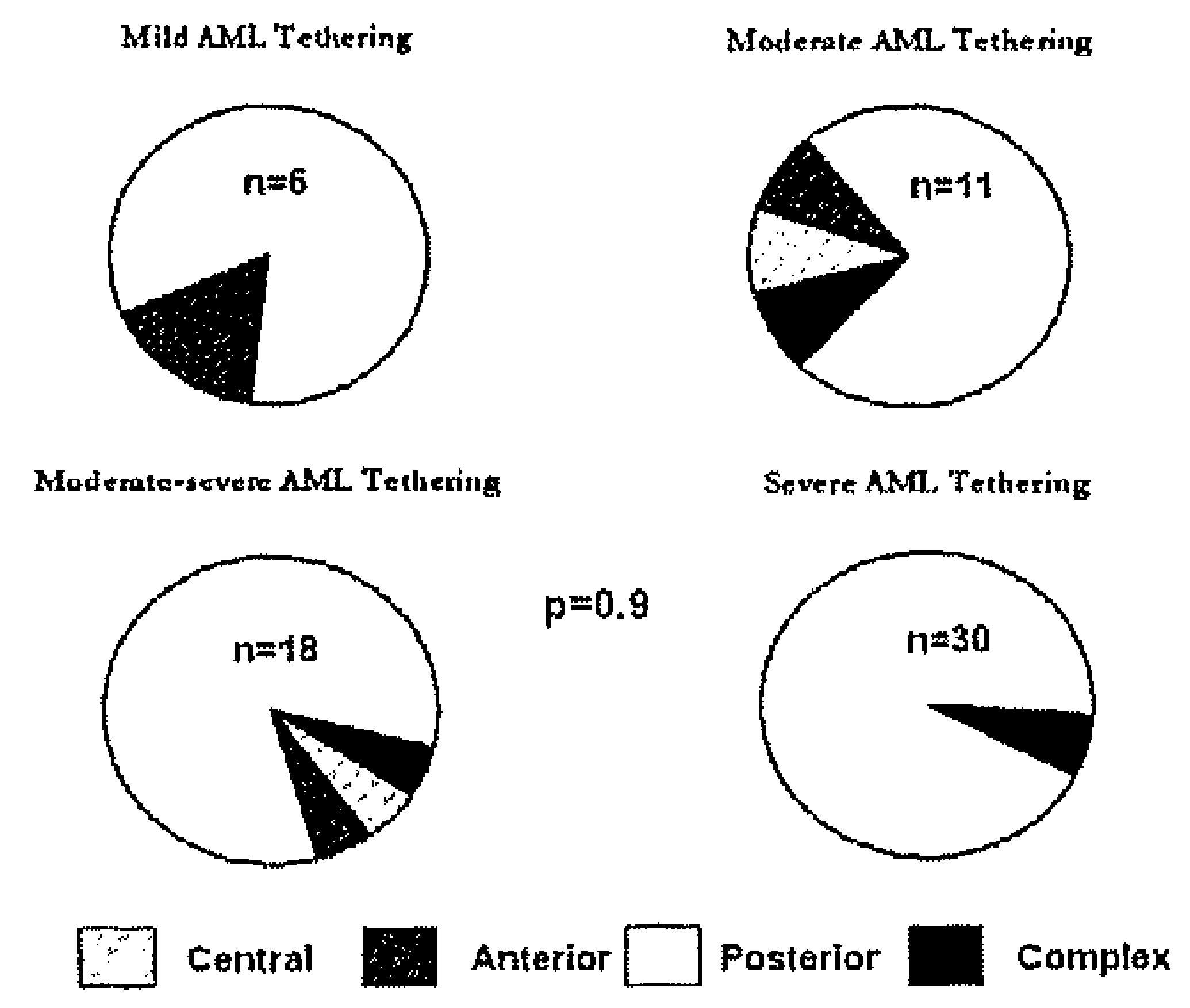

Figure 2. Regurgitant jet direction. (A) In the preoperative period, the regurgitant jet was predominantly posterior in patients in Group $1-3$, whereas it was mainly central in those with moderate-severe / severe anterior mitral tethering. (B) In patients with recurrent mitral regurgitation at follow up, the jet direction was posterior in most subjects without differences between Groups. 


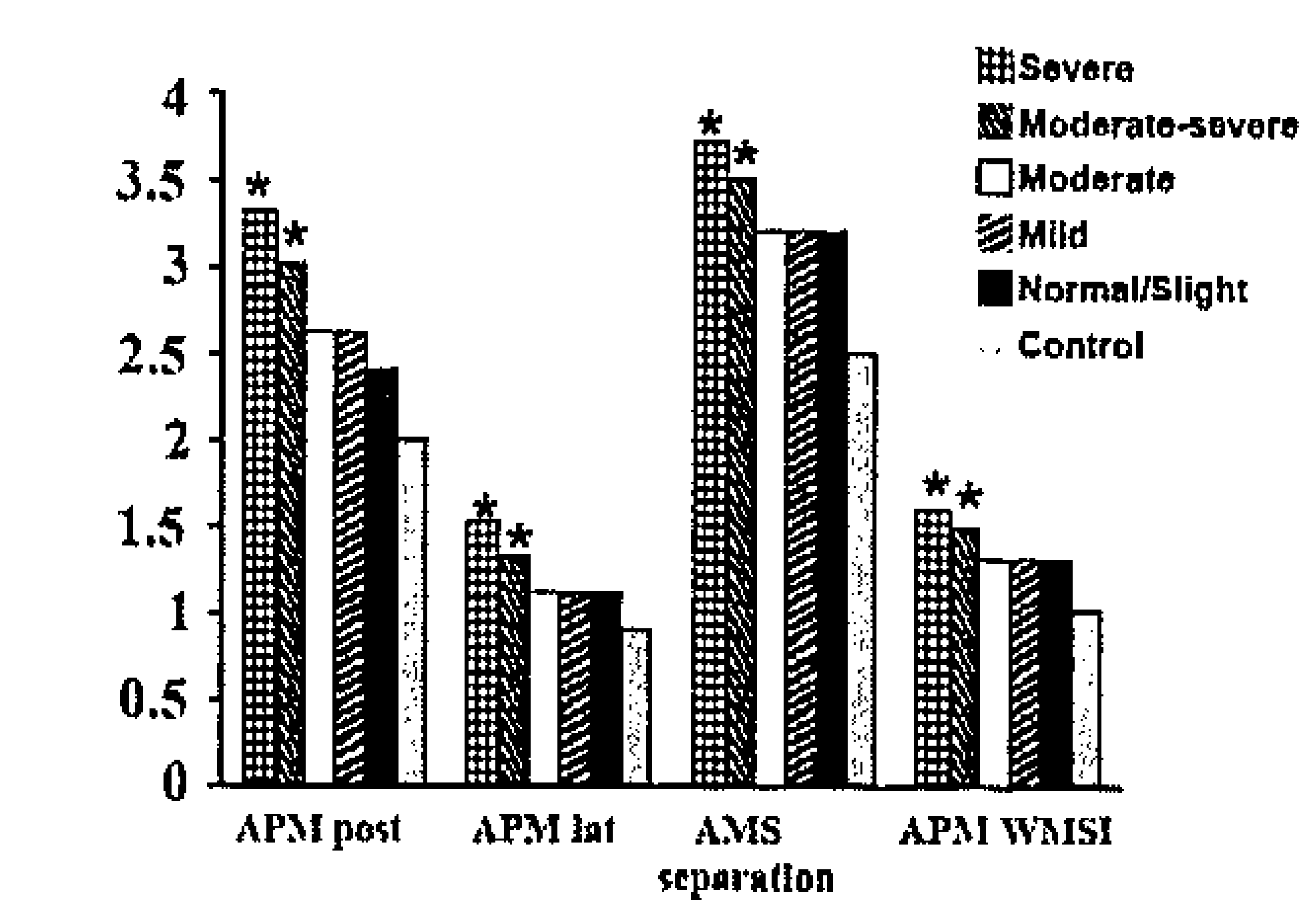

A

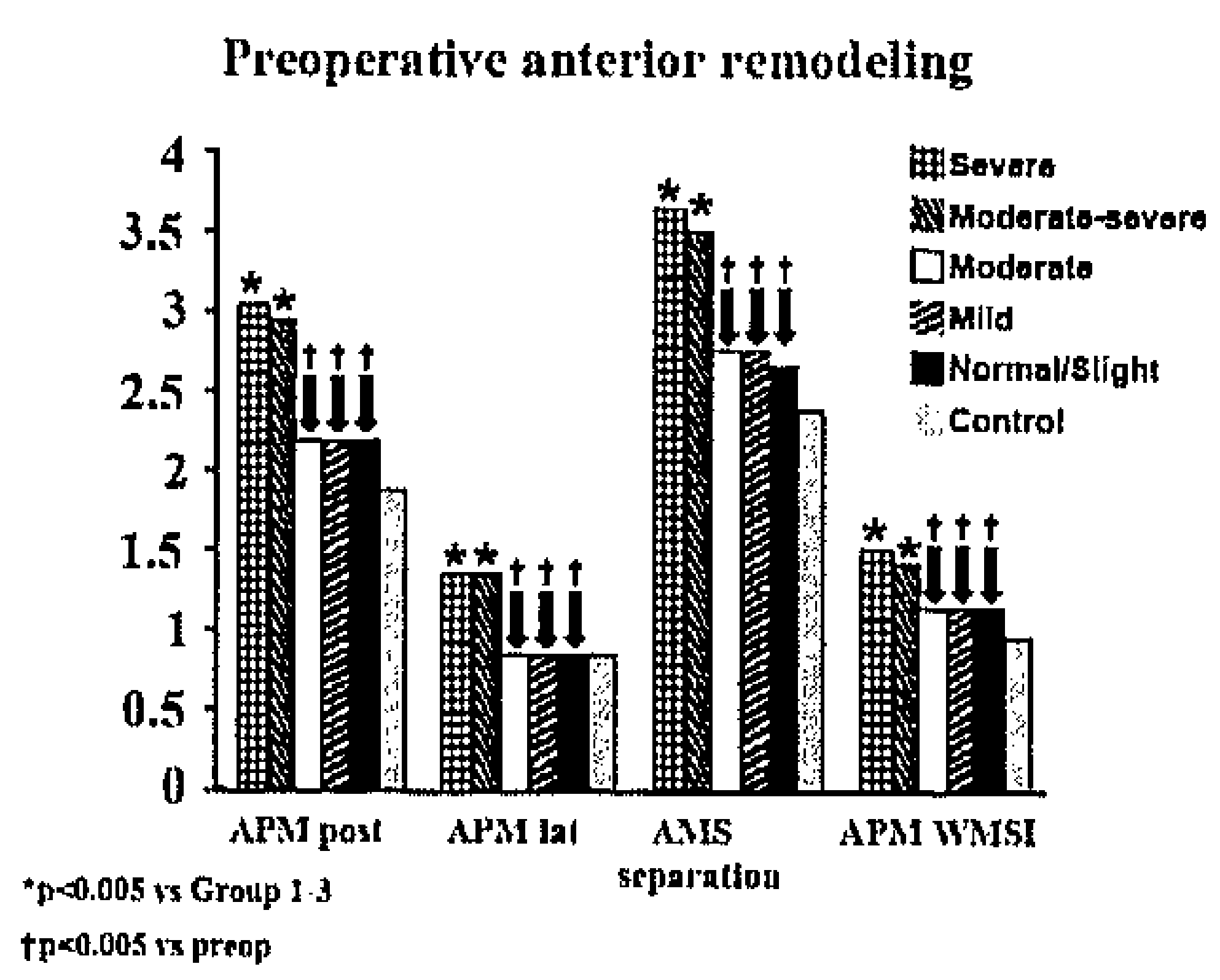

C

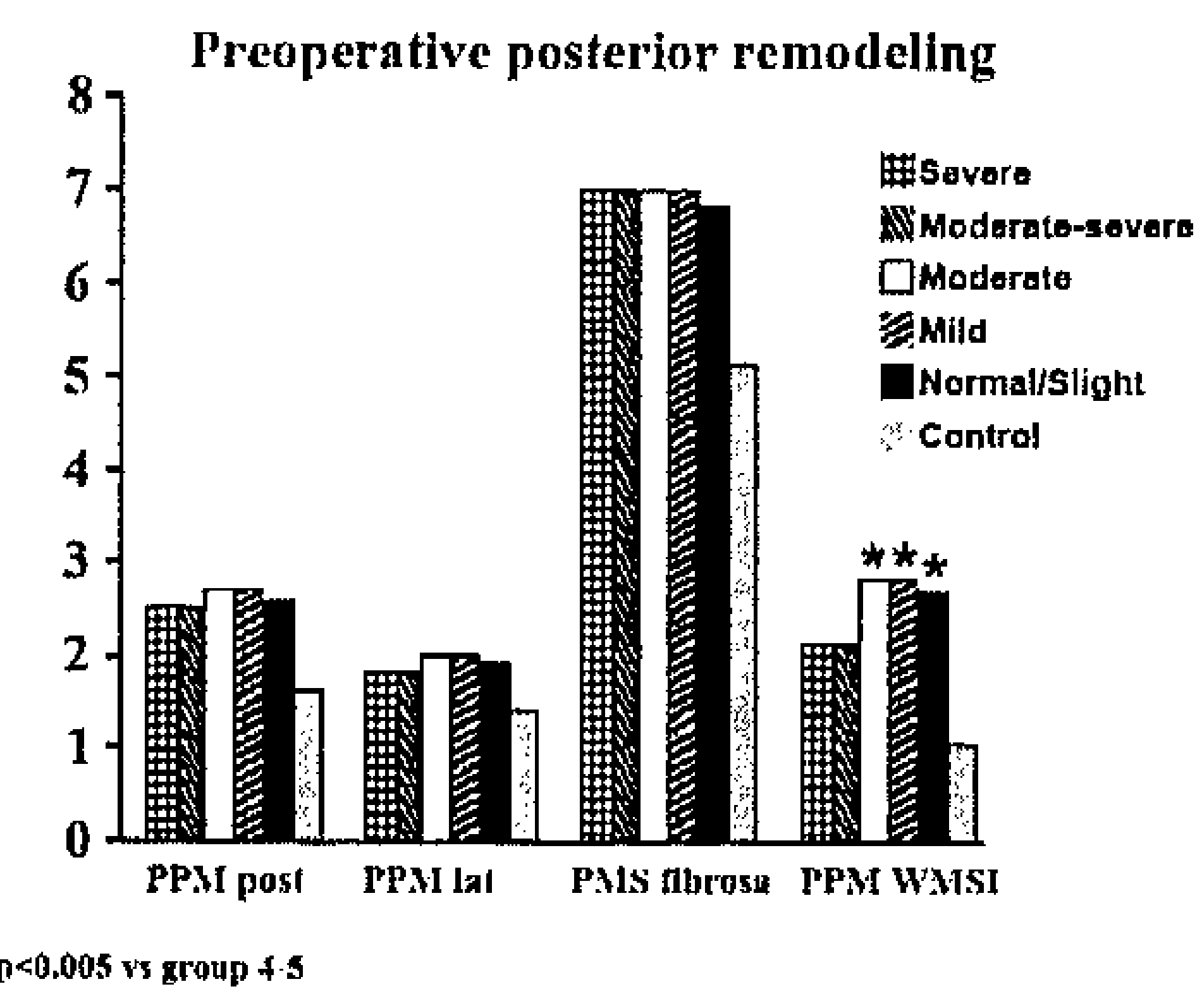

B

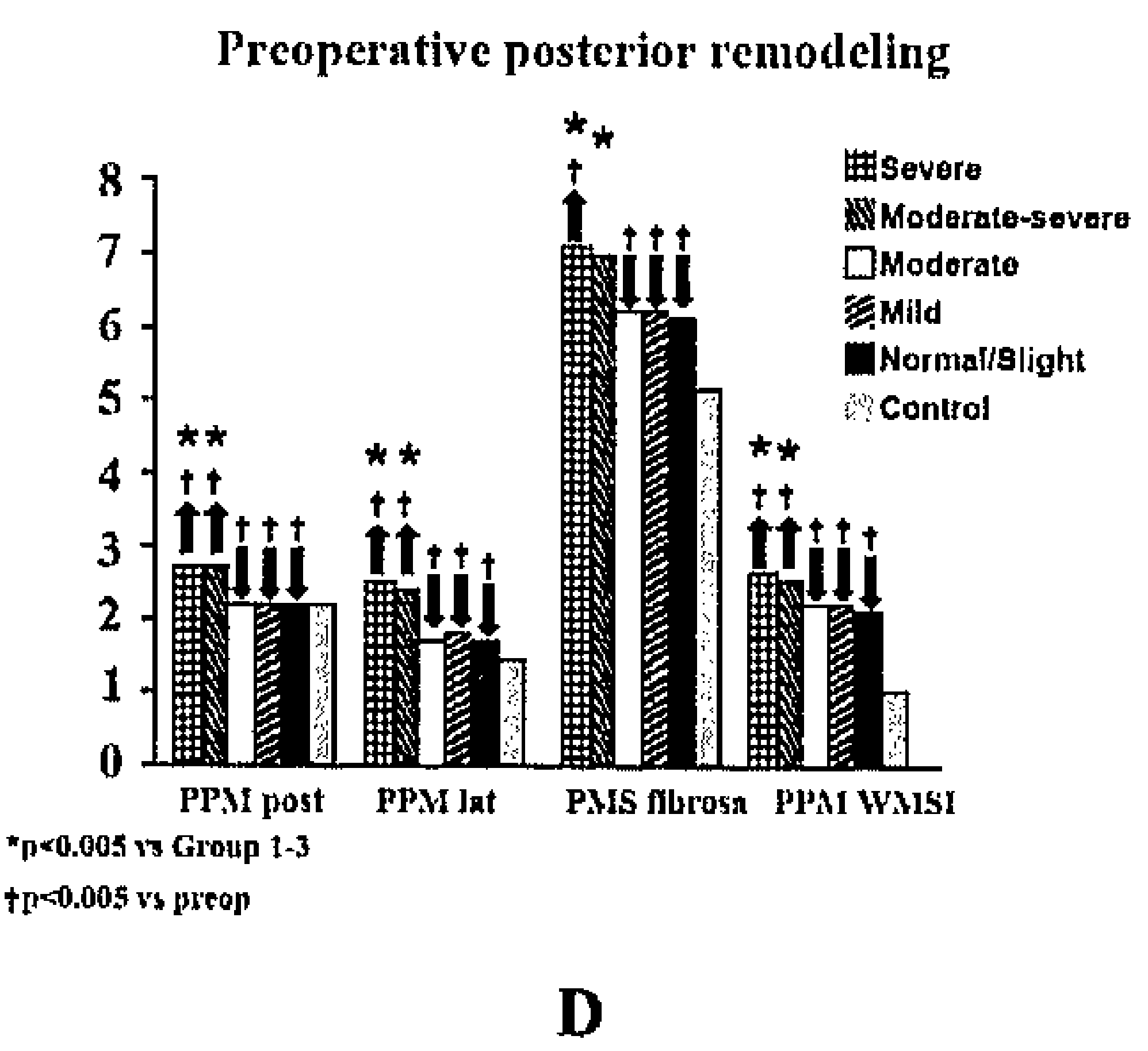

Figure 3. Papillary muscle displacement. (A) Patients with moderate-severe/severe anterior mitral leafler (AML) tethering had, at preoperative control greater posterior and lateral displacement of the anterior papillary muscle (APM post and APM lat, respectively) a wider papillary muscle separation (PMS) and a larger wall motion score index of the segments underlying the anterior papillary muscle (APM WMSI). (B) The wall motion score index of the segments underlying the posterior papillary muscle (PPM WMSI) was higher in patients in Groups 1-3. (C) At follow up APM post, APM lat, PMS and APM WMSI reduced significantly from the preoperative values only in Groups with AML tethering $\leq$ moderate. (D) PPM post, PPM lat, PMS fibrosis and PPM WMSI significantly decreased in patients with AML tethering $\leq$ moderate, whereas all these variables increased in patients with $\geq$ moderate-severe AML tethering at follow up control.

$(p=0.06)$. Differently, the bending angle $\gamma$ significantly increased at follow-up in patients with AML tethering smoderate; in contrast, the $\gamma$ showed a significant decrease in patients with moderate severe/severe AML tethering $(p<0.01)$. The posterior leaflet tethering angle $\beta^{\prime}$ (Figure 4D) increased whereas the excursion angle $\beta_{\mathrm{EX}}$ reduced at follow-up without differences between Groups (both, $\mathrm{p}<0.001$ ).

Finally, when the degree of tethering was examined further in the Groups, it was more symmetric in Groups $4-5$ before surgery (Figure 5A). However it became progressively more asymmetric at follow up control in all Groups (Figure 5B). 

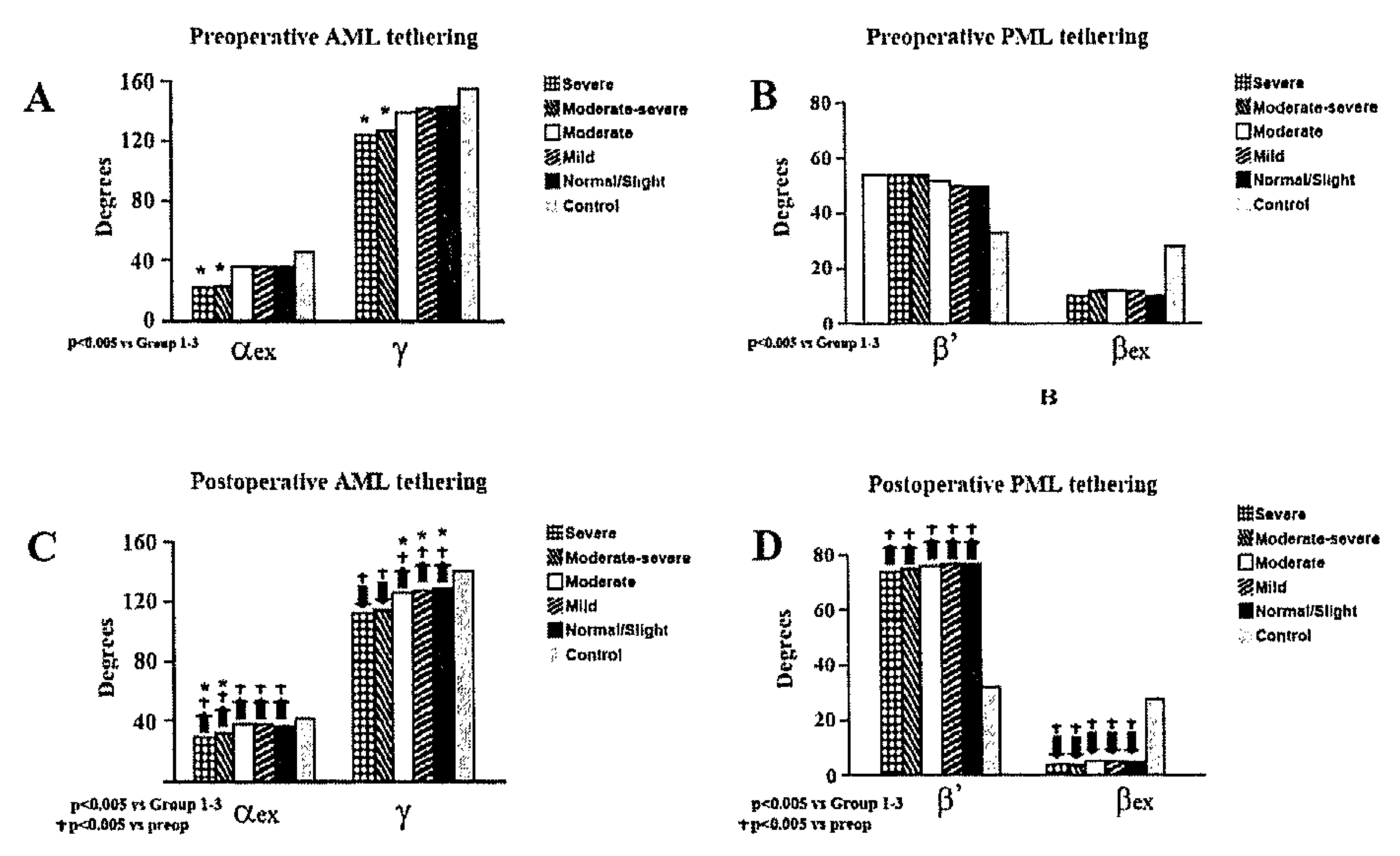

Figure 4. Mitral leaflet tethering. (A) At preoperative echocardiography the excursion angle of the anterior mirral leaflet $\left(\alpha_{E x}\right)$ and the bending angle $\gamma$ were lower in with $\geq$ moderate-severe AML tethering. $(B)$ The tethering angle and the excursion angle of the posterior mitral leaflet ( $\beta^{\prime}$ and $\beta_{\text {ex. }}$ respectively) were not different between Groups. (C) At follow up $\alpha_{E X}$ increased (Figure $4 \mathrm{C}$, in patients with $\geq$ moderate-severe AML tethering whereas $\gamma$ significantly decreased in patients with AML tethering $\geq$ moderate and reduced in patients with greater AML tethering. $(D) \beta^{\prime}$ increased at follow up control whereas the $\beta_{\mathrm{EX}}$ reduced without differences between Groups.

\section{Association of AML tethering with MR Recurrence}

At multivariable regression analysis anterior tethering angle $\alpha^{\prime} \geq 39.5^{\circ}(\mathrm{OR}, 5.6$ [bias-corrected $95 \%$ CI 2.6- 6.6], $\mathrm{p}<0.001)$, an anterior/posterior tethering angle ratio $\alpha^{\prime} / \beta^{\prime} \geq 0.76$ (OR, 5.2 [bias-corrected $95 \%$ CI 2.7- 6.0], $\mathrm{p}<0.001$ ) an anterior leaflet excursion angle $\alpha_{\mathrm{Ex}} \leq 35^{\circ}$ (OR, 2.5 [bias-corrected $95 \%$ CI 1.2-3.4], $\mathrm{p}<0.001$ ) and a coaptation height $h \geq 11 \mathrm{~mm}$ (OR, 2.3 [bias-corrected 95\% CI 1.1- 3.2], $\mathrm{p}=0.008)$ were independent predictors of recurrent $\mathrm{MR}$. The model resulted to be reliable (Hosmer-Lemeshow test, $[\mathrm{H}-\mathrm{L}] \mathrm{p}=0.7$ ) and accurate (c-index $[\mathrm{c}]=0.8$ ).

After we accounted for other clinical and echocardiographic risk factors AML tethering angle (adjusted OR, 4.9 [bias-corrected 95\% CI 2.3-5.8, $\mathrm{p}<0.001$ ), anterior/posterior tethering angle ratio (OR, 4.5 [bias-corrected $95 \% \mathrm{CI}$ 3.3- 5.6], $\mathrm{p}<0.001)$ anterior leaflet excursion angle (OR, 2.0 [bias-corrected $95 \% \mathrm{CI} 1.5-3.3$ ], $\mathrm{p}=0.007)$ and preoperative coaptation height (OR, 1.8 [bias-corrected $95 \%$ CI 0.9 - 3.2], $\mathrm{p}=0.03)$ remained strong predictors of recurrent regurgitation $(\mathrm{H}-\mathrm{L}=0.7$, $\mathrm{c}=0.7)$. 


\section{A}

Preoperative tethering pattern

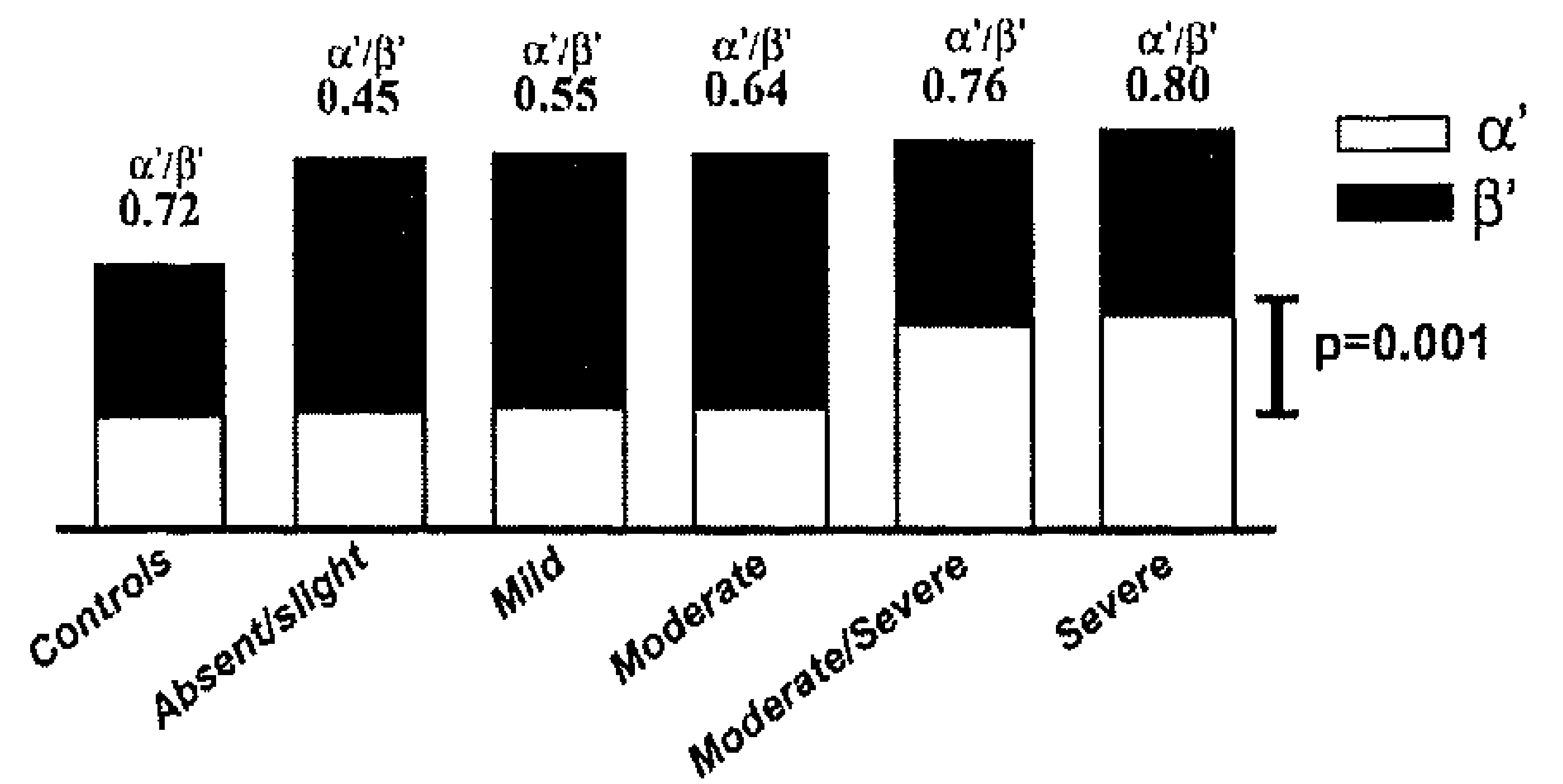

B

Postoperative tethering pattern

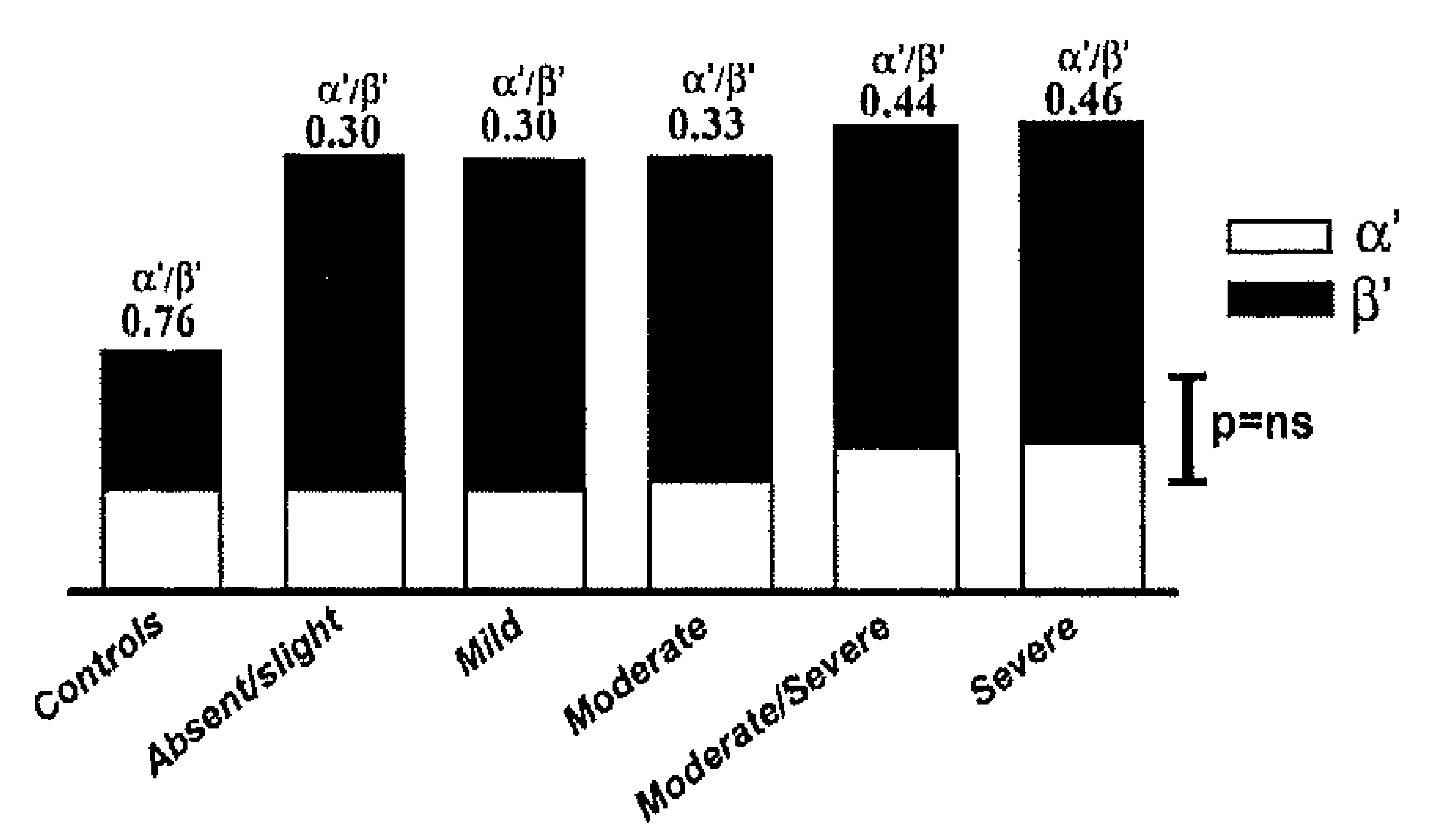

Figure 5. Tethering pattern. (A) In the preoperative period the tethering pattern was more symmetric in Groups $4-5$. (B) The tethering became progressively more asymmetric in all patients at follow up without significant differences berween Groups

Compared with patients with posterior leaflet tethering $\geq 45$ degrees $^{21}$ (adjusted OR 1 [bias-corrected 95\% CI 0.09-1.2), those with absent/slight or mild AML tethering had $22 \%$ and $76 \%$ higher odds for MR recurrence (adjusted $\mathrm{OR}_{s:} 1.22$ [bias-corrected $95 \% \mathrm{CI} 1.06-1.4$ ] and 1.76 [bias-corrected $95 \% \mathrm{CI} 1.2-1.9$ ], respectively). This rose to 2.5 times and 3 times higher MR recurrence in moderate (adjusted OR 2.5 [bias-corrected 95\% CI 1.7- 3.2) and moderate-severe (adjusted OR 3.0 [bias-corrected 95\% CI 2.1- 4.0) AML tethering, respectively. Finally, the increase in risk associated with severe AML tethering was > 4-fold (adjusted OR 4.2 [bias-corrected $95 \%$ CI 3.0-6.1)

When we allowed for interaction between AML tethering and other significant risk factors, the effect of AML tethering was generally attenuated in higher-risk patients (Table 4). Significant interactions were found between AML tethering and systolic sphericity index, end-systolic volume, myocardial performance index, wall motion score index, coaptation height and $\alpha^{\prime} / \beta^{\prime}$ ratio (each $\mathrm{p}<0.001$ ). 


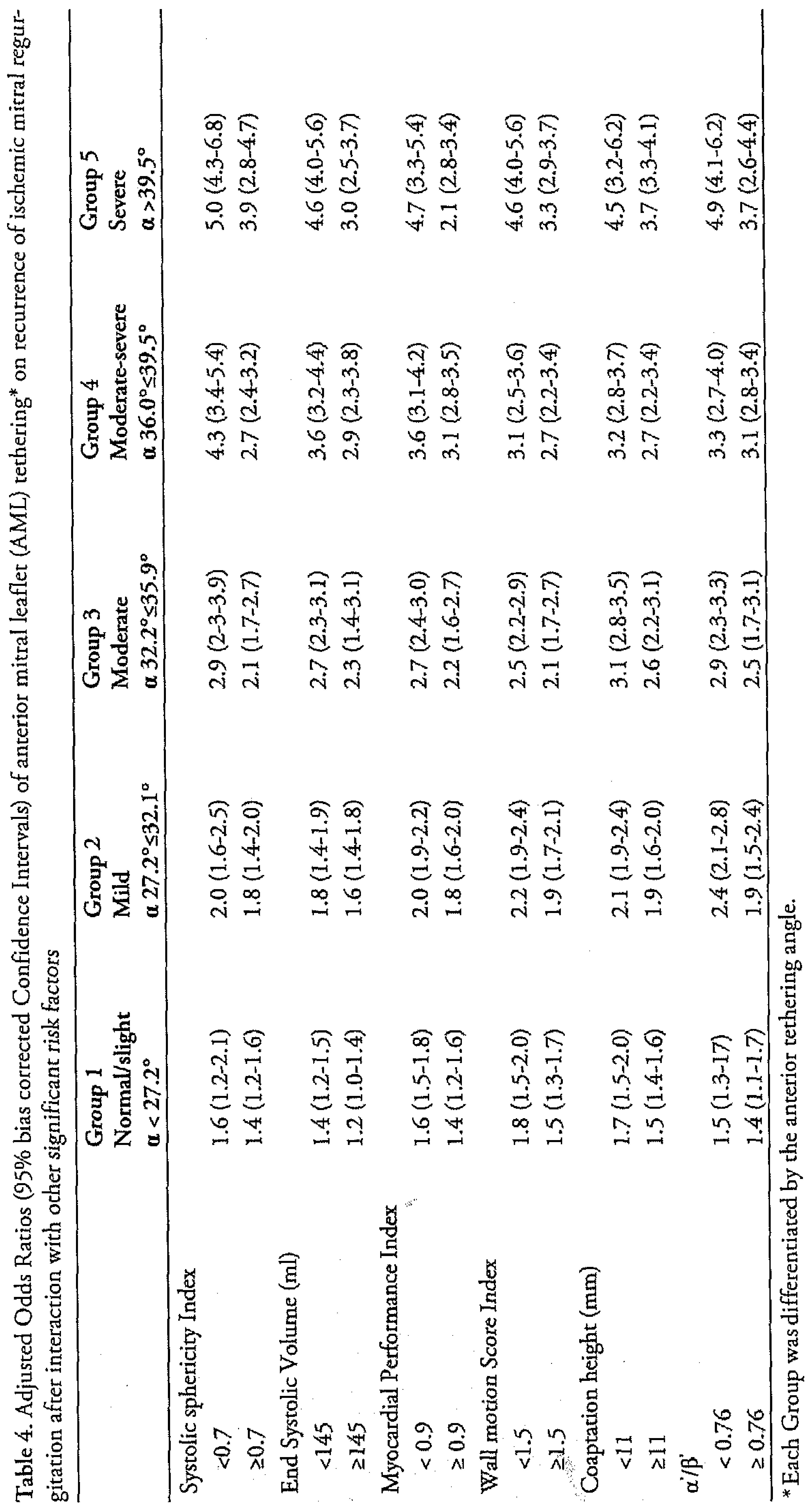




\section{DISCUSSION}

This report adds to a growing body of literature documenting recurrent ischemic mitral regurgitation after undersized mitral annuloplasty and coronary artery bypass grafting. The present study builds on these findings by examining the real impact of preoperative AML tethering on recurrence of MR after annuloplasty. Clinical data as well further treatment options offered for patients with discovery of recurrent MR have been previously reported ${ }^{7,20}$ and have not been included in the present study.

The main findings of this study can be summarized as follows:

First, preoperative assessment of AML tethering severity might be helpful to predict the recurrence of MR after undersized annuloplasty and coronary artery bypass grafting. To our knowledge our studies provide the first demonstration that MR recurrence can be predicted from the AML tethering pattern of the ischemic mitral valve on the preoperative echocardiographic evaluation. We previously demonstrated that preoperative AML tethering angle $\geq 39.5$ degrees had a very high sensitivity and specificity to predict recurrent MR after operation whereas the PML tethering was not significant ${ }^{20}$. Nonetheless, the influence of AML tethering could be secondary to other factors and the altered leaflet geometry and recurrent MR could represent both the result of abnormal left ventricular function and geometry which may be primary predictors. Therefore in this study multivariable models were adjusted for important risk factors of MR recurrence we had previously recognized ${ }^{7,20}$. After we accounted for other clinical and echocardiographic key risk factors AML tethering angle remained a strong predictor of recurrent regurgitation. Indeed patients with moderate or moderate-severe preoperative AML tethering showed a 2.5 times and 3 times higher Odds for MR recurrence compared to those with significant PML tethering (PML angle $\geq 45$ degrees $^{21}$ ). This rose to $>4$ fold in patient with severe AML tethering.

In contrast with our results, Kuwahara et a ${ }^{12}$ showed that increased PML tethering was the primary determinant of late MR in 30 patients undergoing surgical annuloplasty for ischemic mitral regurgitation. In addition, Magne and coworkers $^{21}$ identified a PML angle $\geq 45$ degrees (sensitivity $100 \%$, specificity $97 \%$ ) to be a predictor of postoperative persistence of MR after annuloplasty.

However, these authors studied recurrent/persistent and persistent MR, respectively, whereas in our study residual MR was excluded. Persistent /residual MR is presumably due to immediate failure of the technique employed rather than LV remodeling ${ }^{22}$ and it is not surprising that regurgitation persists at late follow up in these patients. Furthermore, Matsunaga and coworkers ${ }^{23}$ showed that the severity of the posterior papillary muscle displacement might be a predictor of ring annuloplasty failure. These authors, differently from us, employed in their patients a flexible ring (Duran ring, Medtronic, INC, Minneapolis, MN, US). In additions, they adopted the "PM angle" as an indirect measurement of tethering, defined as the 
angle formed by the mitral annular plane and the line connecting the PM tips to the mitral annulus. It can be influenced by changes in LV geometry and dimension. In contrast we measured the angles between the mitral leaflets and the annulus which reflect directly the excursion of the leaflet and their effective tethering.

Second, our findings confirmed that patients with more accentuated AML tethering cannot guarantee a sufficiently long anterior leaflet to cover the whole antero-posterior diameter and this is worsened by the augmented tethering of the posterior leaflet occurring postoperatively in all patients which further displaces the coaptation point. In other words, the AML must be sufficiently long and mobile to ensure a proper surface of coaptation. In our experience, a coaptation length (CL) $\geq 8 \mathrm{~mm}$ at the end of the procedure guaranteed annuloplasty durability. Coaptation length was at any time lower in Groups $4-5(\mathrm{p}<0.001)$ which showed $100 \%$ patients with $\mathrm{CL} \leq 8 \mathrm{~mm}$ at follow-up.

Third, patients with higher preoperative AML tethering had a greater local LV remodeling of the segments of the anterior papillary muscle, a greater lateral and posterior displacement of the anterior papillary muscle and a wider PMs separation and a more symmetric tethering pattern. However patients with postoperative MR showed a mechanism different from that of MR at baseline, with a tethering pattern becoming progressively more asymmetric. This is due to continued remodeling that is mainly posterior in the postoperative period, whereas the LV remodeling in the area of the AML and, consequently, the anterior leaflet tethering, is maintained as in the preoperative situation.

These findings support previous clinical results reported by Agricola et a $1^{15}$ who described two Groups of patients with CIMR: likewise patients with more pronounced AML tethering, their symmetric Group had prevalent posterior and lateral displacement of anterior papillary muscle, PMs separation and WMSI of the regions supporting the anterior papillary muscle.

Fourth, continued LV remodeling occurs in all Groups in the postoperative period and it predominantly involves the region of the LV supporting the posterior papillary muscle. There was no continued anterior remodeling as demonstrated by wall motion score index of segments underlying the anterior papillary muscle which remained constant over time postoperatively in the entire patient cohort. This is in accordance with published data ${ }^{10,24}$ and confirms that tethering of the posterior leaflet may be exacerbated by annuloplasty which, reducing annular size, can potentially shift the posterior annulus farther anteriorly, increasing its displacement relative to the papillary muscles without displacing the anterior annulus fixed at the aortic root ${ }^{11}$. 
Anterior mitral leaflet tethering, irrespective of LV function and geometry, is a key factor to ensure durable MV-plasty. The possibility of allowing both leaflets into apposition depends on the ability of AML to move toward the annulus and to reach the posterior one. If this movement is insufficient the mitral leaflets never coapt no matter how much posterior annulus is reduced. The angle $\alpha$ ' gives an accurate measure of AML tethering. Furthermore, the length of the AML can be estimated by the coaptation height which represents the depth of the AML during systole, in our opinion and according to Calafiore et $\mathrm{a}^{25}$, when it is more than $11 \mathrm{~mm}$, it is associated with a high risk of MR recurrence after reductive annuloplasty.

Thus we believe that in patients with severe $\left(\alpha^{\prime} \geq 39.5^{\circ}\right)$ or moderate-severe $\left(\alpha^{\prime}\right.$ $36.0^{\circ} \leq 39.5^{\circ}$ ) AML with a coaptation height $>11 \mathrm{~mm}$, the risk of performing UMRA must be carefully weighed against those risk associated with a chordal sparing mitral valve replacement.

It is arguable if patients with a more accentuated AML tethering and restrictive excursion could benefit from anterior basal chord cutting as a method of improving leaflet mobility and decreasing leaflet tethering. Nonetheless we have no experience with this technique and this issue deserves further investigation.

Another interesting point is that, in our experience, a long anterior leaflet was not always sufficient to balance tethering forces secondary to postoperative remodeling. Indeed, in a subgroup of patients with mild or moderate AML tethering who showed recurrent MR, the coaptation length was small $(2.6 \mathrm{~mm})$ or completely absent, without any apposition surface and with the AML which closed against the annulus. Thus we believe that coaptation length (CL) gained at the end of procedure plays a significant role in preventing MR recurrence and in all subjects a minimum CL of $8 \mathrm{~mm}$ should be achieved, as suggested by other authors ${ }^{25,26}$.

\section{LiMitations OF THE STUDY}

There were several limitations of the present study. First, a viability testing was not performed in these patients. Therefore recurrence of $M R$ might be also due to irreversible ischemic myocardial damage (not-viable myocardium). Furthermore, in relation to the papillary muscle displacement data presented, the revascularization of viable regions related to papillary muscle function might be the most important predictor of success with this operation. However, this limitation is shared with most of published papers and this issue deserves further investigation.

Second, evaluation of mechanisms of recurrent ischemic mitral regurgitation was based on geometric assumptions and $2 \mathrm{D}$ echocardiographic measurements which are subject to image-plane positioning errors. Hence it might be not accurate in shape distorted post-infarction left ventricles. The era of 3-D echocardiography 
has started potentially leading to new insights into LV remodeling, mitral apparatus shape and function ${ }^{27}$. Nonetheless the 3-D method is still on its way and is far for being validated, standardized and widely applied in the clinical practice ${ }^{28}$.

Third, the issue of annulus re-shaping during annuloplasty has not been addressed. We employed only rigid/semi-flexible plane annuloplasty rings which may flatten the natural saddle shape causing greater tension on chordae and leaflets. Innovative annular rings have been recently introduced in the market which mimic the shape of healthy mitral annulus ${ }^{29,30}$. This is object of an ongoing study.

Fourth, postoperative evaluation of the coronary status was not assessed. It would have been helpful to differentiate between surgical failure (valve repair and $\mathrm{CABG}$ ) and the progress of the coronary disease.

\section{CONCLUSIONS}

This study has demonstrated that preoperative anterior mitral leaflet tethering is a powerful predictor of MR recurrence after annuloplasty, independently of LV function, dimensions and geometry. Measures of leaflet tethering should be incorporated into risk prediction model for unsuccessful MV plasty and might represent fundamental findings to identify ischemic patients who can really benefit from this kind of surgery. Further larger studies are necessary to confirm our findings.

\section{REFERENCES}

1. Bolling SF, Pagani FD, Deeb GM, Bach DS.Intermediate term outcome of mitral reconstruction in cardiomyopathy.J Thorac Cardiovasc Surg 1998;115(2):381-6.

2. Gillinov AM, Wierup PN, Blackstone EH, et al.Is repair preferable to replacement for ischemic mitral regurgitation?] Thorac Cardiovasc Surg. 2001; 122: 1125-41.

3. Miller DC.Ischemic mitral regurgitation redux--to repair or to replace?] Thorac Cardiovasc Surg. 2001;122(6):1059-62.

4. Grossi EA, Goldberg JD, LaPietra A, et al.Ischemic mitral valve reconstruction and replacement: comparison of long-term survival and complications.J Thorac Cardiovasc Surg.2001;122(6):110724.

5. Adams DH, Filsoufi F, Aklog L.Surgical treatment of the ischemic mitral valve.J Hearr Valve Dis 2002;11 Suppl 1:S21-5.

6. Bolling SF, Pagani FD, Deeb GM, Bach DS.Intermediate term outcome of mitral reconstruction in cardiomyopathy. J Thorac Cardiovasc Surg 1998;115(2):381- 6.

7. Gelsomino S, Lorusso R, De Cicco G, et al.Five-year echocardiographic results of combined undersized mitral ring annuloplasty and coronary artery bypass grafting for chronic ischaemic mitral regurgitation. Eur Heart J. 2008;29(2):231-40. 
8. Serri K, Bouchard D, Demers P, et al.Is a good perioperative echocardiographic result predictive of durability in ischemic mitral valve repair?] Thorac Cardiovasc Surg 2006;131:523-529.

9. McGee EC, Gillinov AM, Blackstone EH, et al.Recurrent mitral regurgitation after annuloplasty for functional ischemic mitral regurgitation.J Thorac Cardiovasc Surg 2004, 128(6):916-24.

10. Hung J, Papakostas L, Tahta SA, et al.Mechanism of recurrent ischemic mitral regurgitation after annuloplasty: continued LV remodeling as a moving target.Circulation 2004;110 (Suppl. II):II85-II90

11. Green GR, Dagum P, Glasson JR, et al. Restricted posterior leaflet motion after mitral ring annuloplasty.Ann Thorac Surg1999;68(6):2100-6.

12. Kuwahara E, Otsuji $Y$, Iguro $Y$, et al.Mechanism of recurrent/persistent ischemic/functional mitral regurgitation in the chronic phase after surgical annuloplasty: importance of augmented posterior leaflet tethering:Circulation2006 4;114(1 Suppl):1529-34.

13. Gelsomino S, Lorusso R, Caciolli S, et al. Insights on Left Ventricular and Valvular Mechanisms of Recurrent Ischemic Mitral Regurgitation After Restrictive Annuloplasty and Coronary Artery Bypass Grafting. J Thorac Cardiovasc Surg 2008;136(2): 507-18.

14. Bland MJ, Altman DJ.Statistical method for assessing agreement between two methods of clinical measurements.Lancet 1986;1:307-310.

15. Agricola E, Oppizzi M, Maisano F et al.Echocardiographic classification of chronic mitral regurgitation caused by restricted motion according to tethering pattern. Eur JEchocardiography 2004;5:326-34.

16. Romesburg HC. Cluster analysis for researchers. North Carolina: Lulu Press;2004:1-330.

17. Harrel FE., Lee KL, Califf RM, Pryor DB, Rosati RA.Regression modeling strategies for improved prognostic prediction. Statistics in Medicine 1984;3:143-52.

18. Harrel FE Jr, Lee KL, Mark DB. Multivariable Prognostic Models:Issues in developing models, evaluating assumptions and adequacy, and measuring and reducing errors. Stats Med 1996:36187.

19. Hosmer DW, Lemeshow S. Applied Logistic regression. $2^{\text {nd }}$ ed.New York: Wiley-Interscience; 2000:143-202.

20. Gelsomino S, Lorusso R, Capecchi I, et al.Left ventricular reverse remodeling after undersized mitral ring annuloplasty in patients with ischemic regurgitation.Ann Thorac Surg. 2008;85(4):1319-30.

21. Magne J, Pibarot P, Dagenais F, Hachicha Z, Dumesnil JG, Senechal M. Preoperative posterior leaflet angle accurately predicts outcome after restrictive mitral valve annuloplasty for ischemic mitral regurgitation.Circulation.2007;115(6):782-91.

22. Adams DH, Anyanwu AC. Pitfalls and limitations in measuring and interpreting the outcomes of mitral valve repair. J.Thorac Cardiovasc Surg 2006;131:523-9.

23. Matsunaga A, Tahta SA, Duran CMG.Failure of reduction annuloplasty for functional ischemic mitral regurgitation.J Heart Valve Dis2004, 13:390-8.

24. Zhu F, Otsuji Y, Yotsumoto G, et al. Mechanism of persistent ischemic mitral regurgitation after annuloplasty: importance of augmented posterior mitral leaflet tethering.Circulation. 2005;112(Suppl):I396-401. 
25. Calafiore AM, Di Mauro M, Gallina S, et al. Mitral valve surgery for chronic ischemic mitral regurgitation.Ann Thorac Surg.2004;77(6):1989-97.

26. Braun J, Bax JJ, Versteegh MI, et al.Preoperative left ventricular dimensions predict reverse remodeling following restrictive mitral annuloplasty in ischemic mitral regurgitation.Eur J Cardiothorac Surg.2005;27(5):847-53.

27. Tsukiji M, Watanabe N, Yamaura Y, et al. Three-Dimensional Quantitation of Mitral Valve Coaptation by a Novel Software System with Transthoracic Real-Time Three-Dimensional Echocardiography.J Am Soc Echocardiogr.2008;21(1):43-6.

28. Ryan LP, Jackson BM, Parish LM, et al. Mitral valve tenting index for assessment of subvalvular remodeling.Ann Thorac Surg.2007;84(4):1243-9.

29. Votta E, Maisano F, Bolling SF, et al.The Geoform disease-specific annuloplasty system: a finite element study. Ann Thorac Surg. 2007;84(1):92-101.

30. Jimenez JH, Liou SW, Padala $M$, et al. A saddle-shaped annulus reduces systolic strain on the central region of the mitral valve anterior leaflet.] Thorac Cardiovasc Surg. 2007;134(6):1562-8. 
APPENDIX 1

Bland -Altman limits of agreement for intra-observer and inter-observer variability.

\begin{tabular}{lllll}
\hline Variable & & $\begin{array}{l}\text { Mean } \\
\text { difference }\end{array}$ & $\begin{array}{l}\text { Standard } \\
\text { deviation }\end{array}$ & $\begin{array}{l}\text { 95\% limits } \\
\text { of agreement }\end{array}$ \\
\hline$\alpha^{\prime}\left(^{\circ}\right)$ & Intra-observer (IC) & 1.4 & 1.9 & $-2.2-5.1$ \\
& Intra-observer (CR) & 1.7 & 1.8 & $-1.8-5.2$ \\
& Inter observer & 1.8 & 2.1 & $-2.3-6.0$ \\
$\beta^{\prime}\left(^{\circ}\right)$ & Intra-observer (IC) & 2.1 & 2.2 & $-2.2-6.4$ \\
& Intra-observer (CR) & 1.5 & 2.2 & $-2.8-5.9$ \\
& Inter observer & 1.4 & 3.5 & $-5.5-8.3$ \\
$\mathrm{~h}(\mathrm{~mm})$ & Intra-observer (IC) & 0.1 & 0.3 & $-0.4-0.7$ \\
& Intra-observer (CR) & 0.1 & 0.2 & $-0.4-0.6$ \\
& Inter observer & 0.2 & 0.4 & $-0.5-1.0$ \\
\hline
\end{tabular}

Intra-observer and inter-observer relative differences were $<5 \%$ for all parameters. The Bland-Altman method showed excellent agreement between intra-observer and inter-observer measurements in both low and high values of echocardiographic parameters. Observers: observer 1: Irene Capecchi, (IC); observer 2: Carlo Rostagno (CR).Inter-observer (observer 1-observer 2).

Abbreviations: $\alpha^{\prime}=$ Anterior mitral leaflet tethering angle, $\beta^{\prime}=$ Posterior mitral leaflet tethering angle; $h$ : coaptation height;. 


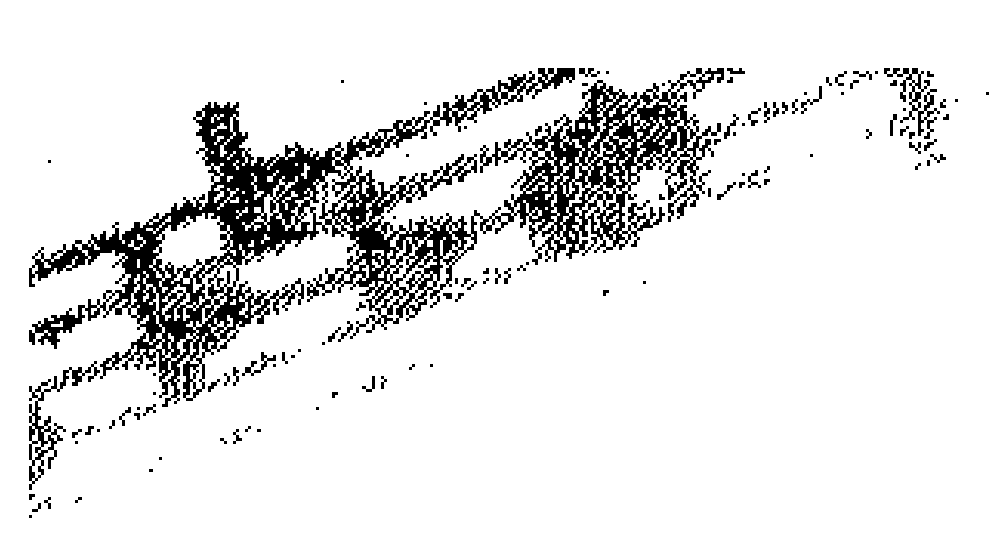

6

Left ventricular diastolic function after restrictive mitral ring annuloplasty in chronic ischemic mitral regurgitation and its predictive value on outcome and recurrence of regurgitation

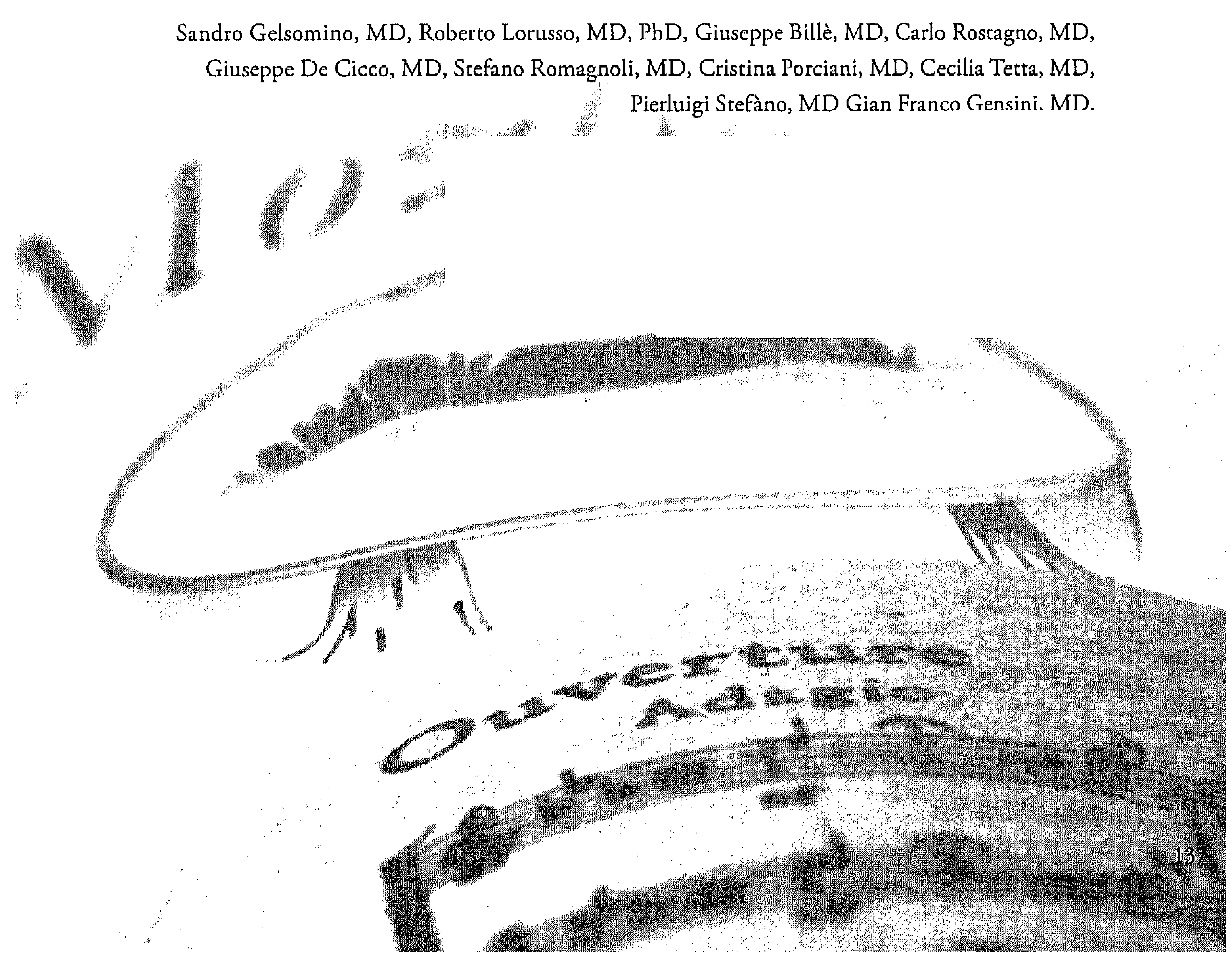




\section{ABSTRACT}

Background. This study was aimed at exploring the predictive value of diastolic function on clinical outcome and recurrence of ischemic mitral regurgitation following combined undersized mitral annuloplasty (UMRA) and coronary artery bypass grafting (CABG). Methods. Two hundredthirty-four patients with chronic ischemic mitral regurgitation (CIMR) who survived combined UMRA and CABG between September 2001 and September 2007, were divided into four groups on the basis of baseline Deceleration Time (DT) and systolic-diastolic pulmonary venous flow ratio (S/D): Group 1, normal ( $n=48)$, Group 2, impaired relaxation ( $n=61)$, Group 3, pseudonormal $(n=60)$ and Group 4, restrictive $(n=65)$. Echocardiograms were performed, preoperatively, at discharge and at follow-up appointments (early, 6 months [interquartile range, IQR] 3-8 months; late, 38 months [IQR 17-53 months]). Results. Early mortality rate was highest in the restrictive group $(9.2 \%, p<0.001)$. In addition 6-year actuarial survival was significantly lower in Group $4(p=0.025)$. Ar late follow-up, among patients in group 4, 58.4\% $(\mathrm{n}=38)$ had an MR grade $\geq 2(\mathrm{p}<0.001)$. Furthermore, DT $<140 \mathrm{~ms}$ and $S / D<0.80$ were independent predictors of early ( $\mathrm{p}<0.001$ and 0.004 , respectively) and late (both $\mathrm{p}<0.001)$ death. Finally DT $<140 \mathrm{~ms}$ was the only diastolic independent predictor of MR recurrence $(\mathrm{p}<0.001)$. Conclusions. In patients with CIMR undergoing combined CABG and UMRA restrictive LV diastolic filling pattern is an important preoperative marker of high early and late death and recurrence of MR. 


\section{INTRODUCTION}

Chronic Ischemic mitral regurgitation (CIMR) is a functional insufficiency of the mitral valve related to changes either in the left ventricle (LV) or valve geometry and performance, resulting from ischemic heart disease ${ }^{1-2}$. The incidence of MR following myocardial infarction (MI) is approximately $20 \%$ and is higher in patients with previous inferior $\mathrm{MI}^{3,4}$. In subjects with coronary artery disease and concomitant ischemic LV dysfunction, MR is frequently found with notably $30 \%$ presenting severe $M R^{5}$.

A standard surgical approach to relieve CIMR is undersized mitral ring annuloplasty (UMRA) aimed at improving both leaflet malcoaptation and LV shape (LV reverse remodeling, LVRR) $)^{6,7}$.

Systolic dysfunction is an important predictor of early mortality and poor longterm outcome after combined mitral valve repair and coronary artery bypass grafting $(\mathrm{CABG})^{8}$. Nonetheless, little is known about diastolic function and its influence on outcome and LVRR.

We sought to investigate the predictive value of preoperative impairment of diastolic function on early and late outcome and recurrence of mitral regurgitation in patients with Chronic ischemic mitral regurgitation (CIMR) undergoing combined UMRA and CABG.

\section{METHODS}

\section{Definitions}

CIMR was defined as the combination of mild-to-severe MR with 1) Prior myocardial infarction (MI) $>16$ days; 2) seventy-five percent or greater stenosis of at least one coronary vessel; 3) a corresponding regional wall motion abnormality; 4) type IIIb leaflet dysfunction following Carpentier's classification ${ }^{9}$ with or without annular dilatation.

Exclusion criteria were: 1) degenerative or other non-ischemic etiology; 2) ischemic isolated type I or type II dysfunction"; 3) additional mitral valve repair procedures; 4) other valvular or congenital heart diseases; 5) previous cardiac surgery/percutaneous transluminal coronary angioplasty; 6) atrial fibrillation or sinus thythm with heart rate at rest $>100 \mathrm{bts} / \mathrm{min}$.

\section{Patients population}

Among 270 Subjects with CIMR undergoing combined CABG and UMRA in our Institution (Cardiac Surgery, Careggi Hospital, Florence, Italy) between 
September 2001 and September 2007 and prospectively enrolled in the study, 36 were excluded: 4 had intraoperative annuloplasty failure, 12 showed residual MR (insufficiency $\geq 2+$ following valve repair documented at discharge) and 20 had incomplete data. The study population consisted of 234 patients. Clinical follow up information was obtained from all survivors through outpatient visits and phone calls and was $100 \%$ complete. Median early follow up was 6 months (interquartile range [IQR] 3-8), median late follow up was 38 months (IQR 17-53).

Following the World Medical Association guidelines concerning ethical principles for medical research involving human subjects, the study was approved by Institutional Ethics Board. Furthermore and all patients gave their informed consent. The authors had full access to the data and take responsibility for its integrity. All authors have read and agreed to the manuscript as written.

\section{SURGERY}

All patients underwent associated coronary artery bypass grafting. For the purpose of this study, complete revascularization was accomplished when, at least one graft was placed distal to an approximately $50 \%$ diameter narrowing in each of the three major vascular system in which arterial narrowing of this severity was noted in a vessel $\geq 1.5 \mathrm{~mm}$ of diameter. It was not considered necessary to bypass all obstructed diagonal branches of the anterior descending or marginal branches of the circumflex coronary arteries for a classification of complete revascularization. Following this definition $100 \%$ patients underwent complete revascularization.

The ring size was determined by standard measurements of the inter-trigonal distance and anterior leaflet height. A downsizing by two ring sizes was performed in all patients. After cardiopulmonary bypass (CPB), a transesophageal echocardiography (TEE) was performed to assess residual MR: leaflet coaptation $\geq 0.5 \mathrm{~cm}, \mathrm{MR}$ $\leq 1$ and systolic MV area $>2 \mathrm{~cm}^{2}$ was assessed as successful repair.

\section{Echocardiographic Study}

Echocardiographic studies were performed on Acuson Sequoia imaging device equipped with a $3.5-\mathrm{MHz}$ ultrasound transducer (Acuson Corporation, Mountain View, CA) preoperatively, at discharge and at follow-up appointments. Echocardiographic measurements were averaged over three cardiac cycles. Inter-observer (I.C e C.R) and intra-observer variability for measurement of DT and end systolic volume (ESV) were $<5 \%$ (range for intra-observer 0.7 to $3.1 \%$, inter-observer 0.6 to $4.6 \%$ ). 
LV volume and left ventricular ejection fraction (LVEF) were assessed by the bi-apical Simpson disk method ${ }^{10}$. The sphericity index (SI) was obtained at end diastole and end systole ( $\left(\mathrm{SI}_{\mathrm{D}}\right.$ and $S \mathrm{I}_{S}$, respectively) as the volume of the left ventricle divided by the volume of a sphere with a diameter equal to the longest axis of the left ventricle measured in the apical view ${ }^{11}$. The wall motion score index (WMSI) was calculated according to a 17 -segment model ${ }^{12}$. According to Stellbrink et cowor$\operatorname{kers}^{13}$ a decrease in LV end systolic volume $>15 \%$ from baseline value was considered LVRR.

\section{Quantification of MR}

The following quantitative measurements were simultaneously employed to grade the severity of MR and final results were averages of measured values ${ }^{14-15}: 1$ ) Quantitative Doppler. (2) Proximal Isovelocity Surface Area (PISA). These methods were averaged, allowing calculation of regurgitant volume (RV), regurgitant fraction (RF) and effective regurgitant orifice (ERO). In patients with no or trivial MR by color Doppler, RV and RF were used as calculated, and ERO was assumed as null.

Recurrent MR was the insufficiency $\geq 2+$ at follow up appointments in patients with no/trivial MR at discharge.

\section{Diastolic Filling Patterns}

Diastole was evaluated by measuring transmitral flow. Peak early transmitral flow velocity (E), peak late transmitral flow velocity (A), E/A ratio and deceleration time of $E$ velocity (DT) were obtained by pulsed Doppler ${ }^{16}$. The isovolumic relaxation time (IVRT) was obtained by continuous wave Doppler with the wave beam directed from the apical five-chamber view across the region between the aortic outflow tract and the inflow tract.

Finally, systolic and diastolic pulmonary venous flow and their ratio (S/D) were routinely assessed ${ }^{16}$.

\section{Patient Classification}

According to baseline $S / D$ and $D T$, irrespective of filling profile, patients were assigned to one of four Groups ${ }^{16}$ : Group $1(n=48)$, normal pattern $(S / D \geq 1$; DT $<220 \mathrm{msec}$ ); Group 2 ( $\mathrm{n}=61)$, impaired relaxation LV filling pattern $(\mathrm{S} / \mathrm{D} \geq 1 ; \mathrm{DT}>$ $220 \mathrm{msec}$ ); Group 3 ( $\mathrm{n}=60)$, pseudonormal LV filling pattern ( $/ \mathrm{D}<1$; DT $140-200$ msec); Group $4(\mathrm{n}=65)$, restrictive LV filling pattern (S/D $<1$; DT $<140 \mathrm{msec})$.The 140 value was chosen because it unequivocally identifies patients with restrictive filling 
and worse prognosis after myocardial infarction ${ }^{17}$. In restrictive and pseudonormal patients with E/A between 1 and 2, the Valsalva maneuver was performed to unmask the presence of impaired relaxation ${ }^{18}$. As reported in Table 1, preoperative demographic, clinical and intraoperative data were comparable in the four groups. Table 2 shows baseline echocardiographic findings: patients in the Group 4 had larger LV diameters and volumes and showed a more spherical and dysfunctioning left ventricle.

\section{Statistical Analysis}

The sample size was determined by GraphPad StatMate release 2.00 (GraphPad Prism Software, Inc, San Diego, CA).on the basis of preliminary data obtained by echocardiography and was determined on the basis of the following assumptions: Type I error of 0.05 (two-sided), power of $80 \%$, difference in end systolic volume between patients with or without recurrent mitral regurgitation of 0.78 , standard deviation of 2.4. The calculated study population was 300 . However 270 patients were recruited to allow for possible analytic problems while processing the eventualities potentially leading to patient attrition. All data were analyzed with the SPSS for Windows, release 15.0 (SPSS, Inc, Chicago, IL) statistical package and with GraphPad Prism for Windows, release 4.0 (Graph-Pad Prism Software, Inc, San Diego, CA). Variables were tested for normal distribution by the Kolmogorov-Smirnov test. Continuous data were expressed as mean $\pm \mathrm{SD}$ and compared using repeated ANOVA with the Tukey post-hoc test for multiple groups comparison. Comparison of proportions was performed using contingency $\chi^{2}$ analyses or Fisher's test, where appropriate. Non-normally distributed variables were presented as median and interquartile range. The Mann-Whitney and Kruskall-Wallis tests were used to compare non-parametric variables with Dunn's test for multiple comparisons. Cox Regression analysis was employed to identify multivariable predictors of late mortality. Multivariable logistic regression analysis, by means of a backward stepwise algorithm (cut-off for entry 0.05 , for removal 0.10 ) was performed to identify predictors of early mortality and recurrence of MR. Forty-nine demographic, clinical and Doppler echocardiographic variables were investigated for their predictive value of LVRR. Categorical variables with more than two levels in the regression model were converted into dummy variables. To enhance the accuracy of the model, the number of variables were reduced using variable clustering ${ }^{19,20}$. Model assumptions (linearity and additivity assumptions) were checked by piecewise cubic polynomials (spiline functions) and pooled interaction test ${ }^{21}$, respectively, and found to be satisfied. Goodness of fit of the final logistic regression models was assessed with the Hosmer-Lemeshow statistic ${ }^{20}$ and predictive accuracy was assessed by the concordance (c) index ${ }^{21}$. Internal validation of predictors generated by multivariable 


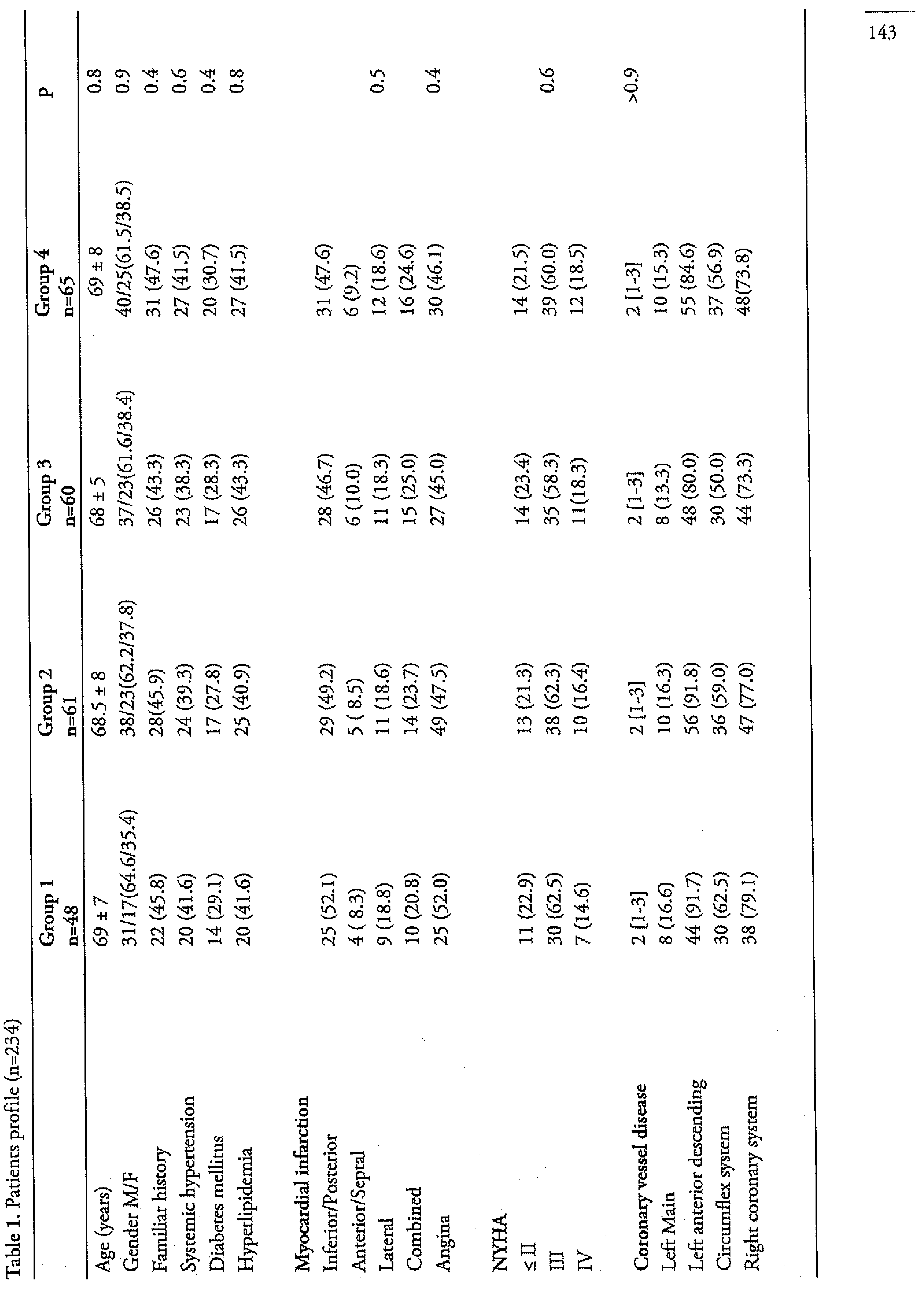




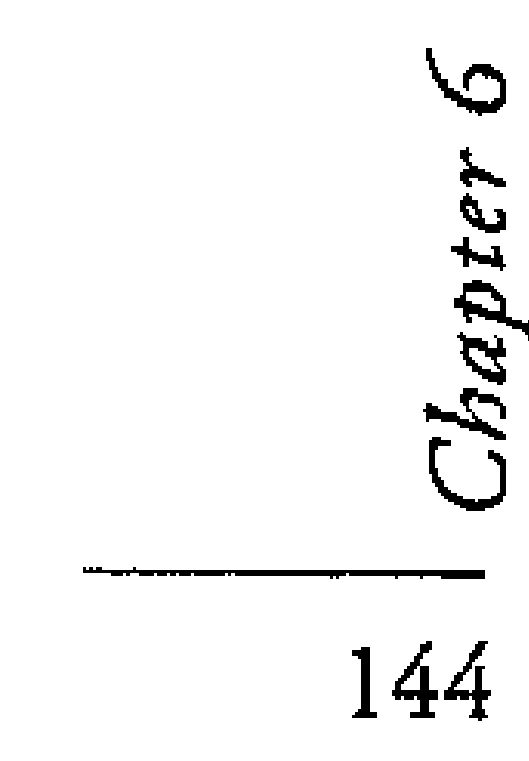

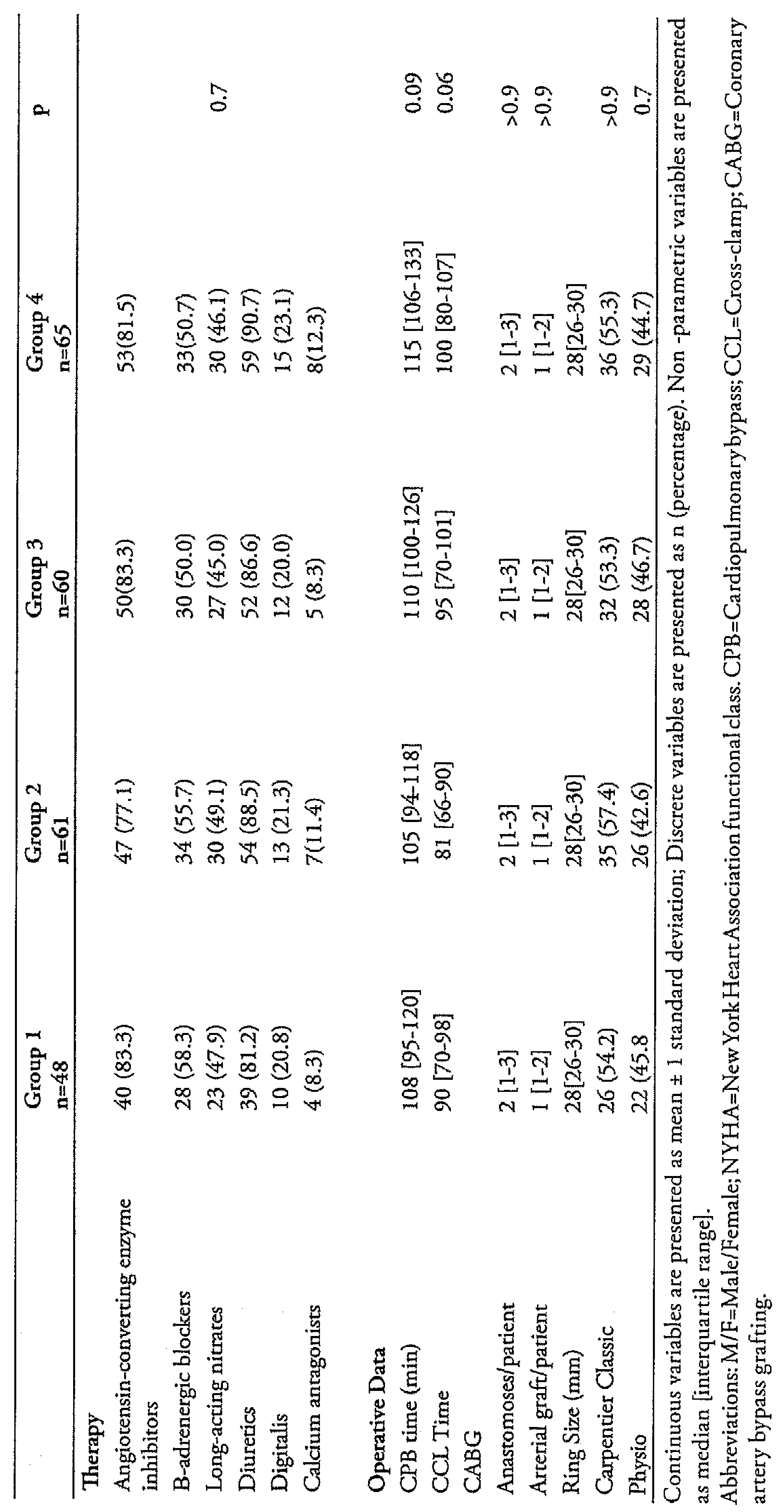


Table 2. Baseline echocardiography

\begin{tabular}{llllll} 
& $\begin{array}{l}\text { Group 1 } \\
\mathrm{n}=48\end{array}$ & $\begin{array}{l}\text { Group 2 } \\
\mathrm{n}=61\end{array}$ & $\begin{array}{l}\text { Group 3 } \\
\mathrm{n}=60\end{array}$ & $\begin{array}{l}\text { Group 4 } \\
\mathrm{n}=65\end{array}$ & $\mathrm{p}$ \\
\hline MR Grade & $3[3-4]$ & $3[3-4]$ & $3[3-4]$ & $3[3-4]$ & $>0.9$
\end{tabular}

MR Quantitative data

$\begin{array}{lccccc}\text { TA } & 3.5 \pm 1.1 & 3.7 \pm 1.0 & 3.2 \pm 1.1 & 3.5 \pm 1.1 & 0.6 \\ \text { ERO } & 37 \pm 12 & 36 \pm 11 & 38 \pm 14 & 40 \pm 16 & 0.42 \\ \mathrm{RF} & 46 \pm 13 & 44 \pm 12 & 47 \pm 13 & 50 \pm 18 & 0.55 \\ \mathrm{RV} & 57 \pm 17 & 56 \pm 15 & 58 \pm 18 & 60 \pm 16 & 0.72 \\ \mathrm{CH} & 1.0 \pm 0.2 & 1.9 \pm 0.2 & 1.2 \pm 0.2 & 1.4 \pm 0.2 & 0.001 \\ & & & & & \\ \text { Left ventricular remodeling } & & & & & \\ \text { EDD } & 59 \pm 6 & 60 \pm 7 & 56 \pm 7 & 65 \pm 8 & 0.03 \\ \text { ESD } & 49 \pm 7 & 51 \pm 8 & 47 \pm 7 & 56 \pm 9 & 0.03 \\ \text { LA } & 49 \pm 6 & 50 \pm 7 & 48 \pm 3 & 47 \pm 7 & 0.7 \\ \text { ESV } & 109 \pm 22 & 110 \pm 24 & 112 \pm 31 & 140 \pm 26 & <0.001 \\ \text { EDV } & 176 \pm 37 & 165 \pm 35 & 176 \pm 28 & 194 \pm 30 & 0.04 \\ \text { LVEF } & 38 \pm 12 & 33 \pm 12 & 36 \pm 10 & 27 \pm 11 & 0.007 \\ \text { SI } & 0.56 \pm 0.1 & 0.55 \pm 0.2 & 0.52 \pm 0.1 & 0.75 \pm 0.1 & <0.001 \\ \text { SI } & 0.64 \pm 0.1 & 0.62 \pm 0.1 & 0.62 \pm 0.1 & 0.80 \pm 0.1 & <0.001 \\ \text { WMSI } & 1.10 \pm 0.5 & 1.14 \pm 0.7 & 1.15 \pm 0.6 & 1.71 \pm 0.7 & <0.001\end{array}$

\section{Diastolic function}

\begin{tabular}{lccccc} 
E/A ratio & $1.1 \pm 0.3$ & $0.6 \pm 0.08$ & $1.5 \pm 0.3$ & $1.9 \pm 0.6$ & 0.04 \\
DT & $215 \pm 25$ & $235 \pm 28$ & $200 \pm 21$ & $119 \pm 12$ & $<0.001$ \\
IVRT & $95 \pm 7$ & $112 \pm 9$ & $95 \pm 10$ & $54 \pm 10$ & 0.02 \\
S/D & $1.1 \pm 0.3$ & $1.0 \pm 0.2$ & $0.93 \pm 0.1$ & $0.7 \pm 0.1$ & $<0.001$ \\
\hline
\end{tabular}

Continuous variables are presented as mean \pm standard deviation;. Non-parametric variables were presented as median [Interquartile range].

Abbreviations: $M R=M i t r a l$ Regurgitation; TA=Tenting area $\left(\mathrm{cm}^{2}\right)$; $E R O=$ effective regurgitant orifice $\left(\mathrm{mm}^{2}\right) ; \mathrm{RF}=$ Regurgitant fraction (\%); RV=Regurgitant volume (ml/beat); $\mathrm{CH}=$ Coaptation height $(\mathrm{cm}) ; \mathrm{EDD}=($ Left ventricular) End diastolic diameter $(\mathrm{mm}) ; \mathrm{ESD}=($ Left ventricular) End systolic diameter $(\mathrm{mm}) ; \mathrm{LA}=\mathrm{Left}$ atrium $(\mathrm{mm}) ; \mathrm{ESV}=($ Left ventricular) End systolic volume $(\mathrm{ml}) ; \mathrm{EDV}=(\mathrm{Left}$ ventricular) End diastolic volume $(\mathrm{ml}) ; \mathrm{LVEF}=\mathrm{Left}$ ventricular ejection fraction (\%); SPI $=$ Systolic Sphericity Index ; $S_{D}=$ Diastolic Sphericity Index WMSI=Wall Motion Score Index; E=Peak trans-

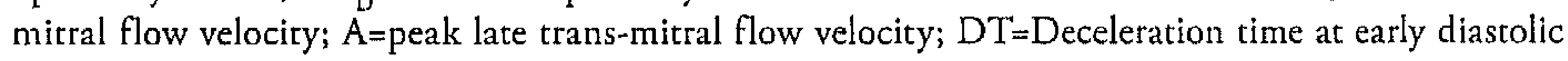
filling (ms); IVRT=Isovolumetric relaxation time (ms); S/D Systolic to diastolic pulmonary venous flow tatio. 
logistic regression was performed by means of bootstrapping techniques, with 1000 cycles and generation of $\mathrm{OR}$ and bias corrected $95 \% \mathrm{CI}^{21}$.

Optimal cutoff values were determined as the rounding cut-off that gives the maximum sum of sensitivity and specificity. This value should be the shoulder at the top left of the ROC (receiver operating characteristic curve). Bootstrapping techniques were employed to validate the results.

SPSS 12.0 (SPSS, Chicago, IL, USA) and Stats Direct 2.5.7 (Stats Direct, Sale, UK) were used for these calculations. Significance for hypothesis testing was set at the 0.05 two-tailed level.

\section{RESULTS}

\section{Clinical Assessment}

Early (30-day) mortality rates were $1.6 \%(n=1$, respiratory failure), $2.5 \%(n=1$, low-output syndrome) $3.3 \%(\mathrm{n}=2$, multiorgan failure, sepsis) and $9.2 \%(n=6$, four low-output syndrome, two multiorgan failure) in Groups 1,2,3 and 4, respectively $(p<0.001)$. Deceleration time was an independent predictor of early mortality at multivariable logistic regression (Table 3, $\mathrm{p}<0.001$ ). Other independent predictors were $\mathrm{SI}_{\mathrm{S}}(\mathrm{p}=0.001)$, WMSI $(\mathrm{p}=0.008)$ and $\mathrm{S} / \mathrm{D}(\mathrm{p}=0.004)$ whereas the other diastolic variables were not significant.

Internal validation of such multivariable analysis by means of bootstrapping, including the same variables of standard logistic regression model, further confirmed the significant predictive of early death (bootstrap OR $=2.6,95 \%$ bias-corrected $95 \%$ $\mathrm{CI}=1.5-6.0$ ). Finally, logistic regression model was reliable (Hosmer-Lemeshow test, $\mathrm{p}=0.7$ ) and accurate ( $\mathrm{c}$ index $=0.8$ ).

ROC analysis showed that DT had $100 \%$ of sensitivity and specificity with an optimal cutoff $<140 \mathrm{~ms}(95 \% \mathrm{CI}$ by bootstrapping $115-164 \mathrm{~ms})$ [area under curve $1 ; 95 \% \mathrm{CI} 0.70-1 ; \mathrm{p}<0.001]$. $\mathrm{SI}_{\mathrm{s}}$ with a cutoff $\geq 0.7$ ( $95 \% \mathrm{CI}$ by bootstrapping $0.58-$ 0.81 ) had $85 \%$ of sensitivity and $84 \%$ of specificity (area under curve $0.94 ; 95 \%$ CI $0.67-1 ; \mathrm{p}<0.001)$. In addition, WMSI $\geq 1.5$ (95\% Cl by bootstrapping $1.35-1.62)$ had $80 \%$ of sensitivity and $82 \%$ of specificity (area under curve $0.81 ; 95 \%$ CI $0.77-0.91$; $\mathrm{p}=0.002)$. Finally, $\mathrm{S} / \mathrm{D}<0.8(95 \% \mathrm{CI}$ by bootstrapping $0.63-0.91)$ had $90 \%$ of sensitivity and specificity (area under curve $0.78 ; 95 \%$ CI 0.65-0.87; $\mathrm{p}=0.001$ ).

During the follow-up fifteen patients died: 1 in the normal Group (3 months, respiratory failure), 2 in the impaired relaxation Group (36 months, heart failure; 31 months, myocardial infarction) 3 in the pseudonormal Group ( 6 months, multiorgan failure; 8 months infection; 14 months respiratory failure) and 9 in the restrictive Group (stroke $\mathrm{n}=2$ [12 and 22 months]; heart failure $\mathrm{n}=2$ [ 45 and 54 months]; 
Table 3. Predictors of early and late death

\begin{tabular}{|c|c|c|c|c|}
\hline \multicolumn{3}{|l|}{ Early } & \multicolumn{2}{|c|}{$95 \%$ Bias corrected CI } \\
\hline Variable & Odds Ratio & $\mathrm{p}$ & Lower Limit & Upper Limit \\
\hline DT & 3.05 & $<0.001$ & 1.5 & 8.1 \\
\hline $\mathrm{SI}_{\mathrm{s}}$ & 2.40 & 0.001 & 1.4 & 3.9 \\
\hline WMSI & 1.02 & 0.008 & 1.0 & 2.0 \\
\hline$S / D$ & 1.22 & 0.04 & 0.8 & 2.1 \\
\hline \multicolumn{3}{|l|}{ Late } & \multicolumn{2}{|c|}{$95 \%$ Bias corrected CI } \\
\hline Variable & Hazard Ratio & $\mathrm{p}$ & Lower Limit & Upper Limit \\
\hline $\mathrm{DT}<140 \mathrm{~ms}$ & 2.9 & $<0.001$ & 1.6 & 4.2 \\
\hline$S / D<0.8$ & 2.4 & $<0.001$ & 1.5 & 3.8 \\
\hline$S I_{s} \geq 0.7$ & 1.9 & 0.001 & 1.1 & 3.3 \\
\hline WMSI $\geq 1.5$ & 1.6 & 0.003 & 1.1 & 2.2 \\
\hline
\end{tabular}

Abbreviations: $L V=L$ eft Ventricle; $D T=$ Deceleration time $S I_{S}=$ Systolic Sphericity Index ;

WMSI=Wall Motion Score Index; S/D Systolic to diastolic pulmonary venous flow ratio.

malignancy $\mathrm{n}=2$ [55 and 59 months]; myocardial infarction, 42 months; arrhythmia, 59 months; sudden unexplained death, 22 months).

Six-year actuarial survival was $95.2 \pm 3.8 \%, 93.3 \pm 4.6 \%, 89.8 \pm 10.7 \%$ and 64.2 $\pm 6.6 \%$ in Groups $1,2,3$ and 4, respectively ( $p=0.022$, Figure 1$)$. A Cox regression analysis revealed that DT $<140 \mathrm{~ms}(\mathrm{p}<0.001)$ and $S / \mathrm{D}<0.8(\mathrm{p}<0.001)$ were independent predictor of late mortality (Table 3). Other predictors were $S I \geq 0.7 \quad(p=0.001)$ and WMSI $\geq 1.5$ ( $\mathrm{p}=0.003)$. Other diastolic filling patterns were not significant.

At late control, mean NYHA class was $2.1 \pm 0.3,2.3 \pm 0.4,2.2 \pm 0.4$ and $3 \pm$ 0.6 in Groups 1, 2, 3 and 4, respectively $(p<0.001) .70 .5 \%$ patients in the Group 4 had NYHA $\geq 3 \%$ vs. $33.3 \%$ in the pseudonormal group $(p<0.001), 18.1 \%$ in the impaired relaxation group $(p<0.001)$ and $4.6 \%$ in the normal Group ( $<<0.001)$.

\section{Postoperative diastolic function}

Indices of diastolic function (Figure 2A-D) remained stable over time in patients with baseline diastolic filling. In patients with impaired relaxation, E/A increased $(p=0.04)$, whereas DT $(p=0.03)$ and IVRT $(p=0.02)$ reduced at discharge without further changes at subsequent controls. S/D did not show significant changes in this Group.

In patients with pseudonormal filling, $E / A$ reduced $(\mathrm{p}=0.001)$ whereas DT $(p=0.03)$, IVRT $(p<0.001)$ and $S / D(p=0.03)$ increased ad discharge. All these values remained constant afterwards. Index of diastolic function did not modify over time in patients with restrictive filling pattern. 


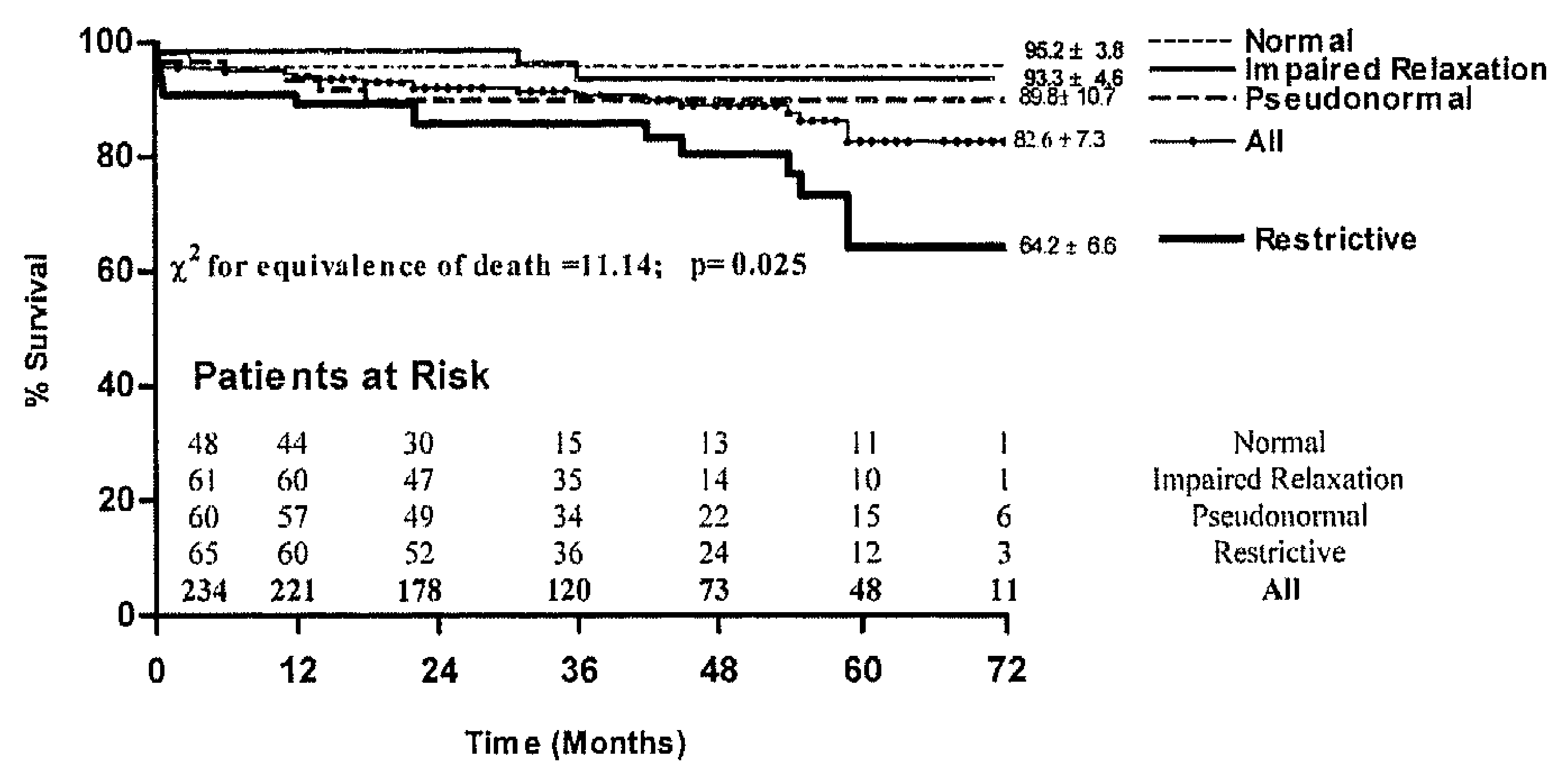

Figure 1. Six-year actuarial survival by group (see text).

\section{Diastolic Function}

E/A

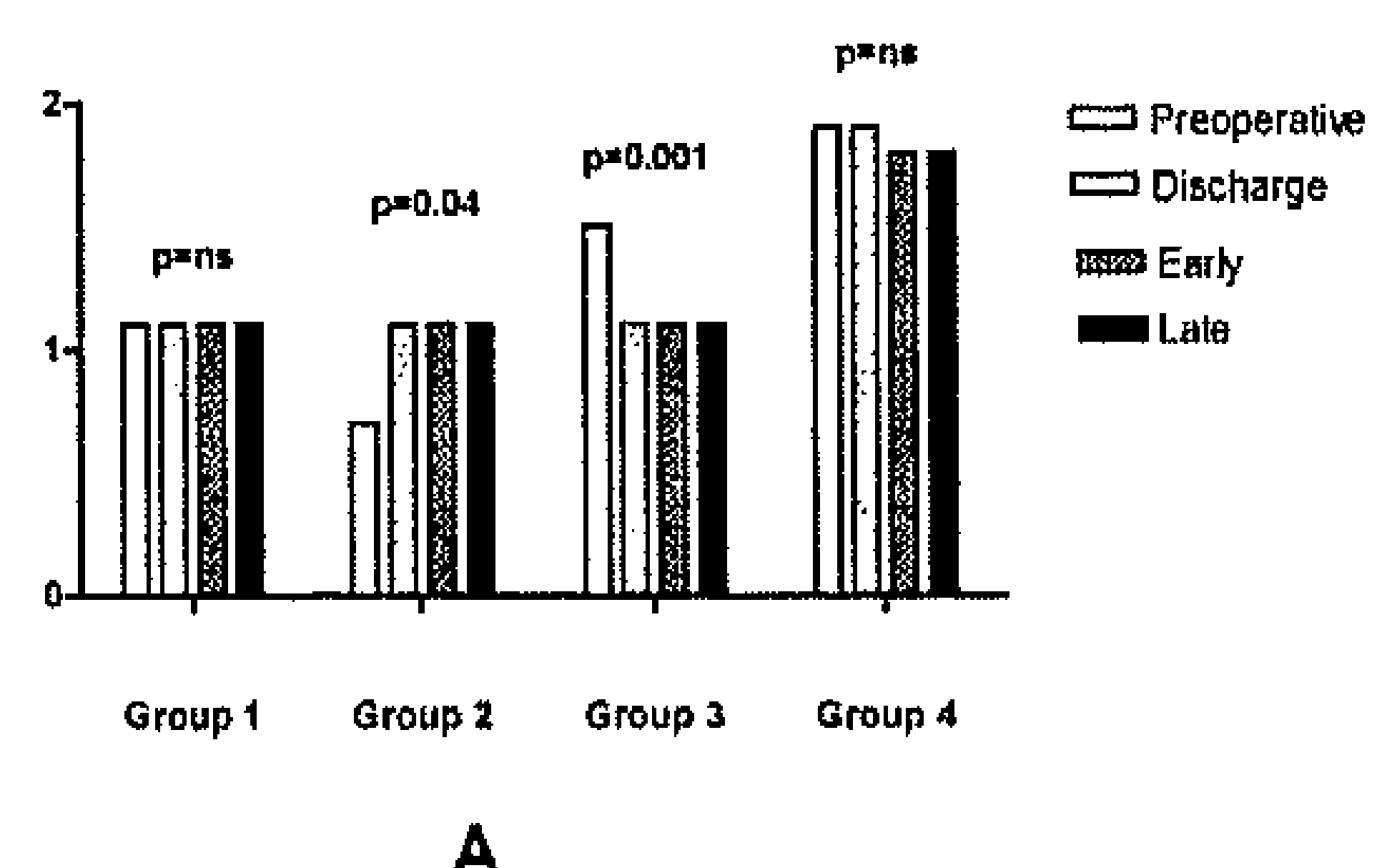

IVRT

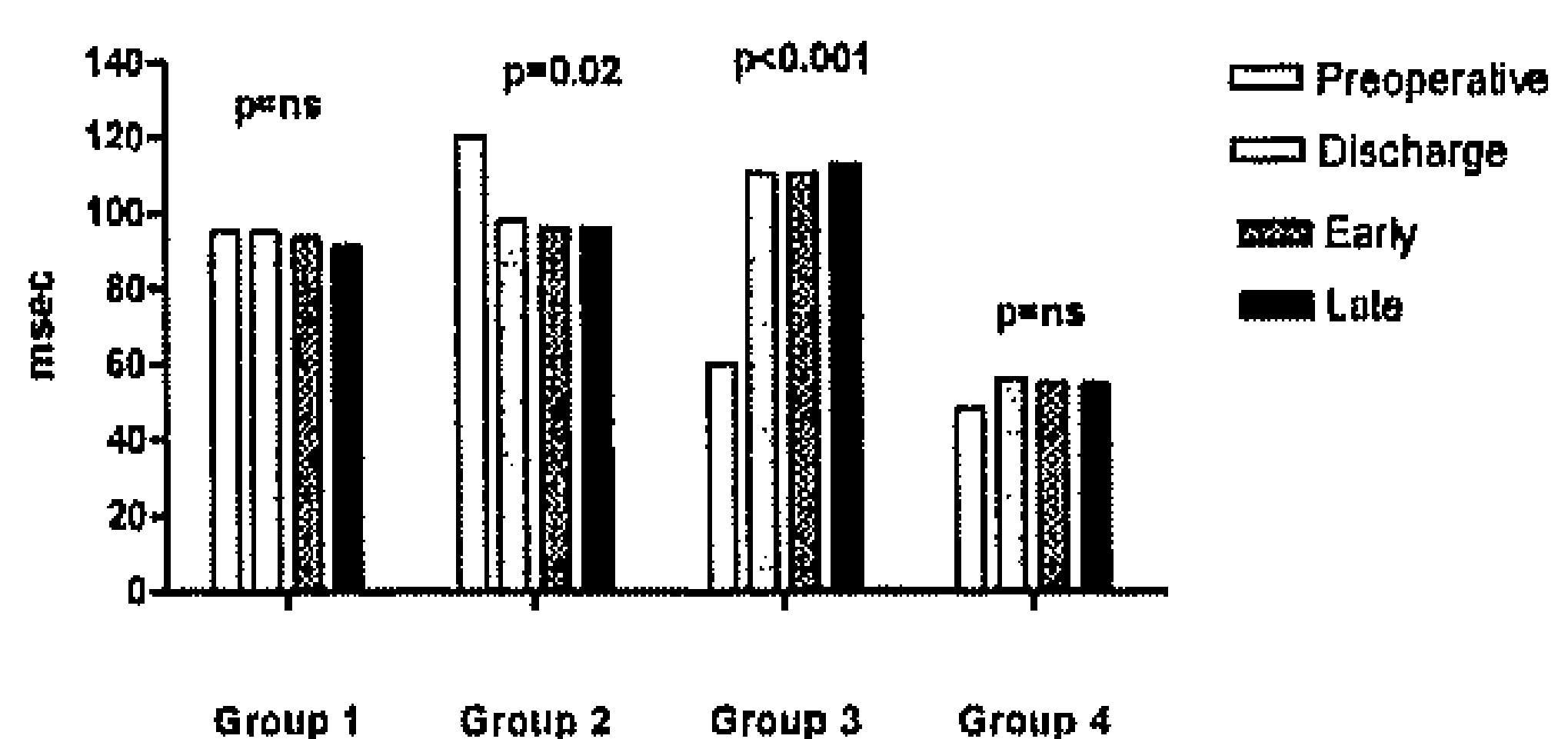

C

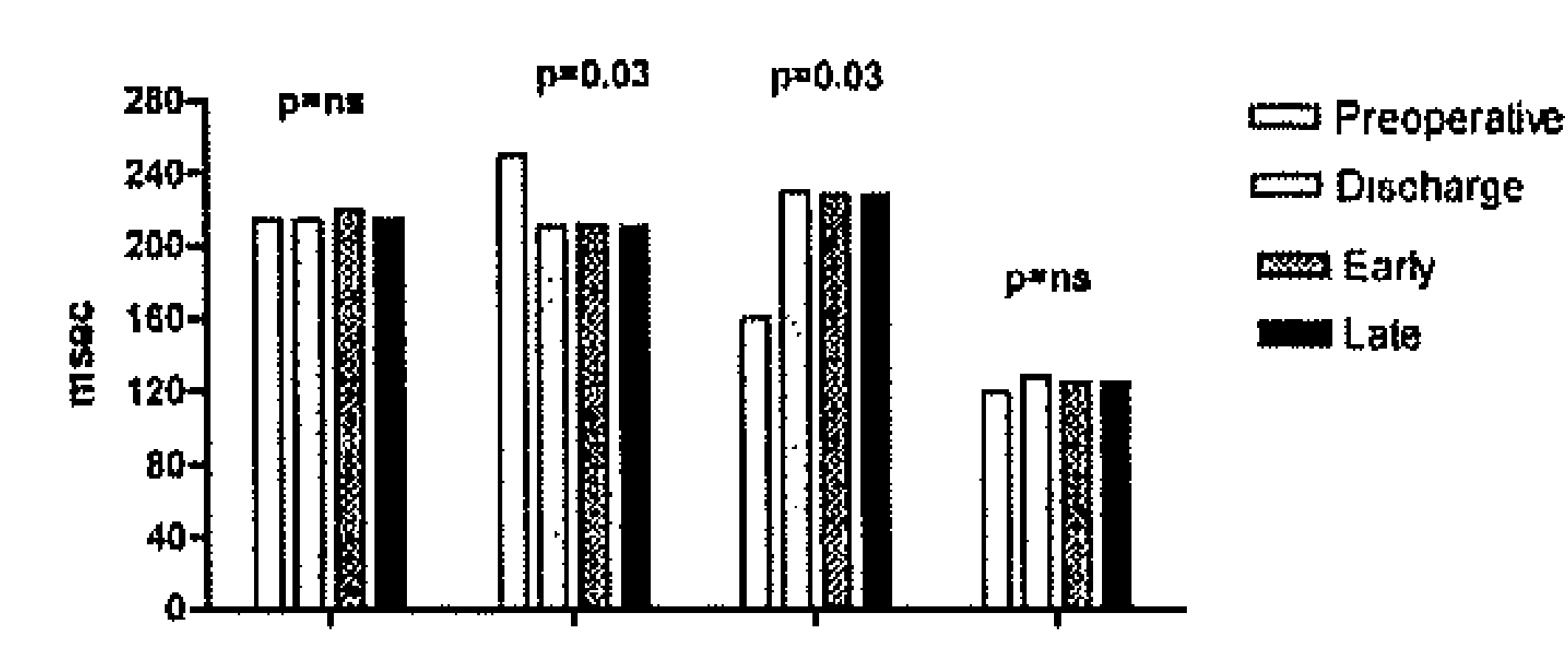

Group 1 Group 2 Group 3 Group 4

B

$S / D$

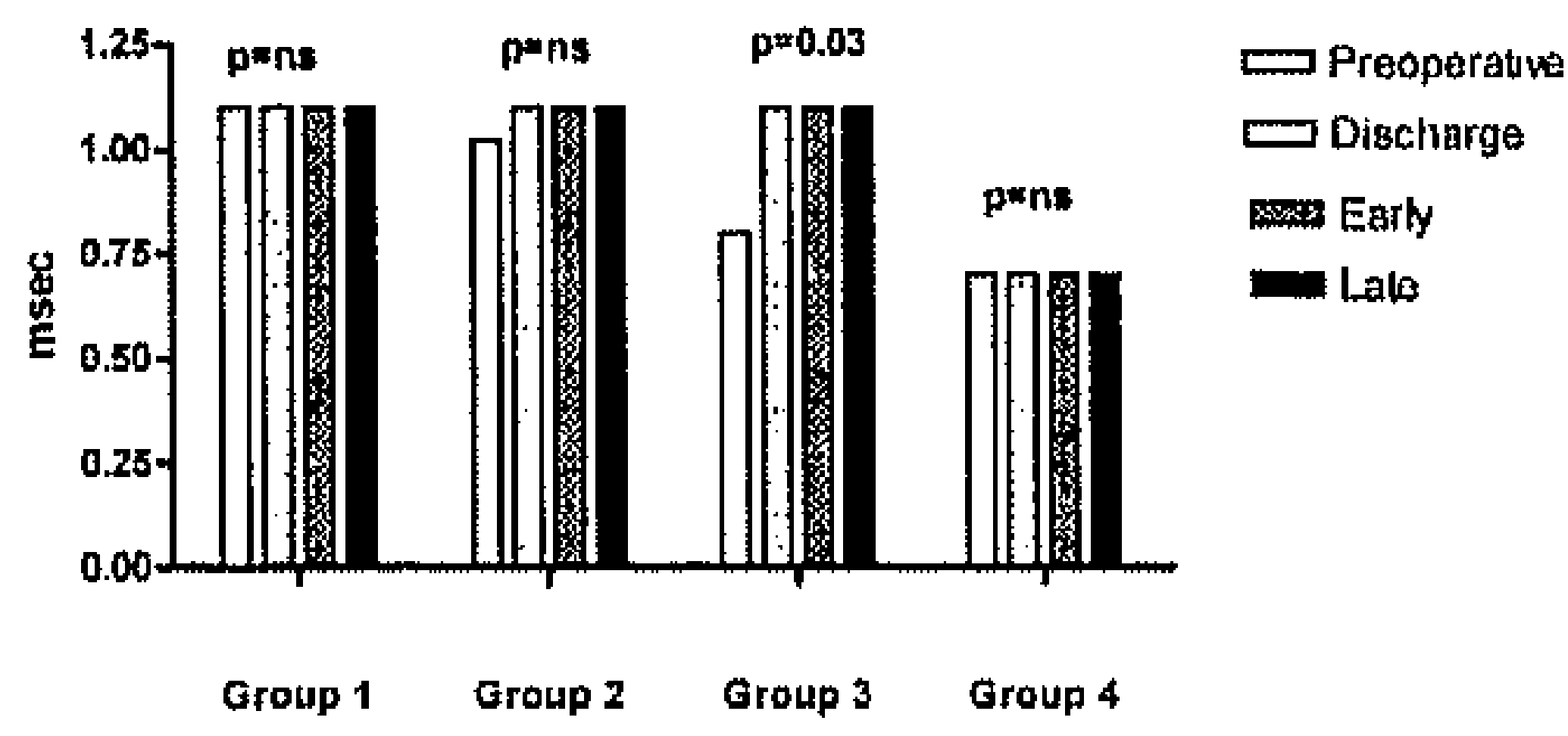

D

Figure 2. A-D.Diastolic Function by Group (see text). Abbreviations: $\mathrm{E} / \mathrm{A}=$ Peak trans-mitral flow velocity/ peak late trans-mitral flow velocity ratio; $\mathrm{DT}=$ Deceleration time at early diastolic filling; IVRT= Isovolumetric relaxation time; $S / D=$ Systolic to diastolic pulmonary venous flow ratio. 
Mitral regurgitation was significantly reduced at discharge in all groups $(p<0.001)$ and it did not change at following controls in Groups 1, 2 and 3 (Table 3). In contrast, in patients with restrictive filling pattern, it remained stable at early control, whereas it increased at further controls $(\mathrm{p}<.001)$. Indeed, at late follow-up, among patients in group $4,58.4 \%(n=38)$ had an $M R$ grade $\geq 2$ vs. $8.3 \%(n=4), 9.8 \%$ $(\mathrm{n}=6)$ and $8.3 \%(\mathrm{n}=5)$ in Groups 1, 2, and 3, respectively $(\mathrm{p}<0.001)$.

Multivariable logistic regression analysis identified systolic sphericity Index $(\mathrm{OR}=4.97,95 \%$ bias corrected $\mathrm{CI}=2.11-6.40, \mathrm{p}<0.001)$, $\mathrm{DT}(\mathrm{OR}=4.66,95 \% \mathrm{CI}=$ 2.01-5.43, $\mathrm{p}<0.001)$, end systolic volume $(\mathrm{OR}=2.59,95 \%$ bias corrected $\mathrm{CI}=1.19$ 3.77, $\mathrm{p}=0.003)$ and wall motion score Index $(\mathrm{OR}=1.99,95 \% \mathrm{CI}=0.88-2.37, \mathrm{p}=0.04)$ as independent predictors of recurrent MR.

Internal validation of such multivariable analysis by means of bootsrapping, including the same variables of standard logistic regression model, further confirmed the significant predictive of recurrent MR (bootstrap $\mathrm{OR}=2.3,95 \%$ bias-corrected $95 \% \mathrm{CI}=1.1-5.2$ ). Finally, logistic regression model was reliable (Hosmer-Lemeshow test, $\mathrm{p}=0.8$ ) and accurate ( $\mathrm{c}$ index $=0.78$ ).

ROC analysis showed that $\mathrm{SI}_{S}$ had $100 \%$ of sensitivity and specificity with an optimal cutoff $\geq 0.7$ ( $95 \%$ CI by bootstrapping $0.58-0.81$ ) [area under curve $1 ; 95 \%$ CI $0.70-1 ; \mathrm{p}<0.001)$. DT with a cutoff of $<142(95 \% \mathrm{CI}$ by bootstrapping $134-150)$ had $87 \%$ of sensitivity and $80 \%$ of specificity (area under curve $0.94 ; 95 \% \mathrm{Cl} 0.67-1$; $\mathrm{p}<0.001$ ). In addition, ESV $\geq 145 \mathrm{ml}$ (95\% CI by bootstrapping 134-155) had $90 \%$ of sensitivity and specificity (area under curve $0.87 ; 95 \%$ CI $0.80-0.94 ; \mathrm{p}<0.001$ ). Finally, WMSI $\geq 1.5$ ( $95 \%$ CI by bootstrapping $1.35-1.62$ ) had $80 \%$ of sensitivity and $82 \%$ of specificity (area under curve $0.81 ; 95 \% \mathrm{CI} 0.77-0.91 ; \mathrm{p}=0.002$ ).

\section{Echocardiographic Results}

Table 4 shows postoperative echocardiography. In normal, impaired relaxation and pseudonormal groups, ESV decreased significantly at discharge $(\mathrm{p}<0.001)$, early $(p<0.001)$ and late controls $(p<0.001)$. In contrast, in the restrictive group, ESV was significantly reduced at discharge $(p=0.01)$ and early follow up $(p=0.02)$ but increased again $(\mathrm{p}<0.001)$ at late control. A $15 \%$ reduction in ESV, considered indicative of reverse remodeling ${ }^{13}$ occurred in $95.7 \%, 96.3 \%, 88.3 \%$ and $0 \%$ in Groups 1,2, 3 , and 4, respectively $(\mathrm{p}<0.001)$. Changes in EDV, ESD, EDD and WMSI mirrored those of ESV. Systolic and diastolic sphericity indexes improved in Groups 1, 2, and 3 at discharge $\left(p<0.001\right.$ ), early (Group $1, p=.01$ for both $\mathrm{SI}_{S}$ and $\mathrm{SI}_{\mathrm{D}}$; Group 2, $\mathrm{p}<0.001$ for $\mathrm{SI}_{\mathrm{S}}$ and $\mathrm{p}=0.02$ for $\mathrm{SI}_{\mathrm{D}}$; Group 3, $\mathrm{p}=0.04$ for both $S I_{S}$ and $\mathrm{SI}_{\mathrm{D}}$ ) as well as at late control (Group 1, $\mathrm{p}=0.03$ for $\mathrm{SI}_{\mathrm{S}}$ and $\mathrm{p}=0.01$ for $\mathrm{SI}_{\mathrm{D}}$; Group 2, $\mathrm{p}=0.02$ for $\mathrm{SI}_{\mathrm{s}}$ and $\mathrm{p}=0.01$ for $\mathrm{SI}_{\mathrm{D}}$; Group 3, $\mathrm{p}<0.001$ for both $\mathrm{SI}_{S}$ and $S \mathrm{~S}_{\mathrm{D}}$ ). In Group 4 there was an initial, but not significant, reduction in both the systolic and diastolic sphericity 
$\frac{6}{150}$

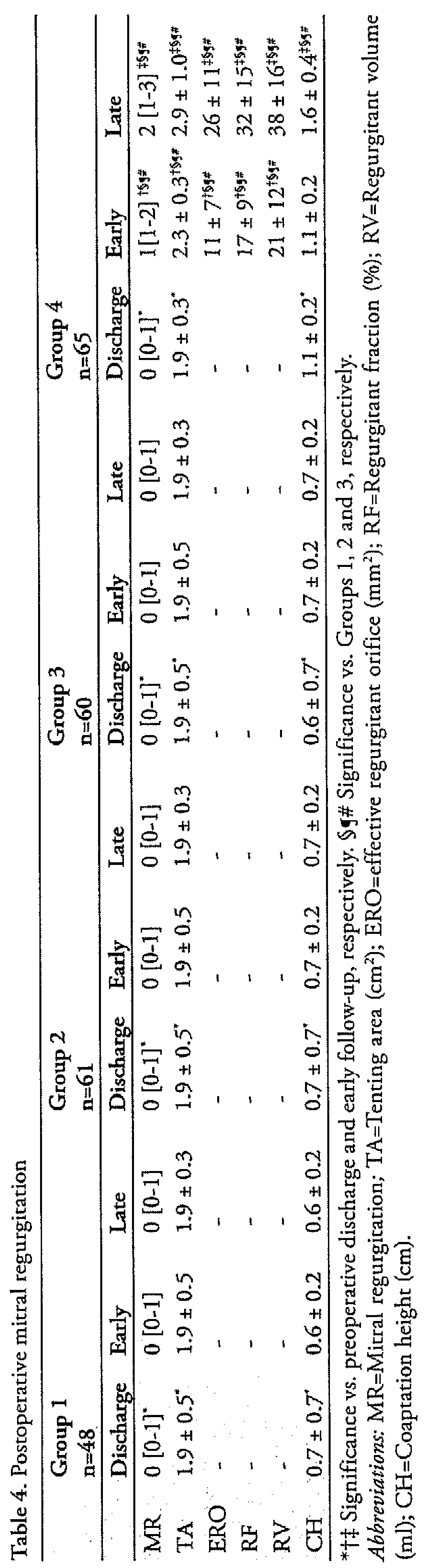




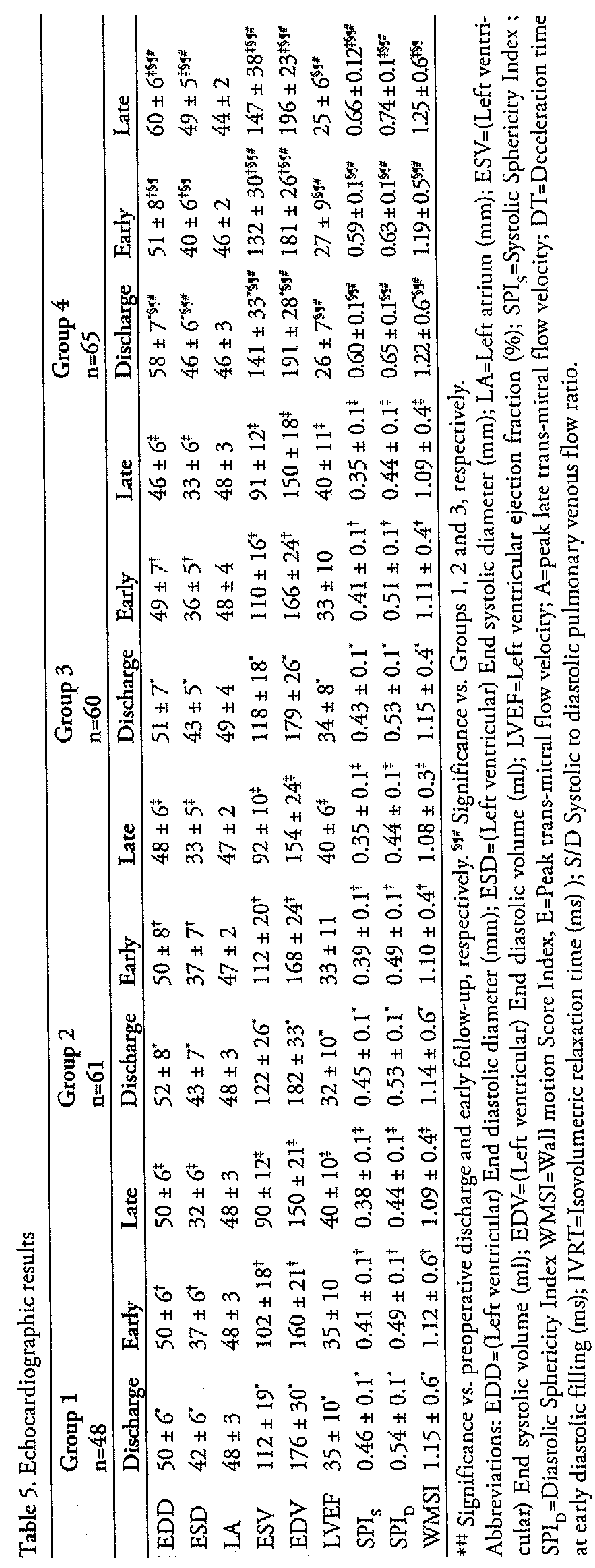


52 indexes, which remained stable at early follow-up with a late increase exceeding the preoperative value $(\mathrm{p}<0.001)$. In normal, impaired relaxation, and pseudonormal groups, LVEF significantly improved at discharge and at late follow-up, remaining unchanged at early control. In contrast, in the restrictive group it did not significantly change at any study point.

\section{DISCUSSION}

This study was aimed at exploring the predictive value of preoperative impairment of diastolic function on early and late outcome and recurrence of mitral regurgitation in patients with Chronic ischemic mitral regurgitation (CIMR) undergoing combined undersized mitral ring annuloplasty (UMRA) and coronary artery bypass grafting (CABG). For this purpose, patients of our cohort were divided into four Groups (normal diastolic pattern [ $\mathrm{n}=48]$, impaired relaxation [n=61], pseudonormal $[n=60]$, and restrictive $[n=65]$ ) according to baseline $S / D$ and DT, irrespective of the filling profile. The study protocol included early and late clinical assessment, recurrent mitral regurgitation and left ventricular reverse remodeling assessed by echocardiography preoperatively, at discharge and at follow-up appointments.

The early (30-day) mortality rate was higher in the restrictive group $(9.2 \%$, $p<0.001$ vs. Groups 1,2 and 3). In addition, Deceleration time $<140 \mathrm{~ms}$ and S/D $<0.8$ were strong predictors of early mortality at multivariable logistic regression $(\mathrm{p}<0.001$ and $\mathrm{p}=0.004$, respectively) whereas the other diastolic variables were not significant.. Other independent predictors of late death were $\mathrm{SI}_{\mathrm{S}}(\mathrm{p}=0.001)$ and WMSI $(p=0.008)$

Furthermore, 6-year actuarial survival was significantly lower in Group 4 (log-rank $=0.02$ ) and Cox regression analysis identified $D T<140 \mathrm{~ms}$ and $S / D<0.8$ $(\mathrm{p}<0.001)$ as independent predictors of late mortality. Other predictors were SI $\geq 0.7(p=0.001)$ and WMSI $\geq 1.5(p=0.003)$. Other diastolic filling patterns were not significant. In addition, at late control, $70.5 \%$ patients in the Group 4 had NYHA $\geq 3 \%$ ( $\mathrm{p}<0.001$ vs. Groups $1-3$ ).

Mitral regurgitation was significantly reduced at discharge in all groups $(\mathrm{p}<0.001)$ and it did not change at following controls in Groups 1, 2 and 3 whereas, in patients with restrictive filling pattern, it increased afterwards $(p<0.001)$. However, at late follow-up, among patients in group 4, 58.4\% ( $\mathrm{n}=38)$ had a MR grade $\geq 3$. Multivariable logistic regression analysis identified DT $(\mathrm{p}<0.001)$, systolic sphericity index $(\mathrm{p}<0.001)$, end systolic volume $(\mathrm{p}=0.003)$ and wall motion score Index $(\mathrm{p}=0.04)$ as independent predictors of recurrence MR

Ereminiene et $\mathrm{a}^{22}$ demonstrated that diastolic restrictive filling pattern and deceleration time were independent predictors of death and residual moderate MR, late (12 months) after ischemic mitral valve repair in 55 patients. Nonetheless these 
authors defined the restrictive filling pattern as an E/A ratio $>2$ or an E/A ratio 1-2 with DT $<150 \mathrm{msec}$ and IVRT $<60 \mathrm{~ms}$. E and E/A ratio are partially dependent on age, heart rate, loading conditions and degree of MR. In particular, mitral regurgitation may increase the E-wave velocity of transmitral flow, which resembles pseudonormal or restrictive patterns. Several studies have shown that a short DT, even not load independent, irrespective of the ratio of early and late filling velocity $(\mathrm{E} / \mathrm{A})$ ratio, is strongly related to pulmonary capillary wedge pressure ${ }^{23,24}$ and end-diastolic pressure ${ }^{25}$, and it is an useful parameter for the serial assessment of LV diastolic function in post infarct patients also in those with $\mathrm{MR}^{26}$. For this reason, we used DT as a means of assessing LV filling, irrespective of filling pattern. Nonetheless, in an attempt to overcome the limitations of transmitral flow Doppler indices, we used routinely the assessment of pulmonary venous flow. Furthermore, we employed the Valsalva maneuver to unmask the presence of impaired relaxation in pseudonormal and restrictive Groups.

Interestingly, LVEF did not provide any prognostic value thus indicating that LV diastolic dysfunction may be more sensitive than LV systolic dysfunction in predicting death and recurrence of $M R$ after $C A B G$ and restrictive annuloplasty.

By comparing the four groups in our study, it appears evident that patients with preoperative restrictive filling pattern were much more globally remodeled at every study point; at late control they still showed a more spherical and enlarged LV. Particularly, the late increase in sphericity indexes in this group, which exceeded the preoperative value, was consistent with further remodeling after UMRA and reflects increased tethering on the mitral valve, thus translating into a significantly higher number of patients with late MR.

Finally, considering a $15 \%$ reduction in ESV indicative of reverse remodeling, it occurred in $95.7 \%, 96.3 \%, 88.3 \%$ and $0 \%$ in Groups $1,2,3$, and 4 , respectively $(\mathrm{p}<0.001)$.

\section{STUdY LIMITATIONS}

Our study findings should be viewed in light of some inherent limitations.

-Similarly to other studies ${ }^{22}$ we employed conventional indices of LV diastolic function.

Tissue Doppler imaging (TDI) would have been more useful in estimating LV diastolic filling pattern. Nonetheless, DT, even not load independent is strongly related to pulmonary capillary wedge pressure and end-diastolic pressure and it is an useful parameter for the serial assessment of LV diastolic function in post infarct patients, also in those with MR. Furthermore, in an attempt to overcome the limitations of transmitral flow Doppler indices, we used routinely the assessment of pulmonary venous flow. 
An evaluation of diastolic function after UMRA and CABG, employing TDI will be ongoing to confirm these findings.

Evaluation of LVRR was based on volumes obtained by echocardiography; Volumetry by 2D echocardiography depends on geometric assumptions and is subject to image plane positioning errors. Hence it is not accurate in left ventricles that are distorted in shape such as after myocardial infarction. However, this limitation belongs to most of published papers regarding this pathology.

- Viability testing was not performed in these patients. Therefore lack of LVRR in non responders might be also due to irreversible ischemic myocardial damage (not-viable myocardium). This issue deserves further investigation.

- Postoperative evaluation of the coronary status was not assessed. It would have been helpful to differentiate between surgical failure (valve repair and CABG) and the progress of the coronary disease.

- Fifteen patients with late deaths were not included in the study. They could have benefited from serial echocardiograms possibly with different results.

- Estimated cutoffs are known to be very susceptible to changes in the study population.

We employed bootstrapping techniques to validate the results, nonetheless, it has also been documented that the sensitivity/specificity associated with these cutoffs are overly optimistic.

\section{STREngth OF THE STUdY}

This is a large cohort reported with UMRA in CIMR patients with detailed (100\% complete) echocardiographic follow-up. Furthermore our patient cohort was more homogeneous than in other studies and does not display common clinical and methodological pitfalls that limit results from many studies of mitral valve repair ${ }^{27}$ : all patients underwent associated $C A B G$, they had no concomitant $M V$ procedures, and the entire cohort was uniform regarding the MV ischemic leaflet dysfunction. Moreover, to assess results for MV repair, we studied true "recurrent" MR excluding those patients with "residual" MR in whom the insufficiency was presumably never eliminated at surgery. Additionally, we undertook valve sizing in a standardized fashion and the degree of undersizing was homogeneous over the 5-year-period of the study. Finally, we used only two rings (Carpentier's rigid or semi-flexible rings).

\section{Clinical Implications.}

In our experience combined CABG and UMRA do not ensure successful and durable elimination of MR and significant LVRR in all patients. Those who can benefit from this approach could be preoperatively identified utilizing echo predictors. However, restrictive annuloplasty resulted to be ineffective in a large percentage 
of patients and results of this study suggest the need for different approaches directly addressing ventricular tethering in most of CIMR patients ${ }^{28}$. These procedures, as well as the employment of support devices ${ }^{29}$, were introduced in the recent years in clinical practice, thus our experience with these techniques is actually in the preliminary phase. Furthermore, mitral valve replacement, largely employed in the past in ischemic regurgitation, although eliminates the short-term risk of recurrent $M R$, it is associated with poor long-term survival ${ }^{30}$. Thus, in our actual policy, we do not consider MV replacement as a reasonable alternative to repair.

Another interesting finding of our study, apart from the predictive value of DT on Outcome and MR recurrence, was that in patients with more advanced diastolic dysfunction, evidenced by a LV restrictive filling, UMRA associated to CABG did not cause improvement of diastolic function. Contrastingly, an improvement of diastolic function occurred in impaired relaxation and Pseudonormal patients. This suggest that an early surgical indication is advisable in patients with CIMR in less severe stages of disease.In presence of advanced diastolic dysfunction, patients should be considered poor candidates for this procedure and concomitant or alternative procedure should be contemplated.

\section{CONCLUSIONS}

On the basis of our experience and with the above-mentioned limitations, we suggest that the assessment of potential candidates for UMRA should include accurate evaluation of LV diastolic function to identify patients suffering from CIMR who could really benefit from restrictive annuloplasty. Our findings need to be confirmed by further larger studies. 


\section{REFERENCES}

1. Grigioni F, Enriquez-Sarano M, Zehr KJ, Bailey KL, Tajik AJ. Ischemic Mitral Regurgitation. Long-term outcome and prognostic implications with quantitative Doppler assessment. Circulation 2001;103:1759-64.

2. Yiu SF, Enriquez-Sarano M, Tribouilloy C, Seward JB, Tajik AJ. Determinants of the degree of functional mitral regurgitation in parients with systolic left ventricular dysfunction: a quantitative clinical study. Circulation 2000;102:1400-6.

3. Lamas GA, Mitchell GF, Flaker GC, et al. Clinical significance of mitral regurgitation after acure myocardial infarction. Circulation 1997; 96:827-33

4. Kumanohoso T, Otsuji Y, Yoshifuku S. Mechanism of higher incidence of ischemic mitral regurgitation in patient with myocardial infarction: quantitative analysis of left ventricular and valve geometry in 103 patients with prior myocardial infarction. J THorac Cardiovasc Surg 2003;125:135-43.

5. Trichon BH, Felker GM, Shaw LK, Cabell CH, O'Connor CM. Relation of frequency and severity of mitral regurgitation to survival among patients with left ventricular systolic dysfunction and heart failure. Am J Card 2003;91:538-43.

6. Bach DS, Bolling SF. Improvement following correction of secondary mitral regurgitation in end-stage cardiomyopathy with mitral annuloplasty. Am J Cardiol. 1996;78:966-69.

7. Tibayan FA, Rodriguez F, Langer $\mathrm{F}$ et al. Undersized mitral annuloplasty alters left ventricular shape during acute ischemic mitral regurgitation. Circulation 2004;110[suppl.II]:II 98-II102.

8. Hausmann $H$, Siniawski $H$, Hetzer R. Mitral valve reconstruction and replacement for ischemic mitral insufficiency: Seven years follow-up. J Heart Valve Dis 1999;8:536-42.

9. Carpentier A. Cardiac valve surgery-The "French correction". J Thorac Cardiovasc. Surg. 1983; 86: 326-37.

10. Schiller NB, Shah PM, Crawford M et al..Recommendations for quantitation of the left ventricle by two-dimensional echocardiography. American Society of Echocardiography Committee on Standards, Subcommittee on Quantitation of Two-Dimensional Echocardiograms.J Am Soc Echocardiogr. 1989;2(5):358-67

11. Kono T, Sabbah HN, Rosman H, Alam M, Jafri S, Goldstein S. Left ventricular shape is primary determinant of functional mitral regurgitation in heart failure. J Am Coll Cardiol 1992;20:159498.

12. Cerqueira MD, Weissman NJ, Dilsizian V et al.American Heart Association Writing Group on Myocardial Segmentation and Registration for Cardiac Imaging. Standardized myocardial segmentation and nomenclature for tomographic imaging of the heart. A Statement for Healthcare Professionals From the Cardiac Imaging Committee of the Council on Clinical Cardiology of the American Heart Association. Circulation 2002;105: 539-42. 
13. Stellbrink C, Breithardt OA, Franke A, et al. PATH-CHF (Pacing Therapies in Congestive HeartFailure) Investigators; CPI Guidant Congestive Heart Failure Research Group. Impact of cardiac resynchronization therapy using hemodynamically optimizedpacing on left ventricular remodeling in patients with congestive heart failure andventricular conduction disturbances. J Am Coll Cardiol. 2001; 38(7):1957-65.

14. Gardin JM, Adams DB, Douglas PS et al. American Society of Echocardiography. Recommendations for a standardized report for adult transthoracic echocardiography: a report from the American Society of Echocardiography's Nomenclature and Standards Committee and Task Force for a Standardized Echocardiography Report.J Am Soc Echocardiogr 2002;15 (3):275-90.

15. Enriquez-Sarano M, Tribouilloy C. Quantitation of mitral regurgitation: rationale approach and interpretation in clinical practice. Heart 2002, 88 (suppl IV):1-4.

16. Garcia MJ, Thomas JD, Klein AL. New Doppler echocardiographic applications for the study of diastolic function. J Am Coll Cardiol 1998; 32:865-75.

17. Moller JE, Pellikka PA, Hillis GS, Oh JK.Prognostic importance of diastolic function and filling pressure in patients with acute myocardial infarction. Circulation. 2006 Aug 1;114(5):438-44.

18. Rakowski $\mathrm{H}$, Appleton $\mathrm{C}$, Chan $\mathrm{KL}$, et al. Canadian consensus recommendations for the measurement and reporting of diastolic dysfunction by echocardiography: from the Investigators of Consensus on Diastolic Dysfunction by Echocardiography. 1: J Am Soc Echocardingr. 1996;9(5):736-60. 28) Romesburg HC. Cluster analysis for researchers. North Carolina: Lulu Press;2004.1-330.

19. Harrel FE., Lee KL, Califf RM, Pryor DB, Rosati RA. Regression modeling strategies for improved prognostic prediction. Statistics in Medicine 1984;3:143-52.

20. Harrel FE Jr, Lee KL, Mark DB. Multivariable Prognostic Models: Issues in developing models, evaluating assumptions and adequacy, and measuring and reducing errors. Stats Med 1996:36187.

21. Hosmer DW, Lemeshow S. Applied Logistic regression.2nd ed.New York, NY: Wiley-Interscience; 2000:143-202.

22. Ereminiene E, Vaskelyte J, Benetis R, Stoskute N. Ischemic mitral valve repair: predictive significance of restrictive left ventricular diastolic filling. Echocardiography. 2005;22(3):217-24

23. Giannuzzi P, Imparato A, Temporelli PL, et al. Doppler-derived mitral deceleration time of early filling as a strong predictor of pulmonary capillary wedge pressure in postinfarction patients with left ventricular systolic dysfunction. J Am Coll Cardiol 1994;23:1630-7.

24. Appleton CP, Hatle LK, Popp RL. Relation of transmitral velocity patterns to

25. left ventricular diastolic function: new insights from a combined hemodynamic and Doppler echocardiographic study. J Am Coll Cardiol 1988;12:426-40.

26. Cerisano G, Bolognese L, Buonamici P, et al. Prognostic implications of restrictive left ventricular filling in reperfused anterior acute myocardial infarction. J Am Coll Cardiol 2001;37:793-9.

27. Braun J, Bax JJ, Versteegh MI et al. Preoperative left ventricular dimensions predict reverse remodeling following restrictive mitral annuloplasty in ischemic mitral regurgitation.Eur $\mathrm{J}$ Cardiothorac Surg 2005;27(5):847-53. 
28. Adams DH, Anyanwu AC. Pitfalls and limitations in measuring and interpreting the outcomes of mitral valve repair. J.Thorac Cardivasc Surg 2006; 131:523-9.

29. Borger MA, Alam A, Murphy PM, Doensr T, David TE. Chronic Ischemic Mitral Regurgitation: Repair Replace o Rethink? Ann Thorac Surg 2006; 81: 1153-61.

30. Konertz W, Rombeck B, Hotz H.Short-term safery of the Acorn cardiac support device in patients with advanced heart failure. J Am Coll Cardiol 2000;35:182A.

31. Gillinov AM, Wierup PN, Blackstone EH, et al.Is repair preferable to replacement for ischemic mitral regurgitation? J Thorac Cardiovasc Surg. 2001;122(6):1125-41. 


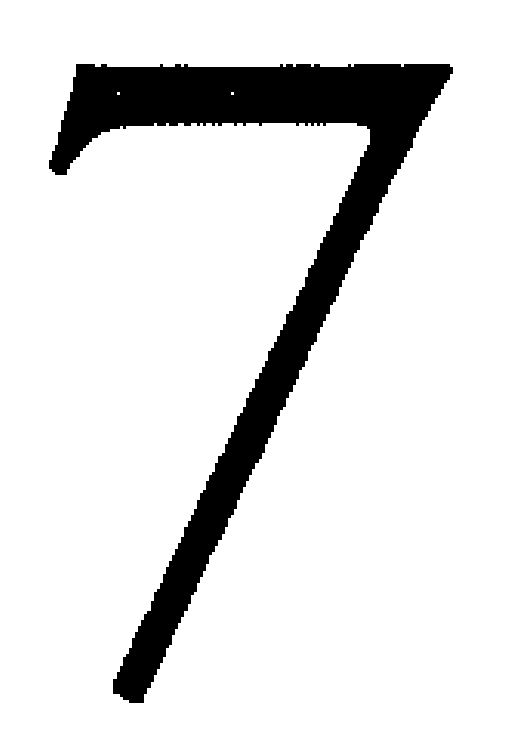

\section{Prognostic Value of Doppler-Derived Mitral Deceleration Time on Left Ventricular} Reverse Remodeling after Undersized Mitral Annuloplasty

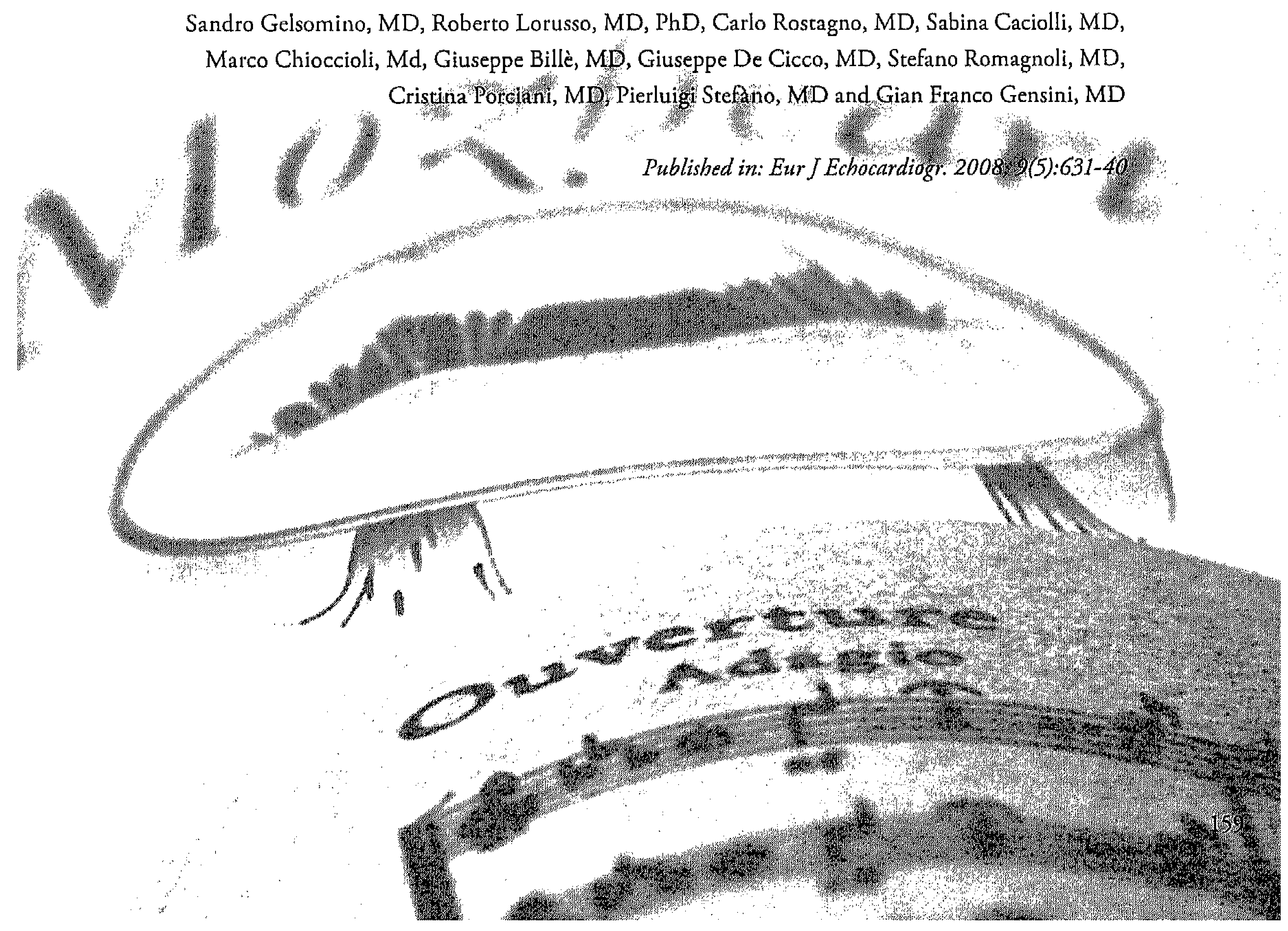


Objective. This study was aimed at exploring the predictive value of Doppler-Derived Mitral Deceleration Time (DT) on left ventricular reverse remodeling (LVRR) in patients with chronic ischemic mitral regurgitation (CIMR) undergoing combined undersized mitral annuloplasty (UMRA) and coronary artery bypass grafting (CABG).

Methods. Two hundred and fifteen patients undergoing combined UMRA and CABG for CIMR berween September 2001 and September 2007 in our Institution were divided into four groups on the basis of baseline DT: Group 1, normal $(n=48)$, Group 2, impaired relaxation $(n=61)$, Group 3, pseudonormal $(n=50)$ and Group 4, restrictive $(n=56)$. Echocardiograms were performed, preoperatively, at discharge and at follow-up appointments (100\% complete, early, median 6 months [interquartile range 4-8 months]) and late, median 38 months (17-61 months).

Results. LVRR, defined as a reduction in ESV $>15 \%$, occurred in $95.7 \%, 96.3 \%, 88.3 \%$ and $0 \%$ in Groups 1, 2, 3 and 4, respectively $(p<0.001)$. Logistic regression analysis showed that DT $\leq 125$ $(p<0.001)$ was a strong predictor of LVRR after annuloplasty. Conclusions. Preoperative assessment of DT adds significant information to commonly used indexes of global and regional function, and represent a very easy and cost effective tool to accurately identify CIMR patients who can really benefit from annuloplasty. 


\section{INTRODUCTION}

The usefulness of Doppler examination of left ventricular (LV) filling based on transmitral flow patterns has been demonstrated in various pathologic conditions ${ }^{1-3}$. Indeed LV filling patterns were studied in patients with dilated cardiomyopathy, acute myocardial infarction and congestive heart failure ${ }^{4-8}$. In addition, its prognostic value has recently been demonstrated in patients undergoing coronary artery bypass (CABG)?.

Combined undersized mitral ring annuloplasty (UMRA) and CABG is a standard surgical approach to relieve chronic ischemic mitral regurgitation $(\mathrm{CIMR})^{10,11}$, although the efficacy is still controversial with many studies revealing a significant recurrence of mitral regurgitation $(M R)^{12-15}$ mainly related to continued LV remodeling and persistent leaflet tethering. Therefore, left ventricular reverse remodeling (LVRR) is considered a significant postoperative target ${ }^{15}$. DopplerDerived Mitral Deceleration Time (DT) has been demonstrated to be a strong predictor of LVRR and survival after myocardial infarction ${ }^{16}$. Nevertheless, the relationship between LV diastolic filling and its change over time and remodeling after reductive annuloplasty, as well as the prognostic impact of serial assessment of LV filling on postoperative LVRR, have not systematically addressed in a large population.

Therefore, the objective of this study was to evaluate the impact of LV filling on reverse remodeling after combined UMRA and CABG for CIMR.

\section{METHODS}

\section{Definitions}

CIMR was defined as the combination of mild-to-severe MR with 1) Prior myocardial infarction $(\mathrm{MI})>16$ days $^{17} ; 2$ ) seventy-five percent or greater stenosis of at least one coronary vessel; 3) a corresponding regional wall motion abnormality; 4) type IIIb leaflet dysfunction following Carpentier's classification ${ }^{3}$ with or without annular dilatation.

Exclusion criteria were: 1) degenerative or other non-ischemic etiology; 2) ischemic isolated type I or type II dysfunction ${ }^{18}$; 3 ) additional mitral valve repair procedures; 4) other valvular or congenital heart diseases.; 5) previous cardiac surgery/percutaneous transluminal coronary angioplasty; 6) atrial fibrillation or sinus rhythm with heart rate at rest $>100 \mathrm{bts} / \mathrm{min}$. 
Among 270 Subjects with CIMR undergoing combined CABG and UMRA in our Institution (Cardiac Surgery, Careggi Hospital, Florence, Italy) between September 2001 and September 2007 and prospectively enrolled in the study, 55 were excluded: 4 had intraoperative annuloplasty failure, 12 showed residual MR (insufficiency $\geq 2+$ following valve repair documented at discharge), 19 died (these were excluded because of unavailable complete data [ 4 early, 15 late]), and 20 had incomplete data. The study population consisted of 215 patients.

\section{FOLLOW UP}

Clinical follow up information was obtained from all survivors through outpatient visits and was $100 \%$ complete. Clinical information regarding New York Heart Association class, recurrence of angina, myocardial infarction, heart failure, reoperation for failed repair was recorded. Median early follow up was 6 months (interquartile range [IQR] 3-8), median late follow up was 38 months (IQR 17-53).

\section{Ethical Issue}

Following the World Medical Association guidelines concerning ethical principles for medical research involving human subjects, the study was approved by Institutional Ethics Board. Furthermore and all patients gave their informed consent. The authors had full access to the data and take responsibility for its integrity. All authors have read and agreed to the manuscript as written.

\section{Echocardiographic Study}

The echocardiographic evaluation was as follows. A transthoracic (TTE) and a transesophageal (TEE) echocardiograms were performed within 5 days before surgery and serial TTE were performed at discharge and at follow-up appointments. Echocardiographic studies were performed on Acuson Sequoia imaging device equipped with a $3.5-\mathrm{MHz}$ ultrasound transducer (Acuson Corporation, Mountain View, CA). Echocardiographic measurements were averaged over three cardiac cycles. Inter-observer (I.C e C.R) and intra-observer variability for measurement of DT, end systolic volume (ESV) and grade of MR were $<5 \%$ (DT, intraobserver $0.7-3.1 \%$, interobserver 0.6 to $4.6 \%$; ESV, intraobserver $0.3-2.9 \%$, interobserver $0.4-4.0 ; \mathrm{MR}$, intraobserver $0.4-2.6 \%$, interobserver 0.3 to $3.4 \%$ ). 
LV volume and left ventricular ejection fraction (LVEF) were assessed by the bi-apical Simpson disk method ${ }^{19}$. The sphericity index (SI) was obtained at end diastole and end systole ( $\mathrm{SI}_{\mathrm{D}}$ and $\mathrm{SI}_{S}$, respectively) as the volume of the left ventricle divided by the volume of a sphere with a diameter equal to the longest axis of the left ventricle measured in the apical view ${ }^{20}$. The wall motion score index (WMSI) was calculated according to a 17-segment model ${ }^{21}$.

\section{Quantitation of MR}

The following quantitative measurements were simultaneously employed to grade the severity of MR and final results were averages of measured values ${ }^{22-23}: 1$ ) Quantitative Doppler. (2) Proximal Isovelocity Surface Area (PISA).

For each measurement, minimum of three cardiac cycles were averaged. The respective thresholds for mild, moderate, and severe MR were $<30,30-59$ and $\geq 60$ $\mathrm{mL} /$ beat for regurgitant volume (RV), $<30,30-49$, and $\geq 50 \%$ for regurgitant fraction $(R F),<20,20-39$, and $\geq 40 \mathrm{~mm}^{2}$ for effective regurgitant orifice (ERO), respectively. In patients with no or trivial MR by color Doppler, $R V$, and $R F$ were used as calculated and ERO was assumed as null. The tenting area (TA) was measured by the area enclosed between the annular plane and mitral leaflets from the parasternal long-axis view at mid-systole. The distance between leaflet coaptation and the mitral annulus plane at early and end systole measured displacement of mitral coaptation toward the LV apex Recurrent MR was the insufficiency $\geq 2+$ at follow up appointments in patients with no/trivial MR at discharge.

\section{Diastolic Filling Patterns}

Diastole was evaluated by measuring transmitral flow. Peak early transmitral flow velocity (E), peak late transmitral flow velocity (A), E/A ratio and deceleration time of $\mathrm{E}$ velocity (DT) were obtained by pulsed Doppler ${ }^{24}$. The isovolumic relaxation time (IVRT) was obtained by continuous wave Doppler with the wave beam directed from the apical five-chamber view across the region between the aortic outflow tract and the inflow tract.

Finally, systolic and diastolic pulmonary venous flow and their ratio $(S / D)$ were routinely assessed ${ }^{24}$.

\section{SURGERY}

Indication for CABG surgery was given following AHA/ACC guidelines ${ }^{25}$. Patients with CIMR showing an ERO $>20 \mathrm{~mm}^{2}$ and $\mathrm{RV}>30 \mathrm{ml}$ were scheduled 
for operation ${ }^{26}$. When MR was $2 / 4$, surgery was indicated: 1 ) in the presence of a dilated LV (end-diastolic volume $\left.>110 \mathrm{~mL} / \mathrm{m}^{2}\right)$ or low $\mathrm{EF}(<0.35 \%)$, as in the case of dilated cardiomyopathy $\left.{ }^{27} ; 2\right)$ in patients with an increase of ERO $>13 \mathrm{~mm}^{2}$ at TTE echocardiographic exercise test $\left.{ }^{28} ; 3\right)$ in ischemic patients with fluctuating MR showing a $M R \geq 3$ after intraoperative loading test ${ }^{29}$.

All patients underwent associated coronary artery bypass grafting. For the purpose of this study, complete revascularization was accomplished when, at least one graft was placed distal to an approximately $50 \%$ diameter narrowing in each of the three major vascular system in which arterial narrowing of this severity was noted in a vessel $\geq 1.5 \mathrm{~mm}$ of diameter. It was not considered necessary to bypass all obstructed diagonal branches of the anterior descending or marginal branches of the circumflex coronary arteries for a classification of complete revascularization. Following this definition $100 \%$ patients underwent complete revascularization.

The ring size was determined by standard measurements of the inter-trigonal distance and anterior leaflet height. A downsizing by two ring sizes was performed in all patients. After cardiopulmonary bypass (CPB), a transesophageal echocardiography (TEE) was performed to assess residual MR: leaflet coaptation $\geq 0.5 \mathrm{~cm}, M R$ $\leq 1$ and systolic MV area $>2 \mathrm{~cm}^{2}$ was assessed as successful repair.

\section{Patient Classification}

According to baseline S/D and DT, irrespective of filling profile, patients were assigned to one of four Group ${ }^{24}$ : Group 1 ( $\mathrm{n}=48$ ), Normal pattern ( $/ \mathrm{D} \geq 1$; DT $<220 \mathrm{msec}$ ); Group 2 ( $\mathrm{n}=61)$, impaired relaxation LV filling pattern $(\mathrm{S} / \mathrm{D} \geq 1 ; \mathrm{DT}>$ $220 \mathrm{msec}$ ); Group 3 ( $\mathrm{n}=50)$, pseudonormal LV filling pattern ( $/ \mathrm{D}<1$; DT 140-200 msec); Group $4(\mathrm{n}=56)$, restrictive LV filling pattern $(S / D<1 ; D T<140 \mathrm{msec})$. The 140 value was chosen because it unequivocally identifies patients with restrictive filling and worse prognosis after myocardial infarction ${ }^{30}$. In no-restrictive patients presenting $\mathrm{E} / \mathrm{A}$ between 1 and 2 , the Valsalva maneuver was performed to unmask the presence of impaired relaxation: the diagnosis of normal diastolic function was confirmed in the absence of reversal of the E/A below 1, during the strain phase of the maneuver ${ }^{31}$.

\section{Primary End-Point}

Based on the same criterion used by Stellbrink et al. ${ }^{32}$, patients were classified as responders of LVRR if the ESV was reduced $>15 \%$ compared with baseline volumes, and as non-responders if ESV was reduced $\leq 15 \%$ at late control following UMRA. 
The sample size was determined by GraphPad StatMate release 2.00 (GraphPad Prism Software, Inc, San Diego, CA) on the basis of preliminary data obtained by echocardiography and was determined on the basis of the following assumptions: Type I error of 0.05 (two-sided), power of $80 \%$, difference in end systolic volume between patients with or without recurrent mitral regurgitation of 0.78 , standard deviation of 2.4. The calculated study population was 200 . However 270 patients were recruited to allow for possible analytic problems while processing the eventualities potentially leading to patient attrition. All data were analyzed with the SPSS for Windows, release 15.0 (SPSS, Inc, Chicago, IL) statistical package and with GraphPad Prism for Windows, release 4.0 (Graph-Pad Prism Software, Inc, San Diego, CA). Variables were tested for normal distribution by the Kolmogorov-Smirnov test. Continuous data were expressed as mean $\pm \mathrm{SD}$ and compared using repeated ANOVA with the Tukey post-hoc test for multiple groups comparison. Comparison of proportions was performed using contingency $\chi^{2}$ analyses or Fisher's test, where appropriate. Non-normally distributed variables were presented as median and interquartile range. The Mann-Whitney and Kruskall-Wallis tests were used to compare non-parametric variables with Dunn's test for multiple comparisons.

Multivariable logistic regression analysis, by means of a backward stepwise algorithm (cut-off for entry 0.05 , for removal 0.10 ) was performed to identify predictors of LVRR. Forty-nine demographic, clinical and Doppler echocardiographic variables were investigated for their predictive value of LVRR. To enhance the accuracy of the model, the number of variables was reduced using variable clustering ${ }^{33}$ until the number of variables to use as candidate in the regression analysis was $\leq \mathrm{m} / 10$, where for binary outcomes, $\mathrm{m}$ is the number of patients in the less frequent outcome category ${ }^{34}$.

Model assumptions (linearity and additivity assumptions) were checked by piecewise cubic polynomials (spiline functions) and pooled interaction test ${ }^{35}$, respectively, and found to be satisfied. Goodness of fit of the final logistic regression models was assessed with the Hosmer-Lemeshow statistic ${ }^{36}$ and predictive accuracy was assessed by the concordance $(c)$ index ${ }^{34,35}$. Internal validation of predictors generated by multivariable logistic regression was performed by means of bootstrapping techniques, with 1000 cycles and generation of $\mathrm{OR}$ and bias corrected $95 \% \mathrm{Cl}^{35}$

Optimal cutoff values were determined as the rounding cut-off that gives the maximum sum of sensitivity and specificity. This value should be the shoulder at the top left of the ROC (receiver operating characteristic curve). Bootstrapping techniques were employed to validate the results.

Cumulative probability for death and re-operation were estimated by use of the Kaplan-Meier method. 
SPSS 12.0 (SPSS, Chicago, IL, USA) and Stats Direct 2.5.7 (Stats Direct, Sale, UK) were used for these calculations. Significance for hypothesis testing was set at the 0.05 two-tailed level.

\section{RESULTS}

\section{Patient Baseline profiles}

As reported in Table 1, preoperative demographic, clinical and intraoperative data were comparable in the four groups. Baseline echocardiographic characteristics of the study population according to preoperative LV diastolic pattern are reported in Table 2. There was no significant difference between the four Groups with respect to quantitative and qualitative MR data. In contrast, patients with restrictive pattern, as compared with those in the other Groups, had larger coaptation height $(p=0.001), L V$ diameters $(p=0.03)$ and volumes (ESV, $p<0.001 ; E D V, p=0.04), a$ more spherical $(\mathrm{p}<0.001)$ and dysfunctioning LV (WMSI, $\mathrm{p}<0.001$; LVEF, $\mathrm{p}=0.04$ ). Furthermore, they had lower DT and $S / D$, by definition $(p<0.001)$ with higher $E / A$ ratio $(\mathrm{p}=0.04)$ and shorter IVRT $(\mathrm{p}=0.02)$.

\section{CHANGES IN ECHOCARDIOGRAPHIC VARIABLES}

Echocardiographic results are shown in Table 3. Mitral regurgitation was significantly reduced at discharge in all groups $(\mathrm{p}<0.001)$ and it did not change at following controls in Groups 1, 2 and 3. In contrast, in patients with restrictive filling pattern, it remained stable at early control, whereas increased significantly at late control $(p<0.001)$. Indeed, at late follow-up, among patients in group $4,59.1 \%$ $(n=26)$ had an MR grade $\geq 2$ vs. $8.3 \%(n=4), 9.8 \%(n=6)$ and $10 \%(n=5)$ in Groups 1,2 , and 3 , respectively $(\mathrm{p}<0.001)$.

In normal, impaired relaxation and pseudonormal groups, ESV decreased significantly at discharge $(p<0.001)$, early $(p<0.001)$ and late controls $(p<0.001)$. In contrast, in the restrictive group, ESV was significantly reduced at discharge ( $p$ $=0.01)$ and early follow-up $(p=0.02)$ but increased again $(p<0.001)$ at late control. A $15 \%$ reduction in ESV (considered indicative of LVRR) occurred in $95.7 \%, 96.3 \%$, $88.3 \%$ and $0 \%$ in Groups $1,2,3$, and 4 , respectively $(p<0.001)$. A further comparison of the degree of LV reverse remodeling in the three groups showed that ESV was reduced $20 \%$ or more in 18 patients $(40.0 \%)$ and $<20 \%$ in 27 patients $(60.0 \%)$ in Group 1. In Group 2, 33 patients (62.3\%) had a reduction in ESV $<20 \%$ and 20 patients $(37.3 \%)$ had a reduction $\geq 20 \%$. In addition, ESV was reduced $20 \%$ or more in 13 patients (34.2\%) and $<20 \%$ or less in 25 patients $(65.8 \%)$ in Group 3 (between groups $p=0.71$ ). Finally, among patients with a restrictive pattern ESV was reduced 
Table 1. Patients profile $(n=215)$

\begin{tabular}{|c|c|c|c|c|c|}
\hline & $\begin{array}{l}\text { Group 1 } \\
\mathrm{n}=48\end{array}$ & $\begin{array}{l}\text { Group } 2 \\
\mathrm{n}=61\end{array}$ & $\begin{array}{l}\text { Group } 3 \\
n=50\end{array}$ & $\begin{array}{l}\text { Group } 4 \\
n=56\end{array}$ & $\mathbf{p}$ \\
\hline Age (years) & $69 \pm 7$ & $68.5 \pm 8$ & $68 \pm 5$ & $69 \pm 8$ & 0.8 \\
\hline Gender $\mathrm{M} / \mathrm{F}$ & $\begin{array}{l}31 / 17 \\
(64.6 / 35.4)\end{array}$ & $\begin{array}{l}38 / 23 \\
(62.2 / 37.8)\end{array}$ & $\begin{array}{l}32 / 18 \\
(64.0 / 36.0)\end{array}$ & $\begin{array}{l}35 / 21 \\
(62.5 / 37.5)\end{array}$ & 0.53 \\
\hline Family history & $22(45.8)$ & $28(45.9)$ & $23(46.0)$ & $27(48.2)$ & 0.82 \\
\hline \multicolumn{6}{|l|}{ Euroscore } \\
\hline Additive & $8.0[5-9]$ & $8.3[6-11]$ & $8.0[5-9]$ & $8.7[5-8]$ & 0.4 \\
\hline Logistic & $14.8[11-18]$ & $14.6[10-17]$ & $15.0[13-18]$ & $15.1[10-19]$ & 0.8 \\
\hline COPD & $10(20.8)$ & $8(13.1)$ & $9(18.0)$ & $9(16.1)$ & 0.07 \\
\hline Chronic renal disease & $9(18.7)$ & $13(21.3)$ & $11(22.0)$ & $10(17.8)$ & 0.16 \\
\hline Cerebral vascular disease & $6(12.5)$ & $9(14.0)$ & $8(16.0)$ & $6(10.7)$ & 0.43 \\
\hline Peripheral vascular disease & $4(8.3)$ & $6(9.8)$ & $3(6.0)$ & $4(7.1)$ & 0.64 \\
\hline Familiar history & $40(47.6)$ & & & & \\
\hline Systemic hypertension & $20(41.6)$ & $24(39.3)$ & $19(38.0)$ & $23(41.0)$ & 0.79 \\
\hline Diabetes mellitus & $14(29.1)$ & $17(27.8)$ & $13(26.0)$ & $15(26.7)$ & 0.88 \\
\hline Hyperlipidemia & $20(41.6)$ & $25(40.9)$ & $20(40.0)$ & $22(39.2)$ & 0.9 \\
\hline \multicolumn{6}{|l|}{ Myocardial infarction } \\
\hline Inferior/Posterior & $25(52.1)$ & $29(49.2)$ & $25(50.0)$ & $29(51.7)$ & \\
\hline Anterior/Septal & $4(8.3)$ & $5(8.5)$ & $4(8.0)$ & $4(7.1)$ & \\
\hline Lateral & $9(18.8)$ & $11(18.6)$ & $9(18.0)$ & $10(17.9)$ & 0.6 \\
\hline Combined & $10(20.8)$ & $14(23.7)$ & $12(24.0)$ & $13(23.3)$ & \\
\hline Angina & $25(52.0)$ & $49(47.5)$ & $23(46.0)$ & $28(50.0)$ & 0.46 \\
\hline CCS Angina class & $2[1-3]$ & $2[1-3]$ & $2[1-3]$ & $2[1-3]$ & $>0.9$ \\
\hline \multicolumn{6}{|l|}{ NYHA } \\
\hline$\leq \mathrm{II}$ & $11(22.9)$ & $13(21.3)$ & $10(20.0)$ & $11(19.6)$ & \\
\hline III & $30(62.5)$ & $38(62.3)$ & $32(64.0)$ & $36(64.3)$ & 0.4 \\
\hline IV & $7(14.6)$ & $10(16.4)$ & $8(16.0)$ & $9(16.1)$ & \\
\hline Coronary vessel disease & $3[1-3]$ & $3[1-3]$ & $3[1-3]$ & $3[1-3]$ & $>0.9$ \\
\hline 1 & $3(6.3)$ & $4(6.6)$ & $4(8.0)$ & $3(5.4)$ & \\
\hline 2 & $19(39.6)$ & $23(37.7)$ & $21(42.0)$ & $25(44.6)$ & \\
\hline$\geq 3$ & $26(54.1)$ & $34(55.7)$ & $25(50.0)$ & $28(50.0)$ & \\
\hline Left Main & $8(16.6)$ & $10(16.3)$ & $7(14.0)$ & $9(16.0)$ & \\
\hline Left anterior descending & $44(91.7)$ & $56(91.8)$ & $44(88.0)$ & $52(92.8)$ & \\
\hline Circumflex system & $30(62.5)$ & $36(59.0)$ & $27(54.0)$ & $33(58.9)$ & \\
\hline Right coronary system & $38(79.1)$ & $47(77.0)$ & $39(78.0)$ & $43(76.7)$ & \\
\hline
\end{tabular}




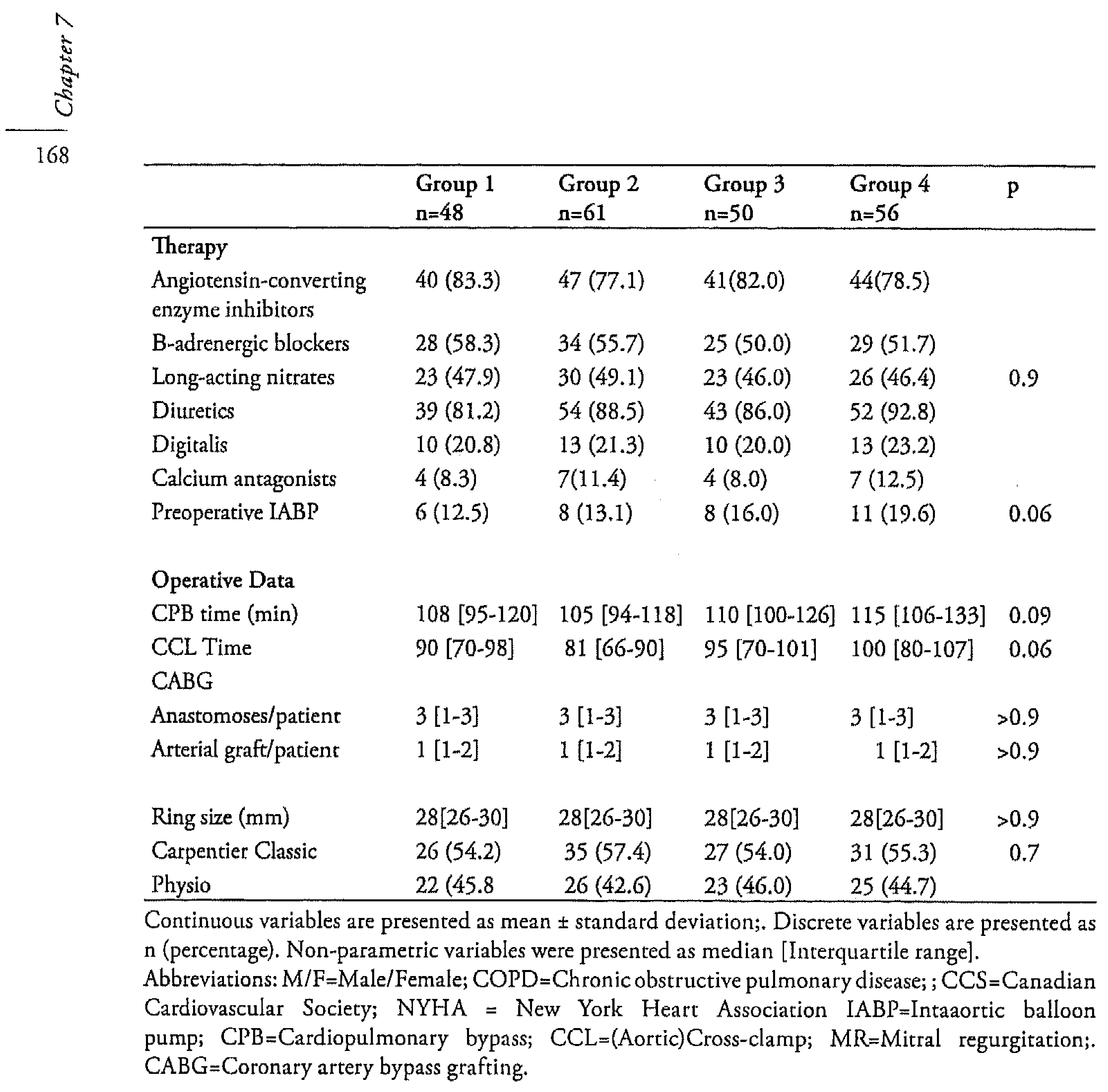


Prognostic Value of Doppler-Derived Mitral Deceleration Time

Table 2. Baseline echocardiographic findings

\begin{tabular}{|c|c|c|c|c|c|}
\hline & $\begin{array}{l}\text { Group } 1 \\
n=48\end{array}$ & $\begin{array}{l}\text { Group } 2 \\
\mathrm{n}=61\end{array}$ & $\begin{array}{l}\text { Group } 3 \\
\mathrm{n}=50\end{array}$ & $\begin{array}{l}\text { Group } 4 \\
\mathrm{n}=56\end{array}$ & $\mathrm{p}$ \\
\hline MR Grade & $3[3-4]$ & $3[3-4]$ & $3[3-4]$ & $3[3-4]$ & $>0.9$ \\
\hline $2+$ & $8(16.6)$ & $10(16.4)$ & $8(16 . .0)$ & $10(17.8)$ & \\
\hline $3+$ & $27(56.3)$ & $34(55.8)$ & $28(56.0)$ & $31(55.4)$ & \\
\hline $4+$ & $13(27.1)$ & $17(27.8)$ & $14(28.0)$ & $15(26.8)$ & \\
\hline \multicolumn{6}{|l|}{ MR Quantitative data } \\
\hline TA & $3.5 \pm 1.1$ & $3.7 \pm 1.0$ & $3.2 \pm 1.1$ & $3.5 \pm 1.1$ & 0.6 \\
\hline ERO & $37 \pm 12$ & $36 \pm 11$ & $38 \pm 14$ & $40 \pm 16$ & 0.42 \\
\hline RF & $46 \pm 13$ & $44 \pm 12$ & $47 \pm 13$ & $50 \pm 18$ & 0.55 \\
\hline RV & $57 \pm 17$ & $56 \pm 15$ & $58 \pm 18$ & $60 \pm 16$ & 0.72 \\
\hline $\mathrm{CH}$ & $1.0 \pm 0.2$ & $1.2 \pm 0.2$ & $1.2 \pm 0.2$ & $1.4 \pm 0.2$ & 0.001 \\
\hline \multicolumn{6}{|c|}{ Direction of regurgitant jet } \\
\hline Central & $15(31.2)$ & $19(31.1)$ & $16(32.0)$ & $19(33.9)$ & \\
\hline Anterior & $6(12.5)$ & $8(13.1)$ & $6(12.0)$ & $6(10.7)$ & 0.7 \\
\hline Posterior & $11(22.9)$ & $14(23.0)$ & $11(22.0)$ & $14(25.1)$ & \\
\hline Complex & $16(33.4)$ & $68(32.8)$ & $17(34.0)$ & $17(30.3)$ & \\
\hline \multicolumn{6}{|c|}{ Carpentier's classification } \\
\hline Type IIIb & $30(62.5)$ & $39(63.9)$ & $31(62.0)$ & $37(66.1)$ & 0.9 \\
\hline $\begin{array}{l}\text { Type IIIb + annular } \\
\text { dilatation }\end{array}$ & $18(37.5)$ & $22(36.1)$ & $19(38.0)$ & $19(33.9)$ & $>0.9$ \\
\hline \multicolumn{6}{|c|}{ Systolic function and Geometry } \\
\hline EDD & $59 \pm 6$ & $60 \pm 7$ & $56 \pm 7$ & $65 \pm 8$ & 0.03 \\
\hline ESD & $49 \pm 7$ & $51 \pm 8$ & $47 \pm 7$ & $56 \pm 9$ & 0.03 \\
\hline LA & $49 \pm 6$ & $50 \pm 7$ & $48 \pm 3$ & $47 \pm 7$ & 0.7 \\
\hline ESV & $109 \pm 22$ & $110 \pm 24$ & $112 \pm 31$ & $140 \pm 26$ & $<0.001$ \\
\hline EDV & $176 \pm 37$ & $165 \pm 35$ & $176 \pm 28$ & $194 \pm 30$ & 0.04 \\
\hline LVEF & $38 \pm 12$ & $33 \pm 12$ & $36 \pm 10$ & $27 \pm 11$ & 0.007 \\
\hline $\mathrm{SI}_{\mathrm{S}}$ & $0.56 \pm 0.1$ & $0.55 \pm 0.2$ & $0.52 \pm 0.1$ & $0.75 \pm 0.1$ & $<0.001$ \\
\hline$S I_{D}$ & $0.64 \pm 0.1$ & $0.62 \pm 0.1$ & $0.62 \pm 0.1$ & $0.80 \pm 0.1$ & $<0.001$ \\
\hline WMSI & $1.10 \pm 0.5$ & $1.14 \pm 0.7$ & $1.15 \pm 0.6$ & $1.71 \pm 0.7$ & $<0.001$ \\
\hline \multicolumn{6}{|l|}{ Diastolic function } \\
\hline E/A ratio & $1.1 \pm 0.3$ & $0.6 \pm 0.08$ & $1.5 \pm 0.3$ & $1.9 \pm 0.6$ & 0.04 \\
\hline DT & $215 \pm 25$ & $235 \pm 28$ & $200 \pm 21$ & $119 \pm 12$ & $<0.001$ \\
\hline IVRT & $95 \pm 7$ & $112 \pm 9$ & $95 \pm 10$ & $54 \pm 10$ & 0.02 \\
\hline$S / D$ & $1.1 \pm 0.3$ & $1.0 \pm 0.2$ & $0.93 \pm 0.1$ & $0.7 \pm 0.1$ & $<0.001$ \\
\hline
\end{tabular}

Continuous variables are presented as mean \pm standard deviation;. Non-parametric variables were presented as median [Interquartile range]. Abbreviations: $M R=$ Mitral Regurgitation; $T A=T e n t i n g$ area $\left(\mathrm{cm}^{2}\right) ; \mathrm{ERO}=$ effective regurgitant orifice $\left(\mathrm{mm}^{2}\right) ; \mathrm{RF}=$ Regurgitant fraction (\%); RV=Regurgitant volume (ml/beat); $\mathrm{CH}=$ Coaptation height $(\mathrm{cm}) ; \mathrm{MA}_{\mathrm{s}}=\left(\right.$ Systolic) Mitral annular area $\left(\mathrm{cm}^{2}\right) ; \mathrm{MA}_{\mathrm{d}}=($ Diastolic $)$ Mitral annular area $\left(\mathrm{cm}^{2}\right) ; E D D=($ Left ventricular) End diastolic diameter $(\mathrm{mm}) ; \mathrm{ESD}=($ Left ventricular) End systolic diameter (mm); LA=Left atrium (mm); ESV=(Left ventricular) End systolic volume $(\mathrm{ml}) ; \mathrm{EDV}=($ Left ventricular) End diastolic volume $(\mathrm{ml}) ; \mathrm{LVEF}=\mathrm{Left}$ ventricular ejection fraction (\%); $\mathrm{SPI}_{\mathrm{S}}=$ Systolic Sphericity Index ; $\mathrm{SPI}_{\mathrm{D}}=$ Diastolic Sphericity Index WMSI=Wall motion Score Index; $\mathrm{E}=$ Peak trans-mitral flow velocity; $\mathrm{A}=$ peak late trans-mitral flow velocity; $\mathrm{DT}=$ =Deceleration time at early diastolic filling (ms); IVRT=Isovolumetric relaxation time (ms); S/D Systolic to diastolic pulmonary venous flow ratio; $n s=$ not significant. 


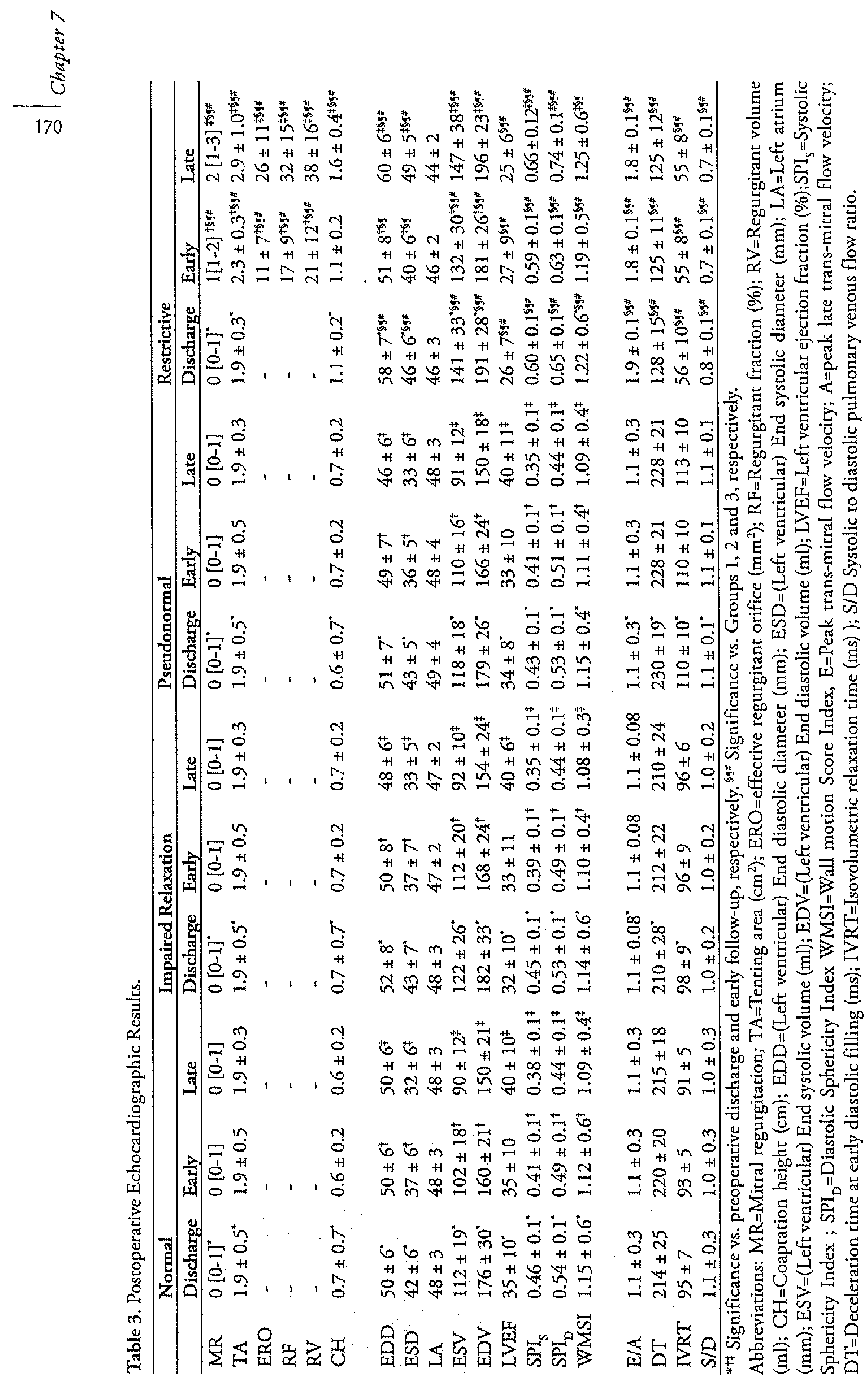


Table 4. Independent predictors of LV reverse remodeling.

\begin{tabular}{lllll}
\hline & & & \multicolumn{2}{c}{$95 \%$ Bias-corrected CI } \\
\cline { 4 - 5 } Variable & Odds Ratio & $\mathrm{P}$ & Lower Limit & Upper Limit \\
\hline $\mathrm{DT}$ & 3.08 & $<0.001$ & 1.54 & 5.90 \\
$\mathrm{SI}_{\mathrm{S}}$ & 2.40 & 0.001 & 1.48 & 3.99 \\
$\mathrm{WMSI}$ & 1.02 & 0.008 & 1.00 & 2.02 \\
$\mathrm{~S} / \mathrm{D}$ & 1.22 & 0.06 & 0.87 & 2.13 \\
$\mathrm{SI}$ & 0.98 & 0.2 & 0.09 & 1.93 \\
$\mathrm{LVEF}$ & 0.93 & 0.24 & 0.08 & 1.78 \\
$\mathrm{ESD}$ & 0.81 & 0.33 & 0.09 & 1.01 \\
$\mathrm{EDD}$ & 0.87 & 0.11 & 0.09 & 1.23 \\
$\mathrm{E} / \mathrm{A}$ & 0.67 & 0.62 & 0.07 & 1.0 \\
IVRT & 0.51 & 0.73 & 0.09 & 1.2 \\
\hline
\end{tabular}

Abbreviations: LV=Left Ventricle; DT=Deceleration time SIS=Systolic Sphericity Index ; S/D Systolic to diastolic pulmonary venous flow ratio.;WMSI=Wall Motion Score Index; $\mathrm{SI}_{\mathrm{D}}=$ Diastolic Sphericity Index; $L V E F=L e f t$ ventricular ejection fraction; $E S D=$ (Left ventricular) End systolic diameter $(\mathrm{mm})$; $\mathrm{EDD}=($ Left ventricular) End diastolic diameter; $\mathrm{E}=$ Peak trans-mitral flow velocity; $\mathrm{A}=$ peak late transmitral flow velocity; at early diastolic filling; IVRT=Isovolumetric relaxation time.

$10 \%$ or more in one patient $(2.2 \%),<10 \%$ in 27 patients $(61.4 \%)$, relatively stable $( \pm$ $1 \%$ ) in four patients (9.1\%), and enlarged in 12 patients (27.3\%; mean increment 3.9 $\pm 1.7 \%)$. Changes in EDV, ESD, EDD and WMSI mirrored those of ESV.

Systolic and diastolic sphericity indexes improved in Groups 1, 2, and 3 at discharge $\left(p<0.001\right.$ ), early (Group 1, $p=0.01$ for both $\mathrm{SI}_{S}$ and $\mathrm{SI}_{\mathrm{D}}$; Group $2, p<0.001$ for $\mathrm{SI}_{S}$ and $p=0.02$ for $\mathrm{SI}_{\mathrm{D}}$; Group 3, $p=0.04$ for both $\mathrm{SI}_{\mathrm{S}}$ and $\left.\mathrm{SI}_{\mathrm{D}}\right)$ as well as at late control (Group 1, $p=0.03$ for $\mathrm{SI}_{\mathrm{S}}$ and $p=0.01$ for $\mathrm{SI}_{\mathrm{D}}$; Group 2, $p=0.02$ for $\mathrm{SI}_{\mathrm{S}}$ and $p=0.01$ for $\mathrm{SI}_{\mathrm{D}}$; Group 3, $p<0.001$ for both $\mathrm{SI}_{\mathrm{S}}$ and $\mathrm{SI}_{\mathrm{D}}$ ). In Group 4 there was an initial, but not significant, reduction in both the systolic and diastolic sphericity indexes, which remained stable at early follow-up with a late increase exceeding the preoperative value $(p<0.001)$. In normal, impaired relaxation, and pseudonormal groups, LVEF significantly improved at discharge and at late follow-up, remaining unchanged at early control. In contrast, in the restrictive group it did not significantly change at any study point.

Indices of diastolic function remained stable over time in patients with baseline restrictive filling. In patients with pseudormal filling $E / A$ increased $(p=0.03)$ whereas DT $(p<0.001)$, IVRT $(p=0.006)$ and S/D $(p=0.04)$ reduced ad discharge. All these values remained constant afterwards. In patients with impaired relaxation, E/A reduced $(\mathrm{p}=0.02)$, whereas DT $(\mathrm{p}=0.001)$ and IVRT $(\mathrm{p}=0.03)$ raised at discharge without further changes at subsequent controls. $S / D$ did not show significant changes in this Group. Index of diastolic function did not modify over time in patients with normal LV filling pattern. 


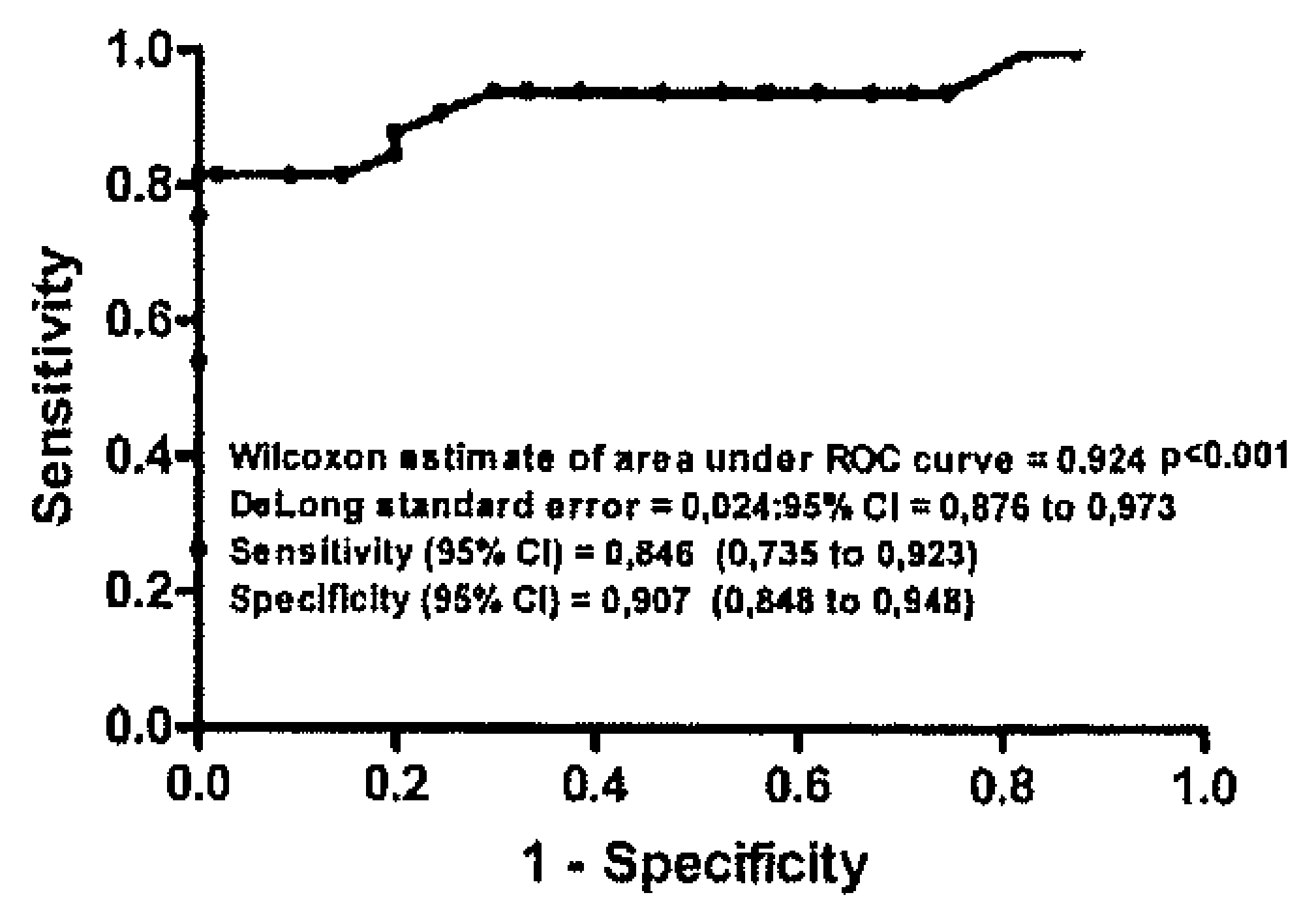

Figure 1. Receiver operating characteristic (ROC) curve for Doppler-Derived Mirral Deceleration Time (DT) to predict LV remodeling.

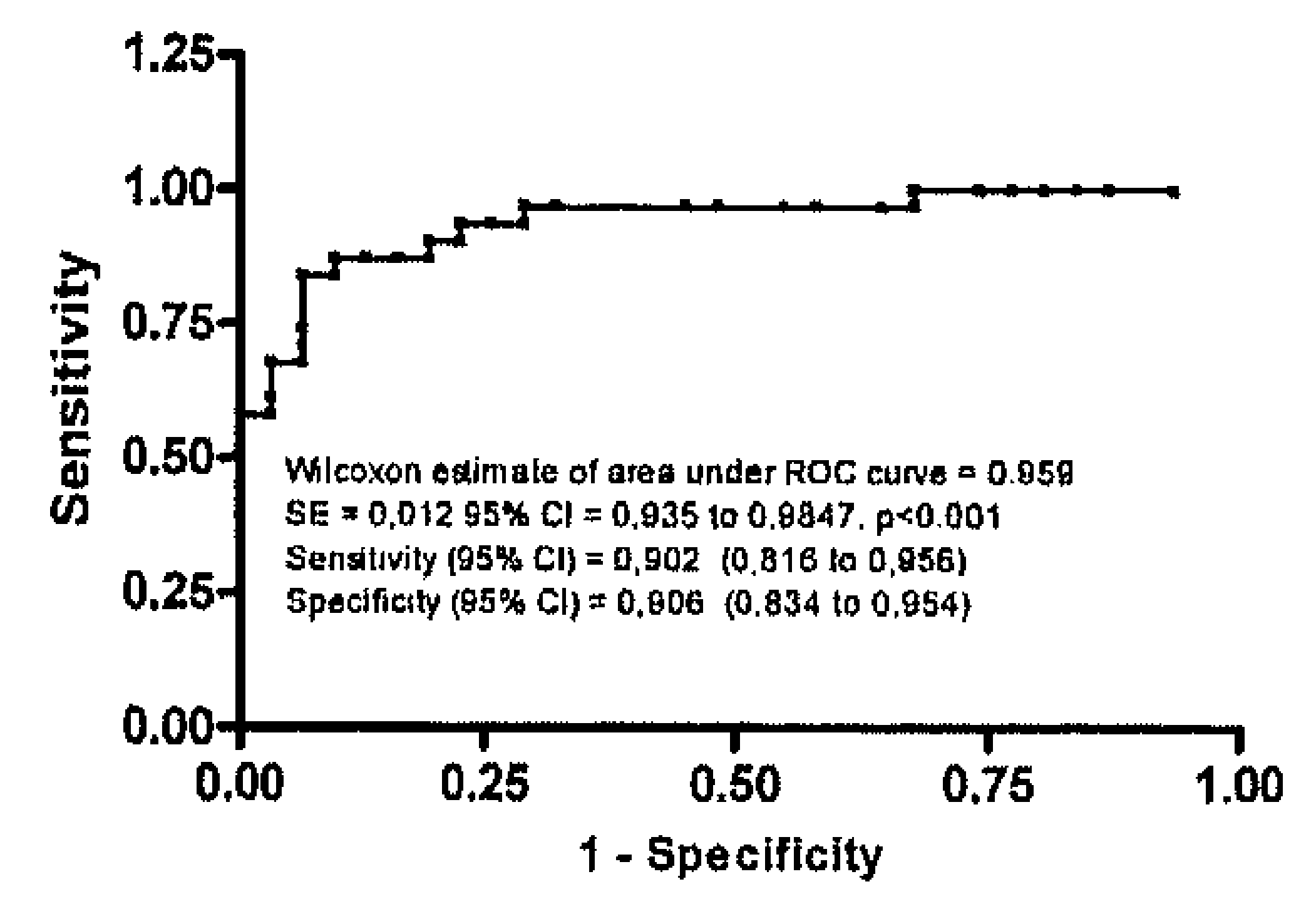

Figure 2. Receiver operating characteristic (ROC) for systolic sphericity index $\left(\mathrm{SI}_{\mathrm{s}}\right)$ to predict LV remodeling.

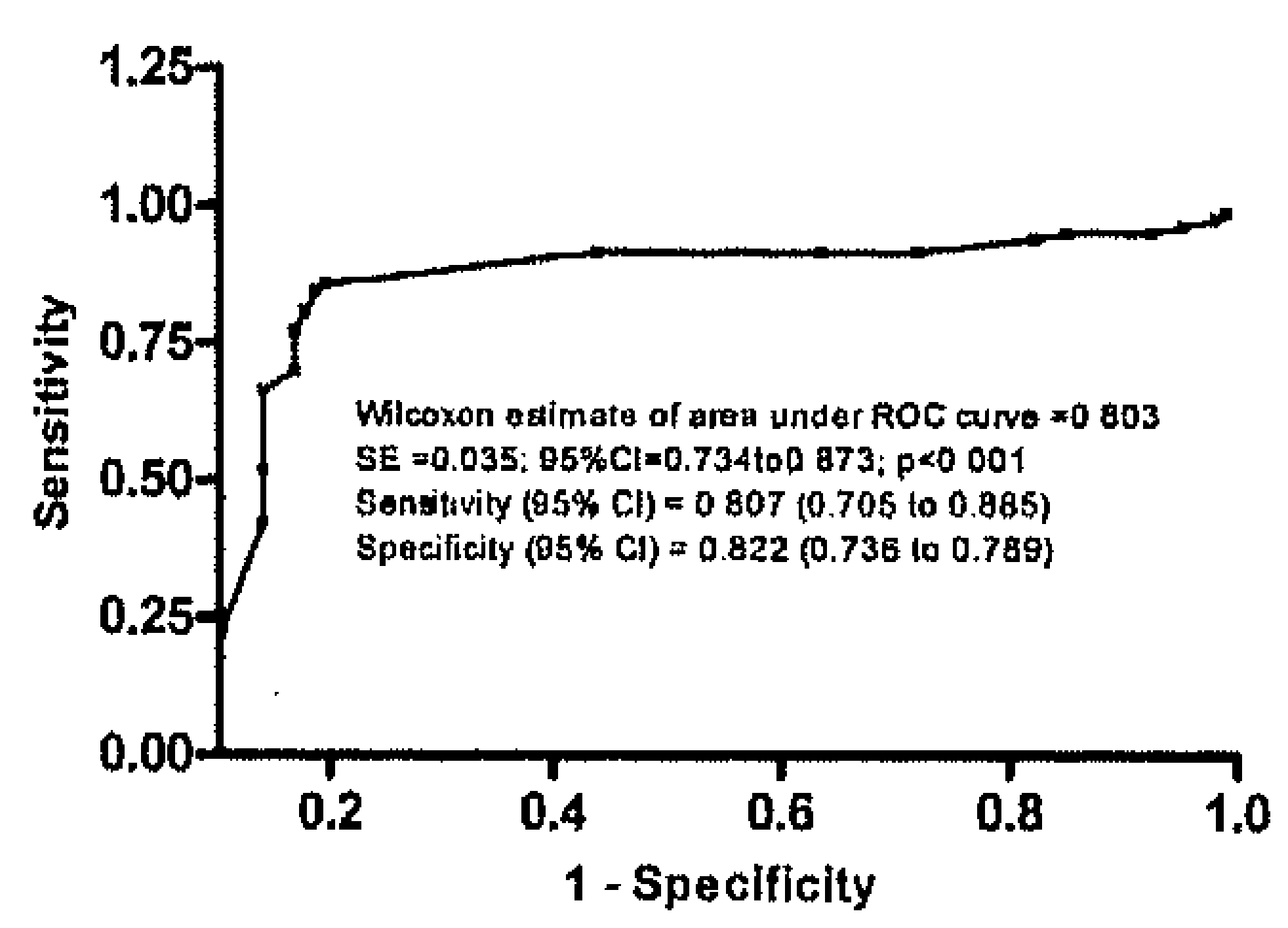

Figure 3. Receiver operating characteristic (ROC) for Wall Motion Score Index (WMSI) to predict LV remodeling. 
By multiple regression analysis baseline DT, $S I_{s}$ and WMSI resulted as independent predictors of LVRR. Internal validation of such multivariable analysis by means of bootstrapping, including the same variables of standard logistic regression model, further confirmed the significant predictive of LVRR (bootstrap $O R=2.2$, 95\% bias-corrected $95 \% \mathrm{CI}=1.0-4.9$ ). Finally, logistic regression model was reliable (Hosmer-Lemeshow test, $\mathrm{p}=0.58$ ) and accurate ( $\mathrm{c}$ index $=0.77$ ).

ROC curve analysis to determine optimal cutoff values (Figure 1-3) to predict reverse remodeling showed the following values: DT $\leq 125 \mathrm{~ms}$ ( $95 \%$ CI by bootstrapping $108-171 \mathrm{~ms}), \mathrm{SI}_{\mathrm{s}}$ had $90 \%$ of sensitivity and specificity with an optimal cutoff $\leq 0.7$ ( $95 \% \mathrm{CI}$ by bootstrapping $0.58-0.81$ ), WMSI $<1.5$ (95\% CI by bootstrapping $1.35-1.62)$

\section{Clinical Follow up}

At late control, mean NYHA class was $2.1 \pm 0.3,2.3 \pm 0.4,2.2 \pm 0.4$ and $3 \pm$ 0.6 in Groups 1, 2, 3 and 4, respectively $(\mathrm{p}<0.001) .70 .5 \%$ patients in the Group 4 had NYHA $\geq 3 \%$ vs. $33.3 \%$ in the pseudonormal group ( $<<0.001), 18.1 \%$ in the impaired relaxation group $(\mathrm{p}<0.001)$ and $4.6 \%$ in the normal Group $(\mathrm{p}<0.001)$.

Seventeen patients underwent re-operation for failed repair (four had a re-repair and 13 underwent mitral valve replacement): none in Group 1, two in Group 2, six in Group 3 and 9 in Group 4. Five-year freedom from reoperation for failed repair were $100 \%, 94 \pm 5 \%, 67 \pm 9 \%$ and $43 \pm 7 \%$ in Groups 1-4, respectively $(\mathrm{p}<0.001)$. None of the patients developed clinical signs of angina. Furthermore, no myocardial infarction occurred in the postoperative period

\section{DISCUSSION}

The present study demonstrates that a baseline restrictive filling pattern, as expressed by a short DT, identifies patients who are ultimately candidates to continued LV remodeling after UMRA leading to mitral repair failure and recurrence of mitral regurgitation. Ereminiene et $\mathrm{a}^{37}$ demonstrated, in 55 patients, that diastolic restrictive filling pattern and deceleration time were independent predictors of death and residual moderate MR late (12 months) after ischemic mitral valve repair. Nonetheless these authors defined the restrictive filling pattern as an E/A ratio $>2$ or an E/A ratio $1-2$ with DT $<150 \mathrm{msec}$ and IVRT $<60 \mathrm{~ms}$. E and E/A ratio are partially dependent on age, heart rate, loading conditions and degree of MR. In particular, mitral regurgitation may increase the E-wave velocity of transmitral flow, which resembles pseudonormal or restrictive patterns. Several studies have shown that a 
short DT, even not load independent, irrespective of the ratio of early and late filling velocity $(\mathrm{E} / \mathrm{A})$ ratio, is strongly related to pulmonary capillary wedge pressure ${ }^{38,39}$ and end-diastolic pressure ${ }^{40}$ and it is an useful parameter for the serial assessment of LV diastolic function in post infarct patients also in those with $M^{41}$. For this reason, we used DT as a means of assessing LV filling, irrespective of filling pattern. Nonetheless, in an attempt to overcome the limitations of transmitral flow Doppler indices, we used routinely the assessment of pulmonary venous flow. On the basis of these two indices we classified patients in four Groups: Normal, impaired relaxation, pseudonormal and restrictive.

Furthermore, we employed the Valsalva maneuver in no-restrictive patients presenting E/A between 1 and 2, to unmask the presence of impaired relaxation.

Patients belonging to restrictive Group had larger distance between the coaptation point and the mitral annulus (coaptation height, $\mathrm{p}=0.001$ ), which represents a quantitative estimate of incomplete mitral closure. In a recent study Karagiannis et al. ${ }^{42}$ showed a linear correlation between coaptation height and both measurements of left ventricular size and indices of systolic performance of the left ventricle. In our experience, subjects with restrictive diastolic pattern had larger diameters $(p=0.03)$ and volumes (ESV, $\mathrm{p}<0.001 ; \mathrm{EDV}, \mathrm{p}=0.04$ ) and a more dysfunctioning LV (LVEF, $\mathrm{p}$ $=0.04$ ) and this might explain the larger coaptation height found in this Group.

Cardiac remodeling is a well known important aspect of CIMR and myocardial disease progression responsible for recurrence of MR after annuloplast $y^{13,14}$. Thus left ventricular reverse remodeling (LVRR) is considered a primary postoperative target. Following the same criterion used by Stellbrink et a ${ }^{31}$, a reduction $>15 \%$ in ESV compared with baseline volumes was considered LVRR. Other Groups ${ }^{41}$ considered LV end diastolic dimension as significant index of LVRR. Nonetheless, measurements of LV volumes have been demonstrated to be more reliable than LV diameters in assessing LV remodeling, especially in enlarged ventricles ${ }^{43}$. On this basis, LV reverse remodeling occurred in $95.7 \%, 96.3 \%, 88.3 \%$ and $0 \%$ in Groups $1,2,3$, and 4 , respectively $(p<0.001)$. In addition, among restrictive patients, 12 $(27.3 \%)$ showed continuous remodeling with a mean increment in ESV of $3.9 \pm$ $1.7 \%$ at late examination.

Nonetheless, EDV and ESV decreased at early follow-up also in the restrictive group. Thus, increase in EDV and ESV at late follow-up could be related to the increasing or recurrence of a significant $M R$ and all this might be inducing the restrictive pattern and not vice versa, since from the beginning the restrictive group has larger volumes and lower LVEF. A DT value $>125 \mathrm{~ms}$ was the most powerful independent predictor of LVRR whereas, interestingly, LVEF did not provide any prognostic value thus indicating that LV diastolic dysfunction may be more sensitive than LV systolic dysfunction in predicting LVRR after CABG and restrictive annuloplasty. Nonetheless, the lack of improvement in LVEF in the restrictive group could be related to the changes in EDV and ESV in presence of a recurrence of MR. 
In other words, the fact that LVEF did not show any prognostic value might be due to the presence of $M R$ in this population.

Other predictors of LVRR were $\mathrm{SI}_{\mathrm{S}}(\mathrm{p}<0.001)$ and WMSI $(\mathrm{p}<0.008)$. By comparing the four groups in our study, it appears evident that patients with preoperative restrictive filling pattern were much more globally remodeled at every study point; at late control they still showed a more spherical and enlarged LV. Particularly, the late increase in sphericity indexes in this group, which exceeded the preoperative value, was consistent with further remodeling after UMRA and reflects increased tethering on the mitral valve, thus translating into a significantly higher number of patients with late significant MR in this group $(p<.001)$.

\section{Study LIMITATIONS}

Our study findings should be viewed in light of some inherent limitations.

- Similarly to other studies ${ }^{37}$ we employed conventional indices of LV diastolic function.

- Tissue Doppler imaging (TDI) would have been more useful in estimating LV diastolic filling pattern. Nonetheless, DT, even not load independent is strongly related to pulmonary capillary wedge pressure and end-diastolic pressure and it is an useful parameter for the serial assessment of LV diastolic function in post infarct patients also in those with MR. Furthermore, in an attempt to overcome the limitations of transmitral flow Doppler indices, we used routinely the assessment of pulmonary venous flow. An evaluation of diastolic function after UMRA and CABG, employing TDI will be ongoing to confirm these findings.

- Evaluation of LVRR was based on volumes obtained by echocardiography; Volumetry by $2 \mathrm{D}$ echocardiography depends on geometric assumptions and is subject to image-plane positioning errors. Hence it is not accurate in left ventricles that are distorted in shape such as after myocardial infarction. However, this limitation belongs to most of published paper regarding this pathology.

- Viability testing was not performed in these patients. Therefore lack of LVRR in non responders might be also due to irreversible ischemic myocardial damage (not-viable myocardium). This issue deserves further investigation.

- Postoperative evaluation of the coronary status was not assessed. It would have been helpful to differentiate between surgical failure (valve repair and CABG) and the progress of the coronary disease.

- Fifteen patients with late deaths were not included in the study. They could have benefited from serial echocardiograms possibly with different results.

- Estimated cutoffs are known to be very susceptible to changes in the study population. 
We employed bootstrapping techniques to validate the results, nonetheless, it has also been documented that the sensitivity/specificity associated with these cutoffs are overly optimistic.

\section{STRENGTH OF THE STUDY}

This is a large cohort reported with UMRA in CIMR patients with detailed (100\% complete) echocardiographic follow-up. Furthermore our patient cohort was more homogeneous than in other study and does not display common clinical and methodological pitfalls that limit results from many studies of mitral valve repair ${ }^{44}$ : all patients underwent associated $C A B G$, they had no concomitant $M V$ procedures, and the entire cohort was uniform regarding the MV ischemic leaflet dysfunction. Moreover, to assess results for MV repair, we studied true "recurrent" MR excluding those patients with "residual" $M R$ in whom the insufficiency was presumably never eliminated at surgery. Additionally, we undertook valve sizing in a standardized fashion and the degree of undersizing was homogeneous over the 5-year-period of the study. Finally, we used only two rings (Carpentier's rigid or semi-flexible rings).

\section{Clinical Implications.}

In our experience combined CABG and UMRA do not ensure successful and durable elimination of MR and significant LVRR in all patients. Those who can benefit from this approach could be preoperatively identified utilizing echo predictors. However, restrictive annuloplasty resulted to be ineffective in a large percentage of patients and results of this study suggest the need for different approaches directly addressing ventricular tethering in most of CIMR patients ${ }^{45}$. These procedures were introduced in the recent years in clinical practice, thus our experience with these techniques is actually in the preliminary phase. Furthermore, mitral valve replacement, largely employed in the past in ischemic regurgitation, although eliminates the short-term risk of recurrent $M R$, it is associated with poor long-term survival ${ }^{46}$. Thus, in our actual policy, we do not consider MV replacement as a reasonable alternative to repair.

Another interesting finding of our study, apart from the predictive value of DT on LVRR, was that in patients with more advanced diastolic dysfunction, evidenced by a LV restrictive filling, UMRA associated to CABG did not cause improvement of diastolic function. Contrastingly, an improvement of diastolic function occurred in no-restrictive patients. This suggest that an early surgical indication is advisable in patients with CIMR in less severe stages of disease. In the presence of advanced diastolic dysfunction, patients should be considered poor candidates for this procedure and concomitant or alternative procedure should be contemplated. 


\section{CONCLUSIONS}

Preoperative assessment of DT adds significant information to commonly used indexes of global and regional function, and represents a very easy and cost effective tool to accurately identify CIMR patients who can really benefit from annuloplasty. Long term prospective studies on this controversial issue are encouraged.

\section{REFERENCES}

1. De Simone $G$, Greco $R$, Mureddu $G$ et al. Relation of left ventricular diastolic properties to systolic function in arterial hypertension.Circulation. 2000;101(2):152-7.

2. Shingu $Y$, Shiiya N, Mikami T, Matsuzaki K, Kunihara T, Marsui Y. Left ventricular diastolic dysfunction in chronic aortic dissection. Ann Thorac Surg.2007;83(4):1356-60

3. Werner GS, Schaefer C, Dirks R, Figulla HR, Kreuzer H.Prognostic value of Doppler echocardiographic assessment of left ventricular filling in idiopathic dilated cardiomyopathy.Am J Cardiol.1994;73(11):792-8

4. Pinamonti B, Zecchin M, Di Lenarda A, Gregori D, Sinagra G, Camerini F. Persistence of restrictive left ventricular filling pattern in dilated cardiomyopathy: an ominous prognostic sign.J Am Coll Cardiol.1997;29(3):604-12.

5. Whalley GA, Gamble GD, Doughty RN.Restrictive diastolic filling predicts death after acute myocardial infarction: systematic review and meta-analysis of prospective studies.Heart.2006 Nov;92(11):1588-94.

6. Quintana M, Edner M, Kahan T, Hjemdahl P, Sollevi A, Rehnqvist N.Is left ventricular diastolic function an independent marker of prognosis after acute myocardial infarction?Int J Cardiol. 2004;96(2):183-9.

7. Thohan V.Prognostic implications of echocardiography in advanced heart failure. Curr Opin Cardiol. 2004 May;19(3):238-49.

8. Samad BA, Olson JM, Alam M.Characteristics of left ventricular diastolic funcrion in patients with systolic heart failure: a Doppler tissue imaging study.J Am Soc Echocardiogr.2005 Sep;18(9):896-900

9. Liu J, Tanaka N, Murata $\mathrm{K}$, Ueda $\mathrm{K}$ et al..Prognostic value of pseudonormal and restrictive filling patterns on left ventricular remodeling and cardiac events after coronary artery bypass grafting. Am J Cardiol.2003;91(5):550-

10. Bach DS, Bolling SF. Improvement following correction of secondary mitral regurgitation in end-stage cardiomyoparhy with mitral annuloplasty. Am J Cardiol. 1996; 78: 966-69.

11. Tibayan FA, Rodriguez F, Langer $F$ et al.. Undersized mitral annuloplasty alters left ventricular shape during acute ischemic mitral regurgitation. Circulation 2004; 110 [suppl.II]: II 98-II102.

12. McGee EC, Gillinov AM, Blackstone EH et al.Recurrent mitral regurgitation after annuloplasty for functional ischemic mitral regurgitation. J Thorac Cardiovasc Surg 2004, 128:916-24. 
13. Hung J, Papakostas L, Tahta SA et al.Mechanism of recurrent ischemic mitral regurgitation after annuloplasty. Continued LV remodeling as a moving target. Circulation 2004;110[suppl.II]:II 85-II 90.

14. Kuwahara E, Orsuji $Y$, Iguro $Y$ et al.Mechanism of recurrent/persistent ischemic/functional mitral regurgitation in the chronic phase after surgical annuloplasty: importance of augmented posterior leaflet tethering. Circulation 2006, 114(Suppl I): I-529-I 534.

15. Geidel S, Lass M, Schneider C, et al Donwsizing of mitral valve and coronary revascularization in severe ischemic mitral regurgitation results in reverse left ventricular and left atrial remodeling. Eur J Cardiothorac Surg 2005;27:1011-16.

16. Temporelli PL, Giannuzzi P, Nicolosi GL et al.Doppler-Derived Mitral Deceleration Time as a Strong Prognostic Marker of Left Ventricular Remodeling and Survival After Acure Myocardial Infarction Results of the GISSI-3 Echo Substudy. J Am Coll Cardiol 2004:43:1646 -53.

17. Pfeffer MA, Braunwald E, Moye LA et al.Effect of captopril on mortality and morbidity in patients with left ventricular dysfunction after myocardial infarction. Results of the survival and ventricular enlargement trial. The SAVE investigators. N Engl J Med 1992;327:669-77.

18. Carpentier A. Cardiac valve surgery-The "French correction".J Thorac Cardiovasc. Surg. 1983;86(3): 326-37.

19. Schiller NB, Shah PM, Crawford $M$ et al..Recommendations for quantitation of the left ventricle by two-dimensional echocardiography. American Society of Echocardiography Committee on Standards, Subcommittee on Quantitation of Two-Dimensional Echocardiograms.J Am Soc Echocardiogr. 1989;2(5):358-67

20. Kono T, Sabbah HN, Rosman H, Alam M, Jafri S, Goldstein S. Left ventricular shape is primary determinant of functional mitral regurgitation in heart failure. J Am Coll Cardiol 1992;20:159498.

21. Cerqueira MD, Weissman NJ, Dilsizian V et al.American Heart Association Writing Group on Myocardial Segmentarion and Registration for Cardiac Imaging. Standardized myocardial segmentation and nomenclature for tomographic imaging of the heart. A Statement for Healthcare Professionals From the Cardiac Imaging Committee of the Council on Clinical Cardiology of the American Heart Associarion. Circulation 2002;105; 539-42.

22. Gardin JM, Adams DB, Douglas PS et al.American Society of Echocardiography. Recommendations for a standardized report for adult transthoracic echocardiography: a report from the American Society of Echocardiography's Nomenclature and Standards Committee and Task Force for a Standardized Echocardiography Report.J Am Soc Echocardiogr 2002;15 (3):275-90.

23. Enriquez-Sarano M, Tribouilloy C. Quantitation of mitral regurgitation: rationale approach and interpretation in clinical practice. Heart 2002, 88 (suppl IV):1-4.

24. Garcia MJ, Thomas JD, Klein AL. New Doppler echocardiographic applications for the study of diastolic function. J Am Coll Cardiol 1998; 32: 865-75.

25. Eagle KA, Guyton RA, Davidoff $R$ et al. ACC/AHA guidelines for coronary artery bypass graft surgery: executive summary and recommendations: A report of the American College of Cardiology/American Heart Association Task Force on Practice Guidelines (Committee to revise the 1991 guidelines for coronary artery bypass graft surgery)Circulation. 1999;100(13):1464-80. 
26. Ischemic mitral regurgitation: long-term outcome and prognostic implications with quantitative Doppler assessment. Circulation.2001;103(13):1759-64.

27. Calafiore AM, Di Mauro M, Gallina S, Di Giammarco G, Iacò AL, Teodori G, Tavarozzi I.Mitral valve surgery for chronic ischemic mitral regurgitation.Ann Thorac Surg. 2004;77(6):1989-97.

28. Lancellotti P, Lebrun F, Pierard LA. Determinants of exercise-induced changes in mitral regurgitation in patientswith coronary artery diseasc and left ventricular dysfunction.J Am Coll Cardiol. 2003;42(11):1921-8.

29. Byrne JG, Aklog L, Adams DH. Assessment and managementof funcrional or ischaemic mitral regurgitation. Lancet.2000;355:1743-4.

30. Moller JE, Pellikka PA, Hillis GS, Oh JK. Prognostic importance of diastolic function and filling pressure in patients with acute myocardial infarction. Circulation. 2006;114(5):438-44.

31. Rakowski $\mathrm{H}$, Appleton $\mathrm{C}$, Chan $\mathrm{KL}$, et al. Canadian consensus recommendations for the measurement and reporting of diastolic dysfunction by echocardiography: from the Investigators of Consensus on Diastolic Dysfunction by Echocardiography. 1: J Am Soc Echocardiogr. 1996;9(5):736-60.

32. Stellbrink C, Breithardt OA, Franke A ct al. J; PATH-CHF (Pacing Therapies in Congestive Heart Failure) Investigators; CPI Guidant Congestive Heart Failure Research Group. Impact of cardiac resynchronization therapy using hemodynamically optimized pacing on left ventricular remodeling in patients with congestive heart failure and ventricular conduction disturbances.J Am Coll Cardiol. 2001;38(7):1957-65.

33. Romesburg HC. Cluster analysis for researchers. North Carolina: Lulu Press; 2004.1-330.

34. Harrel FE., Lee KL, Califf RM, Pryor DB, Rosati RA. Regression modeling strategies for improved prognostic prediction. Statistics in Medicine 1984; 3: 143-52.

35. Harrel FE Jr, Lee KL, Mark DB. Multivariable Prognostic Models: Issues in developing models, evaluating assumptions and adequacy, and measuring and reducing errors. Stats Med 1996:36187.

36. Hosmer DW, Lemeshow S. Applied Logistic regression.2nd ed.New York, NY: Wiley-Interscience; 2000:143-202.

37. Ereminiene E, Vaskelyte J, Benetis R, Stoskute N. Ischemic mitral valve repair: predictive significance of restrictive left ventricular diastolic filling. Echocardiography. 2005;22(3):217-24.

38. Giannuzzi P, Imparato A, Temporelli PL, et al. Doppler-derived mitral deceleration time of early filling as a strong predictor of pulmonary capillary wedge pressure in postinfarction patients with left ventricular systolic dysfunction. J Am Coll Cardiol 1994;23:1630-7.

39. Appleton CP, Hatle LK, Popp RL. Relation of transmitral velocity patterns to left ventricular diastolic function: new insights from a combined hemodynamic and Doppler echocardiographic study. J Am Coll Cardiol 1988;12:426-40.

40. Cerisano G, Bolognese L, Buonamici P, et al. Prognostic implications of restrictive left ventricular filling in reperfused anterior acute myocardial infarction. J Am Coll Cardiol 2001;37:793-9.

41. Braun J, Bax JJ, Versteegh $\mathrm{MI}$ et al. Preoperative left ventricular dimensions predict reverse remodeling following restrictive mitral annuloplasty in ischemic mitral regurgitation.Eur J Cardiothorac Surg 2005;27(5):847-53. 
180 42. Karagiannis $S \mathrm{E}$, Karatasakis G T, Koutsogiannis N, Athanasopoulos G D, Cokkinos D V. Increased distance between mitral valve coaptation pointand mitral annular plane: significance and correlations in patients with heart failure. Heart 2003;89:1174-1178.

43. Dujardin KS, Enriquez-Sarano M, Rossi A, Bailey KR, Seward JB. Echocardiographic assessment of left ventricular remodeling: are left ventricular diameters suitable tools? J Am Coll Cardiol. 1997 Nov 15;30(6):1534-41.

44. Adams DH, Anyanwu AC. Pitfalls and limitations in measuring and interpreting the outcomes of mitral valve repair. J.Thorac Cardivasc Surg 2006; 131:523-9.

45. Borger MA, Alam A, Murphy PM, Doenst T, David TE. Chronic Ischemic Mitral Regurgitation: Repair Replòace o Rethink? Ann Thorac Surg 2006; 81: 1153-61.

46. Gillinov AM, Wierup PN, Blackstone EH, et al. Is repair preferable to replacement for ischemic mitral regurgitation? J Thorac Cardiovasc Surg. 2001;122(6):1125-41, 


\section{9}

\section{8}

Conclusions

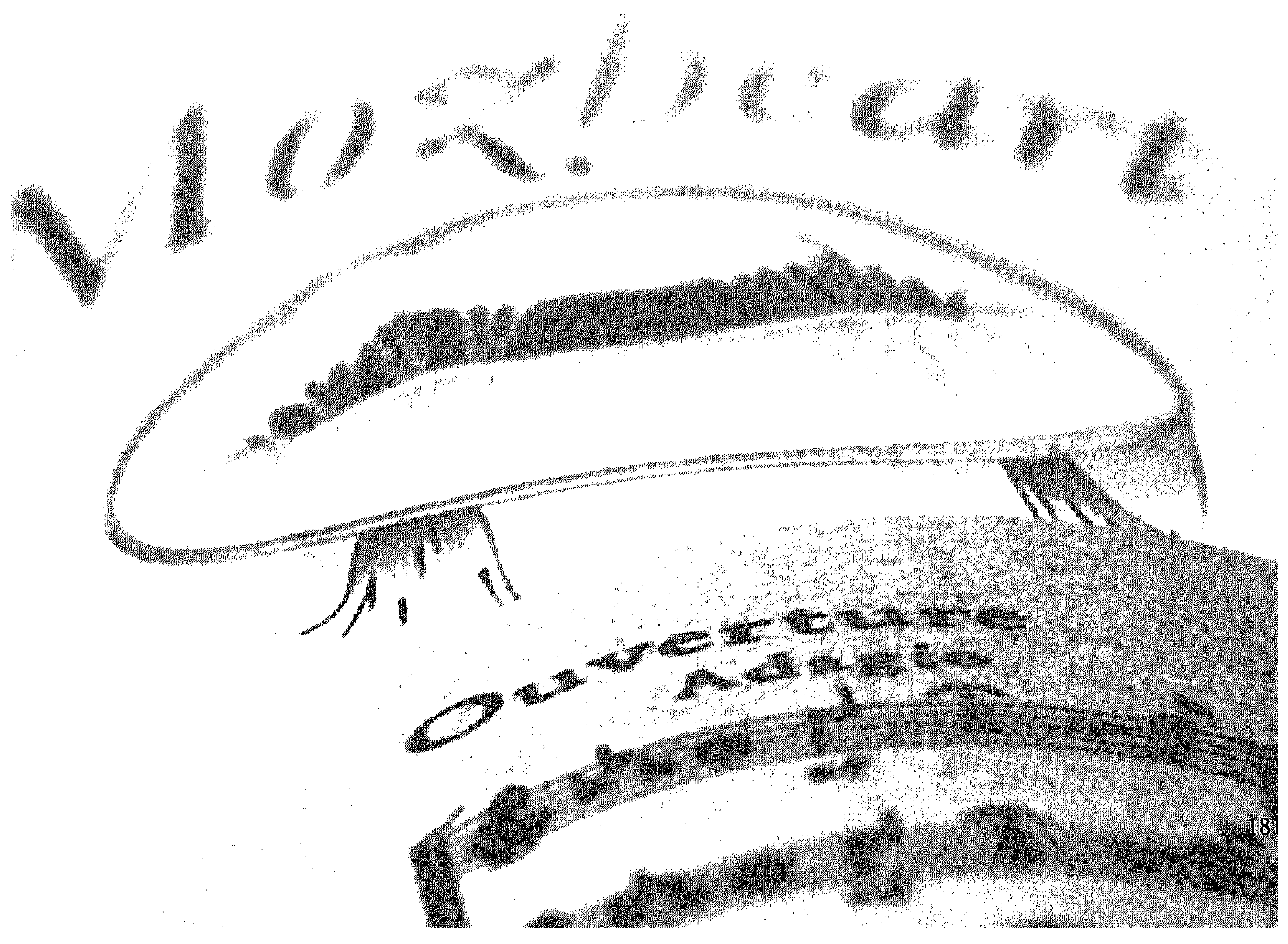


Ischemic mitral regurgitation is mitral regurgitation due to complications of coronary artery disease, in particular, myocardial infarction, and not the fortuitous association of coronary artery disease with intrinsic valve disease such as rheumatic disease. In the acute phase of MI, IMR is frequent and appears to carry an adverse prognosis $^{1-3}$. However, the prognostic implications of IMR in the chronic post-MI phase are uncertain. In pioneering series that underscored the potential importance of IMR, patients were often included early after $\mathrm{MI}^{4}$, and decreased survival of patients with IMR may have been due to inclusion of acute $\mathrm{MI}^{2,3}$. Furthermore, MR angiographic grade was not independently predictive of survival, and only a score combining MR grade with clinical data was a weak independent predictor of Outcome $e^{4}$. Therefore, the SAVE (Survival And Ventricular Enlargement) study data proved of major interest by suggesting that mild IMR was associated with high mortality ${ }^{5}$. However, because the study design excluded MR grade 3 or 4 and limited inclusion to 16 days after $\mathrm{MI}$, the prognostic implications of IMR remain uncertain, particularly regarding specific implications of the full range of chronic IMR. Nevertheless, these pioneering series had the undisputed merit of raising the hypothesis that IMR, which affects $19 \%$ of patients after $\mathrm{MI}^{4,5}$, may be a marker of poor outcome, suggesting that if observed in pure, chronic, definite IMR of all degrees, such an observation may have major prognostic and therapeutic implications.

Chronic Ischemic Mitral Regurgitation (CIMR) which can be due to one of the following reasons: (1) simple annular dilatation (secondary to left ventricular [LV] enlargement), which causes incomplete mitral leaflet coaptation associated with Carpentier type I (normal) leaflet motion; (2) local LV remodeling with papillary muscle displacement producing apical tethering or tenting of the leaflets (with Carpentier type III-b restricted systolic leaflet motion); or (3) both mechanisms ${ }^{6}$. Importantly, exhaustive analyses from the Mayo Clinic echocardiography laboratory ${ }^{7,8}$ have provided a great amount of insight into the mechanisms of ischemic mitral regurgitation and its clinical impact. Thanks to these investigators, we can now reliably measure leaflet tenting area and tenting height quantitatively from transthoracic echocardiograms in patients with LV dysfunction and CIMR, as well as estimate the effective regurgitant orifice?. Larger effective regurgitant orifice, or more leak, was directly and independently predicted by excess leaflet tenting and loss of systolic annular contraction, but it was not related to the degree of LV dysfunction. Adverse prognostic consequences were also clearly documented, moreover, as medium-term survival for patients with CIMR and LV dysfunction was strongly and independently related to calculated effective regurgitant orifice, even after statistical adjustment for all other variables ${ }^{8}$. Recently, Otsuji and associates ${ }^{9}$, experimenting with short-term canine and long-term sheep models and 3-dimensional echocardiographic imaging, also contributed new information concerning the mechanisms of IMR resulting from LV local remodeling. Now that we better understand what we 
are talking about (and can actually measure it!), surgeons should be more aggressive and not ignore substantial degrees of IMR at the time of CABG. This policy should also apply to patients undergoing percutaneous coronary revascularization with percutaneous transluminal coronary angioplasty and stenting to optimize their prognosis, but such is unlikely since we do not live in a utopia and the interventional cardiologists have not figured out quite yet how to repair leaking mitral valves in the cardiac catheterization laboratory.

Although Undersized Ring Annuloplasty associated to CABG is effective in the treatment of CIMR ${ }^{10-12}$, a considerable number of patients show recurrent/persistent $\mathrm{MR}^{13-15}$, which adversely affects mortality ${ }^{16}$ without established mechanism. Mitral leaflet tethering by the outward displacement of papillary muscles caused by $\mathrm{LV}$ remodeling is the basic mechanism of ischemic $\mathrm{MR}^{8,17-22}$. Surgical annuloplasty, which may hoist the posterior annulus anteriorly without displacing the anterior annulus fixed at the aortic root, can potentially and specifically augment posterior leaflet (PML) tethering by increasing the distance between the PM tip and posterior annulus without affecting anterior leaflet (AML) tethering ${ }^{23}$.

Thus, is UMRA reliable and effective, or better, is it effective for all CIMR patients? Does one do a ring annuloplasty or a more reliable chordal-preserving Mitral valve replacement? The answer is not a simple one. Historically, we have been handicapped by comparing very different cohorts of patients in attempting to assess whether repair offers a better outcome than does MVR. The patients receiving these two procedures were very dissimilar and not directly comparable, truly an apples versus oranges situation. Fallacious conclusions resulted from such comparisons.

Gillinov et al. analyzed 482 patients $^{24}$ using propensity score analysis and other sophisticated statistical methods to generate quintiles of patients undergoing either repair or MVR that were relatively well matched to answer the question whether one procedure is better than the other, and, if so, in which particular patient. This approach identified subsets of patients (unbalanced in numbers) that were reasonably well balanced in terms of risk factors. Bootstrap resampling (so-called "bagging") was also used to validate the results of the model and confirm that the results were generalizable. About $70 \%$ of patients -but not the sickest patients- were believed to benefit from repair. The amount of relative benefit of repair in terms of survival was less or actually erased if a thoracic artery was not used for CABG, a lateral LV wall motion abnormality was present, or the IMR jet was "complex." The sickest patients did equally poorly with either procedure, and in certain cases MVR was in fact associated with better predicted survival. Overall 5-year survival was still disappointing, regardless of whether the valve was repaired or replaced.

Grossi and associates ${ }^{25}$ evaluated 223 patients with IMR; but, the New York University statisticians concluded that the marked imbalance in numbers of patients undergoing one procedure or another within the quintiles was such that the propensity score models did not provide an adequate fit to the data. Therefore, propen- 
184 sity score adjustments were not used. Instead, the New York University group did multiple convoluted layers of multivariable analyses to adjust for the confounding effects of functional disability (New York Heart Association class), presence of angina, and particular operation performed, followed by separate Cox modeling within each of the two surgical groups. Those patients who underwent mitral repair at New York University were not as sick as those who required MVR (as was also the case in the Gillinov report ${ }^{24}$ ); medium-term survival was suboptimal but roughly equivalent between the two types of procedures. Certain subsets of patients were identified who appeared to do better if they could undergo repair.

Therefore, even with methodological pitfalls ${ }^{26}$ these articles show a superiority of mitral valve repair over replacement. Valve repair with an undersized annuloplasty ring works satisfactory in most cases of CIMR, but the surgeon needs to pay keen attention in interpreting clinical and echocardiographic data.

The aims of this thesis were: 1) to report long-term results of combined reductive annuloplasty and coronary artery bypass grafting in terms of recurrence of mitral regurgitation, left ventricular reverse remodeling, continued left ventricular remodeling, left ventricular dimensions and geometry. 2) to study modifications of LV and valvular conformation and geometry in chronic ischemic mitral regurgitation before and after annuloplasty. 3) to identify mechanisms (either ventricular or valvular) of recurrent mitral regurgitation 4) to recognize preoperative factors who can help to select patients who can really benefit from annuloplasty.

One of the main limitations of previous clinical studies on chronic IMR is the lack of a clear definition. Different descriptions have resulted in heterogeneous patient groups, which in turn complicate comparisons between studies. In the studies reported in this thesis care was taken in the selection of patients and clear definitions were adopted to ensure homogeneity of patient populations and facilitate comparisons between studies.

\section{TRUE DEFINITION OF CIMR}

Serri and colleagues ${ }^{27}$ defined ischemic mitral regurgitation as "mitral regurgitation resulting from prior myocardial infarction associated with normal mitral valve leaflets and chordae." This is an etiologic definition that fails to take into account the valve dysfunction or the lesions that actually result in the mitral regurgitation. What is a "normal mitral leaflet"? Carpentier's pathophysiologic triad describes the interrelationship between leaflet motion (dysfunction), lesions, and etiology ${ }^{6}$. Carpentier's classification of dysfunction is based on the opening and closing motions of the mitral leaflets in relation to the annular plane. Most patients with ischemic mitral regurgitation have type IIIb leaflet dysfunction, with restricted leaflet motion in systole. Wall motion abnormalities, associated with left ventricular dilatation and lateral or apical papillary muscle displacement, result in this type 
of valve dysfunction. The lesion associated with type IIIb dysfunction is primarily leaflet tethering, especially the posterior-medial scallop of the posterior leaflet (P3). Associated annular dilatation may be present, but leaflet tethering is considered the primary lesion. Type I dysfunction (normal leaflet motion) with isolated annular dilatation is less common but may occur in the setting of basal myocardial infarction. Some patients with ischemic mitral regurgitation have type II (excess leaflet motion) dysfunction. The lesions resulting in type II ischemic mitral regurgitation include an acutely ruptured papillary muscle or a previously infarcted fibrotic and elongated papillary muscle. In rare instances, isolated chordal rupture can also be seen following papillary tip ischemia.

We suggest a more uniform methodology to classify mitral valve cohorts not only on the basis of etiology but inclusive of dysfunctions and lesions. The importance of such an approach is well demonstrated in ischemic mitral regurgitation. The majority of patients have type IIIb dysfunction, and a remodeling annuloplasty must be undersized to overcorrect the septal-lateral dimension and achieve an adequate surface of leaflet coaptation. Patients with ischemic mitral regurgitation and leaflet restriction (type IIIb dysfunction) are more likely to have residual leaks compared with patients with type I dysfunction who have normal leaflet motion and are treated in a similar fashion. Furthermore, patients with ischemic mitral regurgitation and type II dysfunction have a better long-term prognosis than those with type IIIb dysfunction ${ }^{24}$. Thus it is inadequate to describe a population of patients with ischemic mitral regurgitation solely on the basis of etiology. This point is equally valid in patients with a "degenerative" etiology of mitral valve disease. Prolapse (type II dysfunction) of the posterior leaflet due to a single ruptured chord (isolated lesion) has a markedly different repair rate and long-term freedom from recurrent mitral regurgitation compared with bileaflet prolapse with chordal rupture and/or elongation involving multiple leaflet segments. Definition of a population of mitral valve patients on the basis of etiology, dysfunction, and lesions is necessary to allow adequate comparison of outcomes, as all can impact clinical results.

\section{WHICH RING?}

To extrapolate the efficacy of a surgical technique, it is important that the reader determines whether the technique under evaluation was applied in a standardized and systematic manner that upholds current principles and understanding of pathophysiology. This is particularly relevant in mitral valve repair surgery, as the quality of the result judged by residual or recurrent mitral regurgitation is largely dependent on the manner in which the repair has been performed. Indeed valve sizing should be undertaken in a standardized and logical fashion to define the current efficacy of ischemic mitral valve repair. Because it is now widely accepted that use of an appropriately downsized ring is the single most important aspect 
of mitral repair for ischemic mitral regurgitation, the absence of a precise sizing technique raises doubts regarding surgical approach and could explain suboptimal long-term results in some studies ${ }^{27}$.

The use of incomplete flexible posterior annular bands further complicates interpretation of results ${ }^{27}$. Recent articles have linked flexible posterior bands with significant recurrent ischemic mitral regurgitation ${ }^{13.14}$. It is now well established that patients with ischemic mitral regurgitation can develop dilatation of the anterior annulus between the trigones ${ }^{28,29}$. Because posterior flexible bands do not correct inter trigonal dilatation, they may not effectively ensure restoration of an adequate surface of leaflet coaptation.

In our studies we undertook valve sizing in a standardized fashion and the degree of undersizing was homogeneous over the 5-year-period of the study. Finally, we used only two rings (Carpentier's rigid or semi-flexible rings). Even these two rings are not at all identical, thus, if we have undersized by two sizes both rings, we might have been less restrictive with the Physio ring than with the Classic ring. However, in our experience, neither ring type nor ring size were predictive of recurrent MR. Recently, we have employed new rings which mimic the shape of healthy mitral annulus, and, in contrast with rigid/semi-flexible plane annuloplasty rings, do not flatten the natural saddle shape causing greater tension on chordae and leaflets, innovative annular rings mimic the shape of healthy mitral annulus ${ }^{30}$.

\section{Persistent (Residual) vs. Recurrent Mitral Regurgitation}

Significant persistent (residual) mitral regurgitation following valve repair, documented either on postbypass transesophageal echocardiography (TEE) or predischarge transthoracic echocardiography, implies an immediate failure of the technique employed. In such cases the valve repair was really never successful, and it is not surprising that regurgitation persists into the long term. It would be incorrect to label such cases as "recurrent" mitral regurgitation in studies of long-term outcomes, as the mitral regurgitation was never eliminated at surgery. In the same way that recurrent cancer can only be defined when the primary tumor was successfully and completely resected, recurrent mitral regurgitation should be defined only when surgery initially eliminated the mitral regurgitation. These definitions are often not clarified, and residual mitral regurgitation may account for a substantial proportion of "recurrent" mitral regurgitation reported after ischemic mitral valve repairs. For example, Hung and colleagues ${ }^{15}$ studied 30 patients with "recurrent" mitral regurgitation after annuloplasty for ischemic mitral regurgitation in an attempt to understand the mechanisms of recurrence, yet $30 \%$ of these patients had moderate or severe mitral regurgitation on the early postoperative study and should have been categorized with residual mitral regurgitation. In addition Kuwahara et $\mathrm{a}^{31}$ and Magne and coworkers ${ }^{32}$ studied recurrent/persistent and persistent MR, 
respectively, whereas, in our study residual MR was excluded. Indeed, persistent /residual MR is presumably due to immediate failure of the technique employed rather than $L V$ remodeling ${ }^{33}$ and it is not surprising that regurgitation persists at late follow up in these patients. In our opinion, surgeons generally should not accept any degree of mitral regurgitation other than trivial or mild on the postbypass TEE, as the freedom for need of reoperation, reverse ventricular remodeling, and resulting improvements in quality of life are at least in part dependent on elimination of mitral regurgitation. Significant degrees of residual mitral regurgitation (moderate to severe mitral regurgitation and sometimes even mild mitral regurgitation) should generally trigger a second bypass run and corrective action (for example, further ring downsizing, leaflet patch extension, correction of unrecognized prolapse, or valve replacement).This need for a second bypass run should be uncommon and probably not exceed $5 \%$ as mitral regurgitation should be eliminated in most patients with correct ring choice and sizing. Only in exceptional circumstances should patients leave the operating room with residual moderate mitral regurgitation; such as when a valve is deemed irreparable and nonreplacable (e.g., massive atrial/annular calcification) or when the risks of a second bypass run are thought to outweigh the benefits. Any study with a high degree of residual mitral regurgitation may be relied on to define perioperative but not long-term outcomes of mitral valve repair.

\section{WHAT DID WE LEARN?}

In our experience, $72 \%$ of patients undergoing combined CABG and UMRA showed, at five-year control, recurrent MR. In addition, left ventricular reverse remodeling was observed only in $44.2 \%$ of the study population with $10.3 \%$ of patients showing further left ventricular dilatation.

We clearly identified two categories of patients with CIMR referred to surgery: in one Group subjects showed a symmetric tethering pattern and a mainly posterior regurgitant jet. The second Group had a symmetrical tethering with a central jet. Whereas this difference did not reflect, in our experience, a different infarct localization, indeed a different pattern of local remodeling was responsible for this discrepancy: a more posterior local LV remodeling in the first group and an anterior-posterior LV remodeling with anterior predominance in the symmetric Group. Patients with higher preoperative AML tethering had a greater local LV remodeling of the segments of the anterior papillary muscle, a greater lateral and posterior displacement of the anterior papillary muscle and a wider $\mathrm{PM}_{\mathrm{S}}$ separation and a more symmetric tethering pattern. However patients with postoperative MR showed a mechanism different from that of MR at baseline, with a tethering pattern becoming progressively more asymmetric. This is due to continued remodeling that is mainly posterior in the postoperative period, whereas the LV remodeling in the area 
of the AML and, consequently, the anterior leaflet tethering, is maintained as in the preoperative situation.

These findings support previous clinical results reported by Agricola et $\mathrm{al}^{34}$, who described two Groups of patients with CIMR: likewise patients with more pronounced AML tethering, their symmetric Group had prevalent posterior and lateral displacement of anterior papillary muscle, $\mathrm{PM}_{\mathrm{s}}$ separation and WMSI of the regions supporting the anterior papillary muscle.

Continued LV remodeling occurs in all Groups in the postoperative period and it predominantly involves the region of the LV supporting the posterior papillary muscle. There was no continued anterior remodeling as demonstrated by wall motion score index of segments underlying the anterior papillary muscle which remained constant over time postoperatively in the entire patient cohort. This is in accordance with published data ${ }^{15,35}$ and confirms that tethering of the posterior leaflet may be exacerbated by annuloplasty which, reducing annular size, can potentially shift the posterior annulus farther anteriorly, increasing its displacement relative to the papillary muscles without displacing the anterior annulus fixed at the aortic $\operatorname{root}^{36}$.

Thus our results showed the evidence that preoperative different pattern translated into different postoperative results with most of the symmetric tethering showing recurrent MR. Indeed this finding should be identified keeping in mind that these patients are more likely to develop MR. A key point is the length/ mobility of the anterior mitral leaflet evaluated either at echochardiography or at surgical inspection. We confirmed that an AML longer than A-P diameter is necessary but it could not suffice to ensure durability. The possibility of allowing both leaflets into apposition depends on the ability of AML to move toward the annulus and to reach the posterior one. If this movement is insufficient the mitral leaflets never coapt no matter how much posterior annulus is reduced. The length should allow gaining a postoperative coaptation length of at least $0.8 \mathrm{~cm}$ (better $1 \mathrm{~cm}$ ) which ensures a proper coaptation for long time even in the presence of continued posterior remodeling. The role of the posterior leaflet is not "functional" but it is necessary to offer a "coaptation surface" to the AML, necessary for a proper valve closure. In fact we demonstrated that, even in the presence of a mobile, long AML, a proper continence is not ensured if the AML do not close against the PML.

The angle $\alpha^{\prime}$ gives an accurate measure of AML tethering. Furthermore, the length of the AML can be estimated by the coaptation height which represents the depth of the AML during systole, in our opinion and according to Calafiore et a ${ }^{37}$, when it is more than $11 \mathrm{~mm}$, it is associated with a high risk of MR recurrence after reductive annuloplasty.

Thus patients with symmetric tethering and AML tethering predominance $\left(\alpha^{\prime} / \beta^{\prime} \rightarrow 1\right)$, AML not mobile and more tethered $\left(\alpha \geq 39.5^{\circ}(p<0.001)\right.$, anterior leaflet excursion angle $\leq 35^{\circ}$ ) with a coaptation height $\geq 11 \mathrm{~mm}$ will not benefit from UMRA and these results suggest the need, in these subjects, for different approaches directly 
addressing ventricular tethering or a chordae-sparing mitral valve replacement. To our knowledge our studies provide the first demonstration that MR recurrence can be predicted from the AML tethering pattern of the ischemic mitral valve on the preoperative echocardiographic evaluation. Nonetheless, the influence of AML tethering could be secondary to other factors and the altered leaflet geometry and recurrent MR could represent both the result of abnormal left ventricular function and geometry which may be primary predictors. This is why we carried out a study in which multivariable models were adjusted for important risk factors of MR recurrence. This study demonstrated that, after we accounted for other clinical and echocardiographic key risk factors AML tethering angle remained a strong predictor of recurrent regurgitation. Indeed patients with moderate or moderate-severe preoperative AML tethering showed a 2.5 times and 3 times higher Odds ratio for MR recurrence compared to those with significant PML tethering (PML angle $\geq 45$ degrees $^{31}$ ). This rose to $>4$ fold in patient with severe AML tethering.

In contrast with our results, Kuwahara et $\mathrm{al}^{31}$ showed that increased PML tethering was the primary determinant of late MR in 30 patients undergoing surgical annuloplasty for ischemic mitral regurgitation. In addition, Magne and coworkers $^{32}$ identified a PML angle $\geq 45$ degrees (sensitivity $100 \%$, specificity $97 \%$ ) to be a predictor of postoperative persistence of MR after annuloplasty.

However, these authors studied recurrent/persistent and persistent MR, respectively, whereas in our study residual MR was excluded. Persistent/residual MR is presumably due to immediate failure of the technique employed rather than LV remodeling ${ }^{22}$ and it is not surprising that regurgitation persists at late follow up in these patients

\section{Final Considerations}

When I present my works at meetings/congresses worldwide I have very often to answer questions like this "I am a cardiac surgeon, how can I understand echocardiographic angles, excursions... ? These are of main interest to cardiologists"

My answer is: "I am a cardiac surgeon too but I believe that it is much easier to ask for a couple of echocardiographic indexes rather than to re-plasty or replace an already repaired mitral valve" 


\section{REFERENCES}

1. Barzilai B, Gessler C Jr, Perez JE, et al. Significance of Doppler-detected mitral regurgitation in acute myocardial infarction. Am J Cardiol. 1988;61:220 -223.

2. Lehmann KG, Francis CK, Dodge HT. Mirral regurgitation in early myocardial infarction: incidence, clinical detection, and prognostic implications: TIMI Study Group. Ann Intern Med. 1992;117:10-17.

3. Tcheng JE, Jackman JD Jr, Nelson CL, et al. Outcome of patients sustaining acute ischemic mitral regurgitation during myocardial infarction. Ann Intern Med. 1992;117:18 -24.

4. Hickey MS, Smith LR, Muhlbaier LH, et al. Current prognosis of ischemic mitral regurgitation: implications for future management. Circulation.1988;78:I-51-I-59.

5. Lamas GA, Mitchell GF, Flaker GC, et al. Clinical significance of mitral regurgitation after acute myocardial infarction: Survival And Ventricular Enlargement (SAVE) Investigators. Circulation. 1997;96:827-833.

6. Carpentier A. Cardiac valve surgery-The "French correction." J ThoracCardiovasc Surg. 1983;86:323-37.

7. Grigioni F, Enriquez-Sarano M, Zehr KJ, Bailey KR, Tajik AJ. Ischemic mitral regurgitation: long-term outcome and prognostic implications with quantitative Doppler assessment.Circulation 2001;103(13):1759-64.

8. Yiu SF, Enriquez-Sarano M, Tribouilloy C, Seward JB, Tajik AJ. Determinants of the degree of functional mitral regurgitation in patients with systolic left ventricular dysfunction: a quantitative clinical study. Circulation. 2000;102:1400-1406.

9. Otsuji Y, Handschumacher MD, Liel-Cohen N, Tanabe H, Jiang L, chwammenthal E, et al. Mechanism of ischemic mitral regurgitationwith segmental left ventricular dysfunction: threedimensionalechocardiographic studies in models of acute and chronic progressiveregurgitation. J Am Coll Cardiol. 2001;37:641-8.

10. Bolling SF, Pagani FD, Deeb GM, Bach DS. Intermediate-term outcome of mitral reconstruction in cardiomyopathy. J Thorac Cardiovasc Surg.1998;115:381-386; discussion 387-388.

11. Bax JJ, Braun J, Somer ST, Klautz R, Holman ER, Versteegh MI, Boersma E, Schalij MJ, van der Wall EE, Dion RA. Restrictive annuloplasty and coronary revascularization in ischemic mitral regurgitation results in reverse left ventricular remodeling. Circulation. 2004;110(Suppl II):II103-II108.

12. Calafiore AM, Gallina S, Di Mauro M, Gaeta F, laco AL, D'AlessandroS, Mazzei V, Di Giammarco G. Mitral valve procedure in dilated cardiomyopathy: repair or replacement? Ann Thorac Surg. 2001;71:1146-1152.

13. Tahta SA, Oury JH, Maxwell JM, Hiro SP, Duran CM. Outcome aftermitral valve repair for functional ischemic mitral regurgitation. J HeartValve Dis. 2002;11:11-18.

14. McGee Jr EC, Gillinov AM, Blackstone EH, Rajeswaran J, Cohen G, Najam F, Shiota T, Sabik JF, Lycle BW, McCarthy PM, Cosgrove DM.Recurrent mitral regurgitation after annuloplasty for functional ischemic mitral regurgitation. J Thorac Cardiovasc Surg. 2004;128:916 -924. 


\section{Conclusions}

15. Hung J, Papakostas L, Tahta SA, Hardy BG, Bollen BA, Duran CM, Levine RA. Mechanism of recurrent ischemic mitral regurgitation afterannuloplasty: continued $L V$ remodeling as a moving target. Circulation. 2004;110(Suppl II):II85-II90.

16. Dahlberg PS, Orszulak TA, Mullany CJ, Daly RC, Enriquez-Sarano M, Schaff HV. Late outcome of mitral valve surgery for patients with coronary artery disease. Ann 'Thorac Surg. 2003;76:1539 -1548 .

17. Godley RW, Wann LS, Rogers EW, Feigenbaum $\mathrm{H}$, Weyman AE. Incomplete mitral leaflet closure in patients with papillary muscle dysfunction.Circulation. 1981;63:565-571.

18. Otsuji Y, Handschumacher MD, Schwammenthal E, Jiang L, Song JK, Guerrero JL, Vlahakes GJ, Levine RA. Insights from three-dimensionalechocardiography into the mechanism of functional mitral regurgitation: direct in vivo demonstration of altered leaflet tethering geometry. Circulation.1997;96:1999-2008.

19. Gorman RC, McCaughan JS, Ratcliffe MB, Gupta KB, Streicher JT, Ferrari VA, St John-Sutton MG, Bogen DK, Edmunds LH Jr. Parhogenesisof acute ischemic mitral regurgitation in three dimensions.J Thorac Cardiovasc Surg. 1995;109:684-693.

20. Komeda M, Glasson JR, Bolger AF, Daughters GT 2nd, MacIsaac A, Oesterle SN, Ingels NB Jr., Miller DC. Geometric determinants of ischemicmitral regurgitation. Circulation. 1997;96(Suppl II): II $128-$ III 133.

21. Agricola E, Oppizzi M, Maisano F, De Bonis M, Schinkel AFL, TorraccaL, Margonato A, Melisurgo $G$, Alfieri $O$. Echocardiographic classificationof chronic ischemic mitral regurgitation caused by restricted motion according to tethering pattern. Eur J Echocardiogr. 2004;5:326334.

22. Watanabe N, Ogasawara Y, Yamaura Y, Kawamoto T, Toyota E, Akasaka T, Yoshida K. Quantitation of mitral valve tenting in ischemic mitral regurgitation by transthoracic real-time threedimensional echocardiography.J Am Coll Cardiol. 2005;45:763-769.

23. Green GR, Dagum P, Glasson JR, Nistal JF, Daughters GT 2nd, IngelsNB Jr., Miller DC. Restricted posterior leaflet motion after mitral ring annuloplasty. Ann Thorac Surg. 1999;68:2100 -2106 .

24. Gillinov AM, Wierup PN, Blackstone EH, Bishay ES, Cosgrove DM, White J, et al. Is repair preferable to replacement for ischemic mitral regurgitation? J Thorac Cardiovasc Surg. 2001;122:1125-41.

25. Grossi EA, Goldberg JD, LaPietra A, Ye X, Zakow P, Sussman M, etal. Ischemic mitral valve reconstruction and replacement: comparison of long-term survival and complications. J Thorac Cardiovasc Surg.;122:1107-24.

26. Miller CD. Ischemic Mitral regurgitation.Redux-to repair o to replace. J Thorac Cardiovasc Surg.;122:1059-62.

27. Serri K, Bouchard D, Derners P, Coutu M, Pellerin M, Carrier M, etal. Is good perioperative echocardiographic result predictive of durabilityin ischemic mitral valve repair. J Thorac Cardiovasc Surg. 2006;131:565-73. 
28. Heub AC, Jatene FB, Moreira LF, Pomerantzeff PM, Kallas E, deOliveira SA. Ventricular remodeling and mitral valve modifications indilated cardiomyopathy: new insights from anatomic study. J Thorac Cardiovasc Surg. 2002;124:1216-24.

29. Ahmad RM, Gillinov AM, McCarthy PM, Blackstone EH, Apperson-Hansen C, Qin JX, et al. Annular geometry and motion in humanischemic mitral regurgitation: novel assessment with three-dimensional echocardiography and computer reconstruction. Ann Thorac Surg. 2004;78:2063-8.

30. Votta E, Maisano F, Bolling SF, Alfieri O, Montevecchi FM, Redaelli A. The Geoform diseasespecific annuloplasty system: a finite element study. Ann Thorac Surg. 2007;84(1):92-101.

31. Kuwahara E, Otsuji $Y$, Iguro $Y$ et al. Mechanism of recurrent/ persistent ischemic/functional mitral regurgitation in the chronic phase after surgical annuloplasty: importance of augmented posterior leaflet tethering. Circulation 2006;114(Suppl.):I529-I534.

32. Magne J, Pibarot P, Dagenais F, Hachicha Z, Dumesnil JG, Senechal M. Preoperative posterior leaflet angle accurately predicts outcome after restrictive mitral valve annuloplasty for ischemic mitral regurgitation.Circulation. 2007;115(6):782-91.

33. Adams DH, Anyanwu A. Pitfalls and limitations in measuring and interpreting the outcomes of mitral valve repair. J Thorac Cardiovasc Surg. 2006; 131(3):523-9.

34. Agricola E, Oppizzi M, Maisano $F$ et al. Echocardiographic classification of chronic mitral regurgitation caused by restricted motion according to tethering pattern. Eur JEchocardiography 2004;5:326-34.

35. Zhu F, Otsuji Y, Yotsumoto G, et al. Mechanism of persistent ischemic mitral regurgitation after annuloplasty: importance of augmented posterior mitral leaflet tethering.Circulation. 2005;112(Suppl):[396-401.

36. Green GR, Dagum P, Glasson JR, et al. Restricted posterior leaflet motion after mitral ring annuloplasty.Ann Thorac Surg1999;68(6):2100-6.

37. Calafiore AM, Di Mauro $M$, Gallina $S$, et al. Mitral valve surgery for chronic ischemic mitral regurgitation.Ann Thorac Surg.2004;77(6):1989-97. 

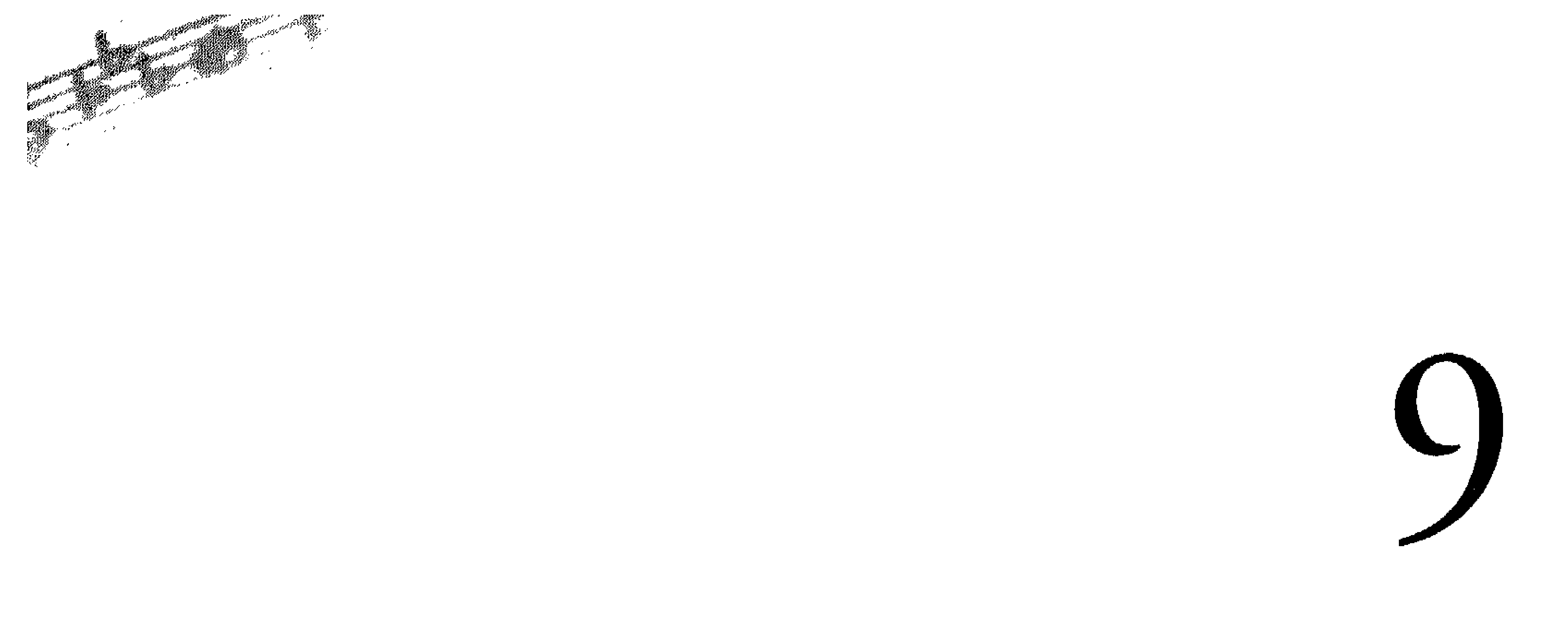

Summary

Riassunto

Samenvatting

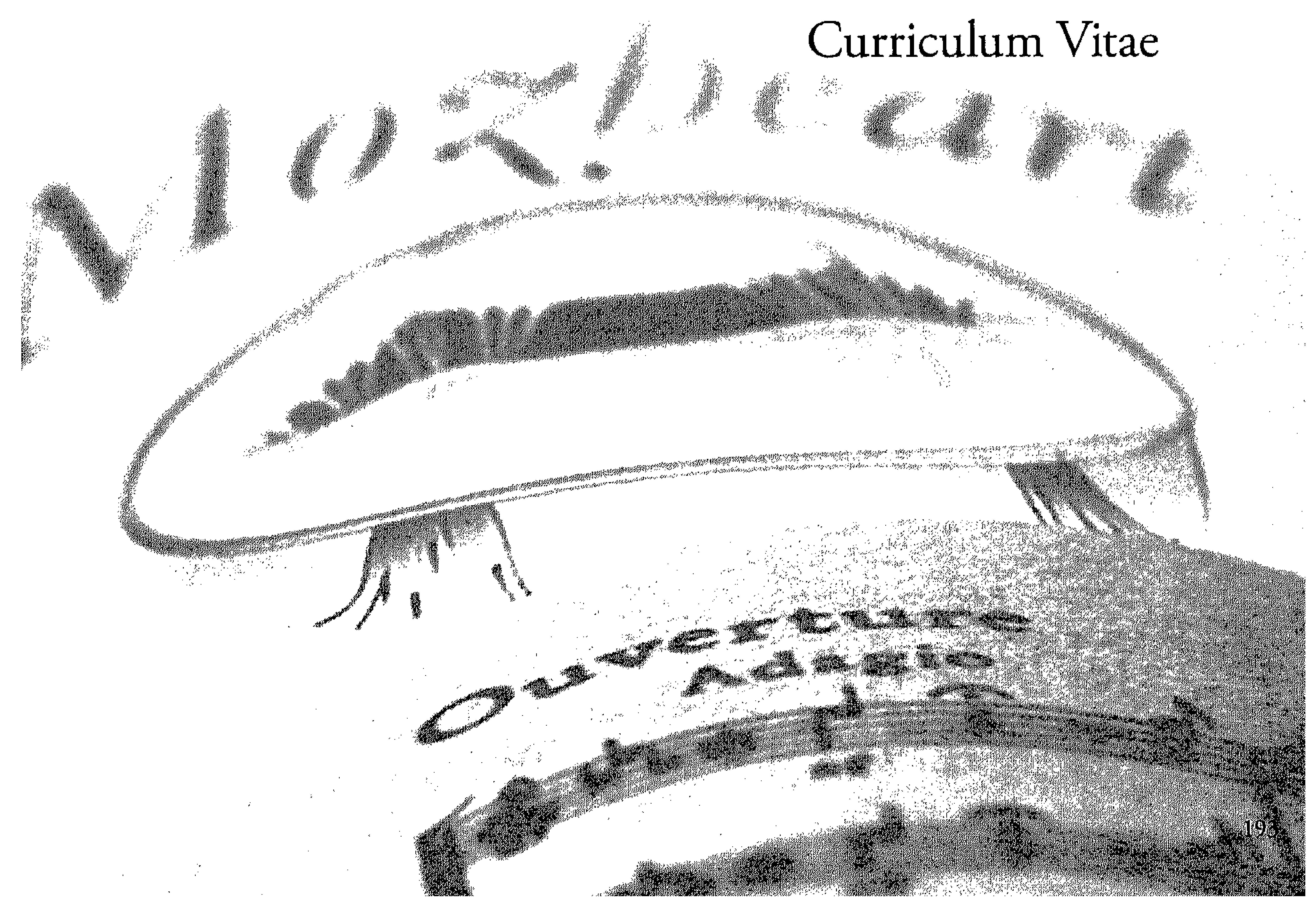


Summary 
The aims of this thesis were: 1) to report long-term results of combined reductive annuloplasty and coronary artery bypass grafting in patients with chronic ischemic mitral regurgitation, in terms of recurrence of mitral regurgitation, left ventricular reverse remodelling, continued left ventricular remodelling, left ventricular dimensions and geometry 2) to study modifications of LV and valvular conformation and geometry in chronic ischemic mitral regurgitation before and after annuloplasty 3 ) to identify mechanisms (either ventricular or valvular) of recurrent mitral regurgitation 4) to recognize preoperative factors who can help to select patients who can really benefit from annuloplasty.

In Chapter 2 we present 5-year echocardiographic results of combined undersizing mitral ring annuloplasty and coronary artery bypass grafting in ischemic mitral regurgitation. Two-hundred-fifty-one patients were prospectively enrolled in the study. Serial echocardiograms were performed in 220 survivors at baseline, discharge and annually thereafter. MR remained stable at 1 year and re-increased at 3 years and 5 years. Five-year actuarial survival was $83.2 \pm 4.4 \%$. Five-year freedom from reoperation for failed repair was $78.2 \pm 4.9 \%$. Mean systolic and diastolic diameters decreased significantly at discharge and at early follow up but raised at 3 years and 5 years. Systolic and diastolic sphericity indexes improved at discharge, remained stable at 1-year but they re-increased at 3-year control with a late raise exceeding the preoperative value. Left ventricular reverse remodeling was observed in $44.2 \%$ of the study population with $10.3 \%$ of patients showing further left ventricular dilatation. At multivariable model, end-systolic volume $\geq 145 \mathrm{ml}$, systolic sphericity Index $\geq 0.7$, Myocardial performance Index $\geq 0.9$ and wall motion score Index $\geq 1.5$ were predictors of recurrent MR. Our findings emphasize the need for improved repair technique and better patient selection to identify patients with anticipated repair failure who could benefit more from valve replacement or other procedure directly addressing ventricular tethering.

In Chapter 3 We analyzed left ventricular reverse remodeling after combined coronary artery bypass grafting and undersized mitral ring annuloplasty examining the difference in the benefit of annuloplasty on clinical end points and recurrence of mitral regurgitation between responders and non-responders of left ventricular reverse remodeling. Study eligibility criteria were fulfilled by 204 patients with chronic ischemic mitral regurgitation who survived combined coronary artery bypass and reductive annuloplasty. Reverse remodeling was defined as a reduction in left ventricular end systolic volume Index $>15 \%$. There were $84(41.2 \%)$ responders of reverse remodeling and $120(58.8 \%)$ non-responders. Non-responders had a higher recurrence of mitral regurgitation, higher reoperation rate for failed repair significantly larger left ventricular volumes and dimension at any study point with significant late increase of sphericity indexes exceeding preoperative values. At multivariable analysis baseline myocardial performance Index $<0.90$, systolic sphericity Index $<0.72$ and wall motion score Index $<1.59$ resulted as independent predictors of 
reverse remodeling. Our experience suggests that more information on possible echo predictors of an inadequate result may improve preoperative decision-making.

Chapter 4 investigates leaflet and subvalvular configurations of the mitral valve to identify mechanisms leading to recurrent mitral regurgitation after combined undersized mitral annuloplasty and coronary artery bypass. The study population consisted of 230 patients who were divided in two Groups: patients without (Group1, $\mathrm{n}=176$ ) or with (Group 2, n=54) late recurrent mitral regurgitation. Fifty normal healthy subjects were controls. Subjects with late regurgitation had preoperatively a more symmetric tethering, a more accentuated anterior mitral leaflet tethering and a more restricted anterior leaflet excursion than Group 1. Postoperatively, tethering of posterior leaflet increased and it was predominant in both groups whereas the tethering of the anterior leaflet reduced at discharge remaining constant afterwards. Multivariable analysis showed an anterior tethering angle $\geq 39.5^{\circ}$, an anterior/posterior tethering angle ratio $\geq 0.76$, an anterior leaflet excursion angle $\leq 35^{\circ}$ and a coaptation height $\geq 11 \mathrm{~mm}$ to be predictors of recurrent mitral regurgitation. Our results demonstrate that preoperative symmetric tethering with anterior mitral leaflet predominance is strongly associated with recurrence of mitral insufficiency. Measures of leaflet tethering results in fundamental findings to identify ischemic patients who can really benefit from restrictive annuloplasty.

In Chapter 5 we test the hypothesis that a specific leaflet configuration with anterior mitral leaflet tethering predominance is related to higher postoperative MR recurrence independently of left ventricular size and geometry. In 278 patients with surgical annuloplasty for chronic ischemic mitral regurgitation and 50 matched normal subjects, posterior and lateral displacement of papillary muscles the anterior leaflet and posterior leaflet tethering angles and their ratio, the coaptation length and the MR grade were quantified before and at follow up. Patients were divided in 5 Groups on the basis of the preoperative anterior mitral leaflet tethering angle: Group 1 (normal/slight tethering, n=33), Group 2, (mild tethering, n=55), Group 3 (moderate tethering, $n=63$ ), Group 4 (moderate-severe tethering, $n=64$ ) and Group 5 (severe tethering, $n=63$ ). Subjects with anterior mitral leaflet tethering $\geq$ moderatesevere had preoperatively a more symmetric tethering, a more accentuated anterior papillary muscle displacement and a larger anterior local remodeling. Recurrence of MR was significantly higher in patients with moderate-severe/severe anterior mitral leaflet tethering. Postoperatively, tethering of posterior leaflet increased and it was predominant without difference between groups, resulting in a more asymmetric tethering in the major part of patients with recurrent regurgitation. At multivariable logistic regression the anterior leaflet tethering angle was the primary determinant of regurgitation recurrence, even after adjustment of known risk factors. Our results confirm that the grade of preoperative anterior mitral leaflet tethering is strongly associated with recurrence of ischemic regurgitation. 
The predictive value of diastolic function on clinical outcome and recurrence of ischemic mitral regurgitation following combined undersized mitral annuloplasty and coronary artery bypass grafting is reported in Chapter 6 . Two hundred-thirtyfour patients with chronic ishemic mitral regurgitation who survived combined restrictive annuloplasty and coronary bypass, were divided into four groups on the basis of baseline Deceleration Time and systolic-diastolic pulmonary venous flow ratio: Group 1, normal $(n=48)$, Group 2, impaired relaxation $(n=61)$, Group 3 , pseudonormal pattern $(n=60)$ and Group 4, restrictive pattern $(n=65)$. Early mortality rate was highest in the restrictive group (9.2\%). In addition 6-year actuarial survival was significantly lower in Group 4. Furthermore, a deceleration time $<140 \mathrm{~ms}$ and a systolic/diastolic pulmonary venous flow $<0.80$ were independent predictors of early and late death. Finally, a deceleration time $<140 \mathrm{~ms}$ was the only diastolic independent predictor of recurrence of ischemic regurgitation. Restrictive left ventricular diastolic filling pattern is an important preoperative marker of high early and late death and recurrence of regurgitation in patients undergoing reductive annuloplasty for ischemic mitral regurgitation. In our experience left ventricular diastolic filling pattern is an important preoperative marker of high early and late death and recurrence of regurgitation.

In Chapter 7 we explored the predictive value of Doppler-Derived Mitral Deceleration Time on left ventricular reverse remodeling in patients with chronic ischemic mitral regurgitation undergoing combined undersized mitral annuloplasty and coronary artery bypass grafting. Two hundred and fifteen patients undergoing combined undersized annuloplasty and coronary artery bypass were divided into four groups on the basis of baseline deceleration time: Group 1 (normal, $n=48$ ), Group 2 (impaired relaxation, $n=61$ ), Group 3 (pseudonormal pattern, $n=50$ ) and Group 4 (restrictive pattern, $\mathrm{n}=56$ ). Left ventricular reverse remodeling, defined as a reduction in end systolic volume Index $>15 \%$, occurred in $95.7 \%, 96.3 \%, 88.3 \%$ and $0 \%$ in Groups 1,2,3 and 4, respectively. Logistic regression analysis showed that a deceleration time $\leq 125 \mathrm{~ms}$ was a strong predictor of reverse remodeling after annuloplasty. Preoperative assessment of this parameter adds significant information to commonly used indexes of global and regional function, and represents a very easy and cost effective tool to accurately identify those patients who can really benefit from annuloplasty.

We can conclude that undersized mitral annuloplasty can safely be employed in a restricted number of patients with chronic ischemic mitral regurgitation. These patients can be preoperatively recognized on the basis of echocardiographic characteristics.

Patients with symmetric tethering with anterior leaflet tethering predominance and/or enlarged and more spherical left ventricles and/or advanced diastolic dysfunction, do not benefit from undersized annuloplasty. 
Riassunto 
Gli scopi della presente tesi sono:1) Riportare i risultati a distanza (5 anni) della anuloplastica restrittiva associata a bypass aorto-coronarico in pazienti con insufficienza mitralica post-ischemica, in termini di insufficienza mitralica ricorrente, remodelling e remodelling-inverso del ventricolo sinistro, dimensioni e geometria ventricolari 2) Studiare le modificazioni a livello ventricolare, valvolare e sottovalvolare nell' ischemico cronico prima e dopo l'intervento di plastica mitralica 3) Identificare i meccanismi che determinano la ricomparsa di insufficienza mitralica 4) Identificare i fattori preoperatori che possono aessere d'aiuto per selezionare quei pazienti che possono realmente beneficiare di un anuloplastica sottodimensionata.

$\mathrm{Nel}$ Capitolo 1 abbiamo presentato i risultati ecocardiografici a 5 anni della anuloplastica restrittiva associata a bypass aortocoronarico. Duecentocinquantuno pazienti sono stato arruolati per lo studio ed un controllo ecocardiografico è stato effettuato nei 220 sopravvissuti all'intervento chirurgico. Globalmente, l'insufficienza è rimasta invariata a 1 anno mentre è aumentata significativamente ai controlli a 3 e 5 anni. La sopravvivenza attuariale a 5 anni è stata di $83.2 \pm 4.4 \%$ mentre la libertà da reintervento è stata $78.2 \pm 4.9 \%$. I diametric ventricolari hanno mostrato una significativa riduzione al controllo alla dimissione ed ai controlli precoci, con significativi aumenti a 3 e 5 anni. Gli indici di sfericità (sistolico e diastolico) si sono ridotti alla dimissione, rimanendo stabili ad 1 anno e ri-aumentando significativamente a 3 e 5 anni.L'incidenza di rimodellamento inverso è stata di $44.2 \%$ con il $10 \%$ dei pazienti che hanno mostrato un ulteriore incremento del volume telesistolico. All'analisi multivariata, un volume telesistolico $\geq 145 \mathrm{ml}$, un indice di sfericità sistolico $\geq 0.7$, un indice di performance miocardica $\geq 0.9$ ed un wall motion score Index $\geq 1.5$ sono risultati predittori di insufficienza mitralica postoperatoria. I nostri risultati enfatizzano la necessità di un'accurata selezione preoperatoria dei pazienti, che ci aiuterà a riconoscere quelli che non beneficiano di una plastica valvolare e per i quali è necessario un intervento alternativo che corregga direttamente il tethering a livello ventricolare o una sostituzione valvolare mitralica.

Nel capitolo 3 abbiamo analizzato il rimodellamento inverso dopo anuloplastica riduttiva e bypass aortocoronarico esaminando in particolare la differenza di risultato, in termini di outcome clinico e ricomparsa di insufficienza mitralica, tra responderse non-responders al rimodellamento ventricolare sinistro inverso. Duecentoquattro pazienti sopravvissuti all' intervento di anuloplastica riduttiva, sono stati oggetto dello studio. E' stato considerato rimodellamento inverso una riduzione del volume telesistolico ventricolare sinistro Indicizzato $>15 \%$. Ottantaquattro pazienti (41.2\%) hanno mostrato rimodellamento inverso mentre in $120(58.8 \%)$ non si è riscontrato una riduzione del volume telesistolico $>15 \%$. I non-responders hanno mostrato una maggiore ricorrenza di insufficienza mitralica postoperatoria, una più alta frequenza di reintervento per fallimento della procedura, un volume ventricolare e dimensioni ventricolari significativamente maggiori a tutti i controlli con un indici di sfericità che hanno raggiunto al follow up valori più alti di quello basale. L'analisi multivariata 
ha identificato come predittori indipendenti di rimodellamento inverso $\mathrm{i}$ seguenti fattori: un valore di indice di performance miocardica $<0.90$, un indice di sfericità sistolico $<0.72$ ed un wall motion score Index $<1.59$. La nostra esperienza suggerisce che un maggior numero di informazioni su i possibili predittori ecocardiografici di rimodellamento inverso possono decisamente migliorare la selezione dei pazienti in fase preoperatoria, indirizzando all'intervento quelli che realmente beneficiano di una anuloplastica riduttiva

Nel Capitolo 4 abbiamo studiato la configuazione a livello valvolare e sottovalvolare per identificare i meccanismi che determinano la ricomparsa di insufficienza mitralica ischemica dopo anuloplastica riduttiva. La popolazione dello studio è consistita in 230 pazienti divisi in due gruppi: pazienti senza insufficienza mitralica postoperatoria (Gruppo 1, $\mathrm{n}=176$ ) e pazienti con insufficienza mitralica dopo l'intervento (Gruppo 2, $n=54$ ). Cinquanta soggetti sani hanno rappresentato il Gruppo di controllo. I pazienti con insufficienza motralica postoperatoria avevano nel preoperatorio un tethering più simmetrico, un tethering del lembo anteriore più accentuato, una escursione del lembo anteriore maggiormente ridotta. Nel postoperatorio, il tethering del lembo posteriore è aumentato significativamente mentre quello del lembo anteriore si è ridotto alla dimissione, rimanendo costante successimante. La regressione logistica ha identificato un angolo di tethering del lembo anteriore $\geq 39.5^{\circ}$, un rapporto angolo di tethering anteriore / angolo di tethering posteriore $\geq 0.76$, un angolo di escursione del lembo anteriore $\leq 35^{\circ}$ ed un' altezza di coaptazione $\geq 11 \mathrm{~mm}$ come predittori indipendenti di insufficienza mitralica postoperatoria. I nostri risultati suggeriscono che la presenza di tethering simmetrico prevalentemente anteriore è una controindicazione alla anuloplastica riduttiva.

$\mathrm{Nel}$ Capitolo 5 abbiamo testato l'ipotesi che una specifica configurazione di tethering nel preoperatorio, caratterizzata da tethering simmetrico prevalentemente anteriore, è legato alla ricomparsa di insufficienza mitralica postoperatoria, indipendentemente dal volume e dalla geometria del ventricolo sinistro. Abbiamo misurato il dislocamento posteriore e laterale dei muscoli papillari, gli angoli di tethering dei lembi mitralici a gli indici di coaptazione in 278 pazienti sottoposti ad anuloplastica riduttiva per insufficienza mitralica cronica post-ischemica, ed in 50 soggetti normali. I pazienti sono stati divisi in 5 Gruppi in base all'entità del tethering del lembo anteriore: assente/lieve (Gruppo 1, $n=33$ ), medio (Gruppo 2, $n=55$ ), moderato (Gruppo 3, n=63), moderato-severo (Gruppo 4, n=64) e severo (Gruppo 5, n=63). I pazienti con tethering anteriore $\geq$ moderato-severo mostravano, all'eco preoperatorio, una maggiore dislocazione del papillare anteriore and un maggiore rimodellamento nella zona anteriore. Una insufficienza mitralica residua è stata riscontrata in percentuale significativamente maggiore in pazienti con tethering anteriore moderato-severo o severo. Dopo l'intervento, il tethering del lembo posteriore è aumentatoin tutti i Gruppi ed i pazienti con insufficienza mitralica postoperatoria mostravano un tethering prevalentemente asimmetrico. La regressione logistica ha 
202 individuato nel angolo di tethering del lembo anteriore il fattore determinante per la ricomparsa di insufficienza nel postoperatoria. Questo risultato veniva confermato anche dopo avere corretto la regressione per quei fattori determinanti la ricomparsa di insufficienza mitralica. I nostri risultati confermano che il tethering anteriore è determinante per la ricomparsa di insufficienza mitralica, indipendentemente dalla geometria e dalle dimensioni del ventricolo.

Nel Capitolo 6 abbiamo studiato l'influenza della funzione diastolica sui risultati postoperatori e sulla ricomparsa di insufficienza mitralica dopo anuloplastica riduttiva. Per questo 234 pazienti con insufficienza mitralica cronica post-ischemica sono stati divisi in 4 Gruppi sulla base del tempo di decelerazione e del rapporto tra flusso venoso polmonare sistolico e diastolico: Gruppo 1 (funzione normale, $\mathrm{n}$ $=48$ ), Gruppo 2 (alterato rilasciamento, $n=61$ ), Gruppo 3 (pattern pseudonormale, $\mathrm{n}=60$ ) e Gruppo 4 (pattern restrittivo, $\mathrm{n}=65$ ). La mortalità precoce è stata maggiore nel Gruppo con pattern restrittivo (9.2\%). Inoltre, la sopravvivenza a 6 anni è stata significativamente più bassa nel Gruppo 4. Un tempo di decelerazione $<140 \mathrm{~ms}$ ed un rapporto sisto-diastolico del flusso venoso polmonare $<0.80$ si sono rivelati fattori predittivi di morte precoce ed a distanza mentre il solo tempo di decelerazione $<140 \mathrm{~ms}$ predice la ricomparsa di insufficienza mitralica nel postoperatorio.La disfunzione diastolica, ed in particolare la presenza di un pattern restrittivo, rappresenta un indice predittivo di mortalità e di ricorrenza di insufficienza mitralica. Lo studio della funzione diastolica dovrebbe, perciò, essere effettuato regolarmente durante lo screening preoperatorio del paziente.

Nel Capitolo 7 abbiamo studiato il valore predittivo del tempo di decelerazione sul rimodellamento inverso nei pazienti con insufficienza mitralica cronica postischemica sottoposti ad anuloplastica riduttiva e bypass aorto-coronarico. Sono stati studiati 215 pazienti, divisi in $4 \mathrm{Gruppi}$ in base al valore del tempo di decelerazione: Gruppo 1 (funzione normale, $n=48$ ), Gruppo 2 (alterato rilasciamento, $n=61$ ), Gruppo 3 (pattern pseudonormale, $n=50$ ) e Gruppo 4 (pattern restrittivo, $n=$ 56). L'incidenza di rimodellamento inverso (definito come una riduzione del volume telesistolico ventricolare sinistro ESV > 15\%) nei Gruppi 1- 4 è stato 95.7\%, 96.3\%, $88.3 \%$ e $0 \%$, rispettivamente. L'analisi multivariata ha confermato che un valore di tempo di decelerazione $\leq 125$ rappresenta un fattore predittivo di rimodellamento dopo anuloplastica. Questi risultati confermano che la funzione diastolica, ed in particolare il tempo di decelerazione, sono informazioni indispensabili per una corretta valutazione preoperatoria del paziente ed una corretta indicazione chirurgica.

Possiamo concludere che l'anuloplastica riduttiva può essere utilizzata con successo in un piccolo numero di pazienti con insufficienza mitralica cronica postischemica. Un accurata valutazione preoperatoria è indispensabile ed aiuta ad identificare questi pazienti. 
I pazienti con con tethering simmetrico prevalentemente anteriore e/o con ventricolo sinistro aumentato di volume e sferico e/o con disfunzione diastolica non beneficiano di questa procedura. Per quasti pazienti dve essere valutato un intervento alternativo che corregga il tethering a livello ventricolare oppure una sostituzione valvolare mitralica. 
Samenvatting 
Ischemische mitraal klep regurgitatie (MR) is een relatief veel voorkomend verschijnsel bij coronair ziekten, en treedt op na een acuut myocard infarct (MI), of als een chronisch aandoening, en minder vaak bij plaatselijke intermitterende ischemie. Chronische ischemische mitraal klep regurgitatie (CIMR) is een functionele regurgitatie, in tegenstelling tot anatomische MR, omdat de klepbladen er normaal uitzien. CIMR heeft wel een veel slechtere prognose na een doorgemaakt myocard infarct en coronair revascularisatie (1-5), en is een veel voorkomende oorzaak van hartfalen. CMIR komt voor bij 10 tot $20 \%$ van alle patiënten met een coronair arterie aandoening (8-10).

De doelstellingen van dit proefschrift waren: 1) om de lange termijn resultaten te presenteren van een gecombineerde behandeling bestaande uit coronaire bypass chirurgie $(C A B G)$ en reductieve annuloplastie in patiënten met chronische ischemische mitraal klep regurgitatie. Als parameters zijn gekozen de terugkeer van mitraal klep regurgitatie, linker ventrikel reverse remodeling, en meer algemeen de linker ventrikel dimensies en geometrie. 2) om de veranderingen te beschrijven in de bouw en de vorm van de linker ventrikel en mitraal klep die optreden na annuloplastie, bij patiënten met CIMR. 3) om die mechanismen te herkennen die betrokken zijn bij het terugkomen van mitraal klep regurgitatie, zij het ventriculair of valvulair. 4) om preoperatief eigenschappen te herkennen zodat patiënten beter kunnen worden geselecteerd die potentieel voordeel hebben bij annuloplastie.

In hoofdstuk 2 worden de resultaten gepresenteerd van het echo-onderzoek 5 jaar na een gecombineerde CABG en mitraal klep annuloplastie, in patiënten met CIMR. 251 Patiënten werden prospectief in de studie opgenomen. In 220 overlevende patiënten werd een echocardiogram gemaakt voor de operatie, bij ziekenhuis ontslag, en daarna jaarlijks.

MR bleef stabiel na het eerste jaar, maar nam weer toe na het derde en vijfde jaar. De overleving na 5-jaar was $83,2 \pm 4,4 \%$. De 5-jaars vrijheid van reoperatie doordat de hersteloperatie onvoldoende resultaat opleverde was $78,2 \pm 4,9 \%$. De gemiddelde LV systolische en diastolische diameter waren bij ontslag uit het ziekenhuis en na het eerste jaar significant afgenomen, maar namen weer toe na 3 en na 5 jaar. De systolische en diastolische index voor de LV bolvorm was verbeterd bij ontslag, bleef vervolgens het eerste jaar stabiel, maar nam weer toe bij de controle na 3 jaar, en later zelfs meer wat tot een grotere index leidde dan voor de ingreep. Linker ventrikel reverse remodeling werd waargenomen bij $44,2 \%$ van de studie groep, terwijl in $10,3 \%$ een verdere dilatatie werd gemeten. Uit multivariate logistische analyse bleken verschillende factoren de terugkeer van MR te kunnen voorspellen: Eind systolisch volume $\geq 145 \mathrm{ml}, \mathrm{LV}$ systolische bolindex $\geq 0,7$, Hart functie index $\geq 0,9$ en index voor de wall motion score $\geq 1,5$.

De uitkomsten van deze studie benadrukken de noodzaak van een betere chirurgische herstel techniek van de mitraal klep, en een betere patiënten selectie, om patiënten te kunnen herkennen met een voorspelbare klepherstel ingreep zonder 
resultaat, die meer baat zouden kunnen hebben bij klepvervanging of een andere procedure waardoor de ventriculaire aanhechtingen worden verbeterd.

In hoofdstuk 3 is linker ventrikel reverse remodeling bestudeerd na een gecombineerde ingreep die bestond uit $C A B G$, en een onderbemeten mitraalklep annuloplasty. Hierbij is gekeken naar het verschil in verbetering door de annuloplastie op klinische eindpunten en op terugkeer van MR, tussen patiënten die wel of juist niet linker ventrikel reverse remodeling lieten zien. Voor deze studie kwamen 204 patiënten in aanmerking, die de gecombineerde ingreep overleefden van de CABG en de reductieve annuloplastie. Reverse remodeling was gedefinieerd als een vermindering in LV eind-systole volume index $>15 \%$. Er waren $84(41,2 \%)$ responders van reverse remodeling, en $120(58,8 \%)$ non-responders. Bij deze non-responders kwam terugkeer van MR meer voor, was er een grotere mate van reoperaties en significant grotere LV dimensies en volumina op ieder tijdstip, met significante late toename van de bolvorm index, tot zelfs groter dan de waarde voor de chirurgie. Bij multivariate analyse bleken vooraf gemeten hart functie index $<0,90$, systolische bolvorm index $<0,72$, en wall motion score $<1,59$ onafhankelijke voorspellers van reverse remodeling.

Onze ervaring suggereert dat echo data als mogelijke voorspellers van eindresultaat, de preoperatieve patiënten selectie en de keuze van het type ingreep zou kunnen verbeteren.

In hoofdstuk 4 wordt een onderzoek beschreven naar de mitraal klep bladen en subvalvulaire structuren, om mechanismen te herkennen die leiden tot het terugkeren van $M R$, in de fase na een $C A B G$ en mitraal annuloplastie.

De onderzoeks groep bestond uit 230 patiënten die in twee groepen werden verdeeld: Groep 1 ( $n=176)$ zonder late MR, en Groep $2(n=54)$ met late terugkerende $\mathrm{MR}$. Een groep van 50 gezonde vrijwilligers werd genomen als controle.

Patiënten in Groep 2 bleken preoperatief een meer symmetrische tethering te hebben, een meer benadrukte anterior mitraal klep tethering, en een meer beperkte anterior klep blad excursie, in vergelijking met Groep 1. Postoperatief nam de tethering van het posterior klepblad toe, zowel in groep 1 als in groep 2, terwijl de tethering van het anterior klepblad verminderd was bij ontslag, en daarna onveranderd bleef. Multivariate analyse liet zien welke variabelen voorspellers waren van het terugkomen van MR: anterior tethering hoek $>39,5^{\circ}$, een anterior/posterior tethering hoek verhouding $>0,76$, een anterior klepblad excursie hoek $<35^{\circ}$, en een coaptatie hoogte $>11 \mathrm{~mm}$.

Onze resultaten laten zien dat preoperatieve symmetrische tethering met overheersend anterior mitraal klepblad sterk is geassocieerd met het terug keren van MI.

In hoofdstuk 5 wordt de hypothese getoetst die stelt dat een specifieke vorm van het klepblad met een versterkte anterior klepblad tethering, is gerelateerd aan een hogere mate van postoperatieve terugkeer van MR, onafhankelijk van de linker 
ventrikel grootte en vorm. In 278 patiënten met chirurgische annuloplastie voor de behandeling van CIMR, en in 50 gematchte normale personen, werden voor de ingreep en tijdens de follow up verschillende variabelen bestudeerd; posterior en laterale verplaatsing van de papilair spieren, de tethering hoek en hun ratio van de anterior en de posterior klep bladen. De patiënten werden verdeeld in 5 groepen op basis van de hoek van tethering van het anterior mitraal klepblad voor de operatie. Groep 1 (normale/geringe tethering, $n=33$ ), groep 2 (matige tethering, $n=55$ ), groep 3 ( middelmatige tethering, $\mathrm{n}=63$ ), groep 4 (middelmatige tot ernstige tethering, $\mathrm{n}=64$ ) en groep 5 (ernstige tethering, $\mathrm{n}=63$ ). Het bleek dat patiënten met een tethering van het anterior mitraal klepblad van meer dan middelmatig, preoperatief een meer symmetrische tethering hadden, een meer versterkte anterior papilair spierverplaatsing en een grotere remodeling van de linker ventrikal voorwand. Terugkeer van MR was significant vaker in patiënten met middelmatige tot ernstige anterior mitraal klepblad tethering.

Postoperatief nam de tethering van het posterior klepblad toe, maar de mate waarin verschilde niet tussen de groepen. Het resulteerde in een meer symmetrische tethering bij de meeste patiënten met terugkerende MR. Multivariate logistische regressie toonde aan dat de tethering hoek van het anterior klepblad de primaire voorspeller was van terugkerende $M R$, zelfs na aanpassen van bekende risicofactoren. Onze resultaten bevestigen dat de mate van preoperatief anterior mitraal klepblad tethering sterk is geassocieerd met de terugkeer van ischemische MR.

In hoofdstuk 6 wordt de voorspellende waarde beschreven van diastolische hartfunctie op de klinische outcome en op het terugkeren van ischemische MR, bij 234 patiënten die behandeld zijn met een onderbemeten mitraal annuloplastie en een CABG. De patiënten werden verdeeld in 4 groepen op basis van deceleration time en de systolische/diastolische longvene flowratio: Groep 1, normaal $(n=48)$, Groep 2, verminderde relaxatie ( $n=61)$, Groep 3, pseudonormaal patroon $(n=60)$, en Groep 4, restrictief patroon $(n=65)$. De sterfte direct na de ingreep was het hoogst in groep 4 (9.2\%), de groep met een restrictief patroon. Daarnaast bleek ook de 6 jaars overleving lager in groep 4. Ook bleek dat een deceleratie tijd van $<140 \mathrm{~ms}$, en een systolisch/diastolisch longvene flow ratio van $<0.80$, onafhankelijke voorspellers waren van het terugkeren van ischemische MR. Een restrictief diastolisch vullings patroon van de linker ventrikel is een belangrijke preoperatieve marker voor een hoge vroege en late mortaliteit.

In hoofdstuk 7 wordt de voorspellende waarde besproken die de deceleratietijd van de mitraal klep heeft op de reverse remodeling van de linker ventrikel, in patiënten met CIMR, na een chirurgische behandeling. Deze behandeling bestond uit $C A B G$ gecombineerd met undersized mitraal annuloplastie, en werd uitgevoerd in 215 patiënten. Deze patiënten werden in 4 groepen ingedeeld, analoog als in hoofdstuk 6. De linker ventrikel reverse remodeling werd gedefinieerd als een vermindering van de LV eind systolisch volume index $>15 \%$, en bleek sterk 
te verschillen tussen de groepen (Groep 1:95,7\%, Groep $2: 96,3 \%$, Groep 3 : $88,3 \%$, Groep $4: 0 \%$ ). Logistische regressie analyse liet zien dat een decelleratie tijd van $\leq 125 \mathrm{~ms}$ een sterke voorspeller was voor reverse remodeling na annuloplastie. Preoperatieve bepaling van deze parameter voegt belangrijke informatie toe aan de gebruikelijke indexes van globale en regionale functie, en vormen een eenvoudige en kosteneffectieve manier om nauwkeurig die patiënten te herkennen die werkelijk baat kunnen hebben bij een annuloplastie.

Als conclusie kan worden gesteld, dat undersized mitraal annuloplastie veilig kan worden uitgevoerd in een beperkt aantal patiënten met CIMR. Deze patiënten kunnen preoperatief worden herkend op basis van echocardiografisch verkregen gegevens. Bij patiënten die geen baat zullen hebben van een undersized annuloplastie, wordt het meest frequent symmetrische tethering gezien, met voornamelijk anterior klepblad tethering en/of een vergroot en meer bolvormige linker ventrikel, en/of vergevorderde diastolische disfunctie. 
Publications 
1. Lorusso R, Corradi D, Maestri R, Bosio S, Curulli A, Beghi C, Gerometta P, Russo C, Gelsomino S, Moreo A, De Cicco G, Rosano G, Volterrani M. Atorvastatin attenuates post-implant tissue degeneration of cardiac prosthetic valve bovine pericardial tissue in a subcutaneous animal model. Int J Cardiol. 2009 Jan 21. [Epub ahead of print]

2. Gelsomino S, Lorusso R, De Cicco G, Billè G, Caciolli S, Rostagno C, Capecchi I, Chioccioli M, Stefàno P, Gensini GF. Does preoperative tethering symmetry affect left ventricular reverse remodeling after restrictive annuloplasty? Int J Cardiol. 2009 Jan 19. [Epub ahead of print]

3. Bevilacqua S, Romagnoli S, Ciappi F, Lazzeri C, Gelsomino S, Pulli R, Pratesi C, Gensini GF. Patient Cooperation During General Anesthesia for Combined Carotid and Coronary Artery Surgery. J Cardiothorac Vasc Anesth. 2008 Oct 2. [Epub ahead of print]

4. Bevilacqua S, Lazzeri C, Romagnoli S, Gelsomino S, Lorusso R, De Cicco G, Gensini GF. Unexpected Severe Tricuspid Regurgitation After Successful Mitral Valve Repair.J Cardiothorac Vasc Anesth. 2008 Apr 15. [Epub ahead of print]

5. Bonacchi M, Nistri S, Nanni C, Gelsomino S, Pini A, Cinci L, Maiani M, Zecchi Orlandini S, Lo russo R, Fanti L, Silvertown J, Bani D. Functional and histopathological improvement of the post-infarcted rat heart upon myoblast cell grafting and relaxin therapy. Journal of Cellular and Molecular Medicine 288 (Epub Ahead of Print).

6. Caciolli S, Gelsomino S, Fradella G, Bevilacqua S, Favilli S, Gensini GF. Severe Hypoplasia of the Posterior Mitral Leaflet. Ann Thorac Surg 2008; 86 (6): 1978-9.

7. Lorusso R, De Cicco G, Tironi A, Gelsomino S, De Geest R. Giant Primary Paraganglioma of The Left Ventricle. J Thorac Cardiovasc Surg. 2009; 137 (2): 499-500.

8. Lorusso R, Gelsomino S. El Corazon Espinado. The Lancet "in press".

9. Lorusso R, De Cicco G, Ettori F, Curello S, Gelsomino S, Fucci C.Emergency surgery after saphenous vein graft perforation complicated by catheter balloon entrapment and hemorrhagic shock. Ann Thorac Surg 2008; 86 (3): 2002-4. 
10. Lorusso R, De Cicco G, Gelsomino S. Reply to Foroughi et al. Eur J Cardiothorac Surg. 2008 Aug 11. [Epub ahead of print].

11. Gelsomino S, Lorusso R, Caciolli S, Capecchi I, Rostagno C, Chioccioli M, De Cicco G, Billè G, Stefàno P, Gensini GF. Insights on left ventricular and valvular mechanisms of recurrent ischemic mitral regurgitation after restrictive annuloplasty and coronary artery bypass grafting. J Thorac Cardiovasc Surg. 2008;136(2):507-18.

12. Lorusso R, De Cicco G, Totaro P, Gelsomino S. Effects of phosphorylcholine coating on extracorporeal circulation management and postoperative outcome: a double-blind randomized study. Interact Cardiovasc Thorac Surg. 2009; $8(1): 7-11$.

13. Gelsomino S, Lorusso R, Rostagno C, Caciolli S, Billè G, De Cicco G, Romagnoli S, Porciani C, Stefàno P, Gensini GF. Prognostic value of Doppler-derived mitral deceleration time on left ventricular reverse remodelling after undersized mitral annuloplasty. Eur J Echocardiogr. 2008 Mar 11.

14. Gelsomino S, Lorusso R, Billè G, Rostagno C, De Cicco G, Romagnoli S, Porciani C, Tetta C, Stefàno P, Gensini GF. Left ventricular diastolic function after restrictive mitral ring annuloplasty in chronic ischemic mitral regurgitation and its predictive value on outcome and recurrence of regurgitation. Int J Cardiol. 2008 Mar 26.

15. Gelsomino S, Lorusso R, Capecchi I, Rostagno C, Romagnoli S, Billè G, De Cicco G, Tetta C, Stefàno P, Gensini GF. Left ventricular reverse remodeling after undersized mitral ring annuloplasty in patients with ischemic regurgitation. Ann Thorac Surg. 2008 Apr;85(4):1319-30.

16. Lorusso R, Gelsomino S, De Cicco G, Beghi C, Russo C, De Bonis M, Colli A, Sala A. Mitral valve surgery in emergency for severe acute regurgitation: analysis of postoperative results from a multicentre study. Eur J Cardiothorac Surg. 2008 Apr;33(4):573-82.

17. Gelsomino S, Lorusso R, Billè G, De Cicco G, Da Broi U, Rostagno C, Stefàno $P$, Gensini GF. Cardiac surgery in type-1-myotonic muscular dystrophy (Steinert syndrome) associated to Barlow disease.Interact Cardiovasc Thorac Surg. 2008 Apr;7(2):222-6. 
18. Gelsomino S, Lorusso R, Romagnoli S, Bevilacqua S, De Cicco G, Billè G, Stefàno $\mathrm{P}$, Gensini GF.Treatment of refractory bleeding after cardiac operations with low-dose recombinant activated factor VII (NovoSeven): a propensity score analysis.Eur J Cardiothorac Surg. 2008 Jan;33(1):64-71.

19. Gelsomino S, Lorusso R, De Cicco G, Capecchi I, Rostagno C, Caciolli S, Romagnoli S, Da Broi U, Stefàno P, Gensini GF. Five-year echocardiographic results of combined undersized mitral ring annuloplasty and coronary artery bypass grafting for chronic ischaemic mitral regurgitation. Eur Heart J. 2008 Jan;29(2):231-40.

20. Lorusso R, De Cicco G, Gelsomino S, Fucci C. Dissection of the posterior mitral leaflet. Eur J Cardiothorac Surg. 2007 Dec;32(6):936.

21. Lorusso R, Gelsomino S, De Cicco G. Acute pulmonary embolism after removal of central venous catheter. Int J Cardiol. 2007 Sep 18.

22. Pavoni D, Badano LP, Ius F, Mazzaro E, Frassani R, Gelsomino S, Livi U. Limited long-term durability of the Cryolife O'Brien stentless porcine xenograft valve. Circulation. 2007 Sep 11;116(11 Suppl):I307-13 .

23. Braconi L, Pretelli P, Calamai G, Montesi G, Romagnoli S, Gelsomino S, Bevilacqua $S$, Stefàno P, Gensini GF. An unusual case of cardiac tamponade. J Cardiovasc Med (Hagerstown). 2006 Mar;7(3):219-21.

24. Romagnoli S, Bevilacqua S, Gelsomino S, Pradella S, Ghilli L, Rostagno C, Gensini GF, Sorbara C. Small-dose recombinant activated factor VII (NovoSeven) in cardiac surgery. Anesth Analg. 2006 May;102(5):1320-6.

25. Bevilacqua $S$, Gelsomino $S$, Romagnoli $S$. Difficult intubation in pediatric cardiac surgery. J Cardiothorac Vasc Anesth. 2006 Apr;20(2):290-1.

26. Gelsomino S, Romagnoli S, Gori F, Nesi G, Anichini C, Sorbara C, Stefàno P, Gensini GF. Annuloaortic ectasia and giant cell arteritis. Ann Thorac Surg. $2005 \mathrm{Jul} ; 80(1): 101-5$.

27. Gelsomino S, Romagnoli S, Dragotto A, Cassai M, Fiorani B, Sorbara C, Stefàno P. Multistep endobronchial-endovascular approach in recurrent acute respiratory failure caused by thoracic aneurysm.J Thorac Cardiovasc Surg. 2005 Jun;129(6):1436-8. 
28. Gelsomino S, Romagnoli S, Stefàno P. Right coronary perforation due to a toothpick ingested at a barbecue. N Engl J Med. 2005 May 26;352(21):224950.

29. Gelsomino S, Morocutti G, Masullo G, Da Col P, Frassani R, Spedicato L, Livi U. Patient-prosthesis mismatch after small-size stentless aortic valve replacement. J Card Surg. 2004 Mar-Apr;19(2):91-7.

30. Gelsomino S, Rostagno C, Bevilacqua S, Caciolli S, Capecchi I, Casolo G, Gensini GF, Stefano P. A complex triple valve repair in a young rheumatic patient.Ital Heart J. 2003 Dec;4(12):884-7.

31. Gelsomino S, Masullo G, Morocutti G, Frassani R, Da Col P, Parise O, Spedicato L, Livi U. Sixteen-year results of composite aortic root replacement for non-dissecting chronic aortic aneurysms. Ital Heart J. 2003 Jul;4(7):454-9.

32. Gelsomino S, Morocutti G, Frassani R, Masullo G, Da Col P, Spedicato L, Livi U. Long-term results of Bentall composite aortic root replacement for ascending aortic aneurysms and dissections. Chest. 2003 Sep;124(3):984-8.

33. Gelsomino S, Rubattu G, Terrosu PF, Cossu L, Orrù F, Barboso G. Successful repair of a coronary artery to pulmonary artery fistula with saccular artery aneurysm and critical stenosis of the left anterior descending coronary artery. Ital Heart J. 2003 May;4(5):350-3.

34. Gelsomino S, Morocutti G, Da Col P, Masullo G, Carella R, Guzzi G, Spedicato $L$, Livi $U$. Early in vivo hemodynamic results after aortic valve replacement with the St Jude Medical Regent mechanical heart valve in patients with pure aortic stenosis. J Card Surg. 2003 Mar-Apr;18(2):125-32.

35. Morocutti G, Gelsomino S, Spedicato L, Shiety Toh H, Pitzorno C, Masullo G, Bernardi G, Da Col P, Frassani R. Transesophageal echocardiography follow-up of patients undergoing replacement of the ascending aorta and aortic valve with a Cabrol procedure for chronic aneurysm and dissection. J Am Soc Echocardiogr. 2003 Apr;16(4):360-6.

36. Gelsomino S, Frassani R, Morocutti G, Masullo G, Da Col P, Spedicato L, Porreca L, Livi U. A short-term experience with the Tirone David I valve sparing operation for the treatment of aneurysms of the ascending aorta and aortic root. Cardiovasc Surg. 2003 Jun;11(3):189-94. 
37. Gelsomino S, Frassani R, Da Col P, Morocutti G, Masullo G, Spedicato L, Livi $U$. A long-term experience with the Cabrol root replacement technique for the management of ascending aortic aneurysms and dissections. Ann Thorac Surg. $2003 \operatorname{Jan} ; 75(1): 126-31$.

38. Morocutti G, Spedicato L, Gelsomino S, Toh HS, Bernardi G, Fioretti P. Use of barcodes of biomedical products in a catheterization laboratory. A required step to improve quality and reduce costs. Ital Heart J Suppl. 2002 Aug;3(8):851-7.

39. Morocutti G, Gelsomino S, Spedicato L, Frassani R, Bernardi G, Da Col P, Paparella G, Fioretti P, Livi U. Intraoperative transesophageal echo-Doppler evaluation of stentless aortic xenografts. Incidence and significance of moderate gradients. Cardiovasc Surg. 2002 Aug;10(4):328-32.

40. Morocutti G, Gasparini D, Spedicato L, Gelsomino S, Paparella G, Bernardi $G$, Fioretti P. Functional evaluation of steal by unligated first intercostal branch before transcatheter embolization in recurrent angina after a LIMA-LAD graft. Catheter Cardiovasc Interv. 2002 Jul;56(3):373-6.

41. Gelsomino S, Morocutti G, Da Col P, Frassani R, Carella R, Minen G, Livi $U$. Preliminary experience with the St. Jude Medical Regent mechanical heart valve in the aortic position: early in vivo hemodynamic results. Ann Thorac Surg. 2002 Jun;73(6):1830-6;

42. Morocutti G, Bernardi G, Gelsomino S. Prosthetic valve dysfunction presenting as intermittent acute aortic regurgitation. Heart. 2002 Jul;88(1):3.

43. Gelsomino S, Morelli A, Porreca L, Morocutti G, Da Col P, Livi U. Videoassisted thoracoscopic surgery for primary chylopericardium after orthotopic heart transplantation. J Heart Lung Transplant. 2002 Jun;21(6):701-5.

44. Gelsomino S, Da Col P, Morocutti G, Frassani R, Porreca L, Minen G, Livi U. Stentless aortic valve replacement in elderly patients. Cardiovasc Surg. 2002 Jun;10(3):233-7.

45. Gelsomino S, Morocutti G, Frassani R, Da Col P, Carella R, Livi U. Usefulness of the Cryolife O'Brien stentless suprannular aortic valve to prevent prosthesis-patient mismatch in the small aortic root. J Am Coll Cardiol. 2002 Jun 5;39(11):1845-51. 
46. Morocutti G, Gelsomino S, Bernardi G. Covered stent for iatrogenic coronary arteriovenous fistula in heart transplant recipient. Heart. 2002 Apr;87(4):339.

47. Gelsomino S, Morocutti G, Masullo G, Cheli G, Poldini F, Da Broi U, Livi U. Open heart surgery in patients with dialysis-dependent renal insufficiency. J Card Surg. 2001 Sep-Oct;16(5):400-7.

48. Gelsomino S, Morocutti G, Frassani R, Da Col P, Morelli A, Spedicato L, Minen $G$, Livi U. Early recovery of left ventricular function after stentless versus stented aortic valve replacement for pure aortic stenosis and severe cardiac dysfunction. Semin Thorac Cardiovasc Surg. 2001 Oct;13(4 Suppl 1):120-8.

49. Gelsomino S, Frassani R, Morocutti G, Porreca L, Tursi V, Masullo G, Lumini $R$, Livi $U$. Left ventricular mass regression after aortic valve replacement with CryoLife-O'Brien stentless aortic bioprosthesis. J Heart Valve Dis. 2001 Sep;10(5):603-10.

50. Spedicato L, Bonin M, Bernardi G, Gelsomino S, Fioretti PM, Morocutti G. "Side balloon stenting": a novel technique for bifurcation lesions. J Invasive Cardiol. 2001 Oct;13(10):684-8.

51. Gelsomino S, Frassani R, Morocutti G, Nucifora R, Da Col P, Minen G, Morelli A, Livi $U$. Time course of left ventricular remodeling after stentless aortic valve replacement. Am Heart J. 2001 Sep;142(3):556-62.PMID: 11526373 [PubMed - indexed for MEDLINE]

52. Livi U, Gelsomino S, Da Col P, Poldini F, Masullo G, Cheli G, Nucifora R, Muzzi R, Da Broi U. The A-Med right heart support for off-pump coronary artery bypass grafting. Ital Heart J. 2001 Jul;2(7):502-6.

53. Gelsomino S, Da Col P, Frassani R, Morocutti G, Muzzi R, Bassi F, Cappelletto $\mathrm{G}$, Livi U. Coronary artery bypass grafting in patients with dialysis-dependent renal failure: ten-year results. Ital Heart J. 2001 May;2(5):379-83.

54. Gelsomino S, Frassani R, Porreca L, Morocutti G, Morelli A, Livi U. Early and midterm results of model 300 CryoLife O'Brien stentless porcine aortic bioprosthesis. Ann Thorac Surg. 2001 May;71(5 Suppl):S297-301.

55. Gelsomino S, Frassani R, Porreca L, Livi U. Aortic repair in Marfan's syndrome: prevention of mechanical leaflets damage. Cardiovasc Surg. 2001 Jun;9(3):299301. 
56. Gelsomino S, Frassani R, DaCol P, Porreca L, Masullo G, Morocutti G, Livi U. The CryoLife O'Brien stentless porcine aortic bioprosthesis: 5-year follow-up. Ann Thorac Surg. 2001 Jan;71(1):86-91.

57. Gelsomino S, Frassani R, Porreca L, Morocutti G, Livi U. Clinical and hemodynamic evaluation of $21 \mathrm{~mm}$ and $23 \mathrm{~mm}$ Cryolife-O'Brien stentless bioprostheses implanted in the aortic position.Ital Heart J. 2000 Sep;1(9):613-20.

58. Frassani R, Gelsomino S. A right atrial approach in redo postinfarction ventricular septal defect.Cardiovasc Surg. 1999 Oct;7(6):656-8.

59. Frassani R, Gelsomino S, Puricelli C. The recurrence of a postinfarct interventricular defect. An alternative approach G Ital Cardiol. 1998 Dec;28(12):140912.

60. Frassani R, Gelsomino S, Puricelli C. The Tirone David operation in a young patient with Marfan syndrome. Description of a clinical case.G Ital Cardiol. 1998 Sep;28(9):1017-20. 
Curriculum Vitae 
s. Born in Cosenza, Italy, on the 24/07/1962.

5. Graduated at The University of Bologna (magna cum laude), Italy.

2. Cardiac Surgery Degree at The University of Bologna (magna cum laude), Italy.

2. Recipient of Award of University of Bologna, Bologna, Italy, 1990.

* Senior House Officer (SHO), Bristol Royal Infirmary, Bristol, UK.

*. Senior House Officer, County Hospital, Hereford, UK.

2. Clinical Fellow, Cardiac Surgery, Brescia, Italy.

2. Surgical Assistant, Cardiac Surgery, Torino, Italy.

* Senior Cardiac Surgeon, Cardiac Surgery, Udine, Italy.

tw Senior Cardiac Surgeon, Cardiac Surgery, Sassari, Italy.

* Senior Cardiac Surgeon, Cardiac Surgery, Florence, Italy.

* Chief Experimental Cardiac Surgery, Florence, Italy.

\section{MEMBERSHIPS}

Society of Cardiovascular Research

S* Italian Society of Cardiac Surgery

Society of Heart Valve Disease 